\title{
An Evaluation of Corporate Social Responsibility (CSR) in Small and Medium-Sized Enterprises (SMEs): The Role of Moral Perspectives
}

\author{
Davies, Fern B.
}

How to cite:

Davies, Fern B. (2018) An Evaluation of Corporate Social Responsibility (CSR) in Small and Medium-Sized Enterprises (SMEs): The Role of Moral Perspectives. Doctoral thesis, Swansea University.

http://cronfa.swan.ac.uk/Record/cronfa48317

Use policy:

This item is brought to you by Swansea University. Any person downloading material is agreeing to abide by the terms of the repository licence: copies of full text items may be used or reproduced in any format or medium, without prior permission for personal research or study, educational or non-commercial purposes only. The copyright for any work remains with the original author unless otherwise specified. The full-text must not be sold in any format or medium without the formal permission of the copyright holder. Permission for multiple reproductions should be obtained from the original author.

Authors are personally responsible for adhering to copyright and publisher restrictions when uploading content to the repository.

Please link to the metadata record in the Swansea University repository, Cronfa (link given in the citation reference above.)

http://www.swansea.ac.uk/library/researchsupport/ris-support/ 


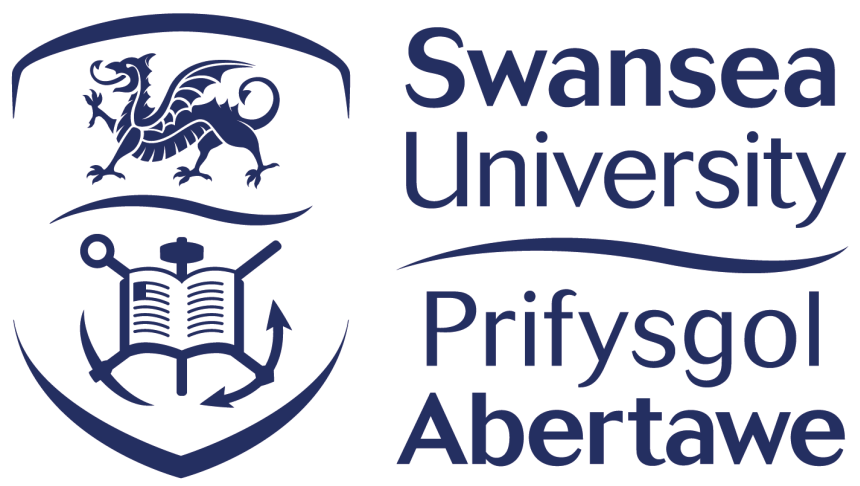

An Evaluation of Corporate Social Responsibility (CSR) in Small and Medium-Sized Enterprises (SMEs): The Role of Moral Perspectives

Fern Briony Davies B.Sc.

ORCHiD: 0000-0002-9681-912X

Submitted to Swansea University in fulfilment of the requirements for the Degree of Doctor of Philosophy

Swansea University 


\begin{abstract}
This research evaluates how corporate social responsibility (CSR) is constructed and practised in small and medium-sized enterprises (SMEs). The majority of extant research has been in large businesses, but an expanding body of CSR literature does acknowledge the distinct characteristics of SMEs, identifying ways to advance an appropriate research agenda. A significant strand of this literature calls to reclaim the moral foundations of CSR, following recognition that the concept has been constricted by economically rational justifications and a search for the business case. Consequently, a need is identified for practical and theoretical progressions that more accurately explain CSR in SMEs and address the subordination of morality.
\end{abstract}

The following study responds to the calls above and aims to establish the role of two moral perspectives: moral proximity and the ethic of care. Their relevance is explored through an empirical analysis of Spence's (2016) redrawn stakeholder theory and small business social responsibility (SBSR) pyramids. In order to do this, the research adopts a social constructionist perspective, drawing from the experiences of owner-managers through four exploratory focus groups and thirty indepth interviews. To access a deeper comprehension of CSR, the research is not only framed by ethical theory, but builds on insights from the sociology of economic behaviour.

A qualitative content analysis indicates that SMEs rarely justify their CSR engagement in rational economic terms. Motivations go far beyond the economic, with ethical and organisational perspectives most commonly represented. The research confirms that moral proximity and the ethic of care are relevant to accommodate the contextual, relational and dynamic nature of SMEs. These characteristics intensify ethical responsibility towards stakeholders, with both positive and negative implications that regardless, significantly shape the inception and engagement of CSR. From the findings, suggestions are made to enhance the redrawn theories and reiterate the value of these moral perspectives to inform our understanding of CSR in SMEs. 


\section{DECLARATION}

This work has not previously been accepted in substance for any degree and is not being concurrently submitted in candidature for any degree.

Signed. (candidate)

Date.

\section{STATEMENT 1}

This thesis is the result of my own independent investigations, except where otherwise stated.

Other sources are acknowledged by footnotes giving explicit references. A list of references is appended.

Signed.......................................... (candidate)

Date.

\section{STATEMENT 2}

I hereby give my consent for my thesis, if accepted, to be available for photocopying and for inter-library loan, and for the title and summary to be made available to outside organisations.

Signed. (candidate)

Date 


\section{Table of Contents}

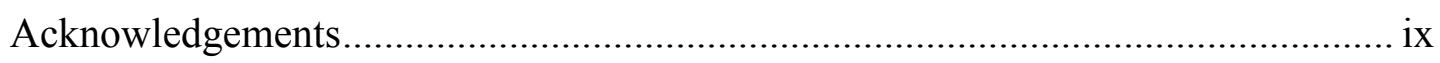

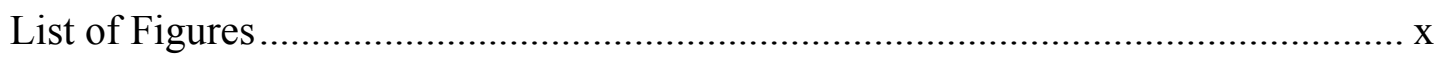

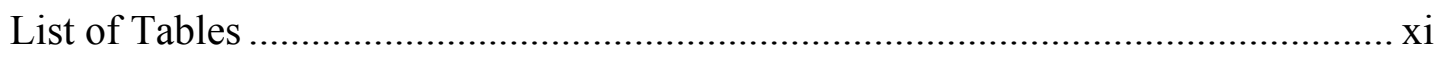

List of Appendices ..................................................................................... xii

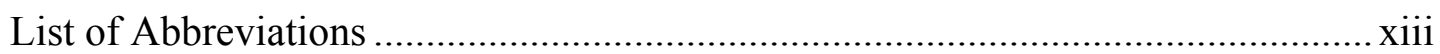

Publications from this Research ....................................................................... xiv

Chapter One: Introduction ................................................................................... 1

1.1: Research Background ............................................................................. 1

1.2: Theoretical Framing.................................................................................. 4

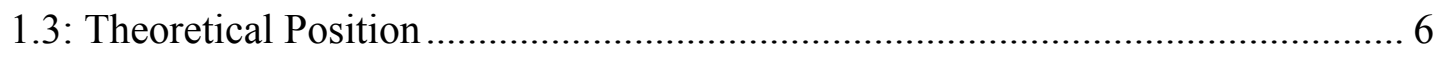

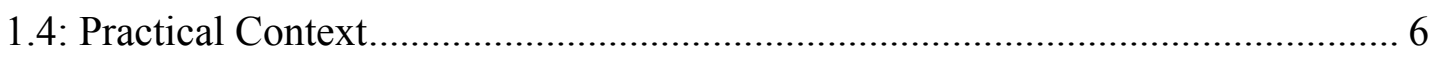

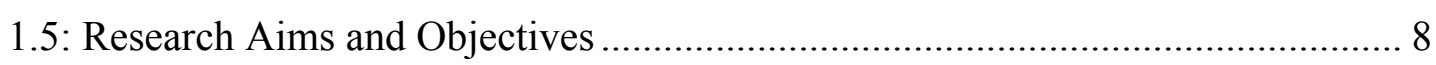

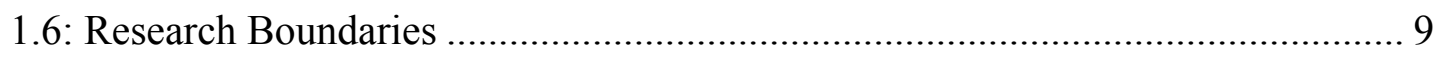

1.7: Research Contributions............................................................................. 10

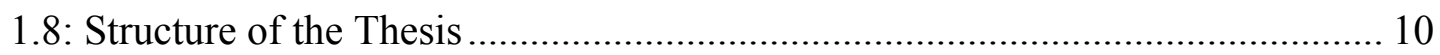

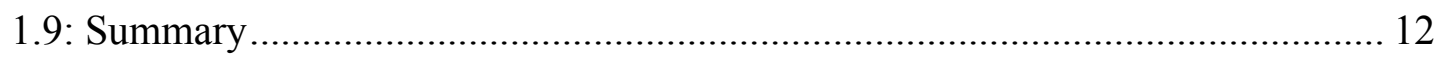

Chapter Two: Literature Review - Theoretical Foundations of CSR ……………..... 15

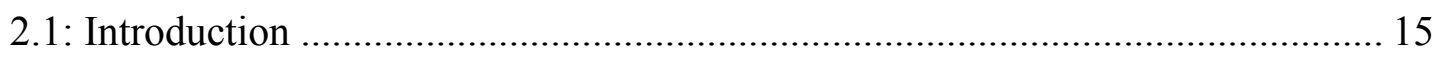

2.2: Sociological Theories ………………………………………………. 15

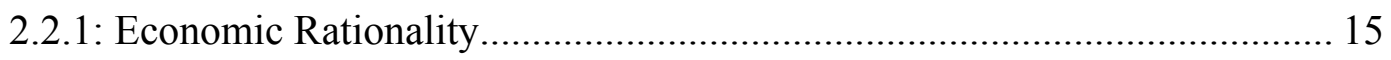

2.2.2: Bureaucratisation and the Exclusion of Feeling ....................................... 18

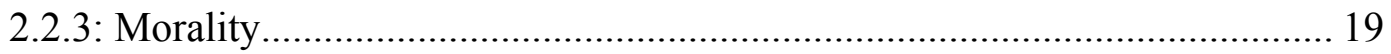

2.2.4: Embeddedness and Reciprocation ........................................................ 21

2.2.5: The Development of Moral Philosophy .................................................... 22

2.2.6: Moral Standards of Society .................................................................. 24

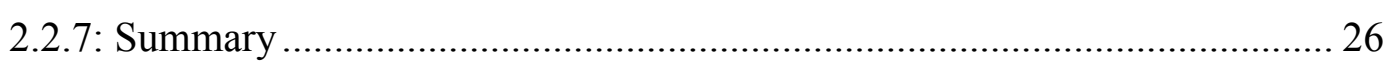

2.3: Ethical Theories ……………………………………………………. 27

2.3.1: Utilitarianism .............................................................................. 29

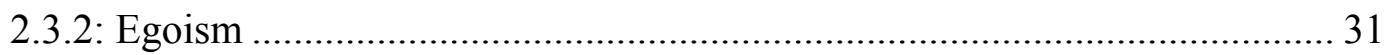




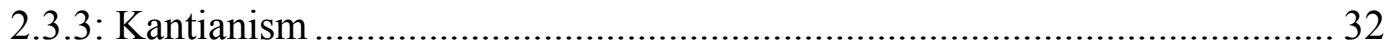

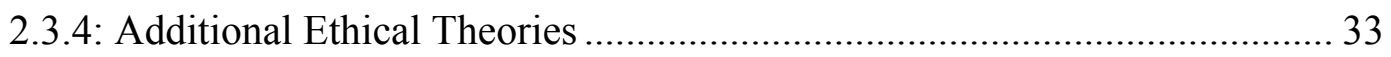

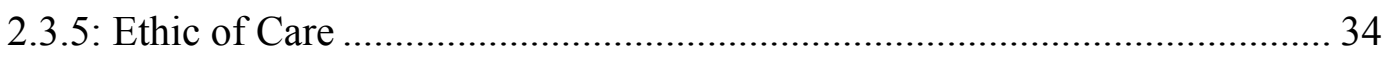

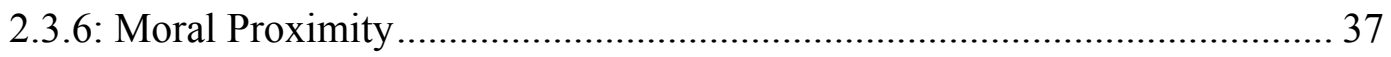

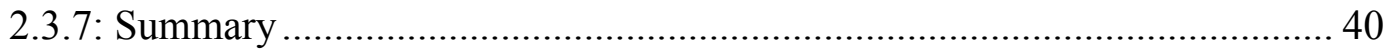

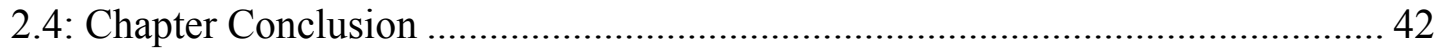

Chapter Three: Literature Review - Corporate Social Responsibility ...................... 44

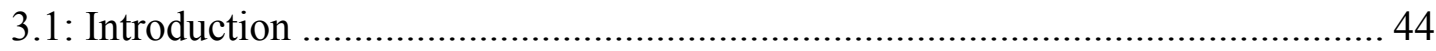

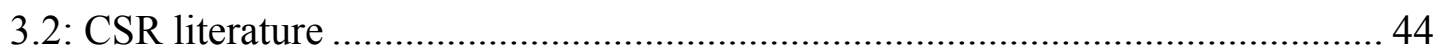

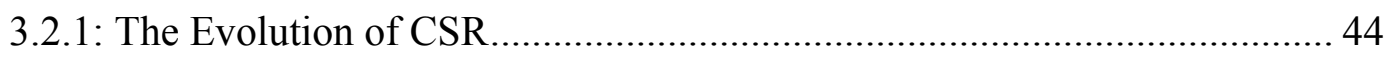

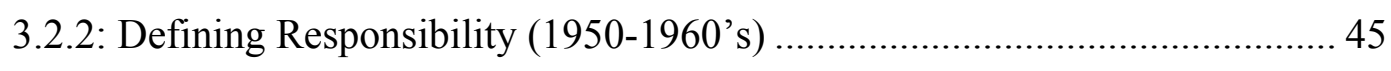

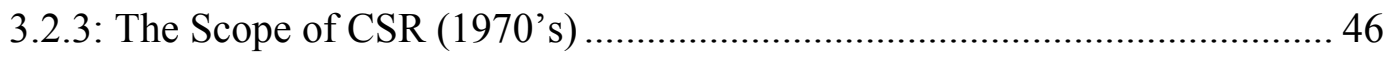

3.2.4: CSR Boundaries and Implications (1980's) .............................................. 49

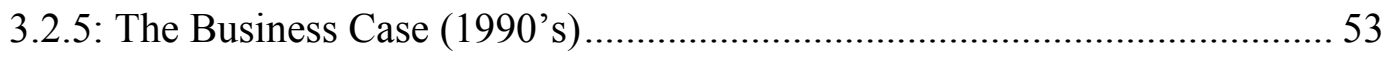

3.2.6: Strategic Orientation and Empirical Exploration (21st century) ................ 56

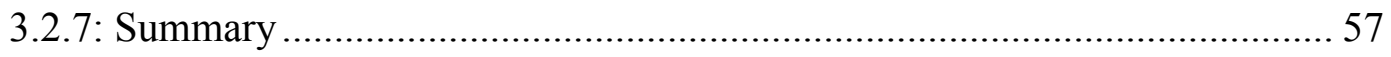

3.3: Corporate Social Responsibility (CSR) in Small and Medium-sized Enterprises

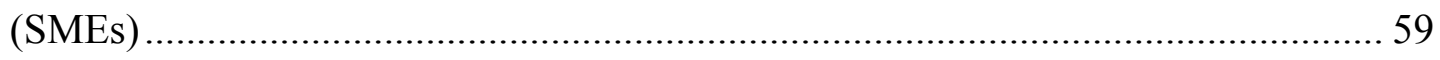

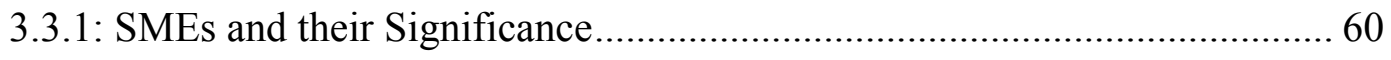

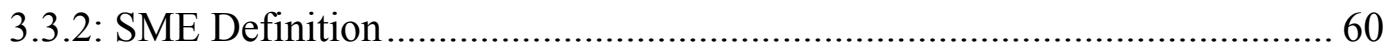

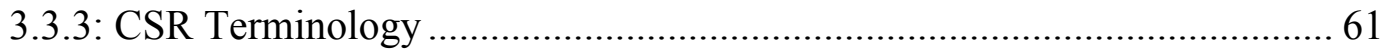

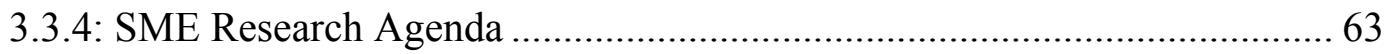

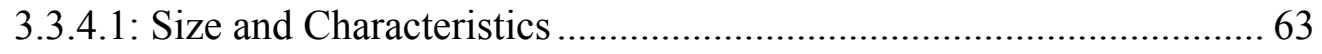

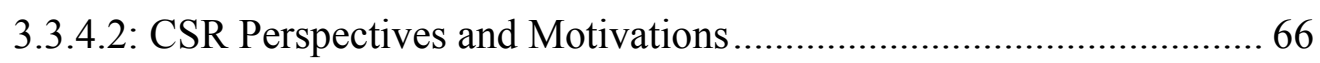

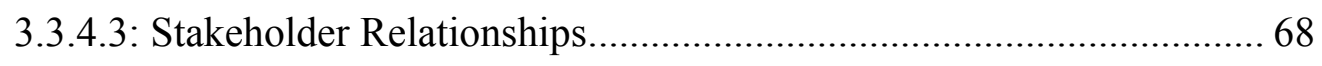

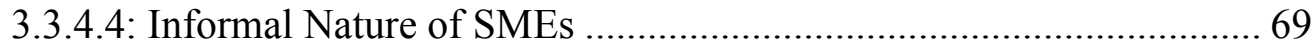

3.3.5: Theoretical Frameworks and Perspectives............................................... 71

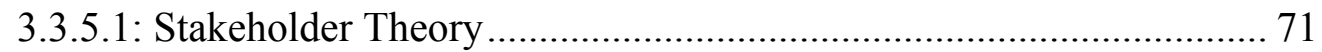

3.3.5.2: Small Business Social Responsibility (SBSR) Pyramids ................ 72

3.3.6: Analytical Perspective for CSR in SMEs ................................................ 74

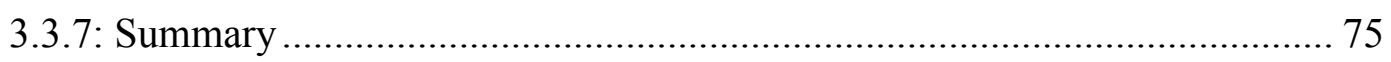

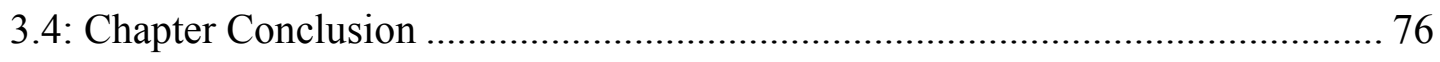

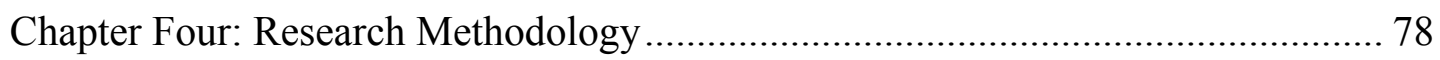




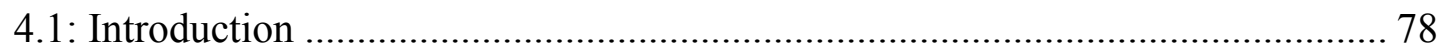

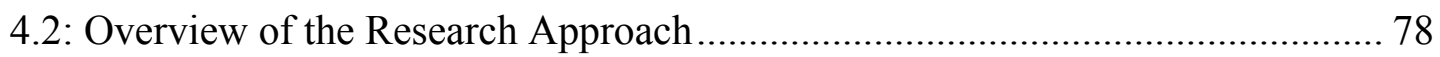

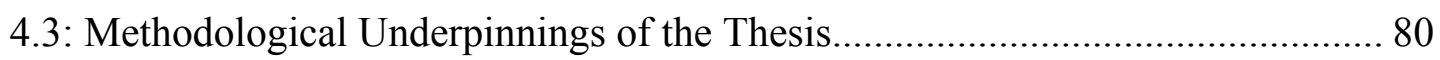

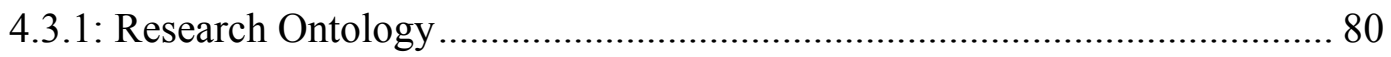

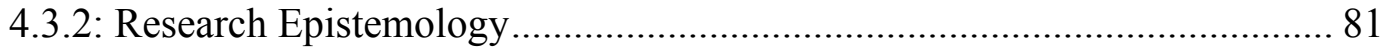

4.4: Research Perspective: Social Constructionism................................................ 84

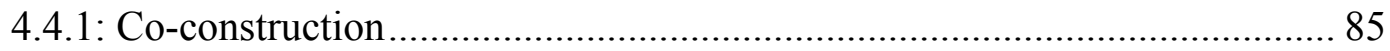

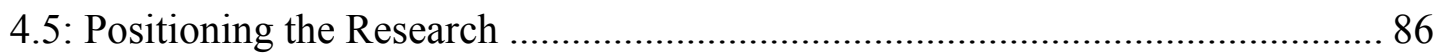

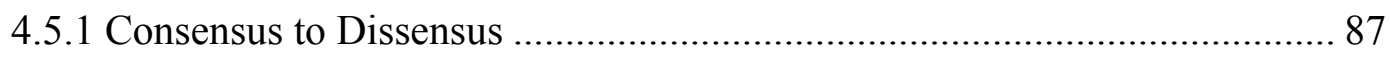

4.5.2: Local/Emergent to Elite/A Priori ........................................................... 88

4.5.3: Epistemological Position of the Research................................................ 89

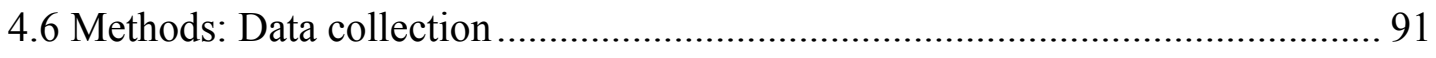

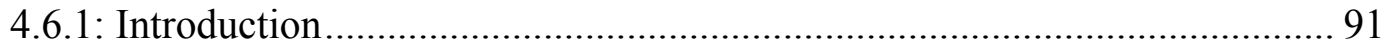

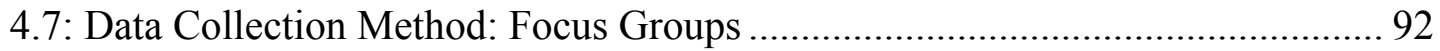

4.7.1 Data Collection Process: Focus groups ................................................... 93

4.7.1.1: Number of Focus Groups.......................................................... 93

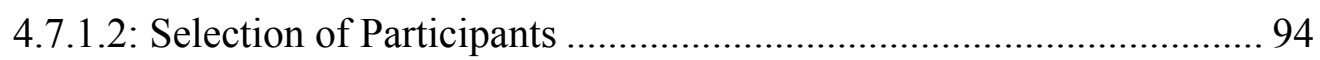

4.7.2: Focus Group Research Protocol................................................................ 96

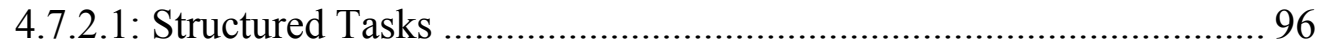

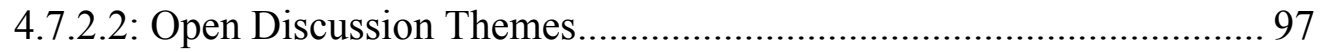

4.7.3: Focus Groups: Role of the Researcher...................................................... 98

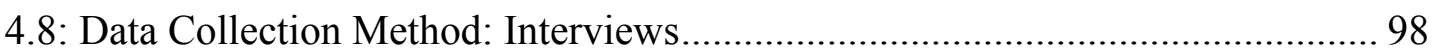

4.8.1: Data Collection Process: Interviews ........................................................ 99

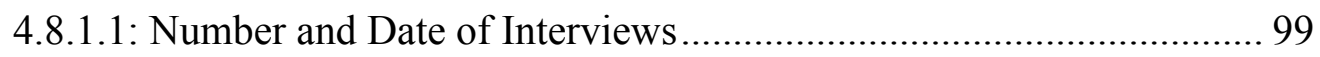

4.8.1.2: Selection of Participants ........................................................... 100

4.8.2: Interview Research Protocol ................................................................... 101

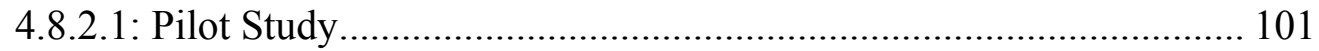

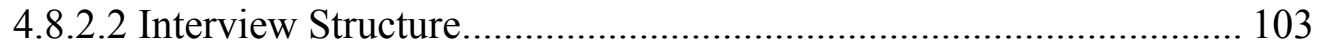

4.8.3: Interviews: Role of the Researcher ....................................................... 104

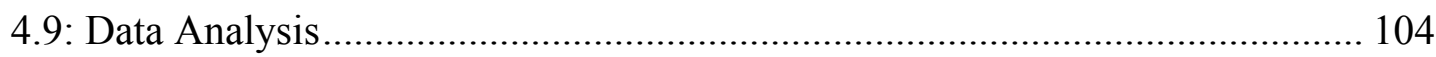

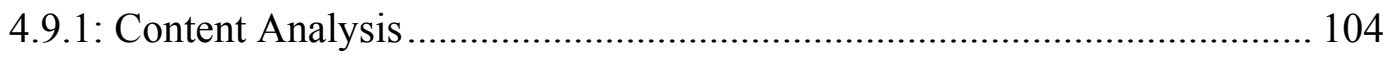

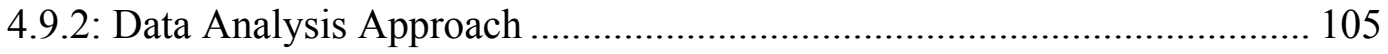

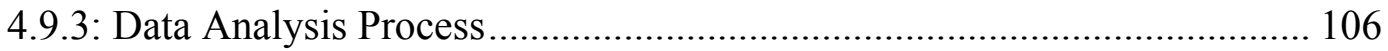

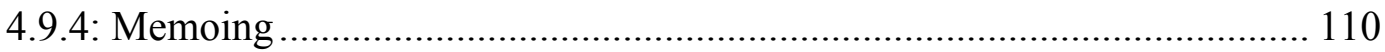


4.10: Socially Desired Response Bias

4.11: Validity

4.12: Reliability

4.13: Limitations and Boundaries of the Research ................................................. 114

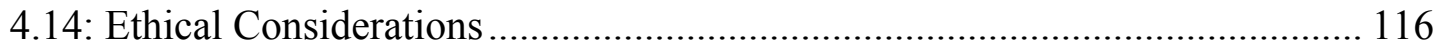

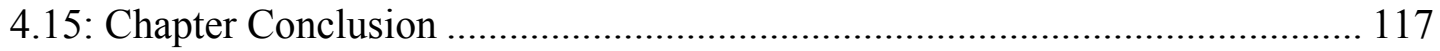

Chapter Five: Focus Group Findings - The Social Construction of CSR in SMEs 118

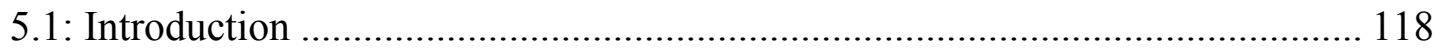

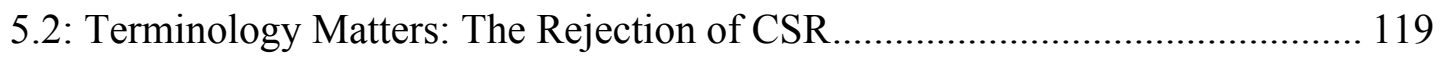

5.3: Social Identification: "More Socially Responsible as People" ........................ 121

5.4: Owner-Manager Values: An "Embedded Principle"....................................... 123

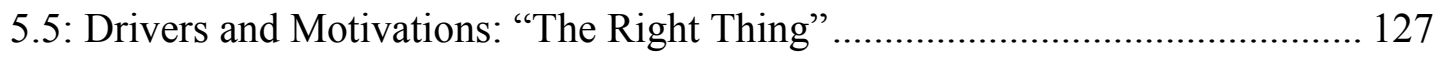

5.6: Community Embeddedness: The "Double Edged Sword" .............................. 129

5.7: Stakeholder Relationships: "An Element of Mutual Care” ............................. 132

5.8: Stakeholder Prioritisation: "Whoever is Banging at the Table Loudest" ......... 135

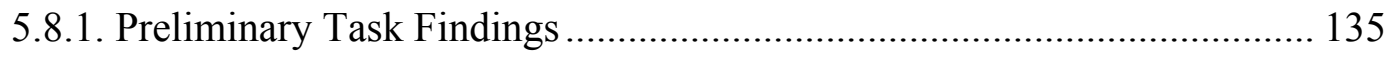

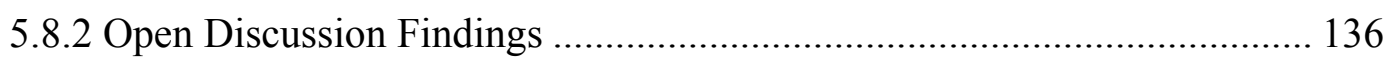

5.9. Barriers and Challenges: "A Big Change of Mindset" .................................. 138

5.10: Economic, Political and Societal Influences: "Blurred Boundaries" ............ 144

5.11: CSR Awareness: Disseminate and Educate.................................................. 149

5.12: CSR Impact: "The Planet and the People" ................................................... 152

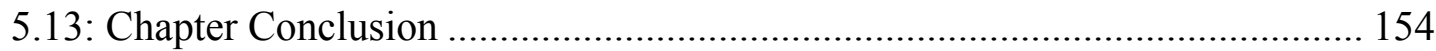

Chapter Six: Interview Findings (Micro - Individual level findings) ..................... 160

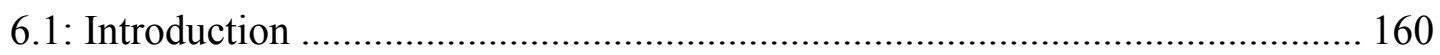

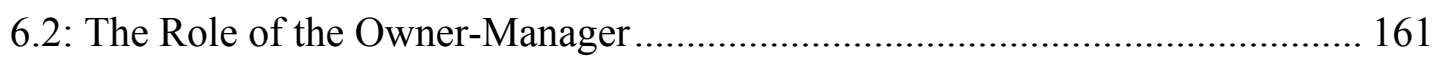

6.2.1: Owner-Manager Morals, Characteristics and Values ............................. 162

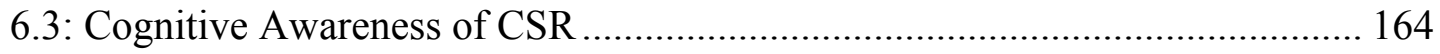

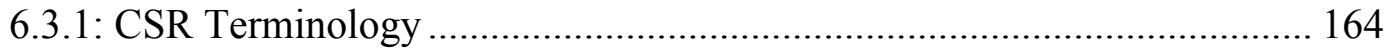

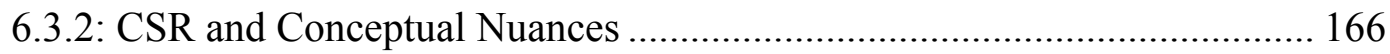

6.4: Individual Conceptualisation of SME Responsibilities................................. 172

6.4.1: Survival, Economic Contributions and Sustainable Business ................. 172

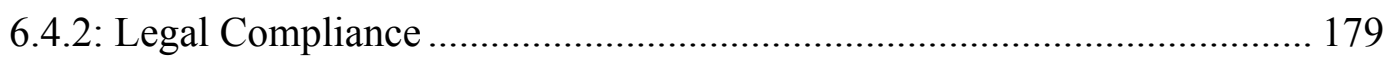

6.4.3: Ethical Standards, Norms and Expectations ........................................ 180 
6.4.4: Philanthropic Responsibilities

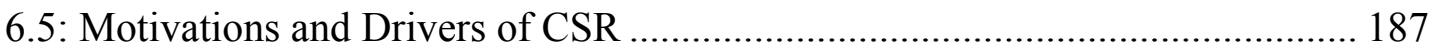

6.5.1: Intrinsic Motivations and Moral Obligations........................................ 187

6.5.2: Organisational Motives and Relational Drivers..................................... 194

6.5.3: Instrumental Drivers and Tangible Outcomes ......................................... 198

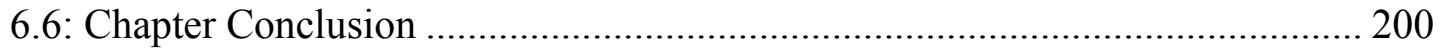

Chapter Seven: Interview Findings (Meso - Organisational level findings) ........... 202

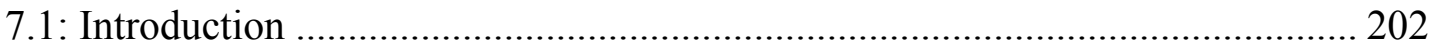

7.2: Characterisation of Stakeholder Relationships ............................................. 203

7.2.1: Family, Friendship and Long-Term Commitment................................. 203

7.2.2: Inextricable Personal and Business Connections................................... 208

7.2.3: Community Embeddedness, Reputation and Word of Mouth ................. 214

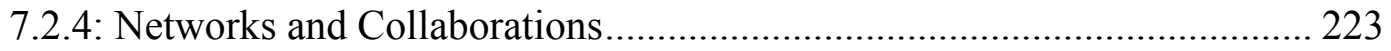

7.2.5: The Dynamic Nature of SMEs............................................................... 227

7.2.6: Stakeholder Relationships and the Balance of Power.............................. 230

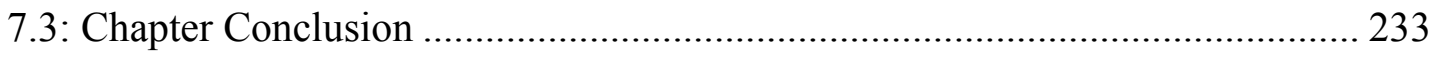

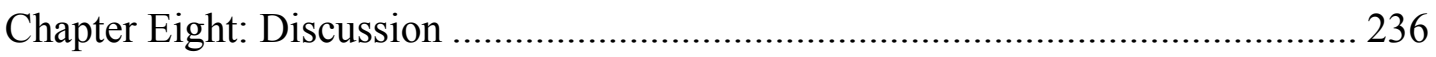

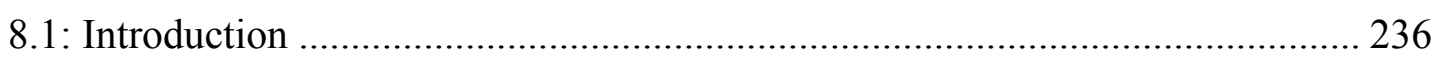

8.2: Corporate Social Responsibility (CSR) in Small and Medium-Sized Enterprises

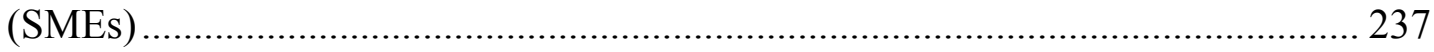

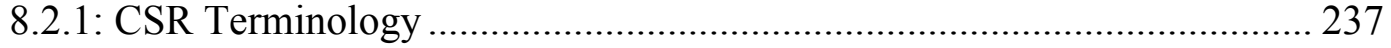

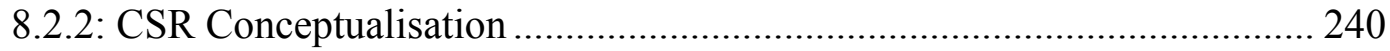

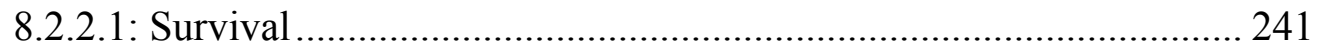

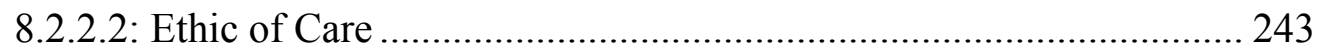

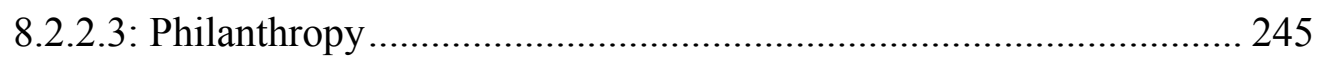

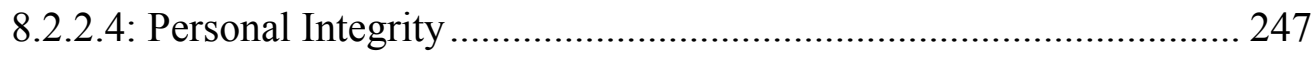

8.2.3: Spence's SBSR Pyramid Structure …...................................................... 249

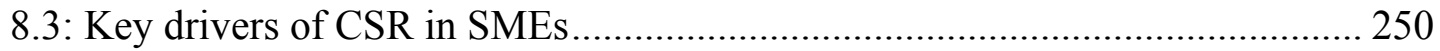

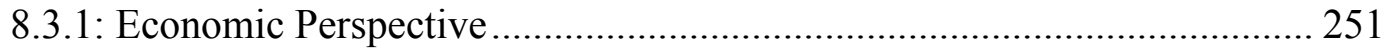

8.3.2: Relational/Organisational Perspective ................................................... 252

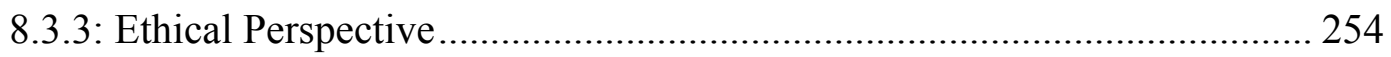

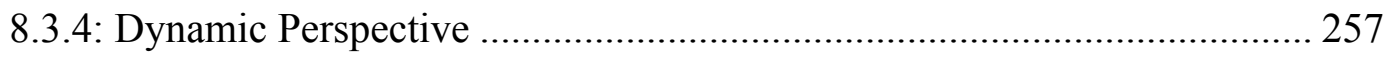

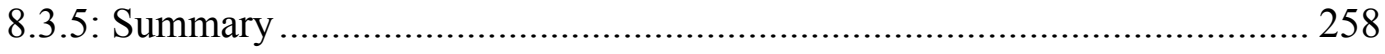




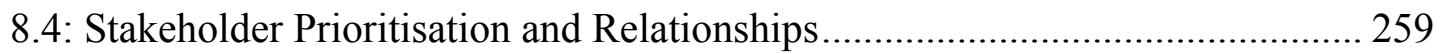

8.4.1: A Different Generalised Set of Stakeholders............................................. 259

8.4.2: Spence's Redrawn Stakeholder Theory .................................................... 261

8.4.3: The Nature of Stakeholder Relationships ............................................. 262

8.4.3.1: A Call for Care: Meeting the Needs of Others for Whom we Take

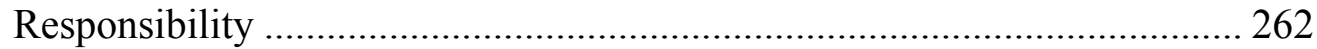

8.4.3.2: Inextricable Personal and Business Connections............................ 264

8.4.3.3: Networks and Collaborations....................................................... 265

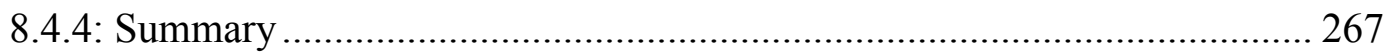

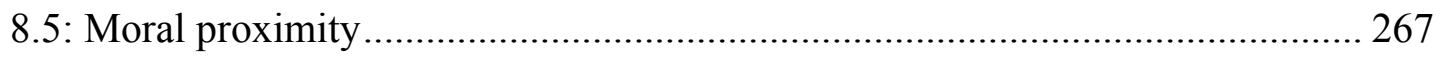

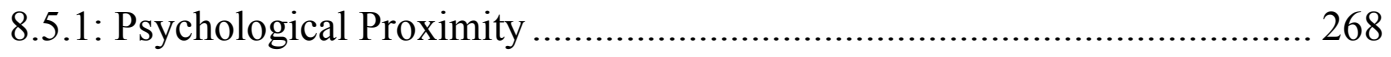

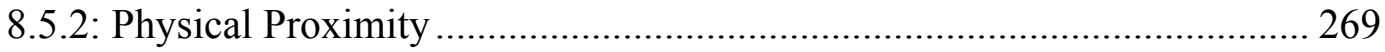

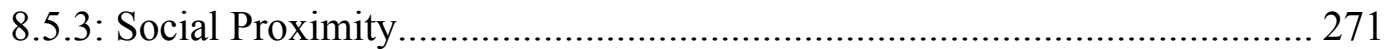

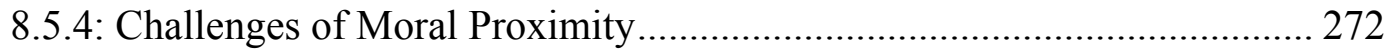

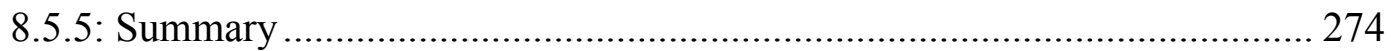

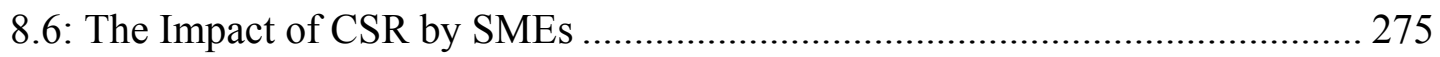

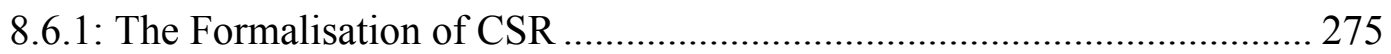

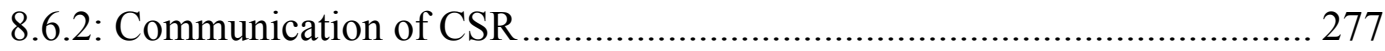

8.6.3: Institutionalisation of CSR by SMEs ……............................................. 278

8.6.4: Perceived Impact and Benefit of CSR by SMEs .................................... 279

8.6.5: Educating and the Dissemination of Knowledge .................................... 280

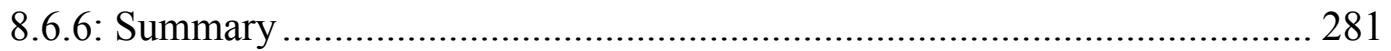

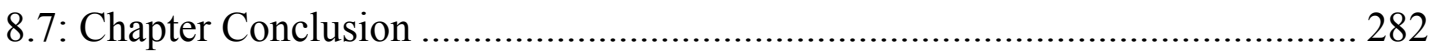

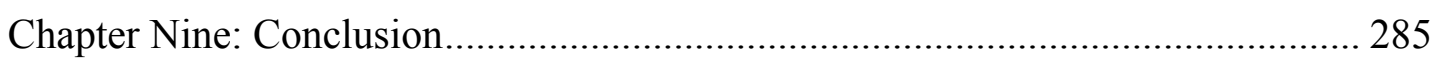

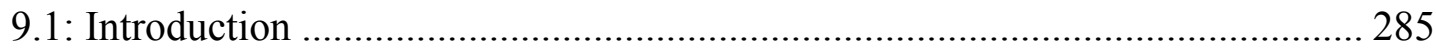

9.2: Revisiting the Research Aim, Objectives and Questions ................................ 285

9.3: Findings and Overview of the Research ..................................................... 285

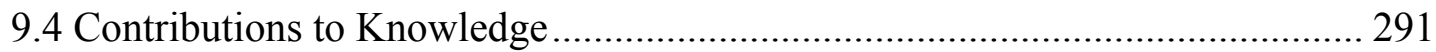

9.5: Limitations of the Study and Directions for Future Research ......................... 294

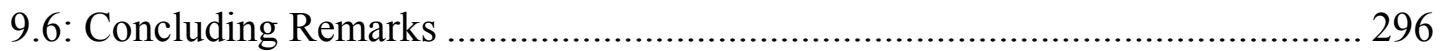

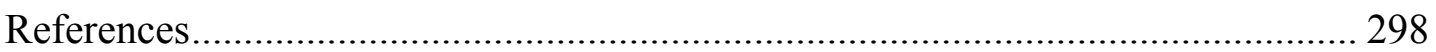

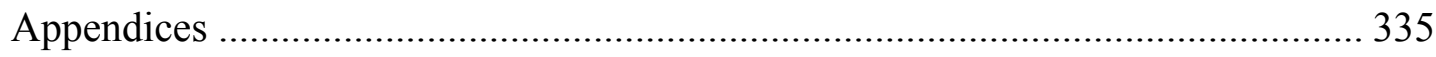




\section{Acknowledgements}

The years spent studying for this $\mathrm{PhD}$ have not only been invaluable for my personal development, but will hopefully make a small contribution to the important topic that is social responsibility. Whilst I claim full responsibility for the completion of this thesis, I cannot deny the support network that irrefutably believed in my ability and made it possible.

My heartfelt thanks go to Dr Simon Brooks, my first supervisor. Thank you for putting the wind beneath my sails and guiding my postgraduate journey, your kindness, continuous support and knowledge throughout each chapter significantly contributed to getting me across the finish line. You allowed me to accomplish something that I did not think possible and for that I will forever be in your debt.

I would like to extend my thanks to Dr Kate Organ, my second supervisor. Thank you for providing encouragement and feedback whenever possible, your input and support did not go unnoticed.

Special thanks must go to my colleagues and friends at Swansea University. The collective support and words of advice from my fellow $\mathrm{PhD}$ students and research group have intellectually challenged and made me a better academic researcher.

However, my main thanks must go to my family and friends. To my parents, Ruth and Matthew, and my sisters Holly and Lark, words cannot express how grateful I am for your love, encouragement and patience. I promise that I will make up for the time and energy sacrificed to this process. You constantly inspire me to be a better person and it is with love and pride that I dedicate this thesis to you. 


\section{List of Figures}

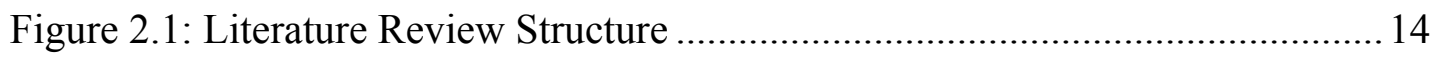

Figure 3.1: Chronology of CSR developments..................................................... 43

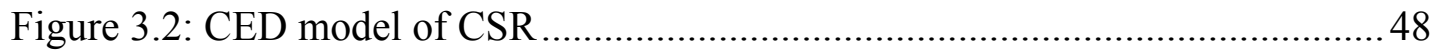

Figure 3.3: Carroll's (1979) Pyramid of CSR ….......................................................

Figure 3.4: Stakeholder Groups (Freeman, 1984) ....................................................52

Figure 3.5: Small business social responsibility (SBSR) pyramids ......................... 73

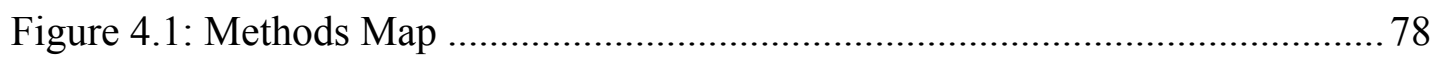

Figure 4.2: Dimensions from the Metatheory of Representational Practises ............87

Figure 4.3: Research Position on the Contrasting Dimensions from the Metatheory of

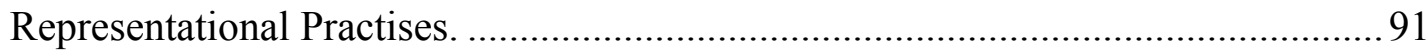

Figure 4.4: Focus Groups: The Geographical Distribution ..................................... 94

Figure 4.5: Criteria and Steps for Selecting the Research Sample ......................... 100

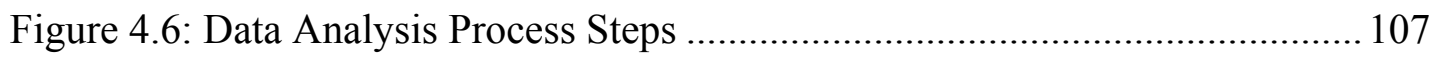

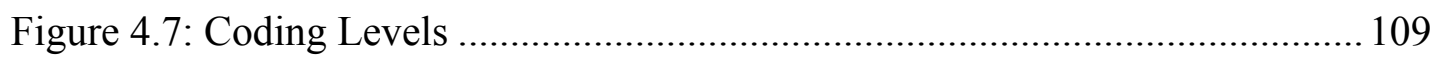

Figure 4.8: Memoing: The Layers of Thought Process ........................................... 111

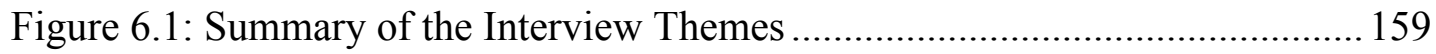

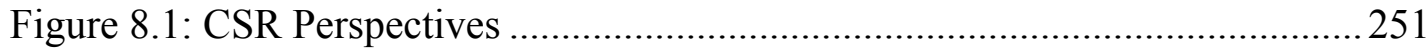

Figure 8.2: SME Key Stakeholder Map ..............................................................260

Figure 8.3: SME Key Stakeholders in 'Other' Category .......................................260 


\section{List of Tables}

Table 1.1: Research Questions and Objectives...................................................... 8

Table 2.1: Table of Ethical Theories and Relevant Literature...................................2 28

Table 2.2: Five Assumptions of The Ethic of Care .................................................. 35

Table 2.3: Corresponding Features of The Ethic of Care and CSR in SMEs.............36

Table 2.4: Jones' (1991) Constructs of Moral Intensity .............................................. 38

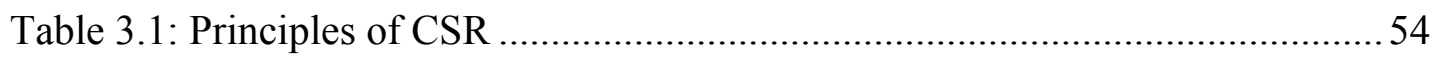

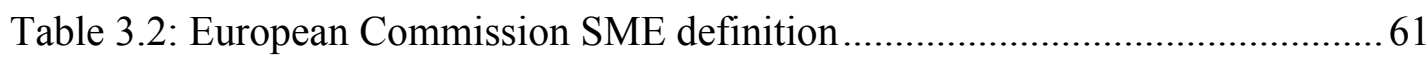

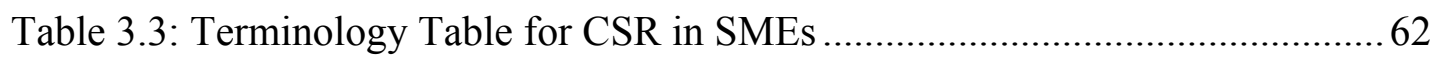

Table 3.4: Characteristic Differences between Large Corporate Firms and SMEs... 64

Table 3.5: Table of Comparative CSR Characteristics ...............................................65

Table 3.6: Four Enhancements of Stakeholder Theory for SMEs ............................ 72

Table 4.1: Key Differentials of a Positivist and Interpretivist Paradigm...................8 82

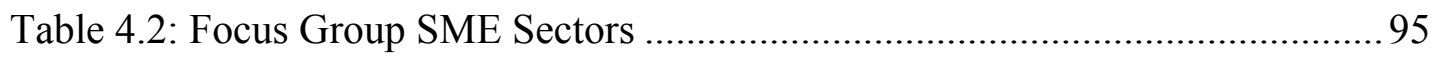

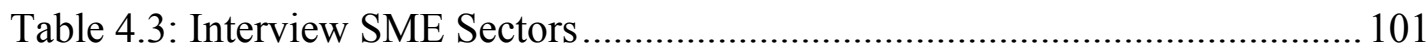

Table 5.1: Focus Groups: Summary of Super-Ordinate Themes............................ 119

Table 6.1: Interviews: Super-Ordinate Themes for Individual Level..................... 161

Table 7.1: Interviews: Super-Ordinate Themes for Organisational Level...............203 


\section{List of Appendices}

Appendix 3.1: Literature Overview: CSR in SMEs and Relevant Disciplines ....... 335

Appendix 4.1: FSB Regional Meetings and Focus Group Locations....................... 336

Appendix 4.2: Number of Focus Group Attendees ............................................... 337

Appendix 4.3: Focus Group SME Profiles ….......................................................... 337

Appendix 4.4: Percentage of Focus Group SME Sizes ............................................339

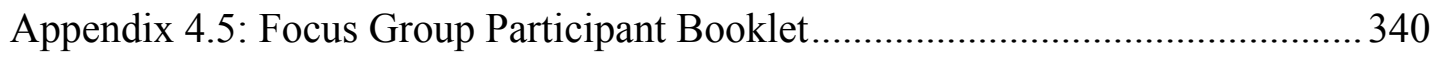

Appendix 4.6: Focus Group: Topics of Discussion and Supporting Literature........ 347

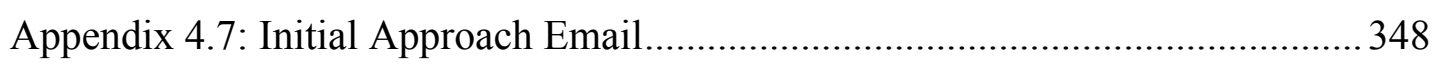

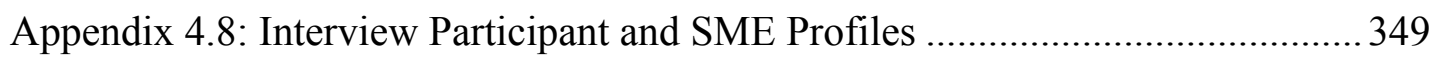

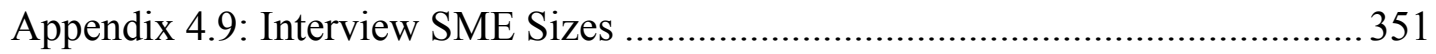

Appendix 4.10: Focus Group Themes and Interview Question Development Map 352

Appendix 4.11: Interview Themes and Questions...................................................353

Appendix 4.12: Raw Analysis Focus Groups: Content Analysis ............................ 357

Appendix 4.13: Raw Analysis Interviews: Content Analysis.................................. 358

Appendix 4.14: Raw Analysis: Table of Open Codes and Sub-Codes..................... 363

Appendix 4.15: Focus Group Coding: Data Visualisation ...................................... 364

Appendix 5.1: Focus Groups: Summary of Super-Ordinate and Sub-Themes.........365

Appendix 6.1: Interviews: Summary of Super-Ordinate and Sub-Themes ..............367

Appendix 8.1: System Orientated View of SME Stakeholder Theory .....................368 


\section{List of Abbreviations}

BITC - Business in the Community

CC - Corporate Citizenship

CED - Committee of Economic Development

CSB - Corporate Social Benevolence

CSR - Corporate Social Responsibility

CSO - Civil Society Organisations

CSV - Creating Shared Value

FSB - Federation of Small Businesses

MNC - Multinational Corporation

NGO - Non-governmental Organisation

SBSR - Small Business Social Responsibility

SMEs - Small and Medium-Sized Enterprises

UK - United Kingdom

UN - United Nations

US - United States of America 


\section{Publications from this Research}

\section{Peer-reviewed conference proceedings and workshop presentations}

Davies, F., Brooks, S. B., and Organ, K. (2015). Responding to the call for 'care' in CSR: A proposed study of small to medium sized enterprises. British Academy of Management Conference, Portsmouth University. September.

Davies, F. (2016). An exploration of small business social responsibility in small to medium-sized enterprises (SMEs). Postgraduate Research Student Symposium, Swansea University. May.

Davies, F. (2017). The Prioritisation of Responsibilities in Small to Medium-Sized Enterprises and the Influence of Moral Proximity on Ethical Behaviour. Postgraduate Research Student Symposium, Swansea University. June.

Davies, F. (2017). Moral proximity and small business social responsibility: An exploratory study of small and medium-sized enterprises. British Academy of Management Conference, Warwick University. September. 


\section{Chapter One: Introduction}

\section{1: Research Background}

The relationship between business and society is fundamental, yet complex. Societal expectations are changing and businesses are faced with increasing pressure to engage with responsibilities expanding past the economic (Jenkins, 2004). This thesis is located specifically in the field of corporate social responsibility (CSR) among small and medium-sized enterprises (SMEs). The growing significance of SMEs is progressively being recognised and has led to an emphasis on their social and environmental impact. In line with this recognition, the research asks how CSR is constructed and practised in SMEs, specifically looking at the role of moral perspectives to better explain the concept in this particular context.

CSR as an academic topic is multidisciplinary, drawing from fields such as economics, business management, strategy, entrepreneurship, business ethics, sociology and moral philosophy (Carroll, 2008; Carroll \& Shabana, 2010; Moore \& Spence, 2006; Spence, 2014). The competing and complimentary discourse provides a wide range of perspectives. However, this has resulted in a lack of consensus when defining the concept and a proliferation of alternative terms (Baden \& Harwood, 2013). The multifaceted and contested nature of CSR makes it inherently difficult to define, as Argandoña and von Weltzien Hoivik (2009) claim, "there is not - and probably cannot be - a unique, precise definition of CSR" (p. 221). As the social construction of CSR is a key focus of this research, a single definition is not suitable. Alternatively, a number of definitions are presented below in order to outline the scope of the CSR concept.

"Corporate social responsibility encompasses the economic, legal, ethical and discretionary (philanthropic) expectations that society has of organizations by society at a given point in time". (Carroll \& Buchholtz, 2014, p. 32)

"The firm's considerations of, and response to, issues beyond the narrow economic, technical, and legal requirements of the firm". (Davis, 1973, p. 312) 
"Corporate social responsibility (CSR) refers to companies taking responsibility for their impact on society". (European Commission, 2018)

"The social responsibility of business is to increase its profits". (Friedman, 1970)

In recent decades there has been a proliferation of CSR literature, gaining credibility in the academic and practitioner contexts (Carroll \& Shabana, 2010). A key feature of this proliferation is the focus on large organisations, routinely and systematically overshadowing any other form of business (Spence, 2016). The preoccupation with large firm CSR persists, despite the fact that globally, SMEs account for 95\% of private sector enterprises (International Trade Centre, 2015). This means that the important economic and social contribution of SMEs has been overlooked in much of the current literature (Spence, 2016). Historically, CSR studies that do focus on SMEs are characterised by the application of scaled, large firm theories (Jenkins, 2004; Russo \& Perrini, 2010). This has resulted in unfounded assumptions about the way that SMEs behave (Jenkins, 2006).

However, there have been a number of important contributions in recent years, with an increase in attention on SME specific research. The consideration of small business CSR as a separate category is now established in the field (Moore \& Spence, 2006; Spence, 1999, 2004; Spence \& Painter-Morland, 2010; Vives, 2006). However, whilst there has been a significant rise in descriptive research, there is still a distinct lack of theoretical developments that start from this point of view. Without a theoretical basis to bridge the gap between SME and mainstream CSR literature, academic and practitioner credibility will not be gained (Spence, 2007).

There are two key observations of the current CSR literature for SMEs that limit theoretical development. The first is that it lacks context sensitive approaches relevant for SMEs, and the second is that it is characterised by a preoccupation with proving the business case. It is widely acknowledged in the literature that SMEs are heterogeneous, yet "decontextualisation is a particularly common problem", whereby theorisation fails to acknowledge "specific characteristics, relationships and attributes of small businesses (Lähdesmäki, Siltaoja \& Spence, 2017, p. 3). This 
problem is widely recognised by small business researchers (Jenkins, 2004, 2006; Lähdesmäki \& Suutari, 2012; Spence, 2016), with Holliday (1995) claiming, "small firms tend to be homogenised by quantitative studies". He suggests, "only a qualitative approach responds to the differences within and between firms" (p. 174).

Furthermore, through a broad review of the literature, it has been recognised that the CSR concept has been progressively constricted by economically rational justifications. This could be afforded to the current obsession with establishing a link between CSR and organisational success. This viewpoint has institutionalised and co-opted CSR as a managerial activity and strategic tool for organisations (Baden \& Harwood, 2013). In response to this, a large proportion of the small business literature calls to reclaim the moral foundations of CSR in SMEs (Brooks, 2010; Baden \& Harwood, 2013; Baden, 2016; Nijhof \& Jeurissen, 2010; Spence 2014, 2016).

Spence (2016) argues that a refocus on the social sciences is more important than advancing the business case for SMEs. This is because the emphasis on strategic and instrumental outcomes "drives out the intrinsic motivation for engaging in CSR" (Nijhof \& Jeurissen, 2010, p. 618), and because profit maximisation is generally not the main priority of SMEs. She claims that policy makers should "take heed of the need to understand the ethical character of the small firm and avoid one-dimensional programmes relating social responsibility to profit-maximization" (Spence, 2014, p. 386). Baden and Harwood (2013) further this by claiming that the term CSR "has strayed from the focus on societal good" (p. 617), identifying a need to address the subordination of morality because "once CSR loses its foundation in ethics it becomes not only irrelevant, but counterproductive" (p. 617).

The above observations call for suitable theories and methods that are contextually sensitive and acknowledge the role of morality in CSR. There are ongoing debates in the ethics field as to the most suitable theoretical perspectives to do this. Although, it could be recognised that currently there is not one theory that adequately explains CSR in the SME setting, with even the ethics domain dominated by theories supportive of the rational approach. This identifies a substantial gap in the current research agenda. 
Nevertheless, recent research by Baden (2016) does acknowledge the need to "provide a moral counterweight to economic forces" (p. 1) and presents a theoretical reconstruction of Carroll's CSR pyramid, redefining it as a predominantly ethical concept. Spence (2016) further attends to the need for size sensitive research and offers, "appropriately crafted theory" (p. 45) for empirical testing and further development. She is one of the only academics to redraw CSR theories with increased relevance for SMEs, arguing specifically for the importance of two moral perspectives: moral proximity and the ethic of care (Spence, 2014).

Moral proximity has been identified as a moral perspective that does acknowledge context. It is a relevant construct for CSR in SMEs due to distance being "the manner in which human beings view moral issues in respect of physical, psychological, cultural and social parameters" (Spence, 2014, p. 382). These parameters are what links responsibility to the owner-managers and stakeholders closest to them physically and emotionally, influencing the salience of moral issues. The ethic of care, a previously marginalised theory, compliments moral proximity by explaining the behaviours and practises resulting from different classifications of closeness. It does not focus on principles, outcomes or processes like traditional theories, but relationships and context.

Both of the moral perspectives above are claimed to be central to progress the CSR debate in SMEs and address gaps in the literature. However, whilst Spence (2016) has provided the appropriately crafted theory, there has not yet been empirical testing. It is this gap in the literature that the following research addresses. Therefore, this thesis is one of the very few studies that have empirically and qualitatively explored the ethic of care and moral proximity in relation to CSR in SMEs.

\section{2: Theoretical Framing}

By locating this thesis in the sociology of economic behaviour and field of business ethics, it acknowledges the wider context in which CSR and related fields occur. As 
the above highlights, there is a need for practical and theoretical progressions reclaiming the moral foundations of CSR and explaining the socially responsible behaviour of SMEs. It is sociological theories that provide the foundations for ethical decision-making and assist a deeper level of comprehension for CSR in SMEs. This is important when considering the social construction of CSR.

By drawing from specific aspects of the sociology of economic behaviour, it allows focus on two key concepts of this thesis: economic rationality and morality. Contributions from the sociological discourse imply that ethical acts cannot be dealt with in isolation to their context and propose the view that morality has been displaced when economic rationality is applied in the wrong place. This field views economic activity as being a social activity and therefore regards sociological analyses as relevant for the study of CSR. The tensions between these constructs are central to addressing gaps in the current research agenda.

Another reason to locate this thesis in reference to these fields is that ethical theory is the primary source of explanatory concepts. It is therefore important to understand the theories that frame CSR justifications. However, the literature identifies that there has not previously been an ethical theory suitable for SMEs that adequately acknowledges the role of the individual and context. It has been argued that even the domain of ethics is dominated by a problematic reliance on abstract rules and theories supporting the rational approach (Held, 1990; Spence, 2014; Spence, 2016). Business ethics in its present form has been described as "at best window dressing and at worst a calculated lie" (Jones, Parker \& ten Bos, 2005, p. 1).

Spence (2014) extends this view and considers that without acknowledging moral perspectives, "normative judgements about behaviour are being made in a moral vacuum with only a popular, at worst journalistic, interpretation of ethics" (p. 381). Consequently, there is room for new or previously marginalised theories to be incorporated into the research agenda. By recognising both of these fields, this thesis aims to adopt an in-depth approach that evaluates the relationship between business and society on multiple levels. In doing so, this contributes not only to knowledge on CSR in SMEs, but the relevance of ethical theories and role of the sociology of economic behaviour. 


\section{3: Theoretical Position}

The following research adopts a social constructionist perspective. As claimed by Spence, Schmidpeter and Habisch (2003), "business ethics does not operate in a vacuum disconnected from the rest of the world" (p. 13) and it is not possible to separate actors from their environment or relationships previously formed within it. Fundamental to the social constructionist perspective is the acknowledgement of context and commitment to establishing the meaning of behaviour from the perspective of the actor under enquiry. Alvesson and Deetz (2000) explain this view whereby CSR is related to a "wider cultural, economic and political context" (p. 1) in which it is constructed. With regards to the sociology of economic behaviour, MacIntyre (1981, 1985) and Polanyi (1944/2001) are drawn upon to emphasise the importance of context, as is the ethic of care and moral proximity as appropriate ethical theories to reflect the contextual composition of SMEs (Spence, 2016).

In order to address the main research aim and objectives outlined below, the study followed an exploratory qualitative research strategy to allow for rich exploration and a comprehensive evaluation of CSR. The research draws from the interpretive repertoires of SME owner-managers in order to uncover differing interpretations of CSR and significant factors that influence inception and engagement (Potter, 1996). This opened up enquiry into the role and relevance of moral perspectives. These interpretations were established via four exploratory focus groups and thirty in depth interviews with SME owner-managers from diverse sectors. The interviews were transcribed and then analysed, informed by a priori codes and an inductive content analysis. Findings of the focus groups were studied according to a multidimensional framework of analysis; accounting for contextual factors from individual, organisational and institutional levels. This allowed a holistic view of CSR and the establishment of conceptual boundaries. The interview findings were then analysed in relation to two specific levels in line with the focus of the research.

\section{4: Practical Context}


The practical context for the primary data collection of this thesis was the UK. The reasons behind this choice were twofold. Firstly, the SME sector provides a vital foundation for the UK economy. According to the Department for Business, Energy and Industrial Strategy (2017), there were just over 5.7 million private sector small businesses in the UK, with $99.9 \%$ classified as SMEs. These accounted for $60 \%$ of all private sector employment in the UK, providing 16.1 million jobs. These figures alone emphasise the importance of this growing sector. A second reason for conducting the study in this context is because the UK is conducive to CSR (Spence $\&$ Perrini, 2009), with a political focus on raising awareness and creating a suitable environment that encourages adoption of social responsibility initiatives. The UK government recognise that the SME approach to CSR is distinct from large firms, endeavouring to accommodate this. The above factors point towards the UK context being a proliferant ground to research the relationship between business and society.

Additionally, whilst the UK was the broader practical context of this research, a large proportion was conducted in Wales. Arguably, this setting has heightened relevance for CSR. This is again due to the economic reliance of SMEs for the Welsh economy and community culture embedded in rural regions. Whilst SMEs account for $61.7 \%$ of employment similar statistics to the UK, these figures are still increasing. Micro enterprises alone constitute $94.9 \%$ of all enterprises and have shown the largest increase in employment (Statistics for Wales, 2017). However, one of the most interesting aspects of the Welsh context is the introduction of The Wellbeing of Future Generations Act (2015). This act legally requires all public bodies to consider social, cultural, environmental and economic wellbeing and reinforces the long-term impact of their decisions. Despite this policy specifically targeting public bodies, the act will commonly be referred to and impact SMEs as it cascades down the supply chain.

The reliance on SMEs and political focus on the long-term wellbeing of Wales provides an exciting context to study the small business conception of CSR. The Wellbeing of Future Generations Act (2015) is pioneering and the first of its kind. This has positioned Wales as a site for significant public investment and worldwide attention, with international recognition from the United Nations (UN). This act alone signifies the ambition of Wales to consider and address the social, cultural, 
environmental and economic sustainability of the country and those businesses within.

\section{5: Research Aims and Objectives}

In alignment with the gaps and unanswered questions identified above, the broad aim of this research is to develop an understanding of how CSR is constructed and practised in SMEs and the role of moral perspectives to explain such engagement. Whilst there are a multitude of questions that would have relevance for this thesis, the following has been selected in order to bring an element of focus, whilst still allowing for rich exploration:

How is CSR constructed and practised in SMEs and what role do moral perspectives play to explain such engagement?

To ensure that the research question is fulfilled, the following objectives and questions were formulated (see Table 1.1). Mindful that an element of this research is emergent, they are not intended to offer a prescribed structure, but loose guidelines to focus the research.

Table 1.1: Research Questions and Objectives

\begin{tabular}{|l|l|}
\hline Research Objectives & Research Questions \\
\hline $\begin{array}{l}\text { RO1: To evaluate how SME } \\
\text { owner-managers construct and } \\
\text { make sense of their role in society }\end{array}$ & $\begin{array}{l}\text { RQ1: How do SME owner-managers } \\
\text { conceptualise and make sense of their } \\
\text { responsibilities towards society? }\end{array}$ \\
$\begin{array}{l}\text { RQ2: Is the ethic of care a relevant theory to } \\
\text { explain the CSR conception of SME owner- } \\
\text { managers? }\end{array}$ \\
$\begin{array}{l}\text { RO2: To investigate the key } \\
\text { motivations and drivers for CSR } \\
\text { in SMEs }\end{array}$ & $\begin{array}{l}\text { RQ3: What are the key motivations and } \\
\text { drivers for CSR engagement in SMEs? }\end{array}$ \\
\hline RO3: To explore CSR & RQ4: On what basis do SMEs prioritise \\
\hline
\end{tabular}




\begin{tabular}{|c|c|}
\hline $\begin{array}{l}\text { engagement within SMEs and the } \\
\text { role of key stakeholders in } \\
\text { shaping such practices }\end{array}$ & $\begin{array}{l}\text { stakeholders and what influence does this } \\
\text { have on CSR engagement? } \\
\text { RQ5: How does the nature of relationships } \\
\text { with key stakeholders influence the } \\
\text { inception and engagement of CSR in SMEs? }\end{array}$ \\
\hline $\begin{array}{l}\text { RO4: To explore the role that } \\
\text { moral proximity to key } \\
\text { stakeholders can play in } \\
\text { influencing or inhibiting CSR } \\
\text { practices in SMEs }\end{array}$ & $\begin{array}{l}\text { PQ6: Does moral proximity to stakeholders } \\
\text { influence the conceptualisation of CSR for } \\
\text { SMEs? } \\
\text { PQ7: Does moral proximity to stakeholders } \\
\text { influence the inception and engagement of } \\
\text { CSR? }\end{array}$ \\
\hline $\begin{array}{l}\text { RO5: To summarise the } \\
\text { perceived and potential impact of } \\
\text { SME CSR initiatives }\end{array}$ & $\begin{array}{l}\text { RQ8: What influence does the } \\
\text { conceptualisation and engagement of CSR } \\
\text { in SMEs have on broader societal } \\
\text { wellbeing? } \\
\text { RQ9: How can the promotion of CSR in } \\
\text { SMEs be supported in the future? }\end{array}$ \\
\hline
\end{tabular}

\section{6: Research Boundaries}

As previously explained, the research evaluates CSR in SMEs from the perspective of the owner-manager. The social construction of CSR is therefore placed on the intrinsic processes of one individual, despite the desire to evaluate the CSR perspective of the whole organisation. As such, this may not be a fair representation of the SME as a whole. Additionally, the following study does not seek the perspectives of recipients of CSR. The opinions of those benefiting from SME engagement could provide valuable perspectives on the perceived impact of social initiatives, yet are not currently represented in the research agenda. Finally, the research is confined to the UK geographical context and adopts a loose SME definition. Cross-country comparisons would have been warranted to identify differences between the UK and other contexts, or more focused studies distinguishing between variables such as size, or cross-sector analysis of the findings. 


\section{7: Research Contributions}

This research offers a number of potential contributions. The first is the empirical exploration of CSR in SMEs in the UK context. The study adopts a social constructionist approach that considers CSR as a socially centred phenomenon, reflecting the reality of these SMEs. Acknowledgment of moral philosophy and the sociology of economic behaviour as foundational constructs of CSR also allows for a deeper level of comprehension. Second, with a renewed interest into the influence of context and relationships, it urged the researcher to evaluate the CSR phenomenon via two theoretical lenses. This is one of the first studies that empirically explores the role of the ethic of care and moral proximity in relation to CSR in the SME context. The research adopts two redrawn CSR theories through these perspectives, making practical suggestions to enhance Spence's proposed stakeholder theory and small business social responsibility pyramids (SBSR). These suggestions are in line with the empirical observations. Third, alongside theoretical contributions, the research findings provide guidance for support institutions, policy makers and practitioners in the UK and Wales to further accommodate the SME conception of CSR. This information will be important with the increasing pressure on smaller businesses to formalise their CSR engagement.

\section{8: Structure of the Thesis}

The following section will summarise the structure of the thesis with a brief synopsis of the chapters to follow. The research is structured systematically over nine chapters. Following this introductory chapter, these comprise of a literature review, research methods, research results, discussion and a conclusion.

Chapter Two considers literature from the sociology of economic behaviour and brings ethical theories to the foreground of discussion. This draws from sociological constructs such as economic rationality and morality to explain the relationship between business and society. Specific contributions come from the likes of Max Weber, Emile Durkheim and Karl Polanyi, as well as additional inputs from Alasdair MacIntyre and Ralph Fevre. The evaluation of this literature is important to 
understand the tensions between the constructs and the role that this had on the historical development of CSR. Following this, ethical theories were brought to the foreground of discussion, with this chapter providing an overview of the ethical theories commonly adopted in CSR research. A key aim was acknowledging the multiple ways that ethics can be assessed and to establish the relevance of different theories for framing CSR in SMEs.

Having explored the broader sociological and ethical context of CSR in SMEs, Chapter Three turns to the chronological development of the CSR literature. Key themes and progressions were acknowledged to map the CSR landscape and show how the concept has evolved historically. In establishing CSR in SMEs as an area requiring further investigation, the chapter then moves to the SME definition adopted for the research and highlights the significance of SMEs. Following this, attempts were made to define the CSR concept for SMEs specifically, despite a lack of one unified term. By outlining the current SME specific research agenda for CSR, themes were then picked up on relevant to the research. For example, the lack of a unified definition, the distinct size characteristics of SMEs affecting the inception and engagement of CSR, a preoccupation with establishing the business case, a lack of theoretical developments that acknowledge the context of SMEs and finally methodological weaknesses in past literature. In conclusion, the chapter identifies two moral perspectives suitable to theoretically progress the research agenda for CSR in SMEs and to guide the research to follow.

Chapter Four presents and defends the research methodology, strategy and methods adopted to collect and analyse the data. The chapter begins with a clear overview of the research approach and methods adopted, followed by a more detailed discussion and defence of the methodology and methods. This starts with an exploration of the ontological and epistemological position of the research, acknowledging how the positioning evolved as the research developed. The chapter progresses to discuss the data collection methods of focus groups and interviews, followed by clarification of the content analysis technique used for data analysis. The chapter concludes by identifying the role of the researcher and considering validity, reliability and ethical considerations of the research. 
Having presented and defended the appropriate research methods, Chapter Five is the first of the research findings chapters. This chapter presents and analyses the preliminary findings from the exploratory focus groups. These produced interesting empirical data in their own right. The emergent themes derived from the focus groups informed the approach adopted for the main phase of data collection and had an integral role to structure and contextualise the findings that followed.

Chapter Six and Chapter Seven then turn to the interview findings. These are separated into the individual and organisational level findings to accommodate a multi-layered approach. This allows for a fuller comprehension of the concept to accommodate the context in which SMEs are embedded. Content analysis is utilised to draw themes from the data and to begin to evaluate how CSR is socially constructed in SMEs. The main focus of the chapters was first to understand how CSR is conceptualised and made sense of on an individual level and second to understand the organisational context and networks of stakeholder relations that influence and affect the uptake of CSR.

Following the preceding chapters, Chapter Eight interprets the findings and discusses them in comparison to previous literature. This is in order to establish what the findings confirm or uncover about the nature of CSR in SMEs and to determine the relevance of moral perspectives. This chapter is structured in line with the key research objectives, presented with extracts of text interposed with interpretive analysis.

To conclude, Chapter Nine provides an overview of how the research questions have been addressed and makes clear the key contributions to knowledge made by this work. This is followed by a reflection on the boundaries of the work, identification of directions for further research and finally some concluding remarks.

\section{9: Summary}

The introductory chapter of this thesis began with a brief overview of the research. This outlined the scope of the study and identified relevant research problems that 
warranted further exploration. This was followed by consideration of the theoretical position and framing of the research. The main research aim, objectives and questions were then specified to bring focus to the study, with potential boundaries and key contributions outlined subsequent to this. To conclude, an overview of the thesis structure and content was presented. Having now established the research aim and objectives, Chapter Two will turn to a more detailed analysis of the literature. 
Figure 2.1: Literature Review Structure

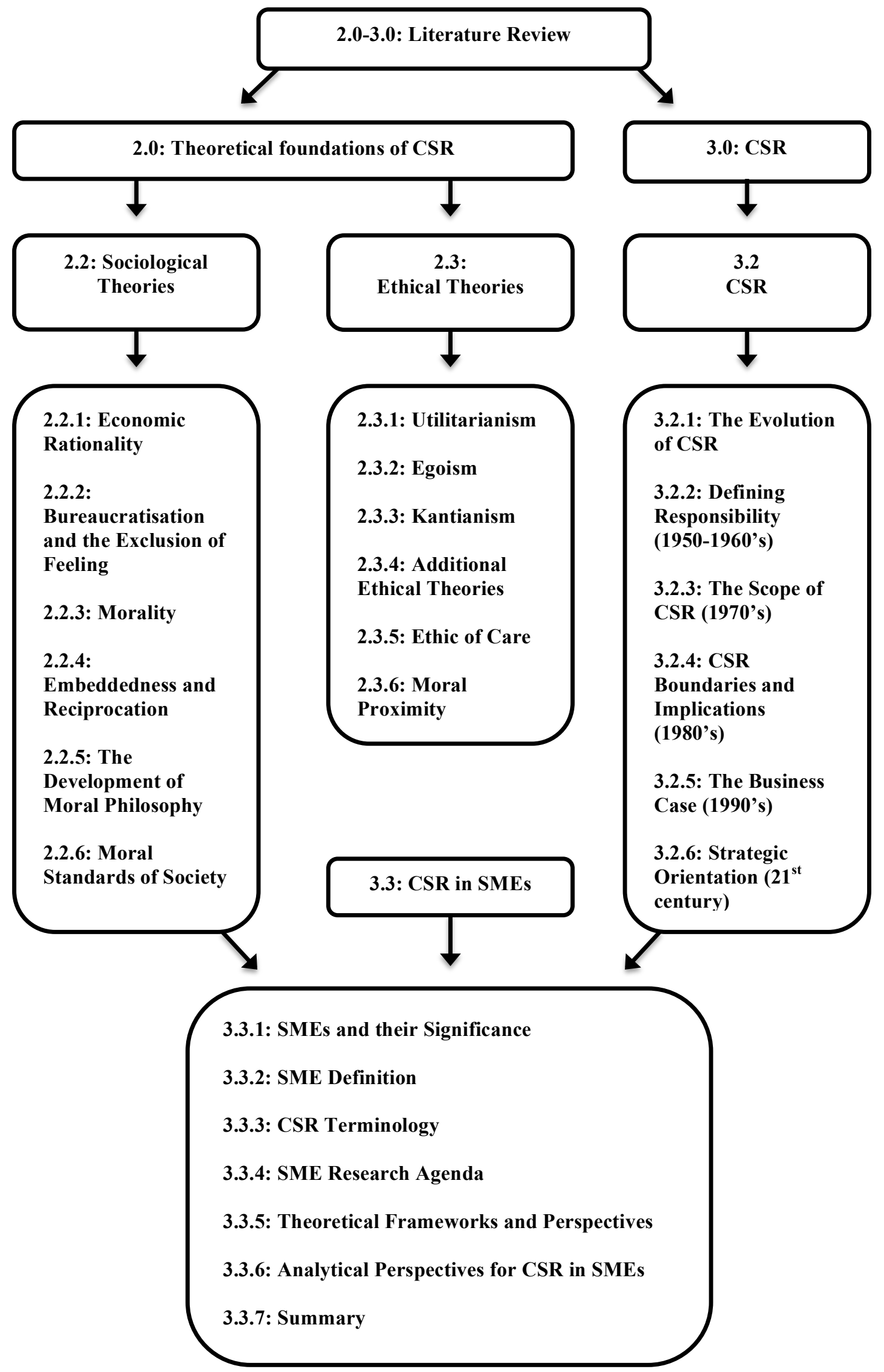




\section{Chapter Two: Literature Review - Theoretical Foundations of CSR}

\section{1: Introduction}

Having established the research aims and objectives in Chapter One, this chapter locates the thesis in terms of the sociology of economic behaviour and field of business ethics. This allows for acknowledgment of the wider context in which CSR has occurred. The chapter begins with an analysis of economic rationality and morality, as two sociological constructs relevant for the development of CSR (section 2.2). Following this, focus then turns to ethical theories, particularly identifying those that have dominated the business and society literature to date (section 2.3). Attention then narrows to consider the theories adopted to frame CSR in SMEs specifically, with weaknesses in the current theoretical perspectives and areas of opportunity identified. Section 2.2 and 2.3 are both concluded with detailed summaries, with section 2.4 providing an overall conclusion of the chapter.

\section{2: Sociological Theories}

The first section of this chapter will consider the sociological understanding of business and society relations to date. Such theories provide the foundations for ethical decision-making and assist a deeper level of comprehension for CSR in SMEs. Economic rationality and morality are key topics of this thesis to provide exploration of the sociological constructs behind CSR and relevant theoretical progressions. Therefore, the major works of Max Weber, Emile Durkheim, Karl Polanyi and Alasdair MacIntyre will be included in this review, drawing from more recent adaptations to these theories such as those from Fevre and Parker. $\mathrm{s}$

\subsection{1: Economic Rationality}

Economic rationality is an important consideration for this thesis. One of the main research objectives is to establish how CSR is made sense of and understand if SMEs view CSR as an economic or moral construct. To do this, a number of 
sociological themes will be outlined in order to understand what economic rationality is and how it can influence CSR in SMEs.

The task of defining economic rationality is difficult due to the ambivalence of this type of rationality. That being said, in order to clarify for the purposes of this thesis it can be described as "the sub-category of rationality which says life is all about economics and economics is all about a particular way of calculating means and ends" (Fevre, 2003, p. 6). The concept is founded on the idea "that everyone is out for themselves and interested in maximizing their own pleasure and minimizing their experience of pain" (Fevre, 2000, p. 200), with sales, profit and efficiency dominating the lexicon.

The concept was first labelled "practical reasoning" (Fevre, 2000, p. 4) similar to Weber's 'practical rationality' (Kalberg, 1980, p. 1151), but later progressed to the term economic rationality. There are numerous types of economic rationality that permeate Max Weber's work due to the polymorphous character of rationality. However, his specific contribution to this research is that of rational actions with regard to practical rationality, as opposed to theoretical, formal or substantive (Kalberg, 1980, p. 1146). This is one of Weber's constructions focusing on the individual and their egotistic interests suggesting that:

"A practical rational way of life accepts given realities and calculates the most expedient means of dealing with the difficulties they represent." (Kalberg, 1980, p. 1152)

In other words, it regards the most efficient way of getting to the most adequate means. He saw this as the most dominant in the industrial era.

Weber shaped the characteristics of modern occidental capitalism that is often linked to practical rationality. His first concern was to explain occidental rationalism and the peculiarity of it, clarifying that "every such attempt at explanation must, recognising the fundamental importance of the economic factor, above all take account of the economic conditions" (Weber, 1930/2003, p. 26). This arguably was key to the acceleration of economic rationality and why Weber has been adopted as the founder. 
Rationalisation and individualisation have remained prominent themes of modern economic life (Sayer, 1991), with production and profit at the forefront of goals. In the foreword of Weber (1930/2003) Tawney describes rationalisation as "a term of art, to describe an economic system base, not on custom or tradition, but on the deliberate and systematic adjustment of economic means to the attainment of the objective of pecuniary profit" (p. 1e). Despite Weber believing that rationalism is an inescapable fate in modern society, he does not blame capitalism for the selling of labour, but the rationalisation present in production.

Weber's view was that rationalisation; particularly economic rationality was awakened due to the rise of capitalism and privatisation. He suggests that it was used to relieve dependence upon the natural limitations of the human individual. As Zouboulakis (2001) summarises, Weber uses "mental constructions as a theoretical model to explain - or to 'understand' - in individualistic terms the real world inhabited by real persons in a given historical and geographical context" (p. 30). This highlighted the prominence of individualism within his theories when talking about economically rational actions.

Another important concept from Weber is 'the calling', with a religious connotation appearing from Protestantism. This is the view that the highest form of moral obligation and "the only way of living acceptably by god" is "through the fulfillment of obligations imposed on the individual by his position in the world" (1930/2003, p. 81). Weber used 'the calling' as an attempt to find out where the concrete form of rationality came from. He asked why in the spirit of modern capitalism is profit an end in itself and the pursuit of it considered virtuous. With guidance from the work of Benjamin Franklin, he also suggests that people have come to think of their professional work and earning capital as almost a duty or 'a calling' and need it to feel content with professional life. This is furthered by the proposition that a man cannot engage in social action unless given a specialised task in an organisation.

On the other hand Weber (1930/2003) also says, "man does not wish to earn more and more money but will simply live as accustomed to live" (p. 60), with Tawney adding in the foreword, "labour is not merely an economic means: it is a spiritual 
end" (p. 3). This provides somewhat of an ambiguity in his work implying a responsibility beyond economic rationality and the pursuit of profit. Weber has been critiqued for this in the past by MacIntyre (1985) for appearing to select randomly from different disciplines of sociology, yet it is important to identify that he does not believe Protestantism fully caused the spirit linked to Capitalism, but considers it as one contributing factor. The full complexity of the topic and the limitations of his work are respectfully accepted.

\subsection{2: Bureaucratisation and the Exclusion of Feeling}

Weber also believes that bureaucratisation of the modern world and economic rationality in bureaucratic organisational forms has led to an exclusion of 'feeling' in tasks. Such depersonalisation is put down to capitalism in production and emotional detachment with work. Weber contends that because of bureaucratisation and rationalisation, this depersonalisation was an inescapable fate (Parker, 2002). However, as Parker explains, Weber seems to show some ambivalence towards bureaucracy. He does accept that there are advantages from a technical perspective, for example, precision and reduction of costs to name a few, yet the dehumanising outcome cannot be ignored.

"Bureaucracy develops the more perfectly, the more it is 'dehumanized', the more completely it succeeds in eliminating from official business love, hatred, and all purely personal, irrational, and emotional elements which escape calculation. This is appraised as its special virtue by capitalism”. (Weber, 1978, p. 973)

The following quote is an important acknowledgement for this research. The dehumanisation and redaction of feeling in business operations opposes the general characterisation of SMEs. SMEs are inherently personal and founded on incalculable emotional elements, as will be discussed in the following literature chapters. However, Weber's views on bureaucracy should be acknowledged in this case due to the lineage with means end rationality, a utilitarian perspective often adopted to make decisions in business.

Furthermore, despite believing that Weber identified a key trend in rationalisation 
and bureaucracy, MacIntyre (1985) critiqued him due to the wrong turn when he convinced himself that this trend was irresistible, implying that there is no alternative to economic rationality (Fevre, 2003, p. 14). Fevre (2003) further opposed Weber's view on economic rationality, as he believed morality was applied in the wrong way. This was in order to work harder to increase consumption, a thought that is materialistic and unrealistic due to capitalism. The final critique from Fevre refers to his work on bureaucracy, whereby there was an over emphasis on a means end search for efficiency though practical rationality.

As mentioned previously, when economic rationality is applied in the wrong place, there is often no room for morality. Establishing the role of moral perspectives is a key objective of this thesis. Consequently, the marginalisation and tensions between economic rationality and morality is central for discussion. Acknowledging the movement of sociology is also crucial for comprehension of CSR. For example, from sociology being colonised by economic rationality in the 1980's, to an emphasis on embeddedness and understanding that economic behaviour belongs to a social context following the 1990's. This misapplication of constructs and movement of perspective will be addressed in the following section when Durkheim's work is discussed.

\subsection{3: Morality}

Durkheim critiqued the fundamental ideas of individualism and utilitarianism that underpin economic rationality and Weber's theories. His contribution to the concept of economic rationality focused on morals more specifically, describing them as "the daily bread without which societies cannot exist" (Durkheim, 1893/1964, p. 51). His view was that "law and morality are the totality of ties which bind each of us to society" and "society is the necessary condition of the existence of morality" (pp. 398-399). This is very different to the views of Smith (1759/1976) who saw

morality "as natural, perhaps God-given, and not susceptible to fundamental change by any cause" (Fevre, 2003, pp. 6-7), yet Durkheim worked at showing that people could change morality and it wasn't god like. In his view it is a social construction, not a natural law. 
Durkheim's work was dominated by an attempt to understand industrial capitalism and the contention that work defines and gives moral meaning to our lives. $\mathrm{He}$ believed that industrial activities do have some purpose as they "respond to needs" (1964, p. 51), even if these are not moral. However, his main aim was to show how economic motivations were displacing morals, because morality was being subordinated to economic rationality. His view was that moral guidance would be driven out of industrial societies, largely due to this displacement and the reliance on economic rationality to create efficiencies. For example, as seen in Fevre (2003), Durkheim believed morals were the path to a good society, yet "the primacy of economic activity was responsible for the demoralization of society" (p. 4). This suggests that the social bonds that shape morality and created industrial capitalism could be the same that "weaken public morality" and kill it (Durkheim, 1893/1960, p. 4). Fevre concludes by suggesting that morality is increasingly likely to turn up as an instrument used to achieve economic ends, because demoralisation had proceeded so far (p. 7).

However, Durkheim also proposed that our occupational specialisation could provide a solution to the above issues and create a new morality (Fevre, 2003, p. 4). This is because it could provide a new type of solidarity, moderating egoism and emphasising a 'duty' to work. This is a similar concept to that of 'the calling' proposed by Weber. As seen in the quote below, specialisation could be a key part in recreating morality in society, although there would be a need for new rules to ensure conformity:

"Each individual will have the place he merits, will be rewarded as he deserves, where everybody, accordingly, will spontaneously work for the good of all and of each". (Durkheim, 1893/1964, p. 408)

This places an emphasis on his theme of collective consciousness, that opinion is one of collective collaboration, as highlighted in the quote below:

"A moral or juridical regulation essentially expresses, then, social needs that society alone can feel; it rests in a state of opinion, and all opinion is a collective thing, produced by collective collaboration". (Durkheim, 1893/1964, p. 5) 
Such specialisation can be compared with the contribution of Polanyi. He also critiqued Smith's (1776/1994) assumption that humans are naturally inclined to participate in trade and barter. The views of Polanyi and MacIntyre on economic rationality and their theoretical contribution will be further discussed in the following section.

\subsection{4: Embeddedness and Reciprocation}

Polanyi (1944/2001) can be described as a socialist and provides a unique contribution to the understanding of business and society. He implies that social class drove the market outcome of historical processes, not natural institutions arising from the characteristics of human nature. He stated that:

"Political and social impulse to tame markets had grown wherever markets had grown but in nineteenth-century Britain the disastrous experiment of the self-regulating market had been introduced". (Fevre, 2003, p. 23)

One of Polanyi's central concerns was establishing the natural behaviours that people may or may not be predisposed to. This shows lineage to Weber's discussions on the character of men and their disposition to engage in economically rational behaviours. Polanyi strongly critiques the assumption of the rational economic man, a concept constructed through the work of Adam Smith. He opposes the idea that man has a natural tendency to exchange and barter and maintains the fundamental point that economic activity is in fact embedded in society and social relations. This is similar to the beliefs of Durkheim on collective consciousness and strongly disputes the emphasis on individualism by Smith, focusing more specifically on the natural behaviors humans may be predisposed towards.

"The outstanding discovery of recent historical and anthropological research is that man's economy, as a rule, is submerged in his social relationships". (Polanyi, 1944/2001, p. 48)

"The term embeddedness expresses the idea that the economy is not autonomous, as it must be in economic theory, but subordinated to politics, religion and social relations". (Polanyi, 1944/2001, p. XXIV) 
The idea that there is a social context within economic behavior is expressed through Granovetter's (1985) idea of embeddedness. Fevre (2003) describes embeddedness as an excuse for sociology to invade economics, yet for Polanyi embeddedness is part of a critique of rational constructs.

Reciprocity and replication are also discussed within Polanyi's work. This is arguably a fundamental premise of CSR, how you would go about giving and if you expect anything tangible or intangible in return. The essence of reciprocation and civic virtue is evident in the following quote, "the performance of acts of exchange by way of free gifts which are expected to be reciprocated though not necessarily by the same individual" (Polanyi, 1944/2001, p. 49). This contribution resonates with the role of social capital (Putnam, 2000), providing sociological roots that anticipated the modern day interest in the concept.

It is clear to recognise the moral aspect of Polanyi's work and the influence this has on Macintyre's contribution. His critique of rational constructs and integrated view of the economy and society are transferred into MacIntyre's thinking. However, most prominent similarities according to McMylor refer to Polanyi's introduction of embeddedness of economic activity. The resemblance is identified when MacIntyre claims that virtues are "embodied in some shared account of our own context" (McMylor, 2003, p. 405), the start of his integrative explanation of economic activities.

\subsection{5: The Development of Moral Philosophy}

MacIntyre argues that every moral philosophy has some particular sociology as its counterpart, focusing specifically on moral positions within individual roles. His overarching theme is that of natural and artificial virtues and claims to have a 'general theory' appearing in his seminal work of After Virtue in 1981. This involved the main themes of virtues, goods, practices and institutions (Beadle \& Moore, 2006, p. 323), yet rejects the notion of human behavior as a science and believes that it in fact cannot be controlled. His view is that "virtue in the individual is nothing more or less than allowing the public good to provide the standard for individual behavior” (MacIntyre, 1985, pp. 236-237). 
MacIntyre can be seen as one of the first to have an impact on understanding morality in society and believed that problems and confusion occurred due to a reliance on reason as opposed to belief. He suggests that morality has lost all meaning, being "hollow - it's substance destroyed" (Fevre, 2000, p. 22) something he blames on Kant and his pursuit of a rational foundation for morality. The complexity of the individual and their morals expressed by MacIntyre can be seen in the quote below.

\begin{abstract}
"It was in the seventeenth and eighteenth centuries that morality came generally to be understood as offering a solution to the problems posed by human egoism and that the content of morality came to be largely equated with altruism. For it was in that same period that men came to be thought of as in some dangerous measure egoistic by nature; and it is only once we think of mankind as by nature dangerously egoistic that altruism becomes at once socially necessary and yet apparently impossible, and if and when it occurs, inexplicable”. (MacIntyre, 1985, pp. 228-229)
\end{abstract}

MacIntyre's work is similar to Polanyi due to the integrative approach to explain economic activities. For example, the quote below expresses his view of virtues and how they cannot be dealt with in isolation to their context. This is particularly forthcoming within the practice of CSR, as it too cannot be seen within isolation, be that practical or theoretical. This is because CSR takes inspiration from the realms of sociology, ethics and politics just to name a few. MacIntyre (1985) further proposes that "the catalogue of names does suggest how wide and heterogeneous the variety of moral sources is" (p. 10). This emphasises how diverse motivations for individual morality can be:

"...Any adequate account of the virtues in heroic society would be impossible which divorced them from their context in its social structure, just as no adequate account of the social structure of heroic society would be possible which did not include an account of the social structure of heroic virtues". (MacIntyre, 1985, p. 121)

Another philosophical theory covered by MacIntyre (1985) is emotivism that suggests, "all evaluative judgments and more specifically all moral judgments are nothing but expressions of preference, expressions of attitude or feeling, insofar as 
they are moral or evaluative in character" (p. 12). He argues persistently that we have too many rival moral concepts and no way of settling them rationally due to morals being judgments and "the language and practice of morality" being "in a state of grave disorder" (p. 256). He further quotes that "there seems to be no rational way of securing moral agreement in our culture" (p. 6).

MacIntyre (1985) does however appreciate that "particular judgments may of course unite moral and factual elements" (p. 12). This is again where his work relates widely to CSR. The construction of socially responsible behaviour may be based on an individual judgment, yet this too can involve both economic and socially constructed motivations to come to a final decision. Therefore, the key point is that these elements are not mutually exclusive and unite during the decision-making process of CSR.

One final idea from MacIntyre deals with honor. He suggests that to dishonor someone is to fail to recognise what is due. This is evident in his quote below.

"The concept of honor in Aristotle's world is very different to Goffman, honor is given to man what is due to him, to his kin, household and by reason of their having their due place in society". (MacIntyre, 1985, p. 116)

This shows lineage to Weber's concept of 'the calling' and can be linked to Polanyi's idea that if an individual has a place in society, they also have duties. Similarly, for a business to have a place in society, they also have duties to society. This is an argument for CSR in itself, in that if the business fails to recognise and act on societal duties, then it would be considered as a dishonor. The discussion regarding the moral standards of society is continued below, drawing from the views of Fevre (2000).

\subsection{6: Moral Standards of Society}

Ralph Fevre's book The Demoralization of Western Culture is one of social theory, looking at the relationship between the moral and economic. He attempts to address concerns of the moral standards of society and evaluates that a particular type of 
rationality, economic rationality, has significantly contributed to the demoralisation of western culture. Similar to MacIntyre (1985), he sees common sense as a subcategory of rationality and proposes that the problem of demoralisation is inherent in the way reason is applied. Consequently, the negative effects of rationality are where reason has been applied in the wrong place. This forms one of his main themes that economic rationality has ultimately become a counterfeit to morality, introducing the concept of 'ersatz morality' (Fevre, 2000, p. 209).

"We know economic rationality is in the wrong place when we feel morally compelled to do something. Economic rationality cannot legitimately found a morality and so what we are feeling is a sham, ersatz morality that we are better off without". (Fevre, 2000, p. 201)

Fevre further explains that due to capitalism, economic rationality has increasingly become a form of sense making. He proposes that in terms of our social constructions, we need to recognise and support the belief that economic rationality is in fact being applied in the wrong place and apply the different modes of sense making in the right place. This opposes Weber's view of economic rationality when morality is applied in order to work harder and increase consumption. The following quote explains the social construction and continual sensemaking during decisions:

"We have no absolute position, just a relative one that we must continually re-establish from moment to moment because the guidelines we followed when making last year's decision, even yesterday's decision, may not apply today or tomorrow". (Fevre, 2000, p. 8)

The implied relative position shows lineage to the unique and constantly changing environment that SMEs operate within. However, it is appreciated that economic rationality is still an important aspect of common sense; indicating that it simply took a wrong turn in the way that it was understood. As Fevre explains below, the current conception of economic rationality is outdated.

"Economic rationality is one of the most important reinventions of common sense in a more rigorous and robust form but it is based on an old common-sense idea: that everyone is out for themselves and interested in maximizing their own pleasure and minimizing their experience of pain". (Fevre, 2000, p. 200) 
Despite the idea being outdated, it is inferred above that it would be possible to update the conception of economic rationality by re-establishing the guidelines. As Fevre acknowledges, economic rationality is an important aspect of common sense, complementing MacIntyre's appreciation that moral and factual elements can unite when making judgments. Consequently, it is important that measures are taken to rectify the conception and wrong application in order to provoke a remoralisation of society.

\subsection{7: Summary}

To summarise, Weber was one of the first to bring attention to economic rationality, he considered economic factors as fundamental and to be considered above all during the explanation of decisions. His perspective centers on individualism, utilitarianism and the view that profit, production and efficiency are key to rationalisation, positioning it as an inescapable fate. His concept of 'the calling' further expands the conversation, suggesting that people work and earn money because they feel it is their duty and a prerequisite to feeling content. However, whilst Weber was an influential figure for economic rationality, Durkheim opposed his views. He suggested that bureaucratisation has led to a depersonalisation and lack of feeling in work, reducing moral consideration during decisions due to the overpowering nature of economic rationality.

The main theme drawn from Durkheim is that economic motivations have displaced morals because morality capitulated to economic rationality. Specialisation and division of labour were considered necessary for the cohesion of societies and as a way to create a new morality. This emphasis on the collective consciousness of society differs from individualism that is key to Weber's perspective. Collective consciousness strongly influences the relationship between business and society, particularly considering Durkheim's view that morality is a social construction that can be changed and molded. Specialisation can be compared with the contribution of Polanyi, with his assumption that humans are naturally inclined to participate in trade and barter. 
Polanyi's main argument is that economic activity is embedded in social relations and the collective consciousness of society, opposing individualism and implying decisions on CSR will be reflective of the societal context. He also touched on reciprocity and replication, resonating with one of the main questions of this thesis that asks if SMEs engage in CSR for reciprocal benefits, or for altruistic and ethical reasons. Polanyi shows similarities to MacIntyre due to the emphasis on morals, embeddedness and integration of economy and society.

MacIntyre's work focuses more specifically on natural and artificial virtues and the moral position of individuals. His contribution is important to emphasise that economic activities and virtues cannot be dealt with in isolation to their context. He rivals the view that any moral concept can be completely economic and proposes emotivism, whereby judgments are nothing but a feeling and therefore there is no way of settling rational agreement. MacIntyre also presents the topic of honor. If a business fails to recognise their duty towards society, then they are dishonoring their position, directly relating to the concept of CSR.

However, Fevre's critiques of Weber and Durkheim suggest economic rationality is in fact a counterfeit to morality when applied in the wrong place. His view is that economic rationality has increasingly become a form of sensemaking, resulting in the demoralisation of western culture. Morals are deemed the fundamental ingredient for a good society and therefore there is a need for sensemaking to be applied in the right place.

To conclude, having considered sociological theories that underpin CSR from the main perspectives of Weber, Durkheim, Polanyi and MacIntyre, the following section will focus on the role of ethical theories. Attention will be paid specifically to the theories commonly adopted to explain CSR, but also those proposed to be more suitable to explain the concept in SMEs.

\section{3: Ethical Theories}


The following section will bring ethical theories to the foreground of discussion, particularly those that have dominated the business and society literature to date. "Moral philosophy is an important aspect of business ethics" (Spence \& Rutherfoord, 2003, p. 3) and "ethical theory is the primary source of explanatory concepts" (Spence, 2014, p. 2014). With each theory there is a different way to assess ethics. Consequently, it is important to understand which theories frame CSR justifications and how they have developed throughout the years. It could be problematic to consider that there is one single theory to CSR (Brooks, 2008), but to establish if one is more relevant to different business contexts is merited. Table 2.1 presents an overview of ethical theories and adoption of these in research, specifically related to CSR in SMEs.

Table 2.1: Table of Ethical Theories and Relevant Literature (Adapted from Spence, 2014, pp. 378-379)

\begin{tabular}{|c|c|c|c|}
\hline Ethical Theory & Focal Point & $\begin{array}{l}\text { SME/entrepreneurship } \\
\text { Research }\end{array}$ & $\begin{array}{l}\text { Supporting } \\
\text { Research }\end{array}$ \\
\hline $\begin{array}{l}\text { Kantianism } \\
\text { (deontological) }\end{array}$ & $\begin{array}{l}\text { Do one's duty } \\
\text { according to } \\
\text { reasoned } \\
\text { consideration }\end{array}$ & $\begin{array}{l}\text { Anderson \& Smith (2007), } \\
\text { Clarke \& Holt (2010), } \\
\text { Lähdesmäki (2005) }\end{array}$ & $\begin{array}{l}\text { Kant (1781/1998), } \\
\text { Kant (1788/1997) }\end{array}$ \\
\hline Utilitarianism & Act according to & Ahmad \& Ramayah (2012), & Bentham (1789), \\
\hline
\end{tabular}




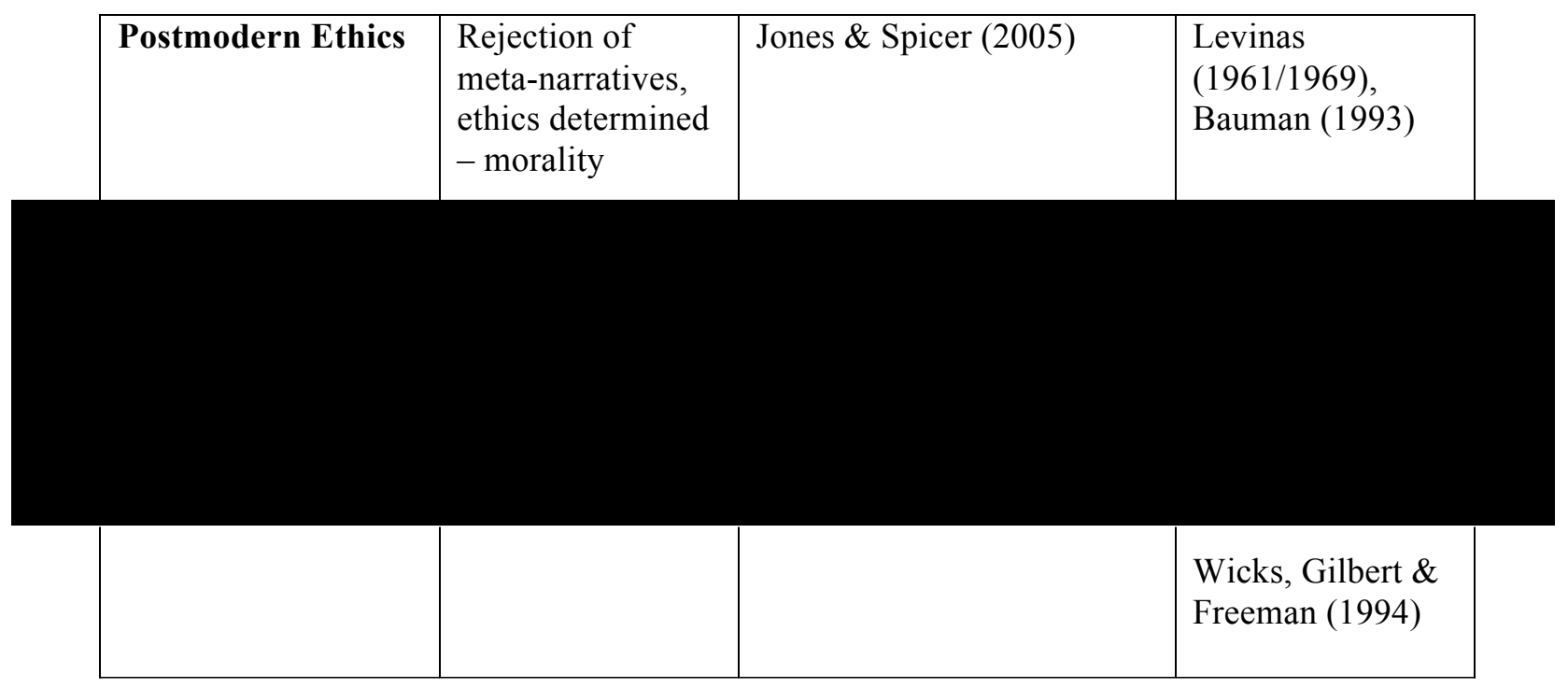

Note: * The works cited here are absolute classics and have been reprinted, translated and reproduced many times, lacking definitive versions. Therefore, they have not been included in the reference list. Works published since the beginning of the 20th century have however been included.

For clarity within this thesis, morality can be described as "the norms, values, and beliefs embedded in social processes which define right from wrong for an individual or community" and ethics as "the study of morality and the application of reason to elucidate specific rules and principles" (Crane \& Matten, 2016, p.8). Morality comes into play at all levels of analysis for CSR, with ethics and characteristics of the moral issue infusing every stage of decision-making. Consequently, establishing the different ways that ethics can be assessed is vital to develop understanding of this topic.

\subsection{1: Utilitarianism}

The CSR field is commonly associated with consequentialist ethics and a utilitarian position (Frederiksen, 2010), most notably linked to Bentham (1789/2007). This ethical perspective is based on the view that "an action is morally right if it results in the greatest amount of good for the greatest amount of people affected by the action" (Crane \& Matten, 2010, p. 100). Although, there are two elements of this ethical theory, act utilitarianism that focuses on individuals and determines the utility per act 
and rule utilitarianism that determines the overall utility of everyone following or rejecting one particular rule.

Utilitarianism has been applied to the management setting by the likes of Fritzsche and Becker (1984) and Premeaux (2004). Their empirical investigations concluded that managers make decisions based on utilitarian justifications when faced with ethical dilemmas. This way of thinking was also evident in the research of Besser, Miller and Perkins (2006) who considered how networked businesses impact community life for the greater good. Their findings suggest that business networks are a strategy for local economic development and those businesses embedded are usually better community citizens. This is closer to the small business literature due to their reliance on community embeddedness. However, their findings also suggest that social performance is engaged with as a proactive strategy to avoid discontent customers, following a similar large firm utilitarian perspective as seen in Arvidsson (2010).

In the SME and entrepreneurship setting specifically however, Ahmad \& Ramayah (2012) have also implied that consequences drive action, whereby it is the positive implications on performance that drive social responsibility in entrepreneurial ventures. Although their research context was in a developing country, this could explain the emphasis on performance. Lähdesmäki (2005) further researched the ethical nature of small business entrepreneurs. Whilst they did find that often it was a utilitarian kind of thinking adopted with regards to ethical reasoning, they also considered that this was not the sole theory evident. It was suggested that deontological and virtue ethics were also apparent and decisions were justified as moral based on different ethical validations, depending on situational factors.

Furthermore, there are criticisms of utilitarianism that should be acknowledged. For example, consideration is needed of the means taken to get to a particular endpoint, as MacIntyre (1985) claims, utilitarianism as a moral perspective constricts the vision of managers so that even ends are not reflected upon. Additionally, it implies that CSR is strategic and used as a way of maximising the final outcome of the decision to benefit the business. Frederiksen (2010) suggests that for large businesses CSR is not founded upon utilitarian assumptions, instead it is a 
deontological common sense morality that policies are based upon. He concludes that the ethical character of businesses do not adequately reflect the ethical foundation of managers. Consequently, it is apparent that there are moral considerations that need to be made for this ethical theory, with further exploration warranted.

\subsection{2: Egoism}

Ethical egoism can also be thought of as a consequentialist theory. This is most commonly associated with Adam Smith and concentrates more specifically on the individual (Reidenbach \& Robin, 1990). Smith deciphers that "an action is morally right if the decision-maker freely decides in order to pursue either their (short term) desires or their (long term) interests" (Crane \& Matten, 2010, p. 100). Whilst Smith is known for his economically rational position through the 'economic man', he did show some appreciation of others in The Theory of Moral Sentiments when he says:

"How selfish so ever man may be supposed, there are evidently some principles in his nature, which interest him in the fortune of others, and render their happiness necessary to him, though he derives nothing from it except the pleasure of seeing it". (Smith, 1759/1976, p. 9)

The above suggestion that people will help the fortune of others for the pleasure that they derive from it, may seem unusual from a man who defends the rational economic man. However, he justifies the claim by saying, "as we have no immediate experience of what other men feel, we can form no idea of the manner in which they are affected, but by conceiving what we ourselves should feel in the like situation" (Smith, 1759/1976, p. 9). This suggests that it is the imagining of oneself in a particular situation that elicits egotistic emotions that derive a response.

In the SME setting egoism is less commonly applied. However, Besser and Miller (2004) did find that businesses are driven by self-interest and variations of rationales when justifying social performance. Their research focuses on communities and employs sociological approaches, implying that an enlightened self-interest rationale strengthens networking and their support of communities. On the other hand, 
Longnecker, McKinney and Moore (1988) focus on the ethical position directing the behaviours of entrepreneurs. The findings suggest that rather than considering particular behaviours critically, entrepreneurs have a way of rationalising them. Concerns are raised about the implications of increased entrepreneurialism in society when such ethical egoism dominates entrepreneurs' behaviour.

Smith's perspective of the rational economic man has set the foundations of economic rationality. Both utilitarianism and egoism can be related to the strategic aspect of CSR whereby ends justify the means. This concept can be presented via Smith's term the 'invisible hand' (Reidenbach \& Robin, 1990), an idea that there are unintentional social benefits for those that follow self-interested actions. This position has been popular for management decision-making in the past (Lähdesmäki, 2005). However, despite consequentialist theories being popular to date in large businesses (Frederiksen, 2010; Premeaux, 2004), there are a number of criticisms. As Spence (2014) suggests, arguments for consequentialist ethics are more appropriate on the level of large scale or public decision-making, suggesting that there are more appropriate ethical theories to explain CSR in SMEs.

\subsection{3: Kantianism}

An alternative ethical framework is deontological ethics, most commonly associated with the seminal work of Immanuel Kant (1781/1998). Deontological ethics are duty based and concerned with rule following and individuals doing the right thing, not just for the consequences of an action. When applied to CSR, alternative to the consequentialist roots in economic rationality, it could be considered that deontological ethics underlie the ethical position of CSR because of altruism and philanthropy. In comparison to strategic motivations for CSR, this perspective is based on morals towards society and a concern for stakeholders. The notion of CSR as a "multi-fiduciary activity" (Brooks, 2008, p. 64) takes into account stakeholder interests during decision-making rather than only looking at the end result.

Anderson and Smith (2007) adopted Kantianism in the entrepreneurial setting. They suggest that there is a moral imperative in entrepreneurship and found that societal values provide the benchmark that is employed in the social legitimisation process. 
However, simply behaving entrepreneurially was not enough to authenticate actions, the acceptance of such values was context dependent and only authenticated if congruent with socially approved behaviour. Moral evaluations were therefore more concerned with societal values and benefits. Clarke and Holt (2010) also considered Kantian ethics, but from a reflective judgement perspective that identifies judgement as social performance. Their findings contend that ethics is involved in the entrepreneurial creation of value as it the individual responsible for creating it, not the business. Therefore, it is not a case of judging value from a utilitarian perspective, but from the individual rules created via personal experience.

\subsection{4: Additional Ethical Theories}

Whilst consequentialist and deontological theories appear to be the most commonly accepted perspectives to assess ethics in the CSR field, past literature has adopted a multitude of moral frameworks. Frederiksen (2010) provides a brief overview of these in his paper concerning CSR and philosophical moral theories. However, it is not within the scope of this thesis to discuss them all in detail and therefore the remaining theories identified in his overview and Table 2.1 will be acknowledged briefly. These other approaches include virtue ethics, social contract theory, discourse ethics and postmodern ethics.

Virtue ethics is one of the theories that has previously been used to assess CSR in the SME setting. It originates from Aristotle and has been adopted by Blackburn and McGhee (2007) and Dunham (2010) to consider the character of the individual. In contrast to Kantianism, practical wisdom, personal values and the purpose of the entrepreneur are considered important to look beyond economic rationality (Spence, 2014). Whilst this does resonate with the SME setting whereby the ownermanager's character can be assessed, it fails to take into account the context of the business. Again, this warrants exploration of ethical theories that are more suitable to account for contextual factors.

Further theories adopted in the literature include social contract theory that has been utilised by Bucar, Glas and Hisrich (2003) and Koos (2012) who focus on rules that manage behaviour. Discourse theory was also used by Lähdesmäki (2012) who 
takes into account the discursive resources that legitimise entrepreneurs' sense of self when talking about CSR and finally postmodern ethics has been used by Jones and Spicer (2005) and is defined by uncertainty, doubt and spontaneous action in response to feeling the need to act. Whilst these theories were not looked at in detail, they are important in order to set the context for newer moral approaches being explored.

The above review shows that currently there is not one ethical theory that is adequate to explain CSR in the SME setting. This makes apparent the need for renewed theoretical perspectives. Past literature has identified two moral perspectives that could potentially be of value to reflect the composition of CSR in SMEs; these include the ethic of care and moral proximity (Spence, 2014). Whilst these theories have gained traction in the business ethics field, they have been marginalised by the ethical theories previously mentioned. These moral perspectives will be a key focus of this thesis and will be discussed in more detail in the following section.

\subsection{5: Ethic of Care}

In recent publications, Spence $(2014,2016)$ uses the standpoint of Freeman and Liedtka (1991) as a starting point for theoretical developments. One of their main arguments, and the view that Spence draws from is that:

"Corporations are places in which both individual human beings and human communities engage in caring activities that are aimed at mutual support and unparalleled human achievement... This proposition pushes us beyond the language of rights and responsibilities to a focus on the ethic of care, which recognizes needs and affirms the self and its linkage with others". (Freeman \& Liedtka, 1991, p. 96)

The position that was proposed directed Spence towards the value and appropriateness of the feminist perspective to advance theoretical developments for CSR, particularly the ethic of care. Whilst the ethic of care is informed by feminist ethics, these terms are not interchangeable and can be differentiated, as pointed out by Borgerson (2007). However, Spence's (2016) understanding that "feminist ethics is concerned with contextualised reflections on responsibility, relationships, and 
experience and tends to value connections over autonomy" (p. 3) emphasises the relevance to the SME settings. A brief summary of underlying literature relating to the development of the ethic of care and CSR theory is presented below.

Carol Gilligan (1982) first sparked debate about the 'different voice' of women in her book In a Different Voice. She proposed that psychologists ignored that women hold a different voice to men during questions of moral reasoning (Kohlberg, 1984). Gilligan, as a student of Kohlberg had "...taken issue with the use of justice as the pre-eminent determinant of moral reasoning" (Fisher \& Lovell, 2009, p. 107). She proposed two value systems; ethic of justice and ethic of care and recommended that empirically, research suggests that men are more aligned to individual rights and women to the responsibility of others, although not restricting them to these categories. Virginia Held (2006) took the ethic of care and expanded Gilligan's ideas by identifying five assumptions outlined in Table 2.2 .

\section{Table 2.2: Five Assumptions of The Ethic of Care}

\begin{tabular}{|l|l|}
\hline 1. & Meeting the needs of others for whom we take responsibility \\
\hline 2. & Valuing emotions (for example, sympathy, empathy, sensitivity) \\
\hline 3. & Accepting partiality \\
\hline 4. & The private sphere as a territory for morality \\
\hline 5. & People are relational and interdependent \\
\hline
\end{tabular}

Held (2006) argued that the domain of ethics is dominated by theories that advocate a rational approach. She suggests that the ethic of care is more appropriate than other theories such as Kantianism or utilitarianism as it acknowledges the limitation of markets for social decisions. Held further advocated context specific approaches to moral theory, noting that currently there is a "problematic reliance" (Spence, 2014, p. 383) on abstract rules. The contextual approach supported by Held is not accepting of the distinction between public and private, implying that personal and business spheres are merged.

Spence (2016) drew from the ideas of Held at this point when she recognised corresponding features of CSR in SMEs. She used the alignment of broad characteristics to justify the ethic of care as a suitable theoretical lens to expand the 
debate in this research area. As a theory, it does not focus on principles, outcomes or processes from traditional theories, but relationships and context. The main alignments Spence drew from the assumptions of Held (2006) are displayed in Table 2.3. The ethic of care feature is outlined alongside the corresponding characteristic of CSR in SMEs. The background empirical research that identified these characteristics will be acknowledged in the following chapter.

Table 2.3: Corresponding Features of The Ethic of Care and CSR in SMEs as adapted from Held (2006) and Spence (2016)

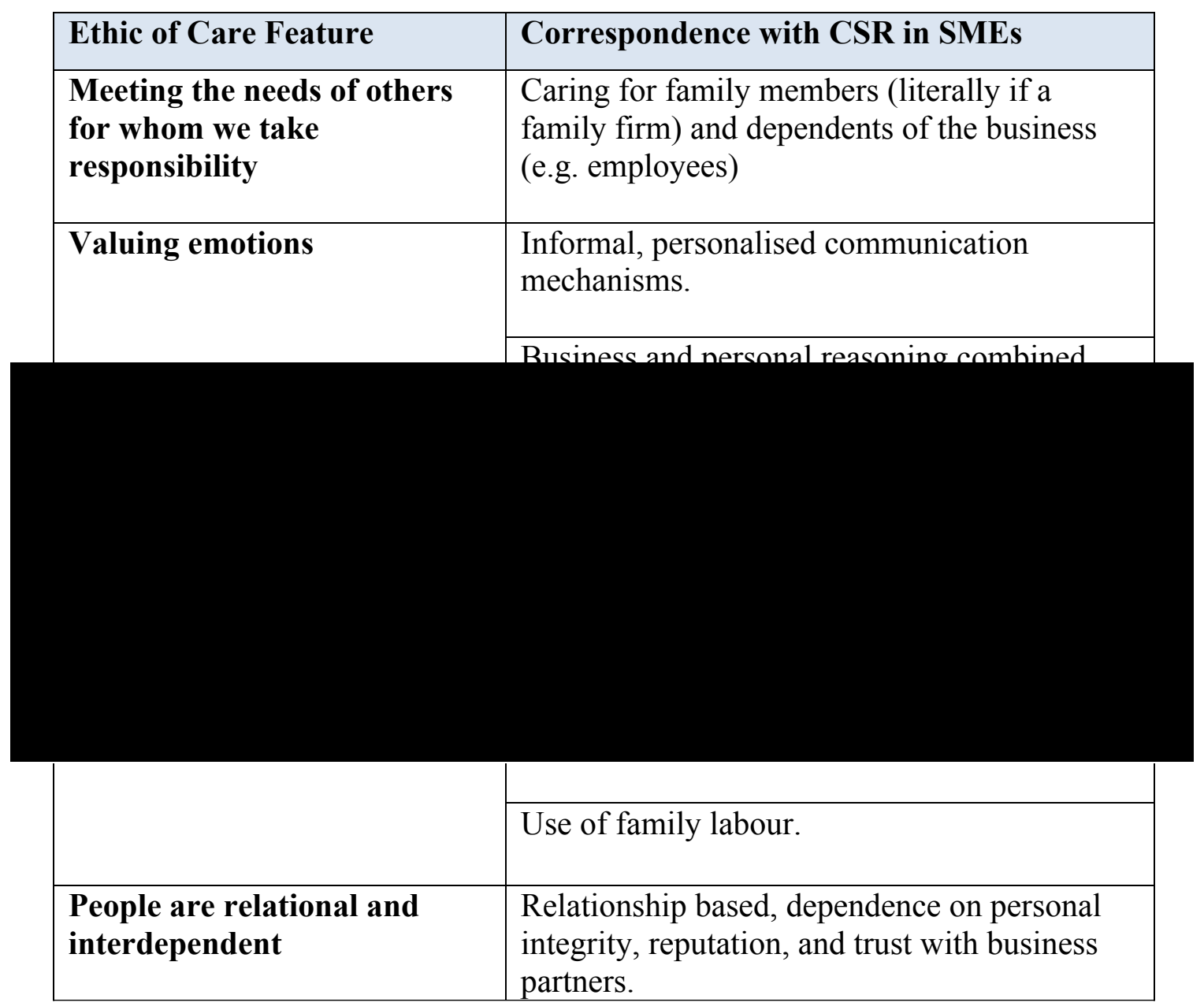

In the literature, Bauman (2011) has found that the ethic of care is appropriate in the large firm during crisis situations. Findings suggested that it is most effective in comparison to virtue ethics and ethics of justice when it comes to stakeholder concerns. Von Weltzien Høivik and Melé (2009) also considered the ethic of care to reflect the empirical evaluations of ethics in SMEs. They observed that a globally 
embedded SME engages in a number of CSR initiatives and exerts influence on their supply chain, concluding that this theory and concern for certain aspects of the common good are important aspects of corporate global citizenship.

However, it is Spence $(2014 ; 2016)$ who has strongly advocated the ethic of care as a relevant avenue to expand the CSR and entrepreneurship literature in SMEs, with a recent call for the research agenda to incorporate this theory. Her paper Small Business Social Responsibility: Expanding Core Theory redraws two core CSR theories with this perspective, these being stakeholder theory most commonly associated with Freeman (1984) and Carroll's (1991) CSR pyramid. There have been little theoretical developments for CSR in SMEs so this was a welcome development. Spence (2014) offers the ethic of care as one of the two proposed theoretical avenues for size sensitive ethics, alongside moral proximity. It is the development of this discourse and evaluation of the two moral perspectives that this thesis will make a contribution.

\subsection{6: Moral Proximity}

Moral proximity is the second perspective Spence proposed to assist understanding of the social and ethical components of SMEs. The concept draws from the moral intensity literature, first introduced by Jones (1991) through an issue-contingent model of ethical decision-making (Spence, 2016, p. 381). Jones proposes that every ethical issue can be represented in terms of moral intensity, whereby it "refers to characteristics of the ethical issue that compel the decision maker to employ ethical reasoning" (McMahon \& Harvey, 2007, p. 337). Ethical decision-making is a key aspect of CSR in the SME setting and has been a consistent theme in the literature. It is adopted as an appropriate central theme to this thesis.

Jones' framework can be used to evaluate different ethical situations and suggests that, "characteristics of the moral issue itself infuse every stage of decision-making" (Spence, 2014, p. 381). The framework consists of six constructs inclusive of moral

proximity (see Table 2.4). According to Spence (2014), it is 'proximity' and 'concentration of effect' that are most relevant for SMEs. This is due to distance being "the manner in which human beings view moral issues in respect of physical, 
psychological, cultural and social parameters" (p. 382). These parameters are what links responsibility to the owner-managers and those closest to them physically and emotionally (Jaffe \& Pasternak, 2006).

Table 2.4: Jones' (1991) Constructs of Moral Intensity in Ethical DecisionMaking

\begin{tabular}{|l|l|}
\hline 1. & Proximity, the feeling of social nearness to the beneficiaries/victims of an act \\
\hline 2. & Magnitude of consequences \\
\hline 3. & Social consensus that an act is moral \\
\hline 4. & Probability of an act occurring and associated effect/harm \\
\hline 5. & Temporal immediacy of an act and the effects being felt \\
\hline 6. & $\begin{array}{l}\text { Concentration of effect is an inverse function of the number of people } \\
\text { impacted by an act of a certain magnitude }\end{array}$ \\
\hline
\end{tabular}

Accordingly, the proximity construct is comprised of four types of closeness: physical, psychological, cultural and social. Physical proximity can be described as the "spatial closeness between a decision-maker and those affected by the moral act" (Mencl \& May, 2009, p. 206) and is the only type of proximity not implied on a personal level. Social proximity regards the identification as a group member dependent on feelings of belonging and similarity (Lähdesmäki \& Suutari, 2012). Psychological proximity focuses on a high commitment to others and refers to an affective closeness; an example would be towards family or friends (Mencl \& May, 2009; Wildermuth, De Mello e Souza \& Kozitza, 2017). Finally, cultural proximity regards a feeling of closeness to those from the same or similar culture.

These classifications are all intertwined and collectively influence the implicit orientations of SMEs owner-managers when making decisions (Spence, 2014). For example, as Mencl and May (2009) consider, there is a "theoretical overlap" (p. 206) between cultural proximity and the additional dimensions. Therefore, in their research they do not consider it as a mutually inclusive category, rather integrated within the others. Lähdesmäki and Suutari (2012) further explain that the discussion regarding the dimensions of proximity collectively addresses the context of a firm in relation to its stakeholders, revealing context specific implications for CSR engagement. This is an element lacking in previous theories. 
According to Jones (1991), proximity influences a moral agent's perception of the "moral imperative" (p. 327) or salience of the moral issue. Research in the past has therefore explored stages of the ethical decision-making process and proximity (McMahon \& Harvey, 2007; Mencl \& May, 2009; Ryan, David, \& Reynolds, 2004; Singer, 1998). The most recent of these is Wildermuth et al. (2017) who found that psychological proximity, as proxied by empathy and friendship, impacts moral reasoning in corporate human resource managers. This is whereby care is strengthened with closeness. From this perspective, proximity could be considered as a key determinant of ethical decisions and behaviour.

However, research extends beyond decision-making of the individual, exploring the separate classifications of proximity and their influence on business operations. The implications of physical closeness for SMEs are represented in the embeddedness (Granovetter, 1985) and community literature (Besser \& Miller, 2001; 2004; Besser et al., 2006; Campin, Barraket \& Luke, 2013). However, more recently other forms of proximity haven taken prominence. For example, Lähdesmäki, Siltaoja and Spence (2017) found that social proximity is more important for stakeholder salience than geographical, whereby the emphasis is not physically being next to each other but actually "having a common purpose and perspective that one might expect to develop in the small firm" (Spence, 2014, p. 382).

Currently there is dominance on specific quantitative enquiry when investigating the role of moral proximity; this is due to the search for causes of behaviour in the decision-making process of individuals. However, the above implies that there is a need for more research that considers the interaction between classifications of proximity on business operations. McMahon and Harvey (2007) did make a call for alternative, in-depth exploration of moral intensity. They believed that this would reveal more accurate results of the influence of proximity on decisions. Furthermore, whilst Lähdesmäki and Suutari (2012) consider social proximity and CSR in small business qualitatively, there is still very little work on the broader concept of moral proximity and the applicability to SME's involvement in CSR. Therefore, further research is warranted in this context, specifically with a qualitative approach. 
As Spence (2014) explains, "moral proximity provides a space-based analysis of the ethical and social orientation of small firms" (p. 382). This acknowledges the context of the SME. On the other hand, the moral lens of the ethic of care differs by explaining the behaviours and practises resulting from proximity. These moral lenses are claimed to be central for theoretical developments of CSR in SMEs.

\subsection{7: Summary}

In summary, the aim of this chapter was to establish the relevance of different ethical theories that frame CSR justifications in SMEs. It is consequentialist and deontological theories that have most commonly been adopted in CSR literature. For utilitarianism, an act is morally right when consequences drive the action. Therefore, when owner-managers pursue CSR for profit implications, the act is still justified as moral as their focus is on the outcome. Alternatively, egoism contends that a business is behaving ethically when driven by self-interest, focusing on the individual more specifically. Actions are therefore rationalised as moral when pursuing short-term interests or long-term goals.

Utilitarian ethics are often associated with large firm research because of their power and magnitude of consequences. However, they lack the specificity that is needed for the SME context and are ignorant of the moral considerations for achieving the end result. This ignorance can constrict owner-managers' vision so that even ends may not be reflected on. For egoism, focus on the individual is more in keeping with ethical decision-making in SMEs because of the integral role of the owner-manager. However, it is less commonly applied in the CSR research. The reasoning for this could be because behaviours are rationalised, rather than critically considered. The worry is that with a rise of entrepreneurs and SMEs, this could have negative implications on society. These consequentialist theories can be associated with the strategic view of CSR, implying that it is founded as an instrumental and economically rational construct.

Alternatively, deontological ethics are duty based and concerned with universal rule following. This means treating others as one would expect to be treated, not as a means to an end. Kant's perspective provides an alternative to Bentham and Mill 
and could be seen to underpin the morally rooted position of CSR, whereby decisions take the concerns of stakeholders into consideration. It is societal values that provide the benchmark for ethical standards in this perspective. Although, this view has been critiqued, primarily because universal standards are not considered enough to legitimise actions. Instead, it is proposed that rules are created based on personal experience and actions legitimised via the congruence of values within a specific context. These are elements that this ethical theory fails to take into consideration.

Additional theories such as social contract theory, discourse theory, postmodern ethics and virtue ethics have been considered briefly to establish an overview of the literature. Virtue ethics in particular have been utilised to assess CSR in SMEs, focusing on the character of the individual, similar to egoism. However, this theory differs in that it acknowledges the importance of practical wisdom and personal values to look beyond the confinements of economic rationality. That being said, as a theoretical frame it still fails to take into account the context of SMEs. It could be recognised therefore that there is not yet an ethical theory that adequately acknowledges the role of the individual and context, suitable for the SME setting.

More recently, it has been argued that the domain of ethics is dominated by theories supporting the rational approach, revealing a problematic reliance on abstract rules. Consequently, there has been a rise in interest for previously marginalised, or new theories to assess ethics and a call for size-sensitive theories to be incorporated into the research agenda. Moral proximity has been identified as a moral lens that does acknowledge context, an aspect identified as missing in dominant theories. This perspective provides an analysis acknowledging the influence of physical, social, psychological and cultural proximity on moral reasoning, parameters that link responsibility of the owner-manager to stakeholders.

Corresponding features of CSR in SMEs were also identified in the ethic of care, whereby the focus is on relationships and context. Key characteristics of this perspective include the acceptance of merged spheres, partiality and role of emotions in decision-making. As an ethical theory, the ethic of care differs by explaining the behaviours and practises resulting from different classifications of proximity. Both 
of these moral lenses are claimed to be central to theoretically progress the CSR debate in SMEs and it is to this development of the discourse that the following research will focus on.

\section{4: Chapter Conclusion}

This chapter provided an insight into the foundations of CSR by exploring the development of economic rationality and morality as sociological constructs and establishing the role of ethical theories. The literature rivals the view that any moral concept can be completely economic and suggests that over time, economic rationality has been applied in the wrong place, displacing morals. This identifies a need for morality to be applied in the right place, a key focus for this research. An evaluation of ethical theories also uncovered a need for theoretical perspectives more relevant to frame CSR in the SME setting. Current theories do not adequately account for context and lack specificity needed for the SME setting. This prompted research into previously marginalised and new theories to address this gap in the following study. The next chapter will now turn to the CSR literature. Key themes and developments will be acknowledged to show how the CSR concept has progressed over the years. Following this, attention will turn to CSR literature in the specific context of SMEs, the key research area of this thesis. 
Figure 3.1: Chronology of CSR developments (adapted from Ibrahim, 2014)

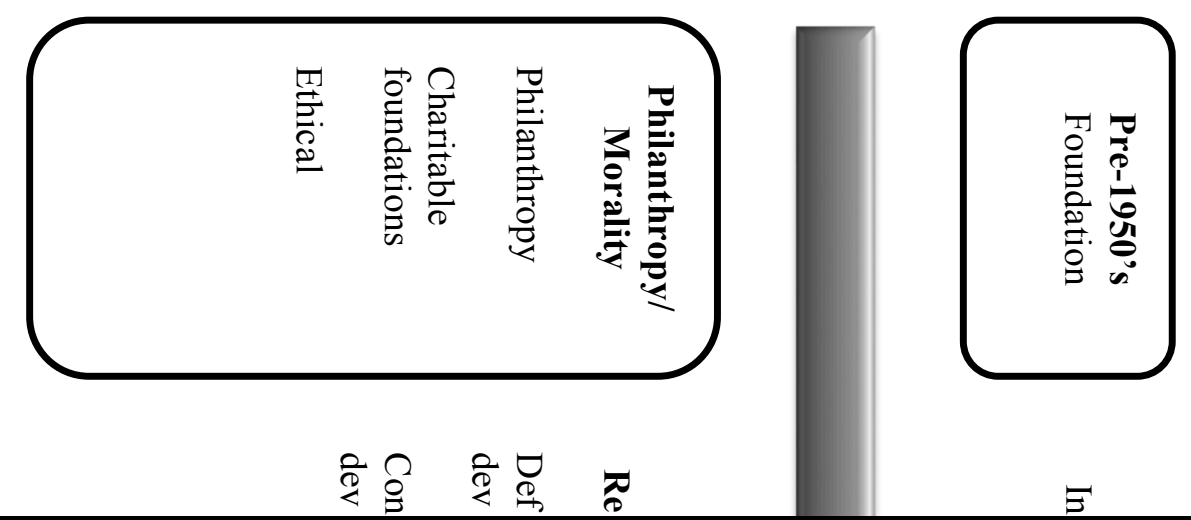

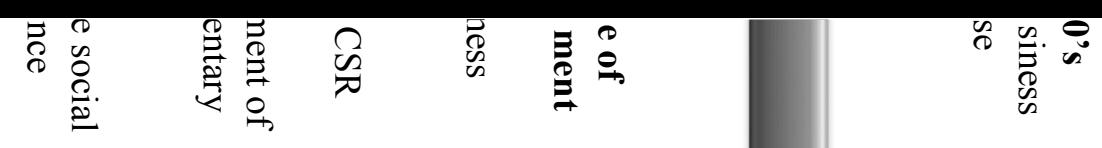

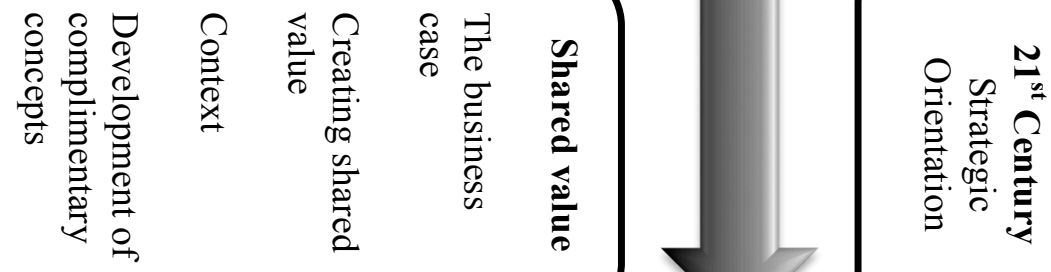




\section{Chapter Three: Literature Review - Corporate Social Responsibility}

\section{1: Introduction}

The following chapter provides a review of existing literature to identify gaps in the CSR discourse. The chapter is split into two main sections and begins with an analysis of the chronological development of CSR (section 3.2). This defines key themes and areas of study that have advanced the CSR debate, with insight into the shift from a purely philanthropic concept to strategic orientation. One gap in the literature is the lack of SME specific research. Due to the significant contribution of SMEs in the UK context, this prompted exploration of the small business conception of CSR. The second section (section 3.3) therefore has a more specific focus on the SME setting, as the main context for this research. Focus turns to defining the CSR concept for SMEs (section 3.3.2 and 3.3.3) and establishing distinct size characteristics. Section 3.3.4 furthers this by reflecting on the current position of the research agenda for SMEs. Following this, the theoretical (section 3.3.5) and analytical perspectives are defined (section 3.3.6) and the chapter summarised.

\section{2: CSR literature}

This section starts with an initial overview of relevant CSR literature. Social responsibility in SMEs is still a comparatively under-researched aspect of CSR and therefore an overview is necessary to establish the research context. Due to the expanse of literature emerging from a range of contradictory and complimentary disciplines, a complete review is not possible or necessary. Therefore, key thematic developments of the literature will be focused on and avenues for future research established to progress understanding of CSR.

\subsection{1: The Evolution of CSR}

The notion that organisations have an impact on the wider community is not new. Charitable principles and concern for society can be traced back for centuries 
(Carroll, 1999; Eberstadt, 1973) and "the philosophies of corporate and individual philanthropy predate the current interest in CSR by hundreds of years" (Brooks, 2005, p. 402). However, despite the long history, CSR is still a nebulous concept (Davis, 1960) with 'unclear boundaries and debatable legitimacy' (Lantos, 2001, p. 595). To this day, there is still not one universally accepted definition, although developments of a formal nature have been considerable in the $20^{\text {th }}$ and $21^{\text {st }}$ century (Carroll, 1991). Considering the depth and variability of CSR as a concept, it is wise to focus on the past eighty years where there has been a huge proliferation of knowledge on theory, research and practice.

It was following the Wall Street Crash of 1929 that steps were made to gain a better understanding of social responsibilities in business. Berle and Means (1932) believed that because of the separation of ownership and control in the modern business world, the management of large organisations owed responsibility to investors, to be achieved through increased transparency and accountability as an embedded voting right. Barnard (1938) also advocated a concern for society and outlined the moral considerations of a business in his book The Functions of the Executive, viewing the business as a co-operative system. This work was closely followed by that of John Maurice Clark's (1939) Social Control of Business and Theodore Kreps' (1940) Measurement of the Social Performance of Business. These initial acknowledgements of US business responsibility were seen as the starting point of a true academic interest in social responsibility.

\subsection{2: Defining Responsibility (1950-1960's)}

It was the 50's and 60's that triggered a newfound understanding of social responsibility, with steps taken to establish a more comprehensive definition and understanding of the concept. This period was described as "the modern era" of CSR (Carroll, 1999, p. 268), with prominent contributions coming from the likes of Bowen (1953), Levitt (1958) and Davis (1960). Each of these acknowledged that business responsibility expands beyond just economic interests, with Bowen (1953) being labelled the "father of corporate social responsibility" (Carroll, 1999, p. 270) following his seminal book, Social Responsibility of the Businesses, what is thought to be the first use of the exact term 'corporate social responsibility'. 
Bowen (1953) was concerned with the impact large organisations have on society as centers of power and showed an appreciation to citizens who are affected in any way by business decisions and actions. In this case, social responsibility is seen as a voluntary act in addition to normal business, which is driven by the expectations of society (Windsor, 2001). Bowen (1953) defines CSR as "the obligations of businessmen to pursue those policies, to make those decisions or to follow those lines of action which are desirable in terms of the objectives and values of our society" (p. 6). This will act as the starting point for the discussion on the development CSR as a construct.

Davis (1960) had similar views to Bowen (1953), only differing with his opinion that economic functions are the primary concern of business. His main theme was the balance of power and responsibility for the businessman when their actions impact the community, whereby "social responsibilities of businessmen need to be commensurate with their social power" (p. 71). Davis further proposed the "iron law of responsibility', suggesting that if this power is neglected, it will result in an erosion of overall business power. His final contribution was that social responsibility is likely to bring long-term economic gain, a commonly accepted proposition and initiation of the business case debate.

Despite influential developments of CSR from the likes of Davis (1960), the concept still remained contested, with Levitt closing the 1950's by warning people about the dangers of social responsibility. His main contention, similar to the thoughts of Friedman (1970), was that long-run profit maximisation should be the dominant objective of business practice. He described social responsibilities as "corrosive distractions" (Levitt, 1958, p. 49) and implied that being socially responsible is not the job of businesses, pleading for them to let government take care of the general welfare of society. However, even with the prominent economic standpoint of Levitt (1958), he still recognises that "it often pays not to squeeze the last dollar out of the market - especially when good will is a factor in the long term outlook" (p. 49).

\subsection{3: The Scope of CSR (1970's)}

The 1970's saw a huge increase in the popularity of CSR and the shaping of a new social consciousness. This awareness was stimulated by Milton Friedman's famous 
publication The Social Responsibility of the Business is to Increase Its Profits, which took the shareholder or neo-classical perspective that the sole responsibility of the business is to generate profit. Following this publication, the academic debate shifted to try and find a link between CSR and good business performance, in order to prove that social concerns do not distract from fulfilling the primary responsibility towards shareholders.

This resulted in a number of main themes emerging in the literature, inclusive of the changing social consciousness and awareness of social responsibilities, CSR definition and scope, corporate social responsiveness and corporate social performance (CSP) (Crane, McWilliams, Matten, Moon, \& Siegel, 2008, p. 31). Alongside these areas of focus, there was also a large academic contribution that was gradually being developed empirically through business practices.

In one of the first CSR books of this time frame, Johnson (1971) considered four definitional viewpoints to analyse the concept of CSR. These viewpoints were inclusive of: conventional wisdom, long-run profit maximisation, utility maximisation and lexicographic utility theory. Conventional wisdom defines a socially responsible firm as "one whose managerial staff balances a multiplicity of interests. Instead of striving only for larger profits for its stockholders, a responsible enterprise also takes into account employees, suppliers, dealers, local communities, and the nations" (Johnson, 1971, p. 50). This definition implies the infancy of the stakeholder theory because of the 'multiplicity of interests' and specific special interest groups named. It could therefore be considered as a "precursor of the stakeholder approach" (Carroll, 2008, p. 29).

The second long-run profit maximisation perspective suggests that "businesses carry out social programs to add profits to their organization" (Johnson, 1971, p. 54), implying similar views to Davis (1960) and referring to what would later be coined the 'business case'. Johnson (1971) continued to describe utility maximisation, whereby businesses "seek multiple goals rather than only maximum profits" (p. 59) and lexicographic utility theory whereby targets and goals of the business are shaped by past experience of their business and other similar businesses. It was concluded that whilst these definitions may seem contradictory, they are "complementary ways of viewing the same reality" (p. 77). They also account for the shift in perspectives 
of this decade.

Another prominent contribution for CSR definitional developments, as identified by Carroll (1999), was the definition from the Committee of Economic Development (CED). The definition followed observations that the relationship between business and society was changing substantially. They noted that business was expected to resume larger social responsibilities as "business functions by public consent" and "its basic purpose is to serve constructively the needs of society" (Committee for Economic Development, 1971, p. 11). The CED contributed an important practitioner point of view to defining social responsibility through a three concentriccircled definition as seem below.

Figure 3.2: CED model of CSR (Sen, 2011, p. 19)

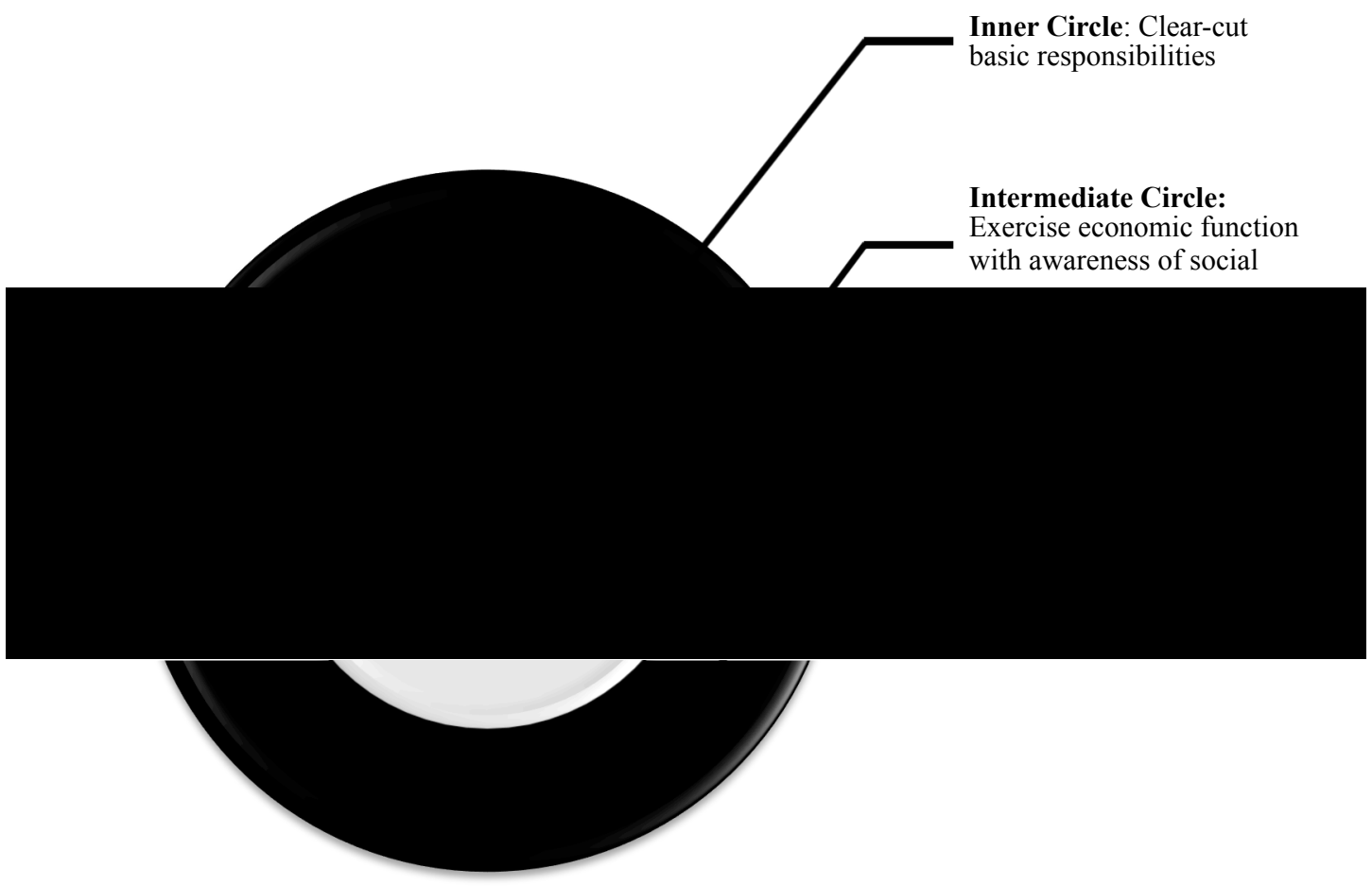

With regards to the shift in social consciousness, it could be seen as a response to the late 1960's social movement with regards to issues of worker safety, the environment and responsibilities to employees. These topics were at a point of transition from matters of special interest to formal government regulations, following the view of Levitt (1958). However, the constricted economic perspective was now being challenged. Whilst Steiner (1971) maintained Friedman's (1970) economic view, social responsibilities were being acknowledged. He aligned with 
Davis (1960) suggesting that responsibility should be proportionate to size and was one of the first to consider the influence of individual decision-making for social engagement with stakeholders.

Businesses were now expected to both meet the expectations of shareholders and civil society, in addition to promoting desired changes in society, what was termed 'social responsiveness' by Sethi (1979). Corporate social responsiveness was a dimension of corporate behaviour identified by Sethi, alongside social obligation and social responsibility. It is characterised as anticipatory and can be described as corporate behaviour adapting to social needs. These developments feed into the CSP debate that developed in the 1980's. At this point CSR was seen as responsive, with the main principle being charitable or philanthropic in order to support societal causes and positive change. However, during this period there was no perceived link between CSR and tangible benefits such as performance or profits. Consequently, CSR was driven by the needs of society as a reactive action.

To briefly summarise, it is obvious there are mixed views on the scope and legitimacy of CSR. The likes of Levitt (1958), Carr (1968) and most notably Friedman (1970) argue that CSR is not a legitimate practice for businesses. However, others such as Berle and Means (1932), Barnard (1938), Kreps (1940) and later Bowen (1953), Davis (1960) and Carroll (1979) support the view that business has a broader responsibility than profit maximisation for shareholders, supporting the CSR notion. Either way, as Carroll predicted, increased awareness of CSR meant that it was a subject of considerable interest moving forward.

\subsection{4: CSR Boundaries and Implications (1980's)}

Discussions of the 1980's shifted focus from the scope of CSR to the boundaries. This shift was intensified by the political and economic changes of this time, whereby greater emphasis was placed on individual and business responsibility as opposed to governmental. Deregulation, privatisation and the questionable practices of multinational companies (MNCs) brought social responsibility to the fore and revealed a disregard of stakeholders (Windsor, 2006). This prompted the focus on stakeholder theory and the questioning of whom a business should be responsible 
for. There were also numerous and significant developments for the CSP debate, whereby the tangible benefits of CSR were queried (Frederick, 2006).

Additional to the previously mentioned definitional developments, it was Carroll (1979) who provided the largest contribution in this era to define the scope and domains of CSR. His beliefs are similar to Swart (1976) by suggesting that, "in a complex and interdependent world there is no corporate environment per se divorced from society" (p. 322), what does exist is a corporate-social environment. Carroll (1979) builds CSR as a construct encompassing "the economic, legal, ethical, and discretionary expectations that society has of organisations at a given point in time" (p. 500) with tentative support for these weightings of the four components (Aupperle, 1984; Aupperle, Carroll \& Hatfield, 1985).

The economic domain refers to the business having a responsibility to produce and sell goods and services that adhere to consumers, whilst still being profitable. The legal domain reflects the expectation to adhere to the law at all times. The ethical domain reflects the need to meet society's unwritten rules and expectations of business behaviour and finally, the philanthropic domain refers to the voluntary responsibilities that go beyond ethical expectations. For CSR, it is proposed that these responsibility domains need to be simultaneously fulfilled. Carroll's (1979) four-part definition was depicted as a pyramid, as is seen in Figure 3.3 below and has acted as the leading framework in the literature with "enduring popularity" (Burton \& Goldsby, 2009). 


\section{Figure 3.3: Carroll's (1979) Pyramid of CSR}

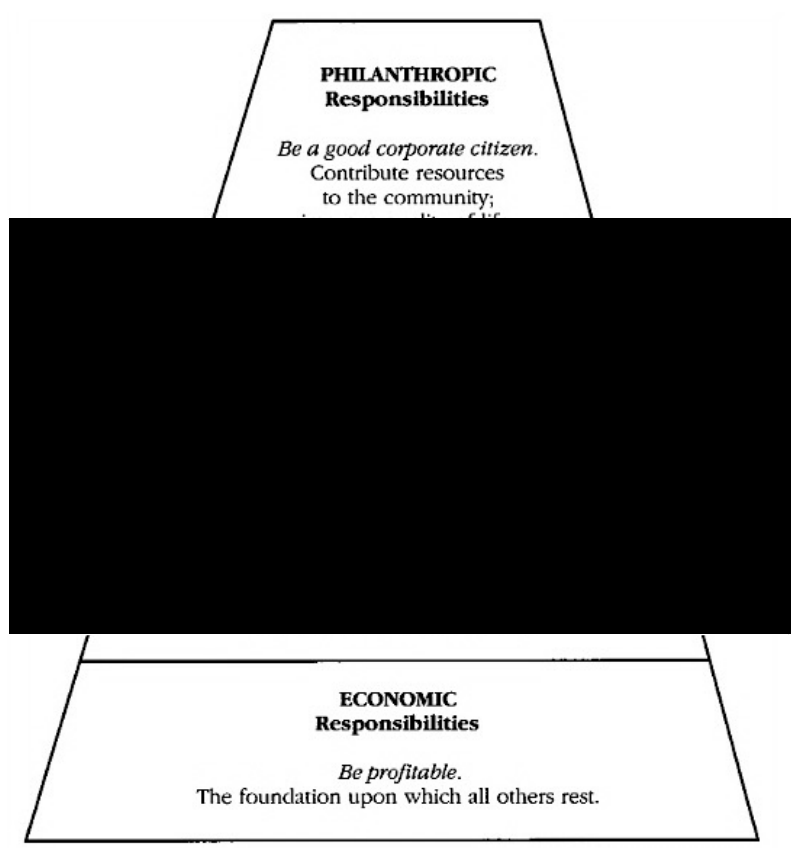

Carroll's four-part definition is pivotal for the CSR field, highlighting the key themes of CSR conceptualisation and motivations, both important considerations of this research. First, he refers to economic responsibility as a social consideration, important for sustainable development and an essential concern for managers. This aligns broadly with Friedman's (1970) perspective, who understood economic responsibility to go beyond merely profit-seeking behaviour. The pyramid structure of the domains also implies that there is some sort of sequential order, with economic viability being a prerequisite to other forms of responsibility. However, Carroll (2016) has recently clarified that despite the ethical responsibility being depicted as a single category, it should also be seen as a feature that is infused in each of the others domains, including the economic.

Following Carroll's developments, the preoccupation with defining CSR gave way to a focus on actors that are impacted by these responsibilities. The most notable contribution came from Freeman (1984) in his publication, Strategic Management: A Stakeholder Approach. It was at this point that 'stakeholder theory' was introduced, identifying that businesses have relationships with numerous groups who either impact, or are impacted by the actions of the business. They are therefore considered to have a stake in that business. The groups identified either had a direct internal impact such as owners, customers, employees and suppliers, or were 
external with an indirect impact, such as government and competitors (see Figure 3.4). Stakeholder theory provided valuable insights into business ethics by educating on the importance of relationships, to this day it remains a dominant theory and has a central role in this research.

Figure 3.4: Stakeholder Groups (Freeman, 1984)

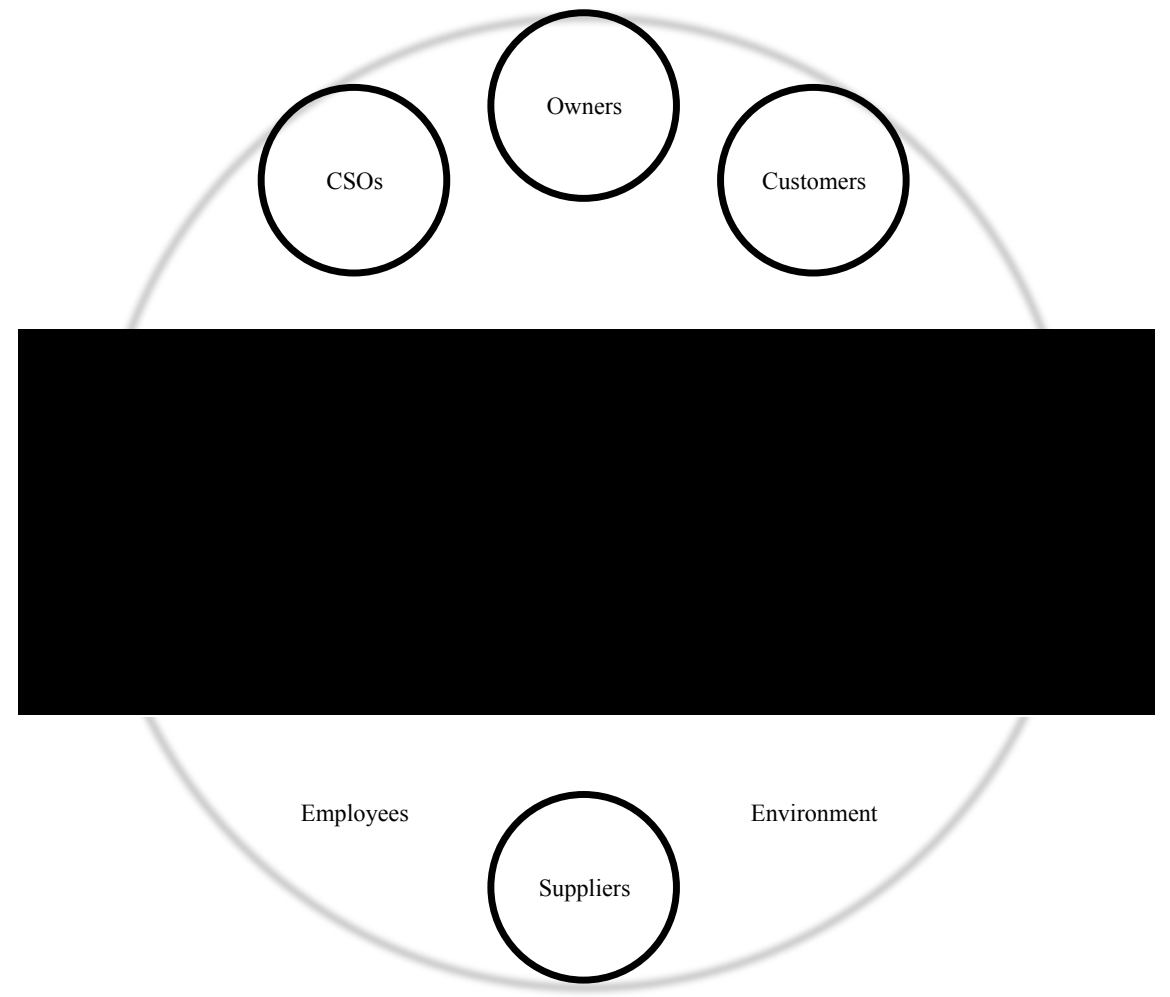

The 1980's saw progression of CSP, focusing on "achieving results or emphasizing the outcomes of socially responsible initiatives" (Carroll \& Shabana, 2010, p. 88). The concept was introduced previously by Sethi (1975) and developed by Carroll (1979), who looked to differentiate between CSR and CSP via three components of social responsibility, social responsiveness and social issues management. Wartick and Cochran (1985) also presented an evolved version of the CSP model, extending Carroll's three dimensions to include principles, processes and policies. On the other hand, Epstein's (1987) 'corporate social policy process' integrates business ethics, CSR and corporate social responsiveness as concepts that have been used to evaluate CSP. He proposes not an ad hoc system, but institutionalised system of individual and moral reflection in order to help improve the way businesses operate in the rapidly changing environment.

Finally, it was Aupperle et al. (1985) who first empirically explored the relationship 
between CSR and profitability. They utilised Carroll's (1979) definition to seek opinions of CEO's listed in the Forbes 1980 annual directory, in an attempt to measure CSR. The study confirmed that the domains of Carroll's definition held traction in the opinion of CEOs at that time, but results showed no evidence of a relationship between social responsibility and profitability. This opposed popular belief and prompted attempts to prove the business case, with differing results. As Lee (2008) explains, "the economic and social interests within organizations came closer and became much more responsive in the 1980's, but could not yet be tightly coupled together" (p. 60).

\subsection{5: The Business Case (1990's)}

The 1990's provided no radical contributions for the CSR literature, but saw the development of complimentary concepts such as corporate citizenship (CC), sustainability and continuation of the CSP debate. CC as a concept competed with CSR, yet Carroll argued that it was not distinct or unique and appeared to overlap with present themes. Sustainability and the environment also gained some interest, although as Carroll (2008) and Spence (2014) point out, there is ambiguity when it comes to the concept of sustainability and distinctions need to be made as environmental issues are often scientifically measured and legislated. Both of these concepts have their own extensive literature and therefore will not be summarised in this time frame.

However, as society became more concerned about the outcomes of CSR, focus moved from theoretical to more pragmatic concerns (Matten, Crane \& Chapple, 2003). CSP matured from the previous decades and attempted to reconcile the economic and moral schools of thought of CSR pursued, a concept that was coined the business case (Carroll \& Shabana, 2010). This resulted in a performanceorientated focus developing (Wood, 1991; Wood \& Jones, 1995) and the business case coming of age. Consequently, in the late 1990's CSR became a concept linked to complimentary strategy literature (Orlitzky, Schmidt \& Rynes 2003; Porter \& Kramer, 2002, 2006).

A distinct piece of work that emerged as a result of the previous decade was Wood (1991), who attempted to create a more useable model for CSP by integrating 
previously independent concepts. Wood critiqued Carroll's definition of CSR because of the supposed steps of responsibility, not viewing them as separate domains, but each a space where CSR can be engaged with. In order to address this concern she proposed multi-layered principles of CSR in her CSP model, whereby there are three levels of analysis. These levels include managerial discretion at an individual level, public responsibility at an organisational level and legitimacy at an institutional level (see Table 3.1).

Table 3.1: Principles of CSR (adapted from Wood, 1991)

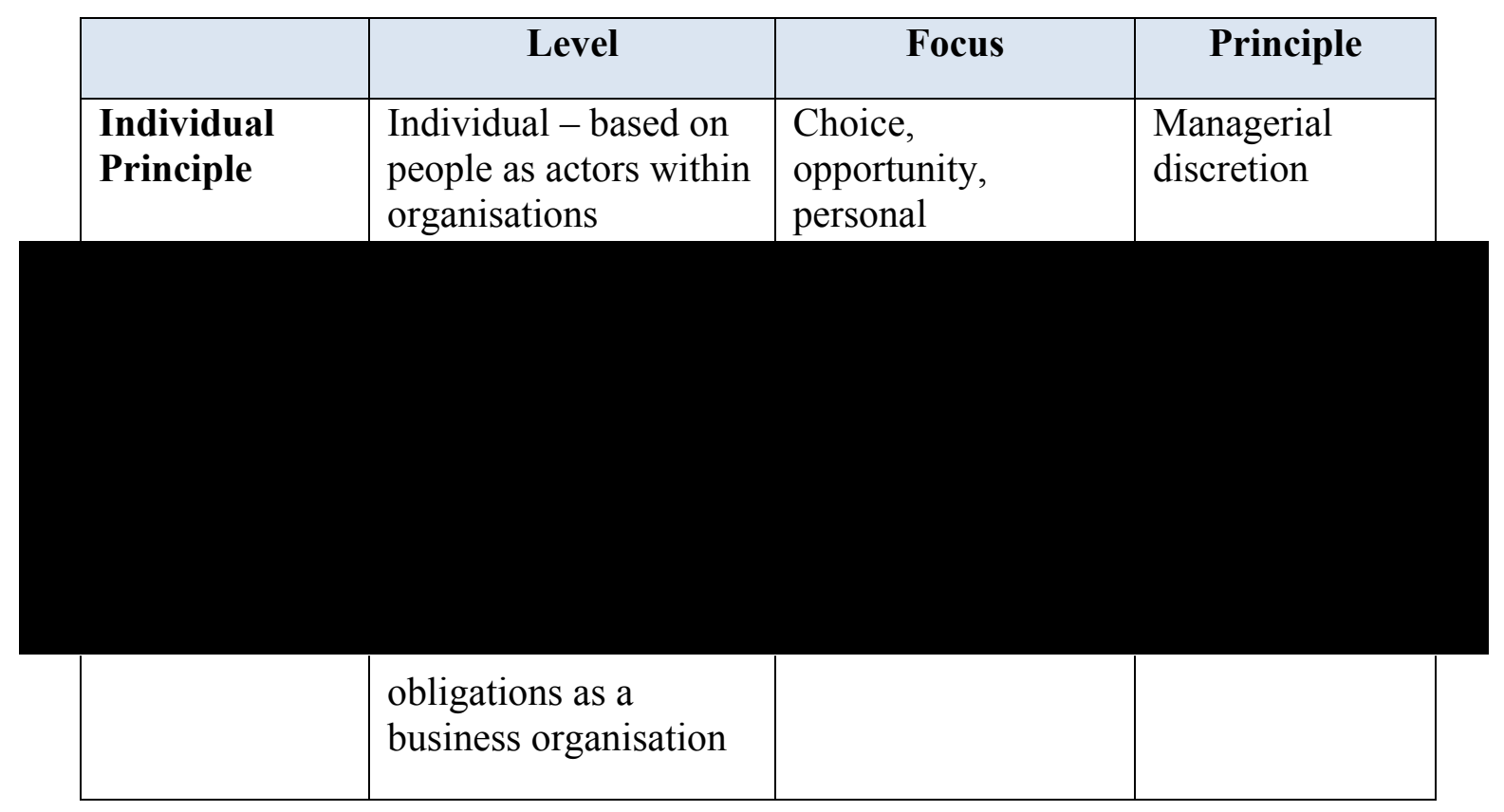

The institutional principle is based on the legitimacy and power that society grants to business, whereby if power is not used within reason, it will be lost in the long run. The organisational principle refers to businesses being responsible for actions when they might affect society in any way. Finally, the individual level is where managers are considered as moral actors and should act in accordance to this for every domain of CSR. Wood (1991) identified examples for each of these separate principles in relation to Carroll's four domains. From this perspective, the managerial level leaves scope for individual interpretation and choice when engaging with the CSR concept, concentrating on morals and reintroducing individual decision-making, These individual choices are undeniably influenced by the organisational and institutional context of which the business is embedded. The context and embeddedness of SMEs is a key consideration of this research and will be addressed 
in the following section.

Kang and Wood (1995), among others, (Kang, 1995, Sachs \& Ruehle, 2009) later reinforced the role of morals and managerial discretion when they reviewed and suggested updates for the hierarchy of Carroll's CSR pyramid. They criticised the "leading paradigm" (Schwartz \& Carroll, 2003, p. 504) and traditional view for placing primacy on economic responsibilities (Aupperle, 1984; Buccholz, 1991), a perspective derived from a distinctive trail from neoclassical economics whereby the firm is seen as a profit maximiser and rational actor, surviving due to their efficiency (Friedman, 1970). However, that view has long been contested (Freeman, 1984) and Kang (1995) and Baden (2016) argue specifically the dangers of the contention that ethical responsibilities are subservient to economic rationality.

It is suggested that by predicating economic responsibility before that of legal and ethical, the welfare of society is more likely to be marginalised for economic ends (Baden, 2016). Consequently, Kang and Wood (1995) flipped Carroll's pyramid and proposed a redrawn version, with moral responsibilities as the source of all CSR components and social and economic responsibilities following. From this perspective, only once the moral and ethical domains are fulfilled is the business free to pursue profit. This model recognises the paramount role of the individual and significance of context through a multi-dimensional understanding of CSR, a key consideration of this research. However, despite the value of this paradigm, the model failed to succeed in widespread application.

Nevertheless, Baden (2016) did acknowledge the value of their redrawn model, reintroducing the proposals by Kang and Wood (1995) nearly a decade later in her revised pyramid ranked as follows: ethical, legal, economic and philanthropic. She suggests that Carroll's paradigm has permeated "a business-centric notion of CSR" (p. 1) with economic precedence, considering that had Kang and Wood's redrawn model sustained widespread adoption, the CSR concept may have taken a different path. In consequence, there is a vital need for a shift to more socially responsible managerial mindsets and behaviours. Baden (2016) proposes "the greater role and power of business relative to government in the $21^{\text {st }}$ century necessitates an updating and reviewing of the priorities suggested by Carroll's pyramid of CSR" (p. 1) and "moral counter-balance" (p. 12) to the pervasive economic paradigm. 


\subsection{6: Strategic Orientation and Empirical Exploration (21st century)}

As a result of the above progressions, the $21^{\text {st }}$ century saw a new strategic orientation and wealth of empirical exploration. Whilst businesses were still dedicated to corporate giving and societal affairs, traditional philanthropy diversified to strategic forms of CSR and an emphasis on shared value. CSR became a concept that was pursued in areas that could enhance the long-term competitive advantage of businesses (McWilliams \& Siegal, 2001), with early advocates such as Lantos (2001). Porter and Kramer $(2002 ; 2006)$ also supported the strategic view and suggested the closer a company's corporate philanthropy to their competitive context, the more beneficial contributions and value creation for society.

In line with this perspective, Porter and Kramer (2011) introduced CSV, creating shared value, defined as "policies and operating practices that enhance the competitiveness of a company whilst simultaneously advancing the economic and social conditions in the communities in which they operate" (p. 66). The development of this concept was an attempt to bridge trust issues and transform social problems relevant to the business context into opportunities, a form of proactive CSR. This is in an attempt to find business legitimacy by solving problems and simultaneously driving profit, significant to proving the business case of CSR.

That being said, empirical research has produced varied results with regards to CSR and financial performance. For example, findings from the likes of Schreck (2011) and Orlitzky et al. (2003) support Porter and Kramer's (2002, 2006, 2011) strategic perspective and positive relationship between CSP and CFP across industries and contexts. However, McWilliams and Siegal (2001) only found a neutral relationship, although this still contends that CSR is legitimate and not detrimental to profit. Despite no cost difference of participating in CSR, the intangible benefits such as social capital (Russo \& Perrini, 2010; Sen \& Cowley, 2013) can have long run business benefits.

That being said, CSV was strongly critiqued by Crane, Palazzo, Spence and Matten (2014). They declared that the concept is unoriginal and follows a shallow conception of the businesses role in society. The most prominent issue was the view 
that the sole purpose of business is to create economic value for owners. It was claimed that CSV was just an example of stakeholder theory whereby accommodating important stakeholders assisted economic value. This was not the only time that stakeholder theory was referred to, Carroll and Shabana (2010) suggest that only when a business is able to pursue CSR with the support of stakeholders, can there be a business case for CSR.

Stakeholder theory remained popular throughout previous decades and into the $21^{\text {st }}$ century. The enduring popularity resulted in widespread adoption and multiple extensions and adaptations of the theory. It was not only featured in the business case and legitimacy debates, but comparative research with social capital (Perrini, 2006; Russo \& Perrini, 2009) and adopted to establish a stakeholder salience model (Sen \& Cowley, 2013) amongst others. As Lange and Washburn (2012) sum up nicely, "the external expectations for social responsibility is rooted in the perceptions of the beholder" (p. 300), placing emphasis on stakeholders and recipients of CSR and the value of this theory for CSR developments.

A further theme that was carried into this era was business ethics. With a reemphasis on morals from Kang and Wood (1995) and increasing ethical challenges for business, it was thought that business ethics would remain a meaningful issue for CSR. Research included the likes of Carroll and Shabana (2010) who drew from Wood's (1991) individual level of focus and proposed three types of moral management: immoral, moral and amoral. Windsor (2006) also proposed three key approaches to CSR: ethical, economic and corporate citizenship and Garriga and Melé (2004) mapped four CSR perspectives inclusive of ethical. With a rise in the strategic orientation of CSR, business ethics remains of integral importance to root the CSR debate as an inherently ethical topic. The motivations and perspectives of CSR are of integral importance to this research and will be reflected on in the following section.

\subsection{7: Summary}

In summary, the chronological overview of CSR literature has revealed a number of key themes and gaps in the literature. There are five significant concepts that have considerably influenced the following research. The first of these is the role of 
context for CSR. It was proposed by Wood (1991) that CSR should be analysed via a multi-dimensional framework. She acknowledges that full comprehension of the concept is not possible on a single level of analysis due to changing contexts of which businesses are embedded. As the developments of the concept changed to reflect the changing reality of society, so does CSR in relation to the business context. Previous research does not fully accommodate for the influence of context, whereby factors from individual, organisational and institutional levels are accounted for. Consequently, this will be a consideration of the following research.

The second concept was the role of the manager. The individual level of analysis proposed by Wood (1991) determines that managers are moral actors and have scope for individual interpretation and choice during decision-making. Traditional thinking in the foundational phase of CSR was philanthropic and broadly founded on the morals of business leaders, yet the institutionalisation of such social responsibilities to government and the profit maximisation motive that followed, muted the importance of managerial discretion. Whilst deregulation in the 1980's placed a greater emphasis on personal responsibility in business, there is still a lack of focus on individuals as ethical decision-makers and key influencers of CSR. In this research, attempts will be made to refocus on owner-managers to address this gap.

In relation to managerial influence, a further gap in the literature is a disregard for the personal and relational aspects of business. As identified above, the concept of CSR often reflects the reality of the manager and business. Consequently, stakeholder relationships and personal connections are likely to play a dominant role in shaping interpretations and application of the CSR concept. Whilst stakeholder theory has withstood enduring popularity following the questionable practices of MNCs in the 1980's, attention on relationships is limited. Social capital theory has been introduced to the landscape of CSR, yet there is still a need to appreciate the role that relationships play in molding our understanding and interpretations of CSR.

However, one of the most distinctive reflections from the literature is the preoccupation with large business CSR research. The majority of theoretical developments and extant research is derived from this context, somewhat disregarding the contribution of small and medium-sized enterprises (Jenkins, 2004; 
Lähdesmäki \& Suutari, 2012; Spence, 2016). This is surprising considering the collective importance of the SME sector. Research suggests that characteristically, SMEs are significantly different to large businesses, with suggestions that scaled down CSR theories do not translate or adequately represent the SME conception. The following research responds to this gap in the literature by focusing specifically on the way that SMEs make sense of CSR.

The final observation of literature is the enduring presence of the economic perspective. Whilst there has been a shift from Friedman's (1970) profit maximisation paradigm, there is still a preoccupation with establishing a link between CSR and corporate outcomes. For example, the most widely accepted CSR definition from Carroll (1979) predicates other responsibilities on that of the economic, showing disregard of the moral foundations of the concept. Instead, the debate has turned to rational economic justifications, strategic activity and competitive advantage. However, this constricted research pathway of CSR is being challenged and there have been recent calls to reclaim the ethical foundations of CSR (Baden \& Harwood, 2013; Brooks, 2010) to recognise the new social role of business (Roche \& Jakub, 2017). The following research develops the literature in its own right by responding to this call and concentrating specifically on two moral perspectives of CSR.

To conclude, now that the conceptual foundation of CSR has been established and key gaps in the literature identified, the following section will address the main research context of CSR in SMEs.

\section{3: Corporate Social Responsibility (CSR) in Small and Medium- sized Enterprises (SMEs)}

As the previous section identified, social responsibility in SMEs still remains a comparatively under-researched aspect of CSR (Jenkins, 2004; Lähdesmäki \& Suutari, 2012; Spence, 2016; Spence \& Rutherfoord, 2003), despite that in Europe, SMEs account for over 99\% of enterprises (Baden, Harwood, \& Woodward, 2011; Baden \& Harwood, 2013). Having said that, attempts have been made by numerous 
academics to summarise the CSR literature for SMEs (Hannafey, 2003; Kechiche \& Soparnot, 2012; Lepoutre \& Heene, 2006; Spence, 1999; Spence, 2007; Spence \& Painter-Morland, 2010; Thompson \& Smith, 1991; Vázquez-Carrasco \& LópezPérez, 2013), with an increased interest in this field resulting in competing and complimentary literature emerging from a range of disciplines. The following section will continue the discussion by providing an overview of academic contributions relevant to the research and SME context.

\subsection{1: SMEs and their Significance}

According to the Department for Business Innovation and Skills 2014 statistical release, there were just over 5.2 million small businesses in the UK, with $99.9 \%$ classified as SMEs (Department for Business, Innovation and Skills, 2014). These figures alone emphasise the importance of SMEs in the UK, with similar findings recurring worldwide. The Federation of Small Business (FSB) declared the acronym SME as an "intrinsic part" of our current vocabulary $(2011$, p. 8), yet small businesses are "not a common starting point for business and society researchers" (Spence, 2014, p. 2). This is surprising considering that they are a "major source of entrepreneurial skills, innovation, and contribute to economic and social cohesion" (Commission of the European Communities, 2005, p. 3).

Historically, studies into small business social responsibility have been characterised by the application of scaled down large firm CSR theories (Jenkins, 2004; Russo \& Perrini, 2010). This has resulted in unfounded assumptions about the way SMEs behave. There have however been important contributions in recent years that have seen an increase in SME theories and more consistent definitions (Jamali, Zanhour \& Keshishian, 2009; Jenkins, 2004; Moore \& Spence, 2006; Spence, 1999; Spence, 2016), specifically the European definition in this case.

\subsection{2: SME Definition}

Currently, there is not one globally accepted SME definition, the term is broadly used and incorporates a diverse range of businesses. In the past, this heterogeneity has deterred the adoption of one definition, leaving it open to interpretation and 
causing problems for SME research on a global scale (Spence, 1999; Curran \& Blackburn, 2001; Jenkins, 2006). For example, Spence (1999) showed concerns for the early US small business ethics research, whereby the definition has been based on as few as 25 employees (Wilson, 1980), less than 1000 people employed (Dunfee, Bowie, Hennessey, Nelson \& Robertson, 1991; Robertson, 1993) and up to 1500 employees (Holliday, 1995). Due to the prominence of the US in early CSR research the validity of these findings need to be acknowledged in comparison to recent research.

The Bolton Committee Report was one of the first prevalent sources of understanding to overcome these issues. Bolton (1971) proposed an economic and statistical explanation that gained salience and guided the formulation of a European definition. The current European guidelines (European Commission, 2015) determine an SME as having equal or less than 250 employees, with a turnover below $€ 50$ million or a balance sheet below $€ 43$ million (see Table 3.2). Due to the contextual relevance, in order to align with the FSB understanding of an SME and by recommendation from other researchers (Curran \& Blackburn, 2001; Spence \& Perrini, 2009), it is this definition that was adopted for the purposes of the research.

Table 3.2: European Commission SME definition

\begin{tabular}{|l|l|l|l|l|}
\hline Company category & Employees & Turnover & or & Balance sheet total \\
\hline Medium-sized & $<250$ & $\leq € 50 \mathrm{~m}$ & $\leq € 43 \mathrm{~m}$ \\
\hline Small & $<50$ & $\leq € 10 \mathrm{~m}$ & $\leq € 10 \mathrm{~m}$ \\
\hline Micro & $<10$ & $\leq € 2 \mathrm{~m}$ & $\leq € 2 \mathrm{~m}$ \\
\hline
\end{tabular}

(European Commission, 2015)

\subsection{3: CSR Terminology}

It is widely claimed that CSR is not a suitable term to describe social responsibility in SMEs (Murillo \& Lozano, 2006). In a critical analysis of the term Jenkins (2004) establishes that small businesses do not identify with the word 'corporate', disengaging from the term as a whole. In theory an SME is a corporation, yet in practice the word has consistently been used to refer to large firm research, taking 
away meaning and relevance for SMEs. As is continually emphasised, "small firms are not little big firms" (Tilley, 2000, p. 33), warranting language more specific to the small business context.

However, following a review of the literature there is still a lack of consensus on an alternative term that adequately portrays the concept of CSR in SMEs (Jenkins, 2004; Murillo \& Lozano, 2006). The critique of CSR language has resulted in a proliferation of related terms (see Table 3.3), although few of these have gained traction. Instead, they have diversified the concept and added to the ambiguity.

\section{Table 3.3: Terminology Table for CSR in SMEs}

\begin{tabular}{|l|l|}
\hline Terminology & Author \\
\hline $\begin{array}{l}\text { Business Community } \\
\text { Interaction }\end{array}$ & Jenkins (2004) \\
\hline $\begin{array}{l}\text { Small Business Social } \\
\text { Responsibility }\end{array}$ & $\begin{array}{l}\text { Chrisman \& Archer (1984), Lepoutre \& Heene (2006), } \\
\text { Spence (2014), Spence (2016) }\end{array}$ \\
\hline $\begin{array}{l}\text { Responsible } \\
\text { Competitiveness }\end{array}$ & Murillo \& Lozano (2006) \\
\hline $\begin{array}{l}\text { Responsible Business } \\
\text { Behaviour }\end{array}$ & Avram \& Kühne (2008) \\
\hline $\begin{array}{l}\text { Responsible Business } \\
\text { Practise }\end{array}$ & Moore, Slack \& Gibbon (2009) \\
\hline Responsible Business & Moore \& Spence (2006), Southwell (2004) \\
\hline Social responsibility & Spence \& Perrini (2011) \\
\hline
\end{tabular}

Such disengagement and definitional ambiguity is likely to deter the adoption of a term that more suitably reflects CSR in SMEs. This risks a lack of acknowledgement of the true value of SME's social engagement. It seems apparent from the literature that there is a need to stop the introduction of new terms and instead progressively adapt the CSR term to one closer to the SME reality (Murillo \& Lozano, 2006). Spence (2016) has more recently adopted 'small business social responsibility', originally used by Chrisman and Archer (1984). This adaptation maintains consistency with original CSR language, whilst distinguishing the SME 
conception. Consequently, it may be a more appropriate alternative to progress SME specific research. However, the purpose of this research is not to propose CSR terms, but to consider terminology in relation to the SME conceptualisation of CSR.

Baden and Harwood (2013) do this by reflecting on the underlying assumptions of language. This is depicted in the following passage when they say, "our ideas are dependent upon the words we use to describe them" and "the terminology we use influences how our world is perceived" (p. 621). They share similar thoughts with Wicks et al. (1994, p. 475) who consider it "essential that we remain continually aware of the dominant metaphors we use to describe ourselves and our practises because they often shape us in ways that we don't initially recognize or desire".

However, current terminology is claimed as "not fit for purpose" (Baden \& Harwood, 2013, p. 615) for SMEs because the economic focus of the business case co-opts the ethical concept of CSR. The disengagement identified in the research is explained as a result of "connotations that may be antithetical to their primary purpose of encouraging more ethical and pro-social policies and behaviour" (p. 615). The above reflections emphasise the importance of language for CSR research and the need for acknowledgement in future research.

\subsection{4: SME Research Agenda}

\subsubsection{1: Size and Characteristics}

Early SME approaches for CSR emerged in the US following popular contributions from the likes of Milton Friedman (1970), Archie Carroll (1979) and Edward Freeman (1984). This initial research focused on the scaling down of large firm CSR theories. Whilst this was useful to open up the small business ethics debate, limitations were identified (Spence, 1999) and the inappropriateness of scaled down theories claimed (Spence \& Rutherfoord, 2003). Jenkins (2004) argues that CSR initiatives aimed at SMEs were therefore based on incomplete assumptions considering that the characteristics of SMEs are distinct from large businesses.

It was following highly publicised incidents of unethical behaviour in the 1990's (Vogel, 1992) and a European Ethics Network theme on Market Morality and 
Company Size (Harvey, van Luijk \& Corbetta, 1991) that the business ethics debate for SMEs gained salience in Europe. The increasing interest in CSR related topics resulted in the development of surrounding literature and a number of important contributions. Spence (1999) considered the exploratory approach adopted in Europe as more appropriate than the overuse of quantitative surveys that objectified CSR in the US (Brown \& King, 1982; Chrisman \& Fry, 1982; Murphy, Smith \& Daley, 1992). That being said, European literature to date is still described as "a distant relative of the main field" (Spence, 2016, p. 45), with the theoretical development of the concept still embryonic.

Appendix 3.1 provides a comprehensive overview of the key research themes, disciplinary perspectives and publications that have contributed to the conceptual and theoretical development of CSR in SMEs so far. This body of literature was initiated with a focus on size attributes (Dandridge, 1979; Fassin, 2008; Fuller \& Tian, 2006; Jenkins, 2006; Lepoutre \& Heene, 2006; Spence, 1999; Spence \& Painter-Morland, 2010), which helped outline general characteristics of SMEs unique to their larger counterparts (see Table 3.4). This resulted in a challenging of the exclusive concentration on large firms in business ethics (Spence, 1999), with attention turning to the critique of conventional CSR theories.

Table 3.4: Characteristic Differences between Large Corporate Firms and SMEs

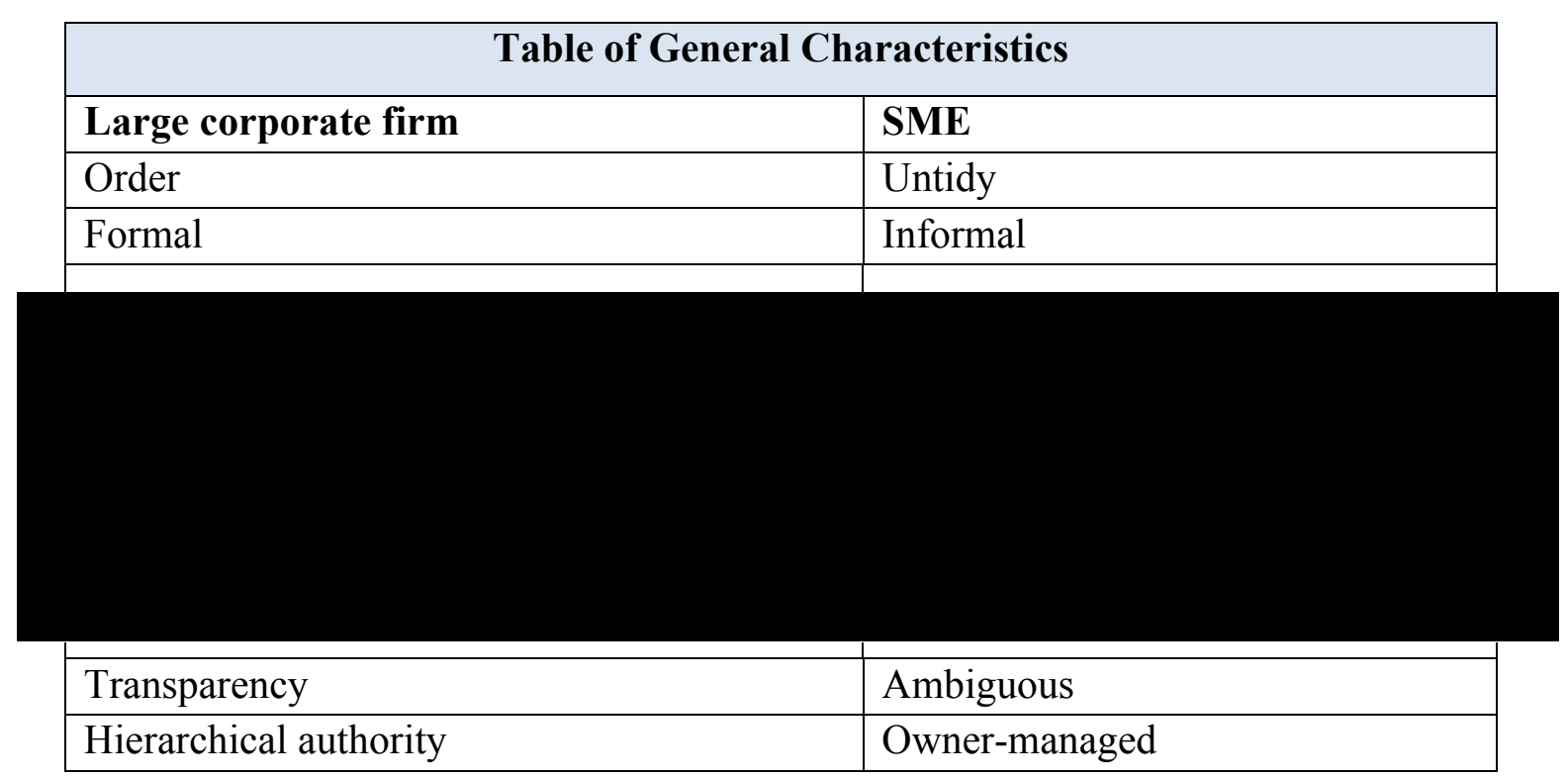




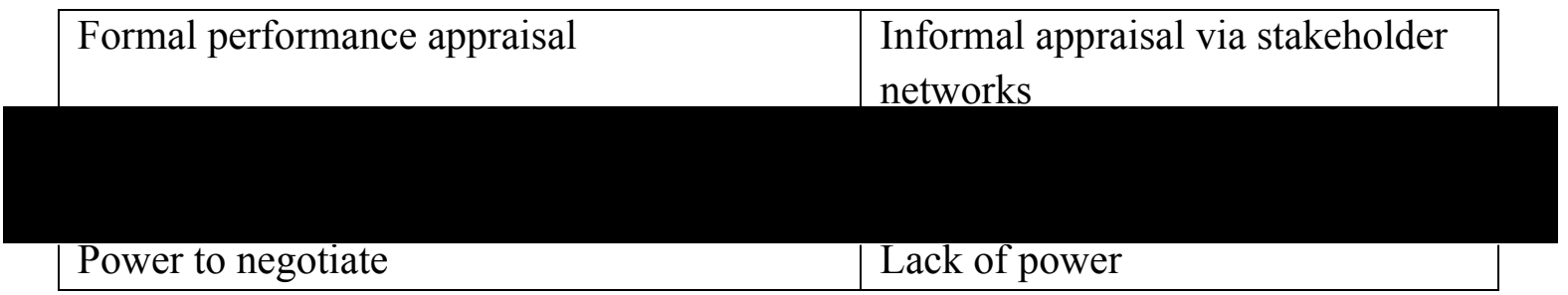

Compiled by author, adapted from Spence (1999), Lepoutre and Heene (2006) and Jamali et al. (2009).

The distinguishable features of SMEs are known to impact the motivations, engagement and nature of socially responsible activities in SMEs (Spence \& PainterMorland, 2010). Jenkins (2004) was one of the first to identify the disparity of CSR approaches in Table 3.5, demonstrating how important it is to acknowledge size characteristics and adopt appropriate methodologies. However, Spence (1999) claims that little of what was known about SMEs and their novel attributes were actually adopted in early research. Therefore, one of the key objectives of this study is to address this concern by adopting an appropriate qualitative methodology to explore how SME owner-managers socially construct and make sense of their role in society.

Table 3.5: Table of Comparative CSR Characteristics (adapted from Jenkins, 2004)

\begin{tabular}{|l|l|}
\hline \multicolumn{2}{|c|}{ Table of CSR Characteristics } \\
\hline Large corporate firm & SME \\
\hline Who & Who \\
\hline
\end{tabular}

\begin{tabular}{|l|l|}
\hline The business case & \\
\hline How & Proven business case lacking \\
\hline
\end{tabular}




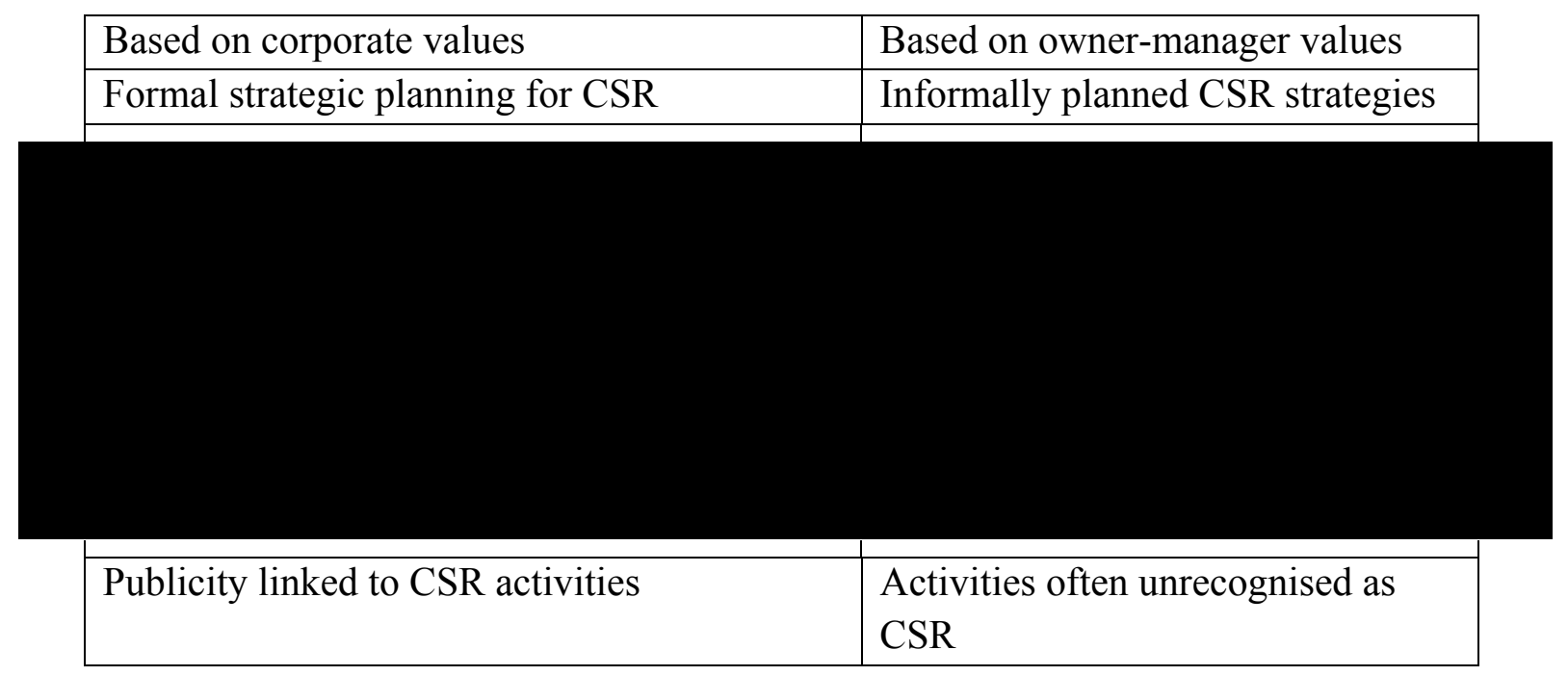

\subsubsection{2: CSR Perspectives and Motivations}

Following the focus on size characteristics, there was an attempt to establish an SME perspective of CSR. In 1980, Erika Wilson asked the question, "What is the small businessperson's stand on business responsibility in society?" (p. 17). She concluded that there is a dominance of the profit motive (Chrisman \& Archer, 1984), whereby "social issues are only important to the extent that they have an indirect impact on profit" (Spence, 1999, p. 167). However, subsequent research diverged from this perspective (Brown \& King, 1982; Chrisman \& Fry, 1982; Chrisman \& Archer, 1984), suggesting that social engagement is driven by much more than profit and "small businesses perform quite well in discharging social duties" (Chrisman \& Archer, 1984, p. 47).

Since this early research a number of frameworks have been developed to help categorise the key CSR perspectives for SMEs (Brønn \& Vidaver-Cohen, 2009; Campin et al., 2013; Campopiano, De Massis \& Cassia, 2012; Murillo \& Lozano, 2006; Spence \& Rutherfoord, 2001; Windsor, 2006; Wickert, Scherer \& Spence, 2016). These frameworks most commonly operate on a continuum from economic to altruistic drivers. The economic perspective proposed by Wickert et al. (2016) contends that CSR is driven by instrumental motives, whereby engagement is in pursuit of financial benefits or competitive advantage (Campopiano et al., 2012; McWilliams, Siegal \& Wright, 2006; Tang, Hull \& Rothenberg, 2012). 
A second perspective proposed by Wickert et al. (2016) is the relational or institutional. Rather than use the term "institutional", due to the confusion between levels of analysis as highlighted by Wood (1991), this will be referred to as 'organisational'. External demands such as stakeholder expectations in the shortterm (Mitchell, Agle \& Wood, 1997; Mitchell, Agle, Chrisman \& Spence, 2011) and engagement with legitimate (Campbell, 2007), socially acceptable activities in the long term are key drivers included in this perspective of CSR (Aguilera, Rupp, Williams, \& Ganapathi, 2007). Considerations could also be made for the proactive use of CSR for relational development in SMEs, otherwise known as social capital (Murillo \& Lozano, 2006; Russo \& Perrini, 2010).

The third and final perspective that Wickert et al. (2016) derived from the literature is the ethical perspective. This is similar to Aguilera et al. (2007) and their morally motivated domain, in that it contends a CSR perspective that is driven by moral considerations (Baden \& Harwood, 2013; Donaldson \& Dunfee, 1994; Garay \& Font, 2012). The need to reclaim the importance of moral foundations for CSR in SMEs is argued by Baden and Harwood (2013) amongst others (Brooks, 2010; Nijof $\&$ Jeurissen, 2010; Spence, 2016), who contest the profit motive and suggest that the term CSR has "strayed from a focus on societal good" (p. 617). Consequently, there is a need to regain understanding of the deeper moral roots of CSR because "once CSR loses its foundation in ethics it becomes not only irrelevant, but counterproductive (p. 617).

A significant proportion of the small business CSR literature is still preoccupied with establishing a link between CSR and organisational success. However, it is argued that a refocus on the social sciences is more important than advancing the business case (Spence, 2016). This is because the emphasis on instrumental outcomes "drives out the intrinsic motivation for engaging in CSR" (Nijof \& Jeurissen, 2010, p. 618) and second because profit maximisation is generally not a main priority or driver of social responsibility for SMEs. There is increasing evidence to suggest that SMEs are driven by much more than economic rationality and profit, referring to the important role of the owner-manager (Spence, 2016). Therefore, a key aim of this research is to refer back to the moral philosophy grounding CSR, specifically to 
establish the intrinsic motivations of owner-managers that drive the SME perspective of CSR.

\subsubsection{3: Stakeholder Relationships}

A distinct feature of SMEs is the nature of stakeholder relationships. Studies have attempted to establish how these differ from large firms and impact CSR perspectives. The first notable difference is the personal nature of business (Fuller \& Tian, 2006). Due to the integrated, human aspect of SMEs (Jenkins, 2004), business relationships and connections are often inextricable and unavoidably transcend into personal boundaries (Chrisman, Chua, Pearson, \& Barnett, 2012; Spence, 2007; Spence, 2014). SMEs seek legitimacy and informal appraisal via these immediate stakeholder groups, meaning that their influence significantly shapes CSR engagement (Murillo \& Lozano, 2009). Personal relationships can also act as a competitive advantage for SMEs over large firms (Lepoutre \& Heene, 2006; Spence, 2007), emphasising the importance of informal connections for business survival.

SMEs are also defined by their embeddedness within local communities. Their focus is less on society and more on the communities within which they operate. As Spence (2014) explained, Lähdesmäki and Suutari (2012) have shown that "proximity to the local community embeds the firms in a system of reciprocity with its neighbours" (p. 382). This integrated nature of relationships means that often SMEs conceptualise CSR via their contributions and engagement within the community. Jenkins (2004) explains the enlightened self-interest logic of CSR whereby engagement is recognised and rewarded by stakeholders in the location they are connected to, legitimising the SME business activities. Alternative research has suggested that SMEs are "fortress enterprises" (Curran \& Blackburrn, 1994, p. 113) and highly disconnected from the community in which they operate. However, this view has been "robustly countered" (Spence, 2016, p. 9).

The SME literature is infused with research that draws on stakeholder theory (Jenkins, 2004; Jenkins, 2006; Spence, 2016; Tang \& Tang, 2012) and social capital theory (Perrini, 2006; Sen \& Cowley, 2013; Spence \& Schmidpeter, 2003; Spence, Schmidpeter, \& Habisch, 2003; Russo \& Perrini, 2010). These theoretical 
perspectives have presented a valuable lens for CSR by recognising the importance of stakeholder relationships. They acknowledge that SMEs "have a responsibility to all those who affect or are affected by their actions" (Spence, 2016, p. 5) and are reliant on "complex webs of relations" (Russo \& Perrini, 2010, p. 209) and elements of social capital such as trust, integrity, reciprocity and networks. Such relational interconnectedness (Jenkins, 2006) is an important contributor facilitating business success (Lähdesmäki et al., 2017) and shaping CSR.

It was Perrini (2006) who initiated comparative studies of these theories, suggesting that social capital may be more appropriate for SMEs in comparison to stakeholder theory. Sen and Cowley (2013) found this to be true, suggesting an alignment to the fundamentals of social capital. However, Russo and Perrini (2010) establish that whilst SMEs might be predisposed to one perspective, there is a common consensus that both are drawn from simultaneously. This means that stakeholder theory cannot be disregarded for SMEs and should inform management in line with social capital considerations. Jenkins (2006) had also previously advocated stakeholder theory as an appropriate framework, but acknowledged a need for development to accommodate all forms of SME. Spence (2016) addressed this need with theoretical developments that form the basis of this research. These will be covered in more detail in section 3.3 .5 below.

\subsubsection{4: Informal Nature of SMEs}

A further distinguishing aspect is the informal characteristic of SME business operations (Jenkins, 2004; Murillo \& Lozano, 2006; Spence, 1999; Spence, 2016; Wickert et al., 2016). The lack of bureaucratic controls allows freedom to be intuitive and responsive to stakeholder expectations and changing societal landscapes. This aligns with the reactive CSR typology proposed by Torugsa, O'Donohue and Hecker (2013) in their continuum that ranges between reactive and proactive CSR behaviour. It also shows lineage to the corporate social responsiveness literature (Ackerman, 1973; Ackerman \& Bauer, 1976; Wood, 1991), whereby the debate shifted to a more managerial approach, in line with the integral role of the owner-manager in SMEs. 
In keeping with this theme, informality is also a trait of CSR in SMEs (Jenkins, 2004). Research suggests that engagement is not budgeted for, is small-scale, informally planned and implemented on an ad hoc basis (Jenkins, 2006). SMEs do not structure or strategise such engagement for a number of reasons. This is primarily due to a lack of resources such as specialist staff and time (Fassin, 2008), but also because they do not recognise these activities as CSR. However, SMEs are congratulated on their flexibility when it comes to social engagement (Spence, 2016), as they can adhere to the needs of their stakeholders in a more integrated and timely manner compared to larger businesses. As Fassin (2008) explains, CSR is not something "intended to be restricted to a specialist team" (p. 370).

However, recent literature indicates the increasing pressure for SMEs to formalise and validate their social and environmental engagement via outbound communication mechanisms and methods of social reporting. Some examples of reporting are mission statements, written policies, codes of conduct or forms of systematic measurements (Fassin, 2008). However, Russo and Perrini (2010) talk of the "ineffectiveness of formal tools such as codes of conduct and social and ethical standards" as they "often require a large proportionate investment of time, finances, and energy from small firms" (p. 210).

Recent work by Wickert et al. (2016) found that CSR 'walk' and 'talk' were incongruent, whereby large businesses extensively talk CSR, yet implementation lags behind (Baumann-Pauly, Wickert, Spence, \& Scherer, 2013). On the other hand, for smaller businesses, CSR is often silently implemented with limited communication and low visibility (Nielsen \& Thomsen, 2009). In line with these findings, Fassin (2008) emphasises that "reports do not constitute the validation for real CSR, nor the proof of superior ethical behaviour" (p. 364). It is therefore wrong to assume that SMEs are not ethical or engaged with CSR due to an absence of reporting.

Jenkins (2006) did propose the use of small business CSR champions to promote CSR and increase engagement within SMEs. However, the above research shows that SMEs are already implementing CSR; they are just not formalising or communicating it. Murillo and Lozano (2006) therefore acknowledge that the true 
challenge for future research is "to find tools differentiated from the more formalised and established ones used by large enterprises" (p. 228). This is in order to ensure relevance for SMEs and to disseminate the valuable contribution of SMEs.

\subsection{5: Theoretical Frameworks and Perspectives}

The following section will discuss theoretical perspectives appropriate to advance the CSR research agenda for SMEs. There is currently an absence of SME specific research that brings the CSR debate back to the social sciences, with concerns that it has lost its moral foundations. As has been mentioned in Chapter Two, a key contribution of this research is referring back to the moral philosophy grounding CSR. Theoretical frameworks have been adopted that compliment the unique characteristics and non-homogenous nature of SMEs, drawing specifically from two moral perspectives identified as appropriate by Spence (2016). The following section outlines how the above concerns are addressed and how CSR theory can be advanced, referring specifically to the redrawn CSR models adopted to shape this research.

\subsubsection{1: Stakeholder Theory}

As identified in Chapter Three, Spence $(2014,2016)$ emphasises the appropriateness of the ethic of care and moral proximity for size sensitive CSR research. She calls for future literature to incorporate these moral perspectives and adopts them herself to redraw two core CSR theories. The first of these is stakeholder theory, most commonly associated with Freeman (1984) and redrawn by Wicks et al. (1994) from a feminist perspective. The review of SME literature above identifies the enduring value of stakeholder theory for CSR in SMEs (Jenkins, 2006; Russo \& Perrini, 2010; Tang \& Tang, 2012) with Sen and Cowley (2013) describing it as "pivotal for investigating a management issue such as CSR" (p. 29). Spence herself advocates its adoption due to the conceptual breadth and widespread acceptance in literature and practice.

Her redrawing of stakeholder theory underlies the view that "people are inextricably embedded in context" (Spence, 2016, p. 6). Accordingly, she identifies four 
elements aligned with the ethic of care that can "enhance the use of stakeholder theory" (p. 13) in SME research (see Table 3.6). The first of these refers to the central position of the owner-manager as legitimate decision maker. The second is that SMEs are not autonomous and more likely to be dictated by other organisations rather than as a central force in their own right. The third suggests that SMEs have "a different generalized set of stakeholders" (p. 29), proposing that organisations are arenas of both the public and private spheres. Accordingly, Spence outlines a set of key stakeholders typical to an SME. The final amendment refers to the influence of proximity on stakeholder salience as an added aspect to the urgency dimension.

\section{Table 3.6: Four Enhancements of Stakeholder Theory for SMEs (adapted from} Spence, 2016)

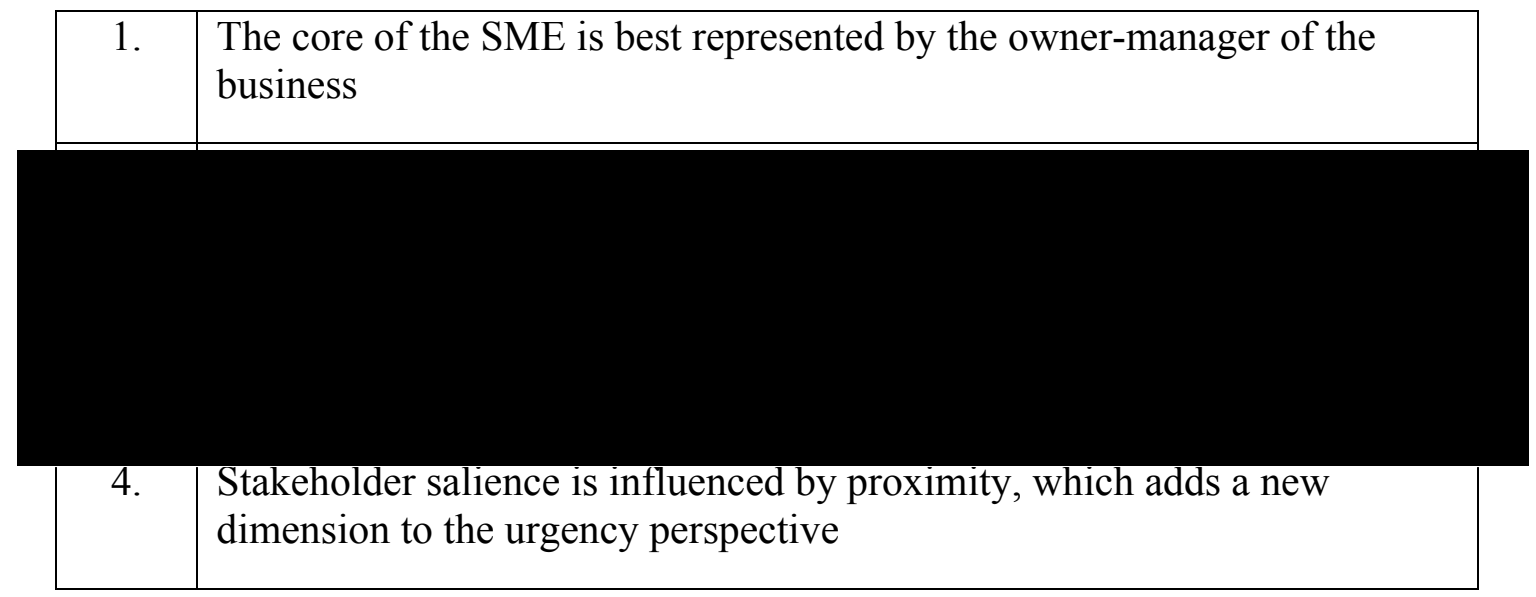

\subsubsection{2: Small Business Social Responsibility (SBSR) Pyramids}

The second theory redrawn by Spence (2016) was Carroll's (1991) CSR pyramid. Despite some reconstructions of the model over the years (Baden, 2016; Kang \& Wood, 1995; Schwartz \& Carroll, 2003), the redrawing for SMEs is novel. There were two main areas of adaptation for the pyramid; the four domains and the focus on a specific context, not generalised society (see Figure 3.5). Unlike Carroll, Spence considers the ordering of the pyramid as important, with the base representing the fundamental starting point of CSR and the apex indicating responsibilities that are desired but not essential. The first area of reflection appreciates the contextualised nature of SMEs, referring to four key stakeholders that SMEs are most likely to be accountable to: the self and family, employees, the 
local community and business partners. This is in contrast to the individual pyramid representing general societal responsibilities. However, she acknowledges that these stakeholders may change dependent on the SME and their context.

The four domains of the pyramid were also amended from economic, legal, ethical and philanthropic. The first development redraws the masculinist domains of economic and legal to survival. For SMEs, financial stability is more fragile and dependent on externalities such as key stakeholders. Ethical was also considered as an archetypal masculinity interpretation, referring to ethics with regards to rights, justice and fairness. This was replaced with an ethic of care in order to make explicit the care perspective in stakeholder relationships, particularly towards those in close proximity. Philanthropy is the only domain remaining the same. Its contribution is clarified due to the inclusion of responsibility to those beyond personal relationships, not previously accommodated by the ethic of care domain. Finally, personal integrity is included as a new domain, justified because of the integral role of the owner-manager and their influence on CSR decisions.

Figure 3.5: Small business social responsibility (SBSR) pyramids (Spence, 2016, p. 17)

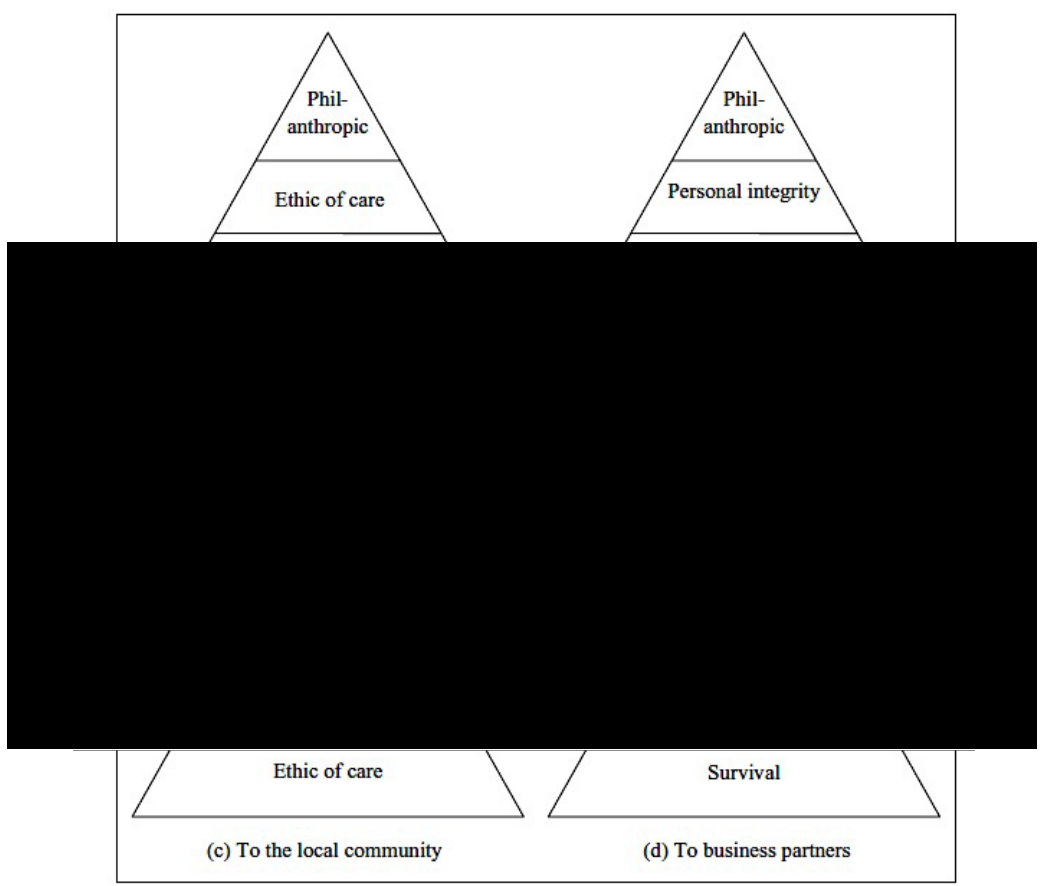


A key aspect of this research is evaluating the role of the ethic of care and moral proximity for CSR research in SMEs. Whilst these theories have been previously adopted, application to the SME setting is comparatively new. Spence's models are one of the first attempts to adapt core CSR theory in a way more appropriate for smaller businesses. Therefore, the aim of this research is not of grounded theory or to propose drastically new models, but to use the redrawn stakeholder theory and SBSR pyramids to gauge the empirical appropriateness of the moral perspectives. The emergent methodology allows for themes to surface with the possibility for confirmation and enhancement of theory. This potential for re-interpretive work and renewed exploration for CSR in SMEs is a significant contribution in itself.

\subsection{6: Analytical Perspective for CSR in SMEs}

Most business decisions are influenced by a number of micro, meso and macro factors (Corbin \& Strauss, 2008). Despite variances in descriptions (Kang \& Wood, 1995; Spence, 1999; Wood, 1991), micro influences can commonly be described as those relating to the individual. Meso influences are described as those linked with the organisation and their relationship with society and macro factors are those associated with the societal context in relation to economic, cultural and political influences. These levels are not mutually exclusive, but interrelated and influence each other. Understanding is therefore enhanced when there is acknowledgement of the interplay between these levels that shape CSR.

A multi-level analysis is consequently favourable for social constructionist research. It provides a framework to investigate different aspects of the context in which the social phenomenon exists. This responds to the need for a "context respectful" (Spence, 2014, p. 377) research approach for CSR in SMEs. Not only does it allow for the institutional dynamics of society that influence the organisational context, but acknowledges the important role of the individual as a moral agent. This is particularly relevant as it realises the relationship between structures and agents and how this constructs social reality (Bordieu \& Wacquant, 1992).

A number of researchers have acknowledged the restraints of single level exploration of CSR, advocating the multilevel approach (Aguilera et al., 2007; Moore \& Spence, 2006; Spence, 1999). However, studies that actually address this issue are more 
limited and varied in their application (Aguilera et al., 2007; Ibrahim, Jamali., \& Karatas-Ozkan., 2012; Wood, 1991). By adopting the multilevel approach it would challenge assumptions in the current literature by acknowledging how different aspects of the SME context integrate to influence CSR. This framework of analysis is also claimed to help reveal true motivations driving behaviour (Wood, 1991), addressing a key research objective of this thesis to find out the key drivers of CSR. Consequently, whilst this research is exploratory, the different levels of analysis shaping CSR will be acknowledged and addressed as the findings emerge.

\subsection{7: Summary}

The section above provides a summary of literature for CSR in SMEs and outlines central theoretical and analytical approaches to be adopted in this thesis. A number of key observations have also been recognised to inform the subsequent research. The first is the significance of SMEs and their distinct characteristics in comparison to their larger counterparts. These differences result in a disparity of CSR approaches, reinforcing the need for continuation of size specific research and adoption of the small firm perspective into the main body of CSR literature. There is also a further need for theoretical development of the concept. This research will adopt a qualitative methodology in order to confirm and potentially advance specific SME theories in line with this gap in the literature.

A second observation is an attempt to establish the CSR perspective most commonly adopted by SMEs. In line with the main research question, this theme relates to the conceptualisation and motivations driving CSR and the endeavour to establish if CSR is understood as an economically rational or moral construct. There is currently dissensus as to the key motivations for SMEs, although research recommends that there is no point advancing the business case with SMEs. Instead, there are claims that focus should return to the moral foundations of CSR and intrinsic motivations. It would be fair to conclude from the current literature that SMEs are driven by more than economic rationality and profit when engaging with CSR. Therefore, further research is warranted that establishes the motives that drive CSR engagement. 
A third observation is the characterisation of stakeholder relationships. SME stakeholder relationships differ to large firms in a number of ways. Business is inherently personal for SMEs, with activities legitimised by key stakeholders. There is a reliance on informal ties for survival and as a consequence, SMEs are defined by their embeddedness and connections within local communities. However, this often narrows their CSR focus to a community level. Stakeholder theory and social capital theory both appear to be valuable to adequately analyse the importance of stakeholder relationships. Although, there is still a requirement for further, size sensitive developments that adopt these CSR theories.

A fourth reflection is the informal nature of SMEs. This can both enhance and restrict SME's susceptibility to CSR. The informal structure and nature of operations allow freedom for intuition and the ability to be responsive to the context. Yet CSR engagement is often not recognised by SMEs, implemented on a small scale and not strategised or budgeted. Whilst this is largely accountable to the lack of resources, there is increasing external pressure for SMEs to formalise and validate their engagement. This is despite claims that formalisation is not necessary for SMEs to prove their ethical conduct or social engagement. However, the literature does imply a need for better tools to initiate communication of CSR in SMEs, primarily to gain recognition of the valuable social engagement going unnoticed in SMEs.

Finally, the theoretical frameworks adopted to guide the research have been outlined. There is still a need for research that accommodates the unique characteristics of SMEs and acknowledges their contextual nature. With regards to that, a multidimensional framework of analysis mentioned in section 3.3.6 will be adopted to analyse the research. The redrawn stakeholder theory and SBSR pyramids will be used to guide the research and allow for the confirmation or extension of theory. They will also assist with establishing the relevance of the ethic of care and moral proximity for the advancement of CSR research in SMEs.

\section{4: Chapter Conclusion}


To conclude, this chapter established the conceptual foundations of CSR and identified gaps in the mainstream literature that influenced the research. Due to the contextual relevance and significant gap in knowledge, this research focuses specifically on CSR in the SME setting. The chapter offered a number of observations following a review of the existing SME literature, as outlined in the summary above. Most notably was the need for theoretical developments that acknowledge the distinct characteristics, unique CSR perspectives and informal nature of SMEs. The literature revealed the significance of context, recognising limitations in the current theories and methodologies adopted. Therefore, the following study fills this fundamental research gap by empirically evaluating the role of two moral perspectives to theoretically advance the research agenda. In order to address methodological weaknesses, a qualitative methodology and a multi-layered lens of analysis is adopted. The following chapter will now set out the methodological approach and defence of methods in more detail. 


\section{Chapter Four: Research Methodology}

\section{1: Introduction}

The following chapter will show how the research question, methodology and methods are aligned. Consequently, it will begin with an overview of the research approach including the methodological underpinnings focusing on ontology and epistemology and the research design perspective. Following this, the data collection and analysis methods will be justified and research ethics and limitations discussed.

\section{2: Overview of the Research Approach}

The following sub-section provides a summary of the research methodology chapter and overall research design. This is to provide the reader with an appreciation of the overall project prior to immersion in the detailed explanation and justification of the methodology and method (see Figure 4.1).

Figure 4.1: Methods Map

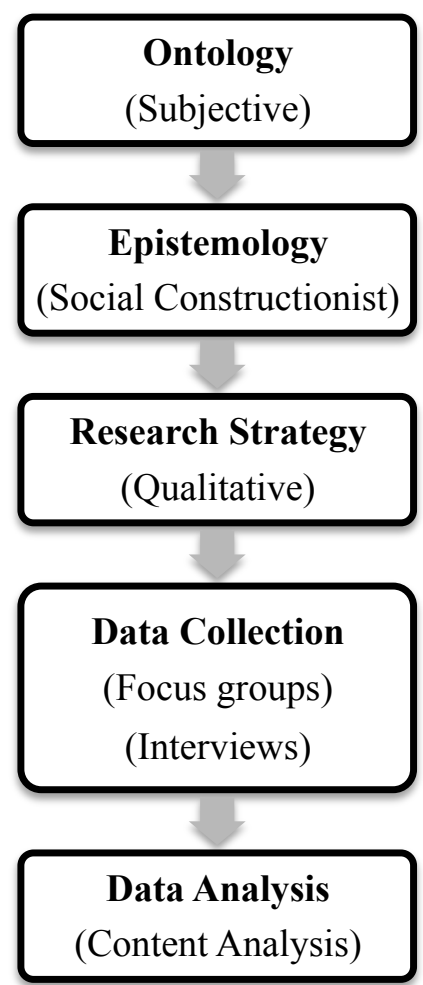


Epistemological position

This thesis is a qualitative study, taking a social constructionist perspective. This position will be explored in more detail below.

Overall Research Strategy

The overall research strategy is qualitative, taking an inductive, interpretive approach to the subject.

\section{Data collection}

The data collection methods consisted of four focus groups followed by thirty semistructured interviews (inclusive of two pilot interviews).

Four focus groups were conducted at the beginning of data collection in order to explore relevant themes to the research questions and allow others to develop and emerge. The four groups contained nine, seven, five and six respondents. They comprised of owner-managers or key decision makers of SMEs.

The semi-structured interviews were then conducted over a period of three months. These were informed by the themes of the focus groups, previous literature and some a priori questions informed by the research propositions at hand. Thirty interviews were conducted with SME owner-managers. The two initial interviews were used as a pilot study, to sense-check the approach being taken. However, the exploratory and formative role played by the focus groups meant that negligible alteration of the interview schedule was required. This means it is appropriate to include the data from these two interviews. The rationale for this approach will be explored in section three of this chapter below.

Data analysis and presentation 
The analysis of the focus group and interview transcripts was conducted using content analysis with coded themes. This was based on the transcription and analysis of voice recordings from both data collection methods. The findings are presented and analysed using verbatim, direct quotations from the texts, interposed with interpretations from the researcher.

Having presented a brief descriptive overview of the research design, the following will provide a more detailed explanation and defence of the methodology and method. The next section will be on the methodological positioning of the thesis.

\section{3: Methodological Underpinnings of the Thesis}

This section introduces the methodological underpinnings of the thesis with regards to the research ontology and epistemology. These encompass the theory of knowledge and our view of reality that underpins the theoretical perspectives of research and overall methodology. The research perspective that has been adopted will be articulated, with this informing the design, data collection and analysis. The next section will intimately inform the following chapters of this thesis.

\subsection{1: Research Ontology}

Ontology deals with "the very nature of "being"” (Marsh \& Furlong, 2002, p. 18) and what we constitute as reality. For example, how can we understand existence and is there "a 'real' world 'out there' that is independent of our knowledge of it" (Marsh \& Furlong, 2002, p. 18). The ontological positioning of this research therefore clarifies what is considered 'real' within this thesis. This research takes the 'construction' view proposed by Potter (1996) whereby "the world is not ready categorized by God or nature in ways that we are all forced to accept" but "reality enters into human practises by way of the categories and descriptions that are part of those practises", "it is constituted in one way or another as people talk it, write it and argue it" (p. 98). 
This is not to argue that the natural sciences or the world as a whole is constructed incrementally through our interactions and actions. It is appreciated that there are phenomena independent of human opinion. What is being suggested is that language and human practices are under continuous construction; therefore it is not possible for participants to articulate a reality that is not affected by their construction and interpretation of that reality. Sensemaking is undertaken by questioning the social and historical roots of phenomena. As 'The Thomas Theorum' explains, "if a person perceives a situation as real, it is real in its consequences" (Thomas \& Thomas, 1928, pp. 571-572). This view therefore considers the world as being socially constructed.

\subsection{2: Research Epistemology}

Whilst ontology has already been discussed as the nature of being and representation of reality, epistemology is the relationship between reality and the researcher and the best ways to discover valid knowledge about the world (Carson, Gilmore, Perry \& Gronhaug, 2001). It "is concerned with providing a philosophical grounding for deciding what kinds of knowledge are possible and how we can ensure that they are both adequate and legitimate" (Maynard, 1994, p. 10). As Crotty (1998) explains, ontology sits alongside the epistemology to help inform the theoretical perspective taken and guide the overall research design. There are two common approaches to epistemology, positivism and interpretivism (Carson et al., 2001; Wheeldon \& Ahlberg, 2012)

Positivism seeks to "explain and predict what happens in the social world by searching for regularities and casual relationships between its constituent elements" (Burrell \& Morgan, 1979, p. 5). This allows for objective analysis and views knowledge as independent of people (Girod-Séville \& Perret, 2001; Levin, 1998). This perspective is dominant in the natural sciences and proposes a single objective reality to any research situation (Hudson \& Ozanne, 1988), regardless of the human perspective of the researcher.

Interpretivism on the other hand is "an epistemological notion asserting that meaning exists in objects independently of any consciousness" (Crotty, 1998, p. 10). It 
considers the social world to be relativistic, only to be "understood from the point of view of the individuals who are directly involved in the activities which are to be studied" (Burrell \& Morgan, 1979, p. 5). This maintains a subjective and socially constructed view of knowledge (Berger \& Luckman, 1966) and means that research is affected by the interpretation of both participants and researcher. Due to the complex nature of 'reality', flexible research structures are favoured to capture meaning in human interaction (Carson et al., 2001). The goal of the researcher is to allow knowledge to develop throughout the process, aiming to interpret and understand time bound, subjective experiences (Hudson \& Ozanne, 1988). The key differentials of a positivist and interpretivist paradigm can be seen in Table 4.1 below.

Table 4.1: Key Differentials of a Positivist and Interpretivist Paradigm (Adopted from Carson et al., 2001, p. 6)

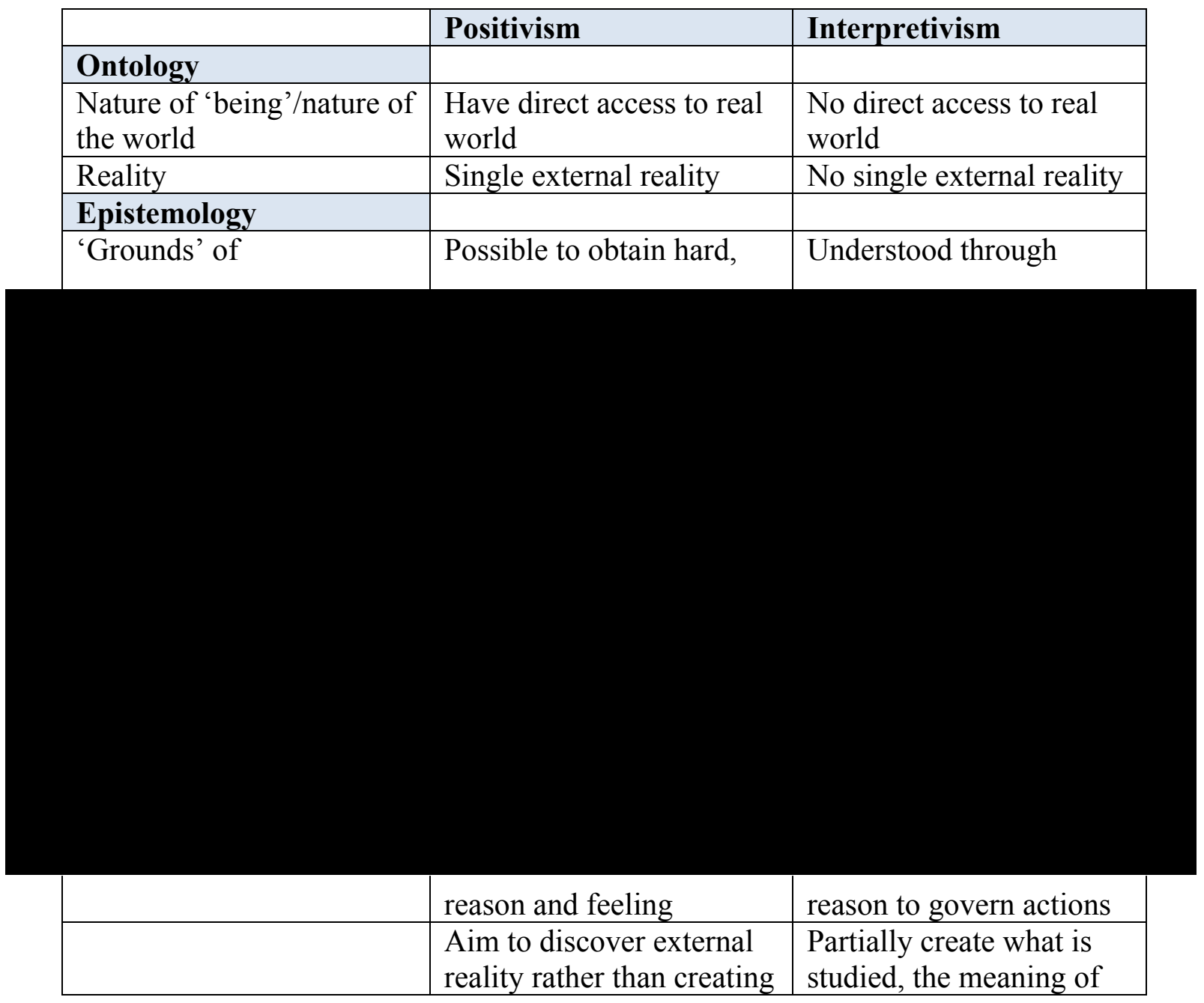




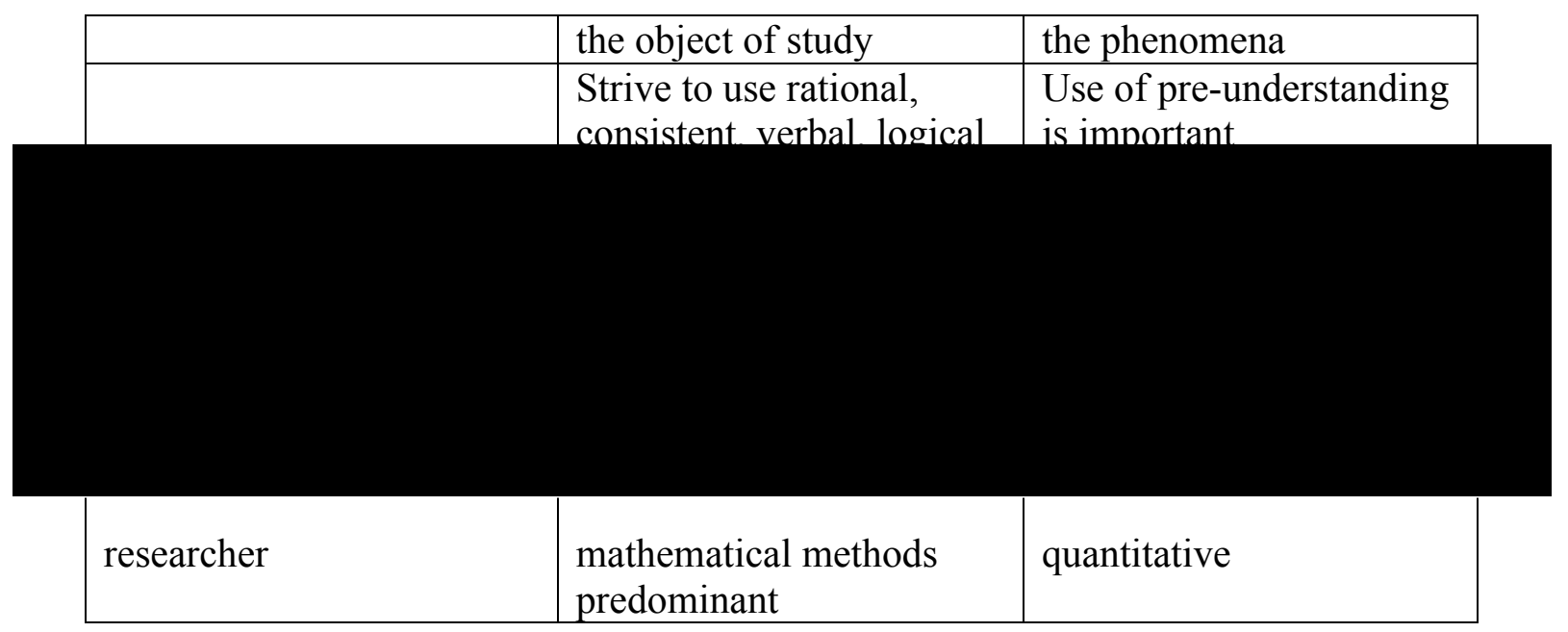

Having distinguished the key differentials of ontological and epistemological stances, this research can be positioned within the interpretivist paradigm. This is because it is exploratory and deals with participants in focus group and interview settings whereby the priority is for natural discussion. This is in order to derive themes in the respondent's own language (Gamson, 1992) and draw from the interpretive repertoires of a group of participants close to the subject area (Potter, 1996). Consequently, it is not possible to separate the actors from that particular setting and relationships formed previously or within it.

Therefore, it is important to acknowledge that the research approach recognises that the knowledge from this study is affected by the social context. The research methods chosen allow for interaction between participants and the researcher in order to establish how SME owner-managers conceptualise CSR. The researcher as a facilitator of the research can analyse and interpret findings based upon what is found in the discourse and observed as reality during the data collection. As such, this research also follows a social constructionist approach. Social constructionism as a research perspective will be discussed in more detail below.

Epistemologically this research attempts to understand how SME owner-managers attach meaning to CSR. The research design takes the interpretivist stance because the researcher is part of the research setting and their presence and interpretation of the findings partially creates the understanding of the phenomena of CSR in SMEs. Furthermore, participants were specifically chosen as a sample of SME owner- 
managers, with the aim of understanding the appropriateness of returning theories to the 'moral' for CSR in SMEs.

\section{4: Research Perspective: Social Constructionism}

Berger and Luckmann first introduced the term 'social construction' in 1966 in their book The Social Construction of Reality. Their central concept is of multiple realities and the acknowledgement that reality is socially constructed through the incremental acquisition of knowledge in our interactions. This could be considered more of a 'nominalist' position in what Burrell and Morgan (1979) describe as 'the ontological debate'. This is whereby the ontological position "revolves around the assumption that the social world external to individual cognition is made up of nothing more than names, concepts and labels which are used to structure reality" (Burrell \& Morgan, 1979, p. 4).

Due to the contextual nature of SMEs and the multiple realities of individuals, this study requires in-depth exploration of SME owner-managers to discover what drives them to be socially responsible. A broadly inductive strategy and social constructionism research perspective is considered appropriate to do this. This locates the methodological position of this research and will later inform the methods used for data collection.

Accepting the role as a social constructionist requires taking on certain key assumptions within the research perspective. Burr (2003) proposes that there are four fundamental assumptions that should be adopted. The first of those is taking a critical stance towards taken-for-granted knowledge. Social construction can be seen as a critique of positivist science and "insists that we take a critical stance towards taken-for-granted ways of understanding the world and ourselves" (Burr, 2015 , p. 2). The second is the understanding of historical and cultural specificity, the third is the acceptance that social processes sustain knowledge and the final is that knowledge and social action go together and are bound by power relations. 
In the context of this thesis in terms of the epistemological position, social constructionism locates the research methodologically. This informs the data collection methods and analysis. On the other hand, CSR as the main phenomenon being studied can also be socially constructed from an ontological perspective. For example, owner-manager's understanding of CSR is developed through the acquisition of knowledge in their social interactions. It is constructed as a concept incrementally within their reality and sustained through the language used in social processes. This is particularly relevant in SMEs due to the lack of consistent language for the concept of CSR. The way in which SME owner-managers make sense of the term is also historically and culturally relative and can be considered an artefact of that time which has and will change temporally.

\subsection{1: Co-construction}

Having discussed the key assumptions for a social constructionist study, the next area that needs to be acknowledged is the researcher as a co-constructor of knowledge and their perception of the participant's 'reality'. This further affects what is considered as 'real' and results in a double hermeneutic as described by Marsh and Furlong (2002) and Giddens (1987). This is that "no observer can be 'objective' because they live in the social world and are affected by the social constructions of "reality"” (Marsh \& Furlong, 2002, p. 19). For example, "the world is interpreted by the actors (one hermeneutic level) and their interpretation by the observer (a second hermeneutic level)" (p. 19) and therefore the assumptions in our language and theories can both reflect and also create reality. Despite trying to remain aware of the research perspective throughout the study, it is also important to recognise the researcher's role in interpreting and constructing the discourse during the data collection and analysis.

As Burr explains, the place of the researcher is to:

\footnotetext{
"...Acknowledge and even work with their own intrinsic involvement in the research process and the part that this plays in the results that are produced. The researcher must view the research as necessarily a co-production between themselves and the people they are researching." (2003, p. 152)
} 
For example, when applied to the language used during the focus groups and interviews, it is probable that the intrinsic involvement of the researcher plays a part in contributing to the participants understanding. The a priori assumptions used to guide the initial research play a part in how participants understand the topics during discussion and ultimately how they interpret CSR.

Having set out the ontological and epistemological position of this research, the research perspective as social constructionist and acknowledged the researcher as a co-constructor of reality, the following section will turn to a more in-depth positioning. This will be done with regards to Deetz's $(1994,1996)$ development of the four-paradigm model for the analysis of social theory (Burrell \& Morgan, 1979).

\section{5: Positioning the Research}

Burrell and Morgan (1979) have discussed the underpinning assumptions that are thought to inform research: ontology, epistemology, human nature and methodology. This research led to the development of their four-paradigm model for the analysis of social theory. Albeit widely cited, Deetz $(1994,1996)$ critiqued and developed the model claiming that rather than being used to provide attention to differences in research programs; it has often been used as a classification of four 'things'. Deetz (1996) proposed that there was a need to stop categorising and defining and rather focus on the dimensions of contrast and "the lines of distinction producing some things as alike and others as different” (p. 192).

The positioning of this thesis will therefore be explained using the two dimensions proposed by Deetz $(1994,1996)$ and further developed by Alvesson and Deetz (2000). The dimensions mentioned consist of 'consensus-dissensus seeking' in respect of research practises in social discourses and 'local/emergent-elite/a priori conceptions' regarding the origins of concepts as part of the process in research. Both of these will be discussed in greater detail in order to explain how they are specifically relevant when applied to the positioning of this research. 
Figure 4.2: Contrasting Dimensions from the Metatheory of Representational Practises. Reproduced from Alvesson and Deetz (2000). Adopted from Deetz (1994).

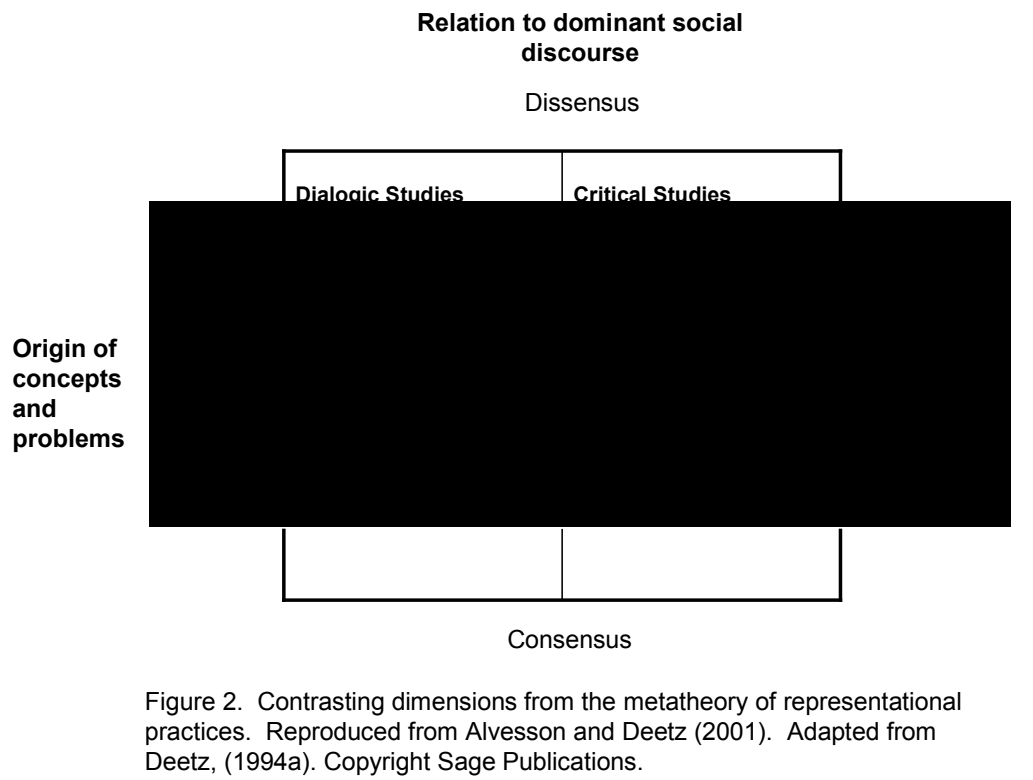

\subsubsection{Consensus to Dissensus}

The dimension of consensus-dissensus highlights the integral relationship of research to existing social orders and importantly the limitations of dominant discourses. Such dominant discourses are most often seen in the micro-practises of the business itself, but also at the macro-sociological on a smaller scale (Knights \& Willmott, 1989). This is a problem due to the "suppression of parts of the human being and the presence of destructive control processes" (Alvesson \& Deetz, 2000, p. 25) and is of particular relevance in this thesis as the concern is returning back to the moral aspect of CSR in the everyday micro-practises of SMEs. By doing this it is challenging the dominant CSR discourse that emphasises the economic superseding any kind of moral implications.

The primary concern of the consensus pole of this dimension is to "display a discovered order with a high degree of fidelity or verisimilitude" (Alvesson \& Deetz, 2000, p. 26). This means that such research aims to 'mirror' behaviours and relationships that exist in the external world, or in terms of discourse those that 
dominate. This uncontested 'reality' is not seen as problematic and language "is treated as a system of representations, to be neutralized and made transparent" ( $p$. 26). Consequently, the research does not seek to challenge the dominant discourses, but to seek consensus.

Alternatively, the dissensus pole of this dimension takes form of a 'lens'. This regards research that aims to reveal what could not be seen before to the undisputed 'mirror'. It acknowledges "the researcher as positioned and active" (Alvesson \& Deetz, 2000, p. 26), recognising the context rather than attempting to neutralise them. The dissensus pole does not aim to disregard the importance of an ordered world and dominant discourses, but rather to challenge "assumptions, values, social practises and routines" (p. 26). This identification of the world and identity as conflictual, in process and under continuous construction leads to diverse avenues for fuller potential. It also makes clear the complexities and variety in such dominant discourses. This resonates with the interpretivist ontological position adopted in this thesis.

\subsection{2: Local/Emergent to Elite/A Priori}

The dimension of local/emergent to elite/a priori is concerned with the origins of research concepts. For example, where and how did the concept begin and in what context? When applied to CSR, is the concept developed incrementally during the research process with the concept emerging through interactions with participants? Or was it applied to the research as a pre-conceived concept that was held 'static' throughout?

The elite/a priori pole of this dimension draws attention to research where the language system of the researcher is used and constant throughout the process. It also privileges their knowledge and the research community they are part of. The extreme of this spectrum demands definitions to be established and accepted prior to the research starting and alterations in the conceptual system to happen outside of the research process. It follows the view of essentialism and rationality, that everything has characteristics prior to existence that make them what they are. For example, 
trust in existing narratives and the emphasis on positioning findings in existing theories are dominant.

On the other end of the scale, language used for the local/emergent is open and constantly being adapted. The theoretical vocabulary used and knowledge claimed is less fixed and focussed on insights rather than truth. Adaptations are welcomed based on participant interaction in the research process and cumulative understanding is developed through "stories or accounts" and attending to "feelings, intuitions and multiple forms of rationality" (Alvesson \& Deetz, 2000, 29) in the research enterprise. This is alternate to one, purified form of rationality and objectivity. Therefore, local/emergent research is aimed at the gradual formation of ideas and the acceptance of the researcher as a co-constructor of knowledge.

\subsection{3: Epistemological Position of the Research}

In order to facilitate a more detailed consideration of the epistemological position of this research, the above dimensions are referred to. However, rather than situating the research at a static point, it may be more appropriate to discuss the various points that have been located at different times during the research process. At the initial stages, the researcher held a simplistic view of CSR and referred to dominant discourses. The reference to two core CSR theories provided stability in linguistics and two clear concepts, with the expectation that the data would display truth in these narratives and position the research as elite/a priori.

However, as the literature search and focus group findings emerged, it became obvious that CSR was complex and constructed incrementally as a form of 'practical' knowledge. Participants provided scattered interpretations of the concept, with their "interpretive repertoires" (Potter \& Wetherell, 1987, p. 138) drawing from individual accounts and contextual reflections that were influenced by numerous stakeholders. There was no particular reference to terms adopted within the academic literature and the findings disrupted the current discourse by suggesting that CSR is constructed as a moral or relational concept, rather than a means of organisational success. 
This disregarded the option of holding any concepts static and enabled the research to be situated in the 'local/emergent' side. The aim of this study was not to 'mirror' or seek consensus of undisputed dominant discourses, therefore the complexities were accepted rather than 'neutralised'. Consequently, at this point of the research the dissensus view was preferential. The researcher sought to understand tensions in the way that participants constructed and enacted CSR within their businesses. Issues of voice, power and proximity were integral to understand the way that meaning was incrementally constructed around CSR (Jones, 1991; Munro, 2001). Therefore, the constant re-differentiation of experience and identity of participants was acknowledged and appreciated.

However, as the data collection developed and themes began to arise out of the analysis, it was difficult to not look for similarities offered by respondents. Commonalities were arising in the language adopted and justifications for engagement. In fact, the semi-structured interview questions were drawn from common themes, patterns and concepts that arose throughout the focus groups. Therefore, during the interview design the research could be considered to return to a more elite/a priori position.

To account for this, actions were taken to return to the local/emergent. Thematic questions were formed that moved from general enquiry to the more specific. This was to ensure an untarnished representation of the construction of CSR that did not influence the respondent's answers with thoughts from the dominant discourse. Furthermore, the researcher made a conscious effort to acknowledge their position as a co-constructor of knowledge and the tendency to draw from their own reality. Instead, it was recognised that their role was to simply guide, observe and listen to respondents.

Having explored both dimensions, plotting a single position on the matrix would not be useful or explanatory. At the early stages of research a normative and modern approach was adopted on the matrix. This moved quickly to hover over the dialogic during the exploratory focus groups and back to dissensus and the critical realm for the construction of the interview themes. The position here then oscillated between a more dialogic position and the critical realm during interview construction and data 
collection (see Figure 4.3). From this analysis of the matrix, it is possible to consider that the labelling of one single position is not mandatory and can be accommodated without creating undue tensions in the epistemological position of this thesis. As Alvesson and Deetz (2000) explain, "important insights can be gained by actively pursuing a reproachment between critical and interpretive work" (p. 12).

Figure 4.3: Research Position on the Contrasting Dimensions from the Metatheory of Representational Practises. Reproduced from Alvesson and Deetz (2000). Adopted from Deetz (1994).

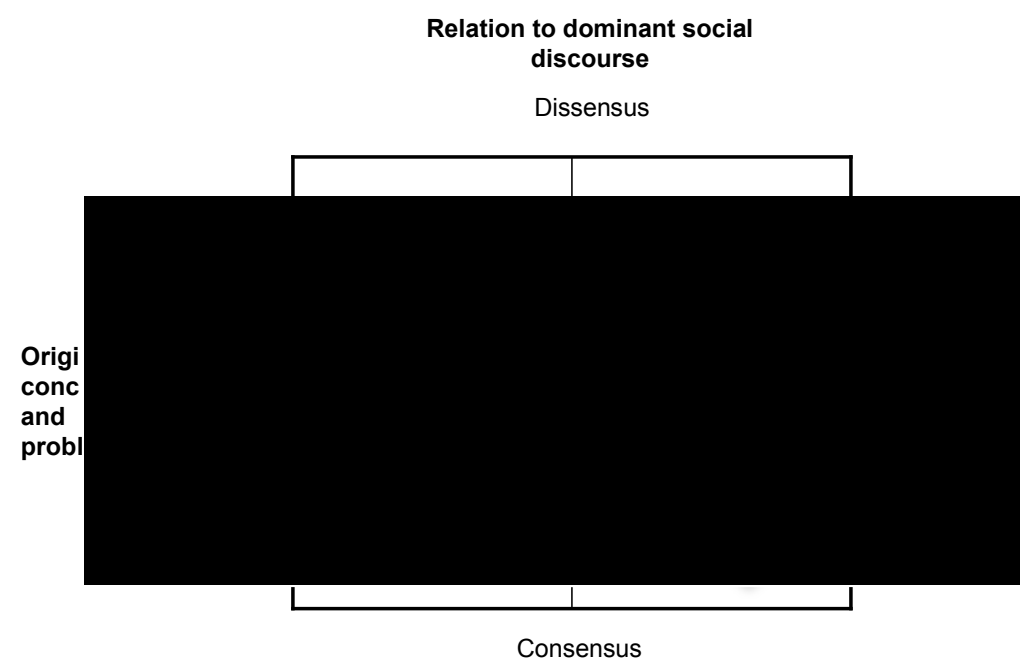

Figure 2. Contrasting dimensions from the metatheory of representational practices. Reproduced from Alvesson and Deetz (2001). Adapted from Deetz, (1994a). Copyright Sage Publications.

The past two sections have outlined the epistemological position and research perspective adopted. The next section will explain and outline the data collection methods and the form of analysis used.

\subsection{Methods: Data collection}

\subsection{1: Introduction}

The overall research strategy adopted for this study is qualitative, informed by the methodological position discussed above. Due to the research being interpretivist and inductive in nature, the methods should therefore reflect this. An interpretivist 
methodology is commonly associated with data collection through qualitative methods such as ethnography, participant observation, focus groups and interviews (Bryman \& Bell, 2011). In this case there were two phases of data collection, therefore the methods chosen for this research included:

1. Focus groups

2. Semi-structured, in-depth interviews

The focus groups were exploratory and utilised to gain an appreciation of SME owner-managers' understanding of 'CSR' and to inform the research questions by deriving emergent themes. There were four focus groups with nine, seven, five and six participants in the different regions of Wales. The focus groups were followed by thirty semi-structured, in-depth interviews with SME owner-managers.

The following section will explain and justify the data collection methods and analyses that were used, aligned and adapted during the development of this research. It will include a discussion on the protocols, sampling and coding and validation for the use of a pilot study, focus groups and interviews. Finally, there will be a justification for the themes and questions used within the study and reflection on the socially desired response syndrome, validity, reliability, research limitations and ethical considerations.

\section{7: Data Collection Method: Focus Groups}

The first phase of data collection was that of focus groups. A focus group can be described as "an interview style designed for small groups, typically involving from six to ten people discussing and commenting on particular topics or concepts under the guidance of a moderator" (Blackburn \& Stokes, 2000, p. 45). Focus groups have "very elastic boundaries" (Morgan, 1988, p. 35) and can be used at many stages of the research process (Hansen, Cottle, Negrine \& Newbold, 1998). However, there are numerous justifications for their use as an exploratory method at the initial stages of this study. 
The primary goal of the focus groups was to "generate conversations that uncover individual opinions regarding a particular issue" (Cyr, 2016, pp. 233-234) and reveal group consensus if and when it exists regarding the topic at hand. This elicits a range of experiences and perspectives (Cyr, 2016) that establish conceptual boundaries or 'unit of analysis' for CSR (Baden et al., 2011; Miles \& Huberman, 1994). It also allows information to be drawn from the interpretive repertoires of a group of participants close to the subject area (Gamson, 1992; Potter, 1996), uncovering key themes in the respondent's own language.

Focus groups are unique as they allow a shift of power from researcher to the researched, enabling respondents to debate why views are held (Blacburn \& Stokes, 2000; Cyr, 2016) and modify or qualify accordingly to provide a more realistic account (Bryman \& Bell, 2011; Lancaster, 2005). This has been found to be true for SME owner-managers where they "empathise, explain, challenge, contradict and advise on, each other's accounts of their experiences" (Blackburn \& Stokes, 2000, p. 61 ), providing a platform for each other to contribute. This is known as a synergistic group effect (Stewert \& Shamdasani, 1990) and is facilitated by "feelings of security and spontaneity generated by their interactions" (Blackburn \& Stokes, 2000, p. 46). It also provides an open perspective, useful to establish how respondents make sense and attach meaning to concepts.

Scoping of the topic in the early stages of research is particularly useful to obtain a broad understanding of the research subject and provide "data and insights that would be less accessible without the interaction found in a group" (Morgan, 1988, p. 12). The establishing of conceptual boundaries for CSR and identification of key themes was integral to the research journey. This is because the ultimate themes derived from these exploratory focus groups were used to inform and shape the subsequent interviews.

\subsubsection{Data Collection Process: Focus groups}

\subsubsection{1: Number of Focus Groups}


Between May 2016 and August 2016 four focus groups were arranged to integrate with FSB regional meetings. These meetings were spread geographically around Wales in four different FSB regions in North, South and Mid Wales. Figure 4.4 below shows the geographical distribution of the focus groups and Appendix 4.1 identifies the FSB regional meetings that the focus groups were part of, with the location and date specified.

\section{Figure 4.4: Focus Groups: The Geographical Distribution}

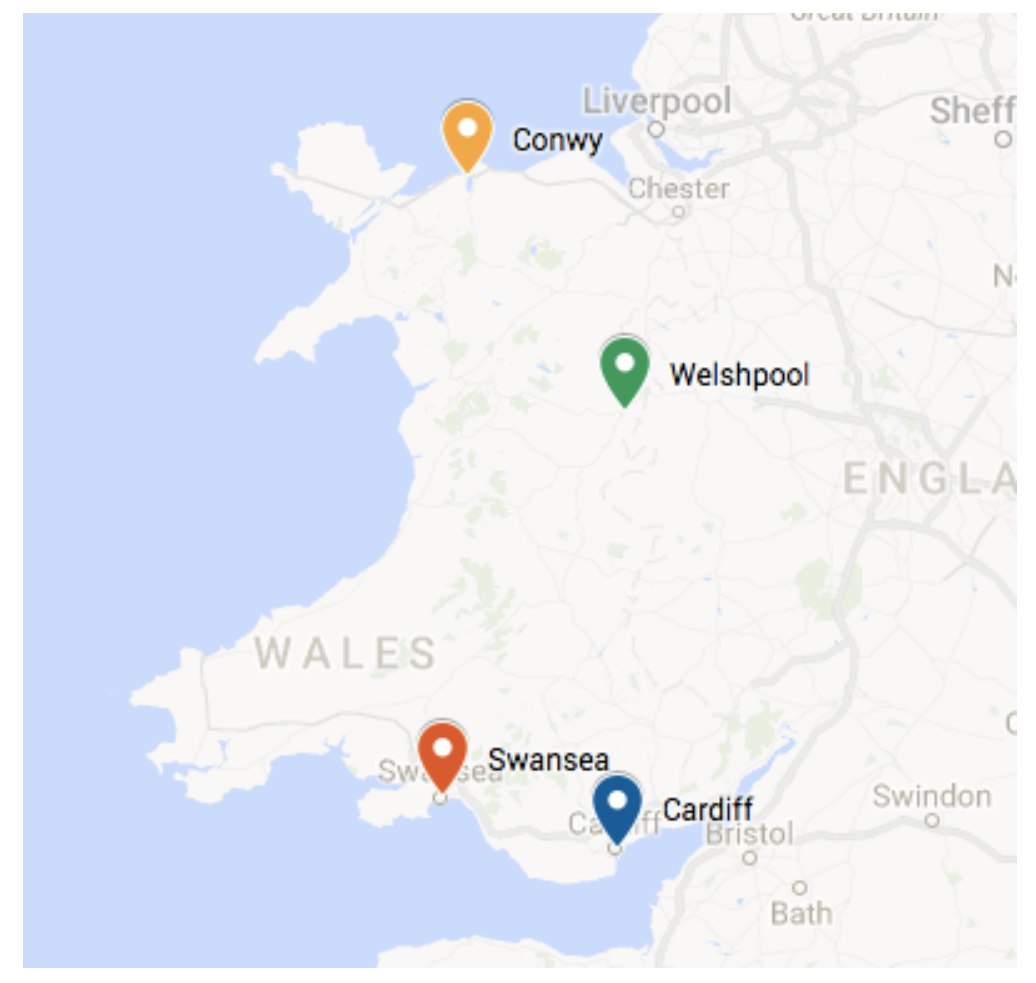

\subsubsection{2: Selection of Participants}

The focus groups were sampled from the FSB membership. The FSB is a non-profit making, non-party political organisation that is led by its members. Their mission is to help smaller businesses achieve their ambitions (The Federation of Small Businesses, 2017) by campaigning to deliver change to support growth and success. With a member base of well over 100,000 in the UK, they have a large national presence and a database inclusive of SME owner-managers. It is from this database of members that the focus group participants came from. 
The FSB worked collaboratively during this initial stage of data collection and the focus groups were integrated into four regional meetings geographically spread around Wales (see Figure 4.4). Participants were all SME owner-managers conforming to the European definition, or key decision makers of the SME (European Commission, 2017). Owner-managers were used because of their central position in the business and role of legitimate decision maker (Jenkins, 2004; Spence, 2016). For the first focus group all FSB members from the Location 1 regional meeting participated in the focus group, whereas with the following three the FSB Development Officer approached members of the relevant region via email with an Eventbrite link to sign up to the focus group.

Thirty-five attended the focus groups with twenty-seven fitting the participant requirements. They consisted of nine participants in Location 1, seven respondents in Location 2, five in Location 3 and six in Location 4, a total of twenty-seven overall in addition to those facilitating the focus groups (see Appendix 4.2). According to Bryman and Bell (2011) a focus group should have at least four participants with a guideline of ten accepted as a maximum number for effective discussions (Blackburn \& Stokes, 2000; Saunders, Lewis \& Thornhill, 2003; Rubin $\&$ Rubin, 2004). The researcher conducted the focus groups with one or two added facilitators from the FSB, this was so that they could take notes and observations as recommended by Saunders et al. (2003). Participants came from an array of different sized SMEs and industries in Wales, as can be seen in the SME profiles in Appendix 4.3 and Table 4.2 below. For the percentage of micro, small and mediumsized businesses represented in the focus groups refer to Appendix 4.4.

\section{Table 4.2: Focus Group SME Sectors}

\begin{tabular}{|l|l|}
\hline Sector & Percentage \\
\hline Information and communication technology & $7.4 \%$ \\
\hline Arts, entertainment and recreation & $7.4 \%$ \\
\hline Accommodation and food service activities & $18.5 \%$ \\
\hline Professional, scientific and technical activities & $14.8 \%$ \\
\hline Financial and insurance activities & $3.7 \%$ \\
\hline Education & $11.1 \%$ \\
\hline Engineering & $3.7 \%$ \\
\hline
\end{tabular}




\begin{tabular}{|l|l|}
\hline Construction & $3.7 \%$ \\
\hline Energy and environment & $3.7 \%$ \\
\hline Wholesale and retail trade & $14.8 \%$ \\
\hline Other service activities & $3.7 \%$ \\
\hline Social enterprise & $7.4 \%$ \\
\hline
\end{tabular}

\subsection{2: Focus Group Research Protocol}

\subsubsection{1: Structured Tasks}

The focus groups consisted of one initial structured section inclusive of two activityorientated activities, with a subsequent open discussion. The main section of the focus groups was the open discussion, however as a preliminary task participants were asked to take part in two activity-orientated tasks that were included in their participant booklet (see Appendix 4.5). This was used as a way of engaging participants and to initiate focus on the core topic of study (Colucci, 2007).

The first activity focused on the theme of key stakeholders and the question below required participants to write down their key stakeholders in order of how important they are to their business. They were then asked to write them down as a free list in the focus group booklet (see Appendix 4.5).

"Who are your main stakeholders? List up to 10 stakeholders in order of how important you think they are to your business. A stakeholder can be any group or individual that interacts with your business."

The second task involved participants ranking four components of SBSR according to Spence's (2016) theoretical developments, in order of how important participants felt they were to their business. As mentioned, these were included to get participants to engage in the topic of discussion, but also to gauge the empirical appropriateness of the recent research. The question below was included in the participant booklet including four definitions, one for each type of responsibility and a list of 1-4 so that participants could rank them in order of priority. The question below was asked. However, the full participant booklet can be seen in Appendix 4.5 . 
"Some recent exciting research into small business social responsibility has split the priorities of small businesses into four different areas."

"We would like you to rank these in order of how important you feel they are for YOUR business (as opposed to businesses in general). There is no 'right and wrong' here - please simply order them according to the way you feel for your business."

\subsubsection{2: Open Discussion Themes}

Following these tasks, the main section of the focus groups was the open discussion that was conducted immediately following the activity-orientated tasks. The use of these initial tasks was to engage participants and get them thinking about who and what responsibilities they prioritise as owner-managers. Discussion was prompted by asking participants to introduce themselves and their business, following this, open conversation was initiated by asking which stakeholders they had prioritised in Task 1 and on what basis they had prioritised them. After this, the following themes from the literature were used as broad guidelines of discussion (see Appendix 4.6). However, these were used simply as a guide with the emphasis instead being on the naturally occurring group discussion.

\section{Stakeholders}

i) Prioritisation

ii) Relationships

\section{Corporate social responsibility}

i) Understanding of CSR

ii) Engagement

iii) CSR terminology

iv) Drivers

v) Barriers

vi) Budget 


\subsection{3: Focus Groups: Role of the Researcher}

Despite wanting an open discussion with participants from the focus groups, the role of the facilitator was to guide the discussion. As Merton, Fiske and Kendall (1990) explained, group dynamics can present problems at times creating a 'group effect'. This could be one group member or a small group dominating proceedings, the reluctance of some participants to speak at all, the need to ensure the whole group has an opportunity to give their views or the risk of conversation diverging from the topic of interest. The facilitator of the focus groups therefore requires certain skills to counteract such group effects. For example, in Location 1 and 4 there were two dominant characters that could have potentially dominated proceedings with their views on particular perspectives of CSR. The researcher therefore intervened from time to time in order to re-direct the conversation back on track and to solicit the views of quieter participants. In this case the facilitators tried to stay as unobtrusive as possible, whilst subtly guiding the direction of conversation if it turned in unhelpful directions (Bryman \& Bell, 2011). Furthermore, at the end of the focus group every individual present was given a chance to make any final points or summarise their thoughts on the topic of CSR in SMEs.

\section{8: Data Collection Method: Interviews}

The purpose of this research was to gain greater understanding of the underlying processes of CSR in SMEs and the way that SME owner-managers attach meaning to the phenomenon. The in-depth, semi-structured interview was chosen due to the appropriateness to both the epistemological position and research perspective. For example, interviewing is seen as an appropriate method of qualitative enquiry to obtain descriptive data on social construction processes, ethics, human interaction and insights into the values, views, perceptions and actions of organisational members (Cornelissen, 2017; Easterby-Smith, Thorpe, \& Lowe, 2002; Rubin \& Rubin, 2004). As Liedtka (1992) argues, the personal interview is particularly suitable for complex and exploratory research in the social sciences and topics such as ethical decision-making or CSR in this case. It is also congruent with the 
thematic content analysis conducted on the focus group findings, as these themes were used to inform the interview questions.

An in-depth, semi-structured interview can be described as an interview with a guide of questions to be answered, but with flexibility as to how the participant may reply. This is appropriate because themes had already been established from the content analysis of the focus groups to guide the interview content. There is no formal order in a semi-structured interview and the number and wording of questions can change if the interest shifts or becomes more focused. This leaves scope for additional questions to be added or left out of the interview plan where appropriate (Bryman \& Bell, 2011).

The use of in-depth, semi-structured interviews was to provide a research atmosphere that nurtures a conversational style interview. This allows the voice and perspective of the respondents to be heard. It also allowed the researcher to gain an understanding of the "intricacies of the setting" (Cornelissen, 2017, p. 372) to establish how owner-managers make sense of CSR. The semi-structured element then allows the interview themes and responses to be aligned with the research interests from the focus groups.

Further advantages of semi-structured interviews include the collection of rich and descriptive data aligned with the focus group themes (Curran \& Blackburn, 2001), as well as the flexibility to avoid "pre-emptive reduction of the data that might have limited discovery or eliminated complexity" (Campin et al., 2013, p. 497). This is considerably important to develop an understanding of a complex phenomenon such as CSR and is the reason why a number of studies on this topic have utilised this data collection method (Baden et al., 2011; Campin et al., 2013; Davies \& Crane, 2010; Jenkins, 2006; Tilley, 2000).

\subsection{1: Data Collection Process: Interviews}

\subsubsection{1: Number and Date of Interviews}


The semi-structured interviews were carried out between December 2016 and March 2017. In total thirty interviews were conducted inclusive of two pilot interviews, with the participants consisting of owner-managers of SMEs. Again, what constitutes an SME conformed to the official European definition to remain consistent with the research context and focus groups (see Table 3.2). Consequently, the population of the study is any owner-manager of a business that is included in the SME category.

\subsubsection{2: Selection of Participants}

The interviews were predominantly based in and around South Wales. The techniques utilised are visualised via Figure 4.5 below, applying convenience and snowball sampling.

Figure 4.5: Criteria and Steps for Selecting the Research Sample

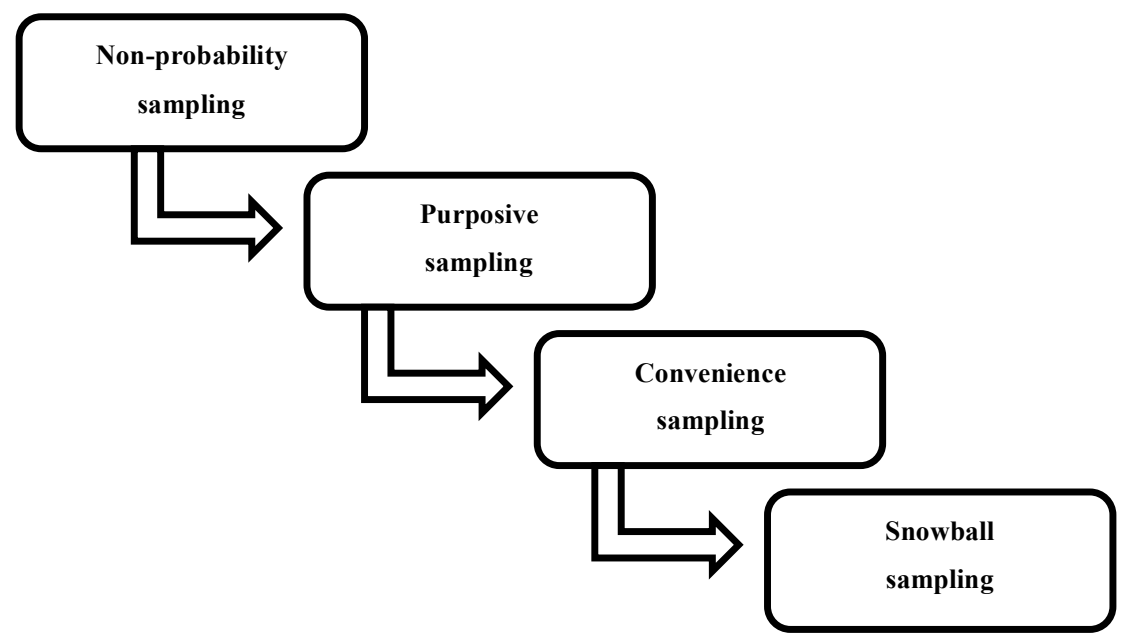

The interviewing process started with the establishment of appropriate participants to represent SMEs. Owner-managers were consequently contacted direct via email (see Appendix 4.7) using a non-probability, purposive method called convenience sampling. This can be described as a sample that is "simply available to the researcher by virtue of its accessibility" (Bryman \& Bell, 2011, p. 190) which was complimented by further snowball sampling. However, despite convenience and snowball sampling, a range of micro, small and medium-sized businesses participated in the research along with an array of sectors (see Appendix 4.8). 
Measures were also taken to ensure that there was a selection of SMEs that operate online or as virtual organisations. Table 4.3 shows the representation and diversity of sectors, with detailed SME profiles available in Appendix 4.8. For the distribution of micro, small and medium-sized businesses represented in the interviews refer to Appendix 4.9.

There are similar justifications to the focus groups for continued use of ownermanagers of SMEs. The first of these is their integral and consistent role as the key decision-makers in the business (Jenkins, 2004; Spence, 2016; Spence \& Rutherfoord, 2001). Consequently, they are usually in a position to present an accurate overview of the SME. Furthermore, one of the key themes permeating discussions in the focus groups was the role of personal values and the need for individual responsibility. The complexity apparent from the initial stages of data collection prompted continued exploration using owner-managers as the unit of analysis.

Table 4.3: Interview SME Sectors

\begin{tabular}{|l|l|}
\hline Sector & Percentage \\
\hline Information and communication technology & $10 \%$ \\
\hline Arts, entertainment and recreation & $26.6 \%$ \\
\hline Accommodation and food service activities & $6.7 \%$ \\
\hline Professional, scientific and technical activities & $10 \%$ \\
\hline Financial and insurance activities & $3.3 \%$ \\
\hline Education & $6.7 \%$ \\
\hline Engineering & $6.7 \%$ \\
\hline Construction & $3.3 \%$ \\
\hline Energy and environment & $0 \%$ \\
\hline Wholesale and retail trade & $16.7 \%$ \\
\hline Other service activities & $6.7 \%$ \\
\hline Social enterprise & $3.3 \%$ \\
\hline
\end{tabular}

\subsection{2: Interview Research Protocol}

\subsubsection{1: Pilot Study}


The piloting process used for this study was inclusive of the focus groups and two initial pilot interviews. The focus groups are included in this process as they allowed a refinement of main themes to which the interviews were then structured. The emergent research strategy also allowed the flexibility to ask additional questions or address issues in subsequent interviews if required. That being said, the inclusion of two pilot interviews was considered necessary and important to trial the interview schedule and assess if it was feasible for SME owner-managers (Bryman \& Bell, 2011; Holloway, 1997). They further check the validity of the focus group data.

The two initial interviews were used as a pilot in order to:

- Gauge the appropriateness of questions

- Highlight any ambiguous questions

- Establish if certain questions were repeated

- Establish if wording used was suitable

- Highlight if the question structure was logical

- Indicate the interview length

- Allow the researcher to determine the adequacy of instructions to interviewers

- Enable experience of conducting semi-structured interviews

- Refine interviewing technique

As recommended by Oppenheim (2000), the use of a pilot study as part of the research was valuable for a number of reasons. The emergent themes from the focus groups represented a pragmatic structure to approach the interviews. Furthermore, by utilising the two initial interviews as a pilot, it sense-checked the approach and revealed a number of issues to be aware of and to amend for proceeding interviews. For example, there were a number of questions that prompted similar responses and the use of certain words were omitted due to ambiguity from respondents, resulting in the rephrasing of some questions.

The structure of the questions asked was also amended for fluidity, for example the questions asking to prioritise responsibility were better grouped together rather than 
thematically. However, due to the planned semi-structured nature of the interviews it allowed the flexibility to make these alterations with regards to the number and wording of questions. Consequently, despite a number of outcomes from the pilot studies, only minor alterations of the interview schedule were required. This was partly due to the emergent nature of the research and formative role of the focus groups that had already established the research landscape preceding this. It also meant that it was appropriate to include the transcribed data of the two interviews within the main interview data analysis.

\subsubsection{Interview Structure}

Due to the semi-structured nature of the interviews, the length and process of each interview varied considerably depending on the participant. The interview was deliberately conducted in a way that sought to avoid introducing the explicit use of the term 'CSR' until the latter stages of the interview. This sustained the exploratory nature of the research initially; with open questions further aiding the exploration of the topic without directing the discussion there. The first question and overall theme to start interview proceedings was open in order to get a feel for initial topics associated with social responsibility and the general character of the SME in terms of the way they view their economic and social responsibilities:

\section{Main theme: \\ As a small or medium-sized enterprise (SME), how do you prioritise your responsibilities?}

As can be seen, the term CSR was not used and instead participants are asked about their responsibilities in general. This provides scope to see what SME ownermanagers automatically think and associate with in terms of their priorities. A number of themes then followed this question in order to structure the interview questions, these can be seen below. However, to reiterate, these acted as prompts during the interview process. Under each theme there were then more detailed inquisitions, but again this was not with the intention to ask each participant the exact same questions but with the intention that the interview should flow with the conversation and questions to be added or omitted where appropriate. Where participants were unsure on a question or asked to clarify what was being asked, the 
thematic questions or prompts so to speak were referred to, to stimulate further conversation. For more information regarding the interview themes and question development, refer to Appendix 4.10 and Appendix 4.11.

\subsection{3: Interviews: Role of the Researcher}

Interviews are useful to explore the motivations for certain actions and decisions, particularly with topics in interpretive research such as CSR that have moral and ethical underpinnings. However, in order to nurture conversational style interviews and elicit responses in the participants own language (Spence \& Rutherfoord, 2001), it was important that throughout this data collection the researcher carefully selected open questions and remained flexible during the conversational turns of the participants (Liedtka, 1992).

As Liedtka (1992) explains, "rigor in interview methods is a state of mind on the part of the researcher rather than a property of his or her instruments" (p. 176). This suggests that there is a need for researchers to have "an attitude of skepticism, an attention to outliers, rival explanations and negative evidence" (p. 176) and feedback from informants and other facilitators of the research. Throughout the study this was taken on board and the researcher attempted to sustain flexibility and open conversation during the interview, tried to be consciously aware to not disregard any anomalies or divergent findings and finally to seek advice and feedback from the other facilitators and interview participants.

\section{9: Data Analysis}

\subsection{1: Content Analysis}

With a vast amount of qualitative data derived from the focus groups and interviews, there was a need to make sense of a large quantity of complex data. As Miles and Huberman (1994) explain, "data reduction is a form of analysis that sharpens, sorts, focuses, discards, and organizes data in such a way that "final" conclusions can be drawn and verified" (p. 11). One way of conducting this data reduction is via 
content analysis. There are a wide range of definitions for content analysis, but it can be described minimally as "any methodological measurement applied to text (or other symbolic materials) for social science purposes" (Shapiro \& Markoff, 1997, p. 14) whereby the aim is to "inspect all empirical data for recurrent instances, such as words, themes or discourses" (Eriksson \& Kovalainen, 2008, p. 187).

There are a number of key principles that are central to content analysis. One significant principle for this research is the "importance of language in human cognition" (Duriau, Reger \& Pfarrer, 2007, p. 6). The analysis of textual data such as transcribed voice recordings enables the researcher to understand cognitive schemas of participants (Gephart, 1993), with the occurrence and frequency of words being an indicator of cognitive centrality (Huff, 1990) or importance (Abrahamson \& Hambrick, 1997). Furthermore, content analysis acknowledges that underlying themes and concepts can be revealed by the reoccurrence of grouped language (Huff, 1990; Weber, 1990).

Content analysis is particularly appropriate for research of this nature because deeper moral philosophy and ethics underpin the notion of CSR. As Duriau et al. (2007) point out; content analysis is a useful methodology to "access deep individual or collective structures such as values, intentions, attitudes, and cognitions" (p. 6). SMEs are characterised by the owner-manager's role as central decision maker, with their personal values and business intentions being salient factors that influence CSR engagement. The collective attitudes and the way that SMEs make sense of and interact with CSR are also of key interest. Content analysis therefore aligns well for application to the management setting (Gephart, 1991; Ullmann, 1985), specifically to provide rigorous exploration of topics such as CSR that can be difficult to study using traditional quantitative methods (Duriau et al., 2007).

\subsection{2: Data Analysis Approach}

The analysis adopted for the focus groups was inductive and emergent rather than planned with pre-conceived categories. This means that the researcher immerses themselves in the data and instead allowed categories to emerge (Hsieh \& Shannon, 2005), "as the interactive passes of analysis and coding progressed" (Baden et al., 
2011, p. 267). This approach was valuable for the exploratory phase and acknowledges the context of the research situation. This scheme requires the grouping of similar responses, views or actions received from the participants to develop categories, subcategories, themes and concepts.

Alternatively, a comparatively a priori approach was adopted for the interviews, drawing from existing concepts from the literature to anticipate potential categories (Miles \& Huberman, 1994). These were guided by both the focus groups findings and the research focus on Spence's two redrawn CSR theories relating to stakeholder theory and the SBSR pyramids. Some examples include codes such as terminology, conceptualisation and motivations (micro - individual) and friendship, community embeddedness, reputation and collaboration (meso - organisational). However, this is not to say that the anticipated codes were fixed or restrictive, but to provide foundations to steer the analysis in valuable and novel directions. This allowed the potential to refine them as the analysis proceeded, with themes, patterns and subcategories again emerging inductively.

The combination of both a priori and inductive analysis reinstates the iterative nature of qualitative research and follows a parallel process and rationale to thematic analysis (Corbin \& Strauss, 2008), despite differing terminologies.

\subsection{3: Data Analysis Process}

The data analysis and coding procedure for both the focus groups and interviews was undertaken through a number of iterative steps, as displayed in Figure 4.6 below. 
Figure 4.6: Data Analysis Process Steps

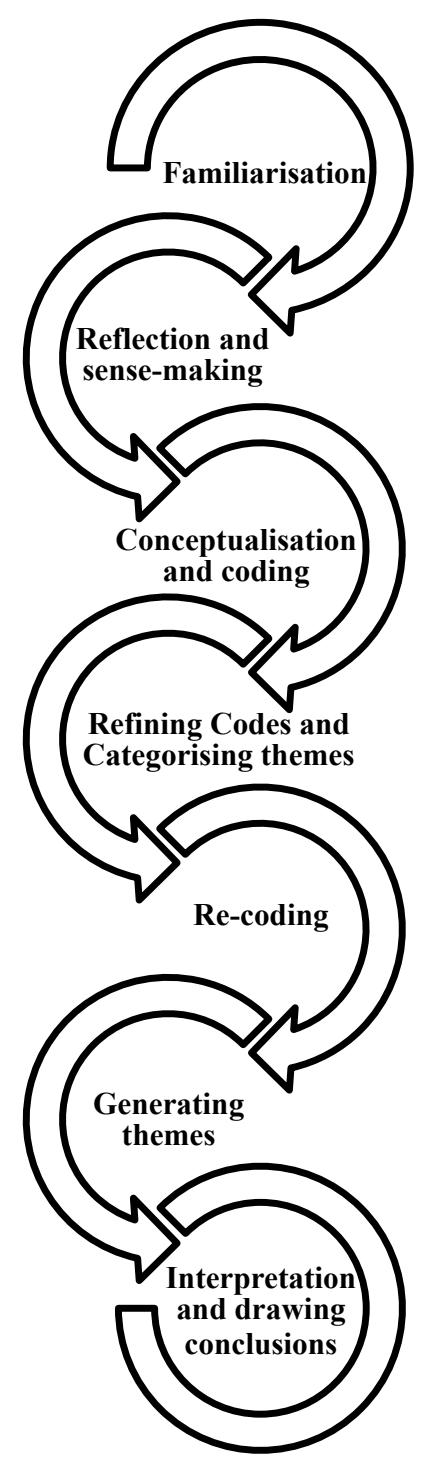

Step 1: Familiarisation (Easterby-Smith, Thorpe \& Jackson, 2012) - In order to analyse the findings of the focus groups and interviews, both were voice recorded and fully transcribed. This enabled fluidity with an unobstructed narrative and more accurate account of conversations (Bryman \& Bell, 2011), as proceedings were not stopped to accommodate excessive note taking. The first step of data analysis therefore required the researcher to listen to the voice recordings, transfer them into word documents via transcription, and read the transcripts multiple times. This enabled the transcriptions to be coded so that the thematic content analysis could take place. 
Step 2: Reflection and sense-making - The following step involved the researcher starting to make sense of the data by evaluating in it relation to previous literature on CSR in SMEs. In doing this, it allowed a critical comparison, establishing if the data supported or challenged our existing understanding. It also highlighted any novel ways that it diverged from these or identified answers for gaps in knowledge.

\section{Step 3: Conceptualisation and coding (Easterby-Smith et al., 2012; Miles \&}

Huberman, 1994) - Qualitative content analysis often adopts coding to bring some form of categorisation to large sets of data (Bryman \& Bell, 2011). This stage required the coding of transcripts by labeling words, lines or sections of text in order to make sense of them (Patton, 2002). By clustering the data (Miles \& Huberman, 1994) it allows the establishment of categories and sub-categories.

This first type of coding could be described as open or initial coding (Strauss \& Corbin, 1998), whereby the transcripts were analysed by line and assigned codes when felt appropriate. It is an unrestricted initial coding, with the aim to produce provisional descriptions of concepts that fit with the data (Strauss, 1987). This opens up the enquiry inductively and allows meaning to be assigned to segments of text, with provisional codes refined to the most appropriate once adequate coding had been completed. The closeness of the codes to the raw data increases the reliability of the analysis (Thomas, 2006), it also allowed for the assigning of new codes if the guidance codes for the interviews did not encompass the data. The open coding process was a starting point for further exploration of the concepts and themes that emerged (Saldaña, 2009), providing the foundations for the research questions.

Step 4: Refining codes and categorizing of themes - The open coding stage allowed the development of a set of core themes (Miles \& Huberman, 1994), capturing the richness of the qualitative data. Proceeding this initial coding, the second process then involved moving to higher levels of abstraction by distinguishing between basic level codes that link to higher-level codes (Corbin \& Strauss, 2008). This hierarchical structure could be described as tree coding (Bryman \& Bell, 2011) or pattern coding (Miles \& Huberman, 1994) whereby there is an arrangement of 
analytical sub-themes subordinating the higher-level themes (see Figure 4.7). This assists the development of the data and facilitates interpretation.

Figure 4.7: Coding Levels

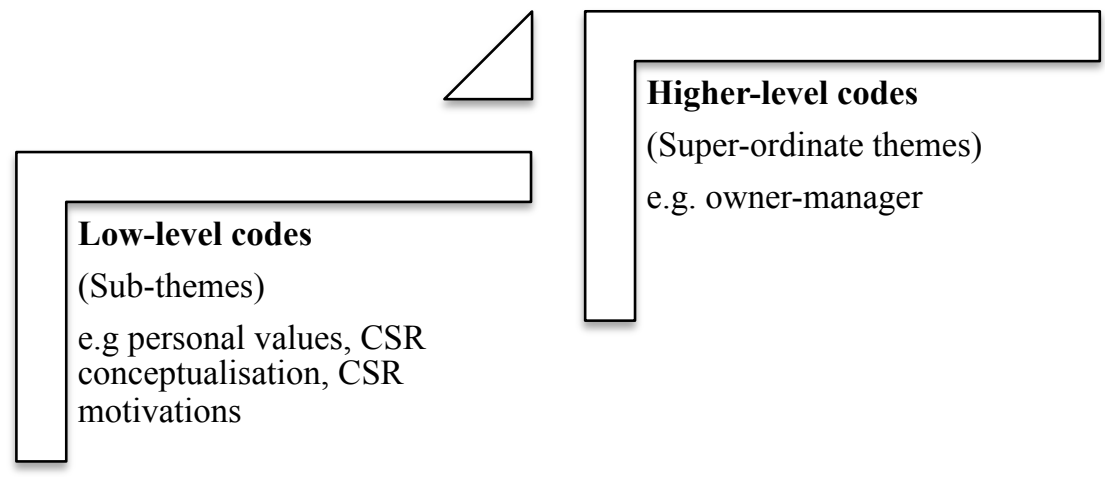

An example of these levels from the interview data would be personal values, conceptualisation and motivations of CSR being explanatory factors for the role of the owner-manager in CSR engagement. Again, as analysis advanced more prominent themes were moved to higher levels. Examples of the raw data analysis and coding process are provided in Appendix 4.12, Appendix 4.13 and Appendix 4.14, with a coding map in Appendix 4.15 as an example of data visualisation.

Step 5: Re-coding - The coding process is iterative (Baden et al., 2011) therefore there was a progressive refinement of codes and iterations of categories, although as Saldaña (2009) explains, this process could be described as more cyclical than linear with continuous comparisons between the data and codes (Corbin \& Strauss, 2008). This continuous comparison is similar to Goffman's (1974) work on 'frames', as Spence and Rutherfoord (2001) explain, when analysing data, iterative re-reading of the interview transcripts is useful to derive themes. It also allows consistency and a constant comparison and re-analysis of codes throughout.

Step 6: Identifying themes and linking data - This penultimate stage involved identifying linkages and patterns evident between the categories and key themes of the data. Chapter Five outlines the focus group findings and Chapters Six and Seven the interview findings with descriptions of the key themes. 
Step 7: Interpretation and drawing conclusions - Due to the thematic coding process, the researcher was required to be more involved in the process with a certain amount of interpretation because "the analyst is searching not just for manifest but latent content as well" (Bryman \& Bell, 2011, p. 298). Therefore, this stage required the researcher to build a conceptual framework by exploring and transcending the data to uncover deeper meanings and notions that underpin CSR in SMEs.

\subsection{4: Memoing}

Memoing is another important role of data analysis and for qualitative research in general. It is the process whereby the "researcher steps back from the details of the analysis to collect his or her conceptual musings, without self-censoring, on a topic of particular interest" (Liedtka, 1992, p. 176). Reporting thoughts, concerns and findings helps clarify to the researcher what they know, after all "writing is thinking, not the reporting of thought" (Miles \& Huberman, 1994, p. 101). This writing up of ideas facilitates the beginning of the analysis along with the coding and can uncover many layers of thought throughout the process, often helping the researcher to move from the descriptive, creative and speculative part of the analysis, to substantive or theoretical memos that suggest deeper, conceptual content. This was adopted throughout the data analysis of this research (see Figure 4.8). 


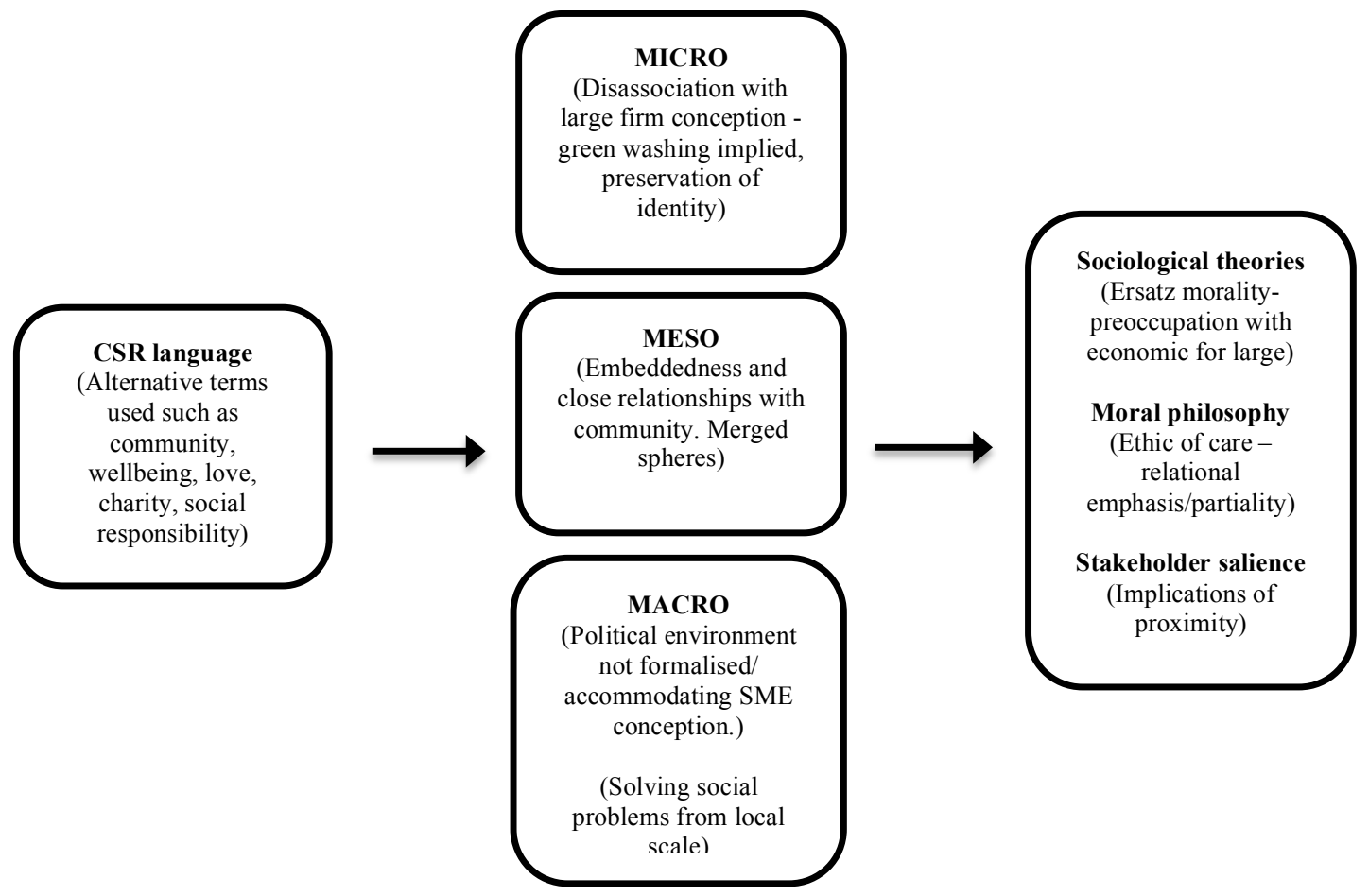

\subsection{0: Socially Desired Response Bias}

The role of the researcher in guiding both the focus group and interview proceedings has been discussed; this brings the topic of 'socially desired responses' to the forefront. The socially desired response bias regards the notion that participants respond in a manner that they consider is socially desirable. This could be argued as a particular consideration for research conducted in fields relating to questions of ethics and morality. However, this is not necessarily a truthful representation of their actual values, thoughts or beliefs. In fact, it is what Liedtka (1992) would call 'deliberate dishonesty'. Participants instead respond in a way that they think is morally or ethically acceptable, or what they perceive is meeting the expectations of the researcher (Blackburn \& Stokes, 2000) or society in general. This remains one of the most critical issues of validity for self-reporting methods (Liedtka, 1992).

It is difficult to avoid or in fact reveal such biases, however to enhance the validity of the interviews certain measures can be taken. One example would be ensuring that questions are in no way leading to an assumed response (Campin et al., 2013) 
and to acknowledge the biases of the interviewer previous to conducting the interviews to prevent 'contamination' of the research (Liedtka, 1992). Following this, during the interview it is the interviewer's responsibility to probe detailed conversations in order to gain the most accurate insight and reveal any inconsistencies in responses from participants. This is also the case during analysis where it is the researcher's job to interrogate the interview transcripts closely in order to establish the most accurate representation of the opinions of the respondents.

Finally, one further way of negating the possibility of socially desirable responses is to ensure there is minimal power distance (Hofstede, 1984) between interviewer and participant. Unavoidably the one-to-one interview can be seen as an "artificial setting" (Blackburn \& Stokes, 2000, p. 44), but attempting to reduce the power distance may dilute this issue. The focus groups also acted a way of reducing both the culture gap and power gap between the researcher and SME owner-managers, therefore the themes that informed the interviews are less likely to be tarnished by the social desirability bias.

\subsection{1: Validity}

There has been unease with evaluating qualitative research using credibility and reliability (Guba \& Lincoln, 1994). This unease is largely due to the assumption that there is one single and absolute account of a social reality. However, a contrasting view is that there is not necessarily one absolute truth, but multiple accounts of reality. With regards to this assumption, the quality of the data relies on interpretation. To ensure that the interpretations of the researcher did not intrude into the richness of the data, attempts were made to keep them as close to the data as possible. Precautions were taken so that the researcher did not over-interpret; one of these being the substantial amount of raw data interposed throughout the findings chapters. This enabled the reader flexibility to independently make sense of the interpretations.

There were a number of ways that the validity of this research was further taken into consideration, for example, through the collection of multiple sources of evidence 
from a mixture of research methods. These were inclusive of focus groups and interviews, with participant observations made throughout data collection via both the interviewer and additional facilitators during the focus groups. The use of field notes and voice recordings for each data collection method was also important to 'safeguard' both validity and reliability and is generally just good practice (McCracken, 1988; Liedtka, 1992).

More general documentations were also kept of each stage of the research process. This included how the research instruments were developed, how and why certain participants were chosen, how the themes and questions for both the focus groups and interviews were established and how the data analysis was undertaken. This enabled a story to be established and clear evidence of how the research developed in order to justify why each step was taken.

Finally, internal validity or what Guba and Lincoln (1994) term credibility assesses whether there is coherence with researchers' observations and the theoretical ideas that are developed. As Bryman and Bell (2011) explain, it can often be a strength of qualitative research because of the prolonged participation with a certain group of participants. This allows a higher level of congruence between concepts and observations (LeCompte \& Goetz, 1982). However, respondent validation is also a measure that can be taken to ensure credibility and internal validity of the research.

\subsection{2: Reliability}

Reliability or dependability (Guba \& Lincoln, 1994) was ensured via a number of different avenues. For example, establishing a sense of auditing whereby an accessible record was kept of each phase of the research process (Bryman \& Bell, 2011), as already discussed above. This included a clear story from the research questions, literature review and focus groups that led to the development of themes to inform the interview questions. The owner-managers were selected from businesses that strictly adhered to the European SME definition. For the focus groups specifically, participants were selected from a trustworthy database of SMEs from the FSB, with internal reliability ensured by having more than one facilitator in 
each focus group. This enabled accounts of the proceedings to be compared during discussions following the focus groups (Bryman \& Bell, 2011).

Despite the questions being flexible, the themes of the interview instrument that were derived from the focus groups were followed consistently throughout the research to enable comparison. Furthermore, the analysis process followed the lines of previous research (Ibrahim et al., 2012; Lädesmäki \& Takala, 2012) and coding procedures advocated in the literature (Corbin \& Strauss, 2008; Easterby-Smith et al., 2012; Miles \& Huberman, 1994).

\subsection{3: Limitations and Boundaries of the Research}

Attempts have been made to try and eliminate limitations as much as possible throughout the research via the rigor of the research design developed. However, Krippendorff (1980) believes, as cited in Liedtka (1992), that the nature of data and the fact it originates from "complex symbolic forms in an indigenous language" makes it difficult to analyse in its "original manifestation" (p. 170). Furthermore, it is interesting to note that the unique advantages of this research perspective and methodological approach can also be considered as the main limitation, this being the interpretivist nature. Interpretivist research relies on the interpretive skills of the researcher in conducting and analysing the research. They are an active and intrinsic part of the research. However, the limitations of the role of the researcher have been acknowledged previously in this chapter and attempts have been made to overcome these by ensuring validity, credibility, reliability and dependability.

This research is further limited to what the owner-managers were willing to disclose of their opinions, engagement and reasoning of the notion of CSR and the extent to which the conveyed information was accurate (Campin et al, 2013). Respondents further have a limited capacity for recall and introspection on events (Liedtka, 1992). Measures were taken to eliminate such issues as explained in the above paragraphs, yet it is important to acknowledge these challenges when analysing and discussing the findings on the phenomenon of CSR in SMEs. This acknowledgement will help to relay a more representative understanding of the topic at hand. 
Finally, external validity or transferability can often be seen as a limitation because it can be difficult to generalise qualitative research across settings. This is due to the reasonably small sample sizes of intensive study in unique contexts. Instead, Guba and Lincoln (1994) suggest that emphasis should be put on the breadth of the research. This entails thick descriptions (Geertz, 1973) and rich accounts of the social world being studied. Such thick description provides a basis with which others can then make judgments as to the possible transferability to other settings.

With all of the above challenges taken into consideration, these limitations are outweighed by the advantages of an in-depth qualitative approach that was necessary to answer the research questions. The original research design for this study was mixed methods in order to use the Implicit Attitude Test to reduce the social desirability bias or to test the appropriateness of the four redrawn domains for CSR in SMEs (Spence, 2016) with Carroll's (1979) original quantitative assessment instrument. The issue that arose was that during the focus groups, SME ownermanagers had a lack of understanding and divergent interpretations of what CSR meant in an SME setting. The use of quantitative research or in fact mixed methods would risk oversimplifying this extremely complex and context dependent topic and therefore it was not warranted. Consequently, the opportunity was taken to develop a qualitative approach utilising in-depth exploration and a contextual understanding of the complex phenomenon of CSR. It is suggested that fresh insights can be found by utilizing this truly qualitative stance to research (Cornelissen, 2017).

Cornelissen (2017) further explains, "the hallmark of qualitative research in producing rich and detailed explanatory accounts of management and organisational phenomena is under pressure" (p. 369). As he contends, perhaps it should not be favoured or stubbornly argued for one particular method or style of theorising, but appreciation of the strengths and weaknesses and the ability to balance such research styles for more refined insights into the phenomena under study (Abbott, 2004; Cornelissen, 2017). There are many different ways of explaining phenomena, so as long as the methods chosen fit with the story and allow the researcher to say everything that they want to say with methodological rigor, then this will enhance and cultivate more complex understanding and enriching contributions. This is 
particularly so when such ways of explaining are considered alongside each other and not as competing.

\subsection{4: Ethical Considerations}

This research was not considered to pose any ethical concerns to the wider society; the main issue was that of anonymity and confidentiality with regards to participants of both the focus groups and the interviews. A further concern inherent in qualitative studies is ensuring no harm comes to the participant through the subsequent use of their testimony (Rubin \& Rubin, 2004). Considerations were therefore made regarding informed consent, confidentiality, anonymity and honesty regarding the future use of data.

The first stage of the process was to gain a light touch ethical approval from the University ethics board. This was done prior to any research being carried out to ensure that the study posed no risk to potential participants. When approached, participants were informed of the qualitative nature of the focus groups and interviews and it was explained that their contribution would be used as part of a $\mathrm{PhD}$ thesis. In the focus groups participants were told that the topic was CSR and for the interviews that the focus was on the way that SME owner-managers prioritise their responsibilities to the economy and society. There are tensions between informed consent and the avoidance of setting a priori boundaries in what is an interpretive study, but it was felt that this information provided a position adequate of informed consent.

Following this, at the start of each focus group and interview a verbal conversation was carried out explaining the proceedings and assuring confidentiality. Each participant was required to sign a consent form that was situated within the participant booklet; this confirmed their consent or objection for the focus groups or interviews to be voice recorded. All participants agreed to be voice recorded. On two occasions an interview participant asked for the voice recording to be paused momentarily for a sensitive topic; however recording commenced soon after this information had been communicated. Furthermore, the labelling of participants 
ensured anonymity, identifying individuals from P1 upwards for both methods of data collection and the location of the focus groups were altered to Location 1-4. Any other clues, names or references that might reveal the identity of the individuals or their businesses were altered. This removed the identity of the individuals and ensures confidentiality and anonymity.

\subsection{5: Chapter Conclusion}

This chapter has outlined the ontological and epistemological position taken during the research, defined the research strategy and justified the methods and analysis used. The interpretive and social constructionist perspective was considered to be congruent with the research questions and appropriate to derive the level of detail needed to explore CSR in SMEs. To ensure methodological rigor, issues of validity and reliability have been covered in detail and the ethical considerations of the research outlined. The following chapter of this thesis will now present and analyse the focus group findings and outline key themes that informed the in-depth interviews. 


\section{Chapter Five: Focus Group Findings - The Social Construction of CSR in SMEs}

\section{1: Introduction}

This chapter presents the findings of the initial exploratory phase of data collection, conducted via focus groups. The full rationale for this method is in Chapter Four. However, to recap, focus groups were utilised to provide a valuable insight into the real-world views of practitioners on the topic of CSR. This initial phase of data collection and analysis helped inform and refine themes for the in-depth interviews that followed. SMEs are still an under-investigated context for CSR; therefore an exploratory analysis was useful to validate the interviews as an appropriate methodological approach for the main phase of data collection.

The focus groups were conducted between May 2016 and August 2016, before the subsequent data collection was carried out from November 2016 onwards. It is of value to acknowledge time scales as the societal perceptions of social responsibility are under continual construction. As discussed in Chapter Four, this stage of research consisted of four focus groups spread throughout Wales, integrated into the regional FSB meetings. Participants included owner-managers and key decision makers of SMEs within the FSB membership.

The rest of this chapter presents the focus group findings and is organised into eleven super-ordinate themes (see Appendix 5.1 and Table 5.1) that emerged from discussions. These are supported with examples of rich dialogue and extended explanations. The sub-themes (see Appendix 5.1) are not made explicit, but guide the content within each super-ordinate theme. Following this, the findings are summarised and the influence on the subsequent stage of data collection is outlined. 
Table 5.1: Focus Groups: Summary of Super-Ordinate Themes

\begin{tabular}{|c|}
\hline Super-Ordinate Themes \\
\hline CSR Terminology \\
\hline Social Identification \\
\hline Owner-Manager Values \\
\hline Drivers and Motivations \\
\hline Community embeddedness \\
\hline Stakeholder Relationships \\
\hline Stakeholder Prioritisation \\
\hline Barriers and Challenges \\
\hline Economic, Political and Social Influences \\
\hline CSR Awareness \\
\hline CSR Impact \\
\hline
\end{tabular}

\section{2: Terminology Matters: The Rejection of CSR}

The first theme regards CSR terminology and the language used by participants. Language can uncover truths and locate the context in which the concept of CSR is socially constructed. As discussed in the literature review, there is discursive ambiguity with regards to the CSR term. This was noticeable in the findings of the focus group with twenty-one different terms being used. There was also a disassociation with the language of 'corporate social responsibility', with ownermanagers not identifying with the word 'corporate'. This is seen in the extract below:

P24: "You're right, the word corporate is the problem because everyone's thinking, 'oh that's not me then'”. (p. 17, 487-488)

As P18 explains below, SMEs do not identify themselves as corporate businesses. The term CSR was therefore considered as excluding and did not sit comfortably within the frames of their known realities: 
P18: "I don't like that, the word corporate it is...excluding, it excludes, just a few moments ago I just said $95 \%$ of businesses in the whole of Wales are SMEs, they really do not consider themselves corporate at all". (p. 21, 625-627)

Owner-managers in the focus groups were commonly describing engagement with initiatives that are considered CSR, yet not naming them. Such silent CSR is a common characteristic of SMEs and is evident below, whereby such activities are simply seen as the 'right thing to do' and integrated as part of everyday business operations:

P6: "A lot of people don't really think they do half of what we discussed, probably because they don't call it anything, it's just a case of we do the right thing and that is it". (p. 20, 593-595)

Language when used by SME owner-managers ranged on a conceptual continuum from individual morals and emotions, organisational community level understanding, to the universal principle of humanity. This spectrum of language attached to the notions encapsulating CSR is evident in the extracts below:

P5: "We just call it love". (p. 17, 507)

P17: "Community is what I think, that's on the records you know, I've always thought community was social responsibility". (p. 21, 633-634)

P18: "Why don't we just be inclusive of the whole world's situation and just call it humanity isn't it, you know because this is about our individual and collective humanity". (p. 21, 639-640)

What is clear in these findings is the discursive ambiguity and disassociation with the term CSR. That being said, the concept and notion is very much alive in SMEs. When called anything at all, it was most commonly socially constructed with reference to morals and ethics, or derivatives of community engagement. This lack of a common terminology for CSR in SMEs can be limiting to owner-manager's understanding of the concept. A drive towards solidarity of the language could consequently facilitate awareness and understanding of the concept. 


\title{
5.3: Social Identification: "More Socially Responsible as People"
}

The social identity of owner-managers refers to their individual self-concept based on group membership. Owner-managers seemed to engage with CSR to express their social identification, for example, a sense of belonging to the community or pride of being an SME. However, it became apparent that SMEs also disengage with the term 'CSR' as a technique to preserve their social identity. There was both unintentional and intentional disengagement with the term. The first due to a knowledge gap and lack of understanding, and the latter refers to a conscious desire to disassociate themselves and their SME from the larger corporate conception of the term. This is due to the perception that there is a mismatch in large businesses, where they may have departments dedicated to CSR, but their business operations are not considered ethical. P27 explains this misalignment in the extract below:

\begin{abstract}
P27: 'I'd just rather have a term like ethical business, because to be honest I think that you can have big companies who have got a really strong corporate social responsibility team and department, but actually their business isn't actually very ethical at all, so there's a mismatch". (p. 19, 558-560)
\end{abstract}

Such intentional disassociation seen above is driven by the desire to preserve the social identity of the owner-manager and their SME. In fact, it was common in the findings that participants referred to their identity as an SME owner-manager and perceived themselves and their businesses as more intrinsically engaged and ethical due to this group association. In comparison, they believed that the CSR concept for large businesses is a balance sheet task, with profit being the ultimate driver. This is evidenced below by P6:

P6: "It is a grass roots thing around this table, but for bigger corporations, it is just a balance sheet thing and trying to you know, pass off that ultimately it comes down to profit, whereas as I was saying, from what we've heard here tonight, I think we've all got a genuine wish to and that is why we do it". (p. 18, 525-528)

Such a negative view of CSR in large corporates is again replicated by P19. They view the labelling of CSR as an accountability act to create tangible outcomes in the 
form of numbers. A valid point is made that despite corporates communicating large impact cases as an outcome of their CSR efforts, collectively SMEs also have a huge impact on societal wellbeing:

P19: "It has been labelled as corporate, because that is something tangible for people to see, so you know when you talk about corporate you see the big factories you know you see the output, you see the big numbers and you think more impact but actually, you know, if you've got 95\% of those businesses which are small you know versus those big businesses there's more impact, you know, from the smaller ones". (p. 22, 655-660)

Consequently, disassociation with the term CSR is seen to protect the social identity of SMEs and allow them to position their CSR efforts as driven by more genuine motives rather than bottom line implications. This identification of SMEs and individuals as more socially responsible is again notable in the two separate extracts below:

P25: “We're more able to do this stuff more readily because it's us [emphasis by participant], we are more socially responsible as people, I mean I've got this belief that small businesses are intrinsically [emphasis by participant] more socially engaged than large businesses and therefore have more value to the economies". (p. 22, 641-645)

P13: "Don't you think you have to be a certain type of person to run your own business anyway, so maybe inherently we've got nice characteristics?" (p. 18, 492-493)

P25 and P13 claim that SMEs are more intrinsically engaged than large businesses, with owner-managers possessing inherently nicer characteristics. It is implied that CSR is inextricably linked to the individuals within the business, with SMEs contributing more value towards overall economic prosperity. The association of individuals and their implicit orientations towards social responsibility was a dominant finding for the social construction of CSR from SMEs. Owner-managers commonly viewed the concept as inextricably linked to personal responsibilities, as seen below:

P20: "I think that the other thing as well, with corporate social responsibility, it implies responsibility for the corporation as a legal 
entity, but everyone needs a lot more...personal social responsibility as well”. (p. 21, 628-630)

These findings imply two key points. First, CSR for large corporates is perceived as a concept motivated by profit. Therefore, SMEs disassociate with the term and instead refer to derivatives of CSR with regards to personal responsibility, ethics or community. Their conceptualisation of CSR and perceived responsibilities are therefore unique to their large counterparts. Second is the intrinsic engagement of owner-managers and their embedded role in the inception and engagement with CSR.

\section{4: Owner-Manager Values: An "Embedded Principle"}

A key finding of the focus groups was the importance of social responsibility as a personal duty as well as organisational. This brings the values of the owner-manager into focus. It was identified in the literature review that they play a dominant role as centralised decision makers in SMEs. P18 explains the importance of those individuals, viewing social responsibility as an embedded principle and not just an add on. The below extract refers to this perception of CSR:

P18: "It should be part of what we do, in everything we do, not something that's just an add on, shouldn't it be the way that you do everything that you do...embedded". (p. 18, 551-553)

Findings suggest that owner-managers view their business as an extension of themselves. One owner-manager claims that being socially responsible is the starting point for their SME as it is built on the foundations of their own personal morals. This is seen in the conversation below:

P27: "For the small, especially micro businesses, that is our starting point, so that's where we are as people, so what we're not doing is doing something because the company says we have to, we're actually doing it because we know it's right".

P24: "Yes, we don't have to be taught, it is coming from personal values". 
P25: "I think that's probably the most important thing I've heard yet, that point about small businesses or micro businesses particularly, are more able to be responsive to this stuff because we're doing it as socially responsible people, we're not dealing with the bureaucracy of a large organisation". (p. 9, 248-257)

This embeddedness of values throughout the business means that CSR efforts often reflect the individual's morals and the way they make sense of their responsibilities. This is perceived as a unique benefit to SMEs, giving them the ability to be responsive to societal or community needs. The importance of being aware of community needs on a personal level was considered by P14. They specifically feared that growth of their SME could result in the diminishing of links and the personal touch that keeps the business in sync with the local community and their needs:

P14: "Certain large local companies, they get so big that they bring in people outside of the community and then those links are lost. Again, we're going back to, you know, our own businesses, that's the one thing that scares me, if we grow and grow I don't want to lose that personal touch whereby we're in sync with the community and the needs of the community. I just think that's a key link, but the larger businesses, unless there is any kick back for them they're not really you know, their ethos is different, they're not in it, their personal values are not translated through the company. Whereas ours, because it's a smaller company, our own individual values can translate through the company and into the community easier". (p. 20, 539-551)

In terms of CSR engagement, one example of this personal element of CSR was the preference of the donation of time and skills, as is articulated below:

P25: "It seems to me, one of the biggest contributions that I make as a business is giving my time for free, for public interest things". (p. 9, 260-262)

Often SMEs are limited in terms of resources or capital so this is an accessible form of CSR. It also requires more of a personal investment by the owner-manager, giving their time and skills to assist. As seen above, CSR of this nature is implied to be mutually beneficial in terms of developing relations with key stakeholders; this will be discussed in later themes. A further finding is the preference of direct 
sponsorship and charitable efforts towards those with personal significance or causes in their local communities. The inclination to give time to community projects is seen below:

P14: "The up front, the invisible things are giving donations to local clubs, sports clubs, schools, school clubs, school fete days...the local college that kind of thing, sometimes there is a bit of kick back advertising, but I suppose the biggest thing for me, which is arguably more valuable really, is giving my time to...certain community projects". (p. 5, 126-130)

Multiple owner-managers displayed partiality towards charities that have a personal significance. It was considered valuable that they could see what the money was going towards, as a way of legitimising their charitable efforts. Two examples of this are shown underneath:

P20: "We choose to support Maggie's because it's something that we have a personal connection with, you know we can, we can physically go there and see the facilities that our money is providing”. (p. 10, 307-309)

P7: "We like to choose our own charities, we have four a year where we feel there is a level of personal connection". (p. 13, 365-367)

P7: "It's not personal when you say can I have some money". (p. 13, 370)

There was a cynicism towards the distribution of money in large charities, again strengthening the preference to direct philanthropic efforts towards community based, personal charities in order to help the community that the owner-manager is physically located:

P17: "What I found when I set up a charity is that all the small charities were doing good work, but all the big boys were eating it up and it wasn't going back to where you live". (p. 10, 310-312)

P17 further exclaimed:

P17: "I was really shocked to see how much money goes to the top tier and how little is left for the people”. (p. 9, 269-270) 
Finally, owner-managers consistently socially constructed CSR as an individual responsibility, to the point where it was considered a personal duty to ensure CSR efforts were directed to the right people and actually assisted the recipients in some way:

P17: "CSR you have to be personally responsible for. When you're giving it actually where is it going? Who is it actually helping? Is it helping the people who I feel that I want to help with my business?" (p. 10, 302-303)

One owner-manager considers that individuals have power in their pockets and the way that money is spent is a responsibility. They refer to a misalignment with actions and motivations, suggesting that people are often doing the 'right thing' but with the wrong motivations:

P18: "One of the things that I've always considered is the power in your pocket. We all go around with our loose change and we spend money, how we spend it, is actually a responsibility". (p. 12, 361364)

P18: "You have power in your pockets to make good and to influence and change people's attitudes to what they're doing." (p. 12, 370372)

P18: "There's a lot of people that actually do the right thing, but not necessarily for the right motivation and therefore they're actually undermining the achievement by not having the right motivation to back it up". (p. 12, 374-376)

The perception that there is a need to be responsible with CSR seems peculiar considering that the concept is being socially responsible. The misalignment between motivations and engagement in large businesses is implied as the reason why ownermanagers disassociate themselves with the term CSR as a large business activity. As a large business activity, it is seen to be driven by profit motives, rather than being a morally grounded activity or to assist the community. If SMEs are seen to be supporting the large business conception of CSR, then the personal reputation of the owner-manager may be compromised. Findings suggest that there needs to be a remoralisation of the CSR concept in larger corporates in order for SMEs to engage with the terminology. 
This theme emphasises the need for research that takes into account the role of owner-managers in the inception and engagement of CSR initiatives. Findings suggest that implicit orientations and personal interests towards societal responsibilities often drive CSR efforts. Therefore, in order to understand and explain the way that CSR is socially constructed in SMEs, context specific research is required to reflect their heterogeneous nature and the differing personalities and values of owner-managers.

\section{5: Drivers and Motivations: "The Right Thing"}

The principal motivations for CSR in SMEs originated from a perceived moral obligation to give back and the need for relational development to aid survival. Owner-managers seemed to be intrinsically motivated to do what was perceived as 'the right thing' and portrayed a sense of duty to assist their local communities or those stakeholders that may not have adequate resources or skills. Both of these collectively contribute to the betterment of society. For example, drivers for CSR initiatives recalled by participants commonly depicted the notion of giving back, as is evident in the extract below:

P1: "It's giving something back isn't it [sounds of agreement]". (p. $17,493)$

Furthermore, in response to CSR being driven by 'love', as was discussed earlier in section 5.2, P7 describes a sense of duty as a more accurate descriptor for her motivations:

P7: "I couldn't possibly use the word love I'm afraid in my context [laughter] I think all my clients would think I'd gone soft, I think there is a duty, I certainly feel a duty to something”. (p. 20, 578-581)

Empathy towards smaller communities with a lack of funding and resources was also seen as a driver for CSR. P14 described this sense of duty towards close communities as a trigger for his CSR engagement, as the following extract illustrates: 
P14: "The local football club wanted a new pitch, but they didn't have the skills, the expertise, or the money to go out to a consultant to say can you budget this for us and plan it, so I donated my time to do that". (p. 5, 132-134)

F1: "And did they approach you, did you already have an interest in the club?" (p. 6, 135)

P14: "I did have an interest in the club but yeah they approached me and you sort of feel a need to do it, because like most clubs in, especially rural areas other than anywhere, there's a lack of funding so they will rely on charitable gifts, donations, or in this case time, for people to give their time and expertise”. (p. 6, 136-140)

These motives are influenced by individual moral obligations to give back and provide time and resource to recipients that are in need. Further drivers for being socially responsible were relational. One owner-manager attributed their drivers of CSR to the promotion, reputation and legitimacy in their community via informal communication channels such as word of mouth:

P10: "I'm very aware of what we are trying to do here, if I am not working with the local school, the local head teacher, the local carers group, Age Cymru, any of those potential beneficiaries then...well round here word of mouth is the best kind of recommendation for what we're doing". (p. 11, 303-307)

Whilst this was a key driver for P10, many acknowledged the relational benefits, despite it not being the initial driver. P14 claims that egotistic drivers motivate many businesses, yet SMEs in this case acknowledged indirect benefits, but strongly deny that those are the key drivers for participation:

P14: "I do think there are businesses that just do it for 'oh look my name is on this, my name is on that' and it's an ego thing and it's certainly not that with me". (p. 12, 321-322)

P16: "I certainly don't do it for that particular reason, but there are obviously indirect benefits when people will go 'oh he did that there he did a good job he could do it for us'”. (p. 12, 323-325)

Consequently, the key drivers recalled by participants in the quotes are rooted in morals and giving back, with the acceptance that CSR aids in the establishment of relational ties in order to assist communities and society at large. Closeness to 
causes or human actors both physically and emotionally appear to heighten the moral impulse of owner-managers to behave responsibly.

\section{6: Community Embeddedness: The "Double Edged Sword"}

SME owner-managers often adopted community interaction synonymously with the concept of CSR. SMEs are characterised as closely embedded in communities with a reliance on webs of relations with key stakeholders. This was evident in the findings, with participants displaying a sense of responsibility to enhance the community that they are embedded within, as seen below:

P5: "The business has a duty as a guest to that community and a responsibility to enhance that community and make it a better space and a more positive space for them to live, a healthier and happier place to live". (p. 3, 65-67)

Many CSR efforts were directed towards this sense of duty to enhance and service their community. Most owner-managers claimed to prioritise a local supply chain as a form of social responsibility; this was driven in one case by a sense of belonging and an appreciation towards the locality for facilitating their lifestyle:

P18: "I try to use local for everything, I don't buy anything outside unless I have to because I believe that people in this area give me my lifestyle and I should support them and my local supply chain”. (p. 2, 45-47)

This participant continues to describe the need for good relationships with key stakeholders, describing them as reciprocal and mutually beneficial for both actors. Respect was considered a key attribute to sustain these reciprocal relationships:

P18: "I get a lot back from my local supply chain by being respectful of them and working with them. They don't work for me and I don't work for them, it's a relationship". (p. 2, 52-54)

The upkeep of reputation within the community was seen as vital to sustain business operations. One owner-manager of a professional service SME emphasises this point: 
P7: "Reputation is pretty much everything, you can build a business on your contacts here". (p. 5, 143-144)

P7 refers to reputation and personal contacts as a means of growth for SMEs. The use of informal networks to build business is further elaborated with discussion on word of mouth. As P11 explained, all of their work was repeat or via word of mouth:

P11: "Word of mouth, I was actually saying it's so important because I've only had a website for a year, because everything was repeat work and word of mouth". (p. 12, 327-328)

SMEs reinforce reputation within communities and legitimise business operations via their CSR efforts and community involvement. Although, P7 point out that such closeness to the community can be a double edged sword:

P7: "You get found out in a community if you're not very good, word of mouth, I know the bad plumbers as well as the good plumbers, it's a double-edged sword". (p. 5, 136-138)

Community embeddedness can consequently be seen to increase pressure for SMEs to maintain their social identity and behave responsibly according to stakeholder expectations. P7 further contests that community relations do not substitute the need to provide a good service. It is suggested that once competency based reputation has been secured, enhanced links with the community will be a consequence of the trust gained:

P7: "I do appreciate the need for links with the community, but I think certainly in our commercial sort of practice, you've got to be good at your job. If you're good at your job you'll always do well and do even better with links with the community". (p. 5, 131-134)

Owner-managers commonly referred to community relations being reciprocal, mutually beneficial or defined by the word collaboration. One interviewee described his community set up as unique whereby if supported, it pays dividends to the SME: 
P6: "I think that we do have a unique community set up, certainly down in the valley you know, providing you work within that community". (p. 5, 123-124)

P6: "You've got to get involved with the community and support it and be fair to it and it will pay you dividends [noises of agreement]". (p. 5, 127-128)

This notion of reciprocation is also encompassed by the word collaboration used by P10 below:

P10: "Is it social responsibility? No, it's just what decent people do in a way. We are a social business, our raison d'être is about mobilising, up skilling and engaging and collaborating with the local community and a key word for us is that collaboration, which implies this sense of reciprocity, but it's not a case of you know of 'if I do this for you will you do that for me', it's a much more kind of organic thing, that when the opportunity comes up, who could I ask, knowing that at some point, that emotional credit may be used the other way around". (p. 7, 184-191)

Community collaboration was seen as an organic process in which favours are reciprocated when the opportunity may arise. Interestingly, this was not seen as a social responsibility, but as a result of personal morals and an output of the SME as a 'social business', with 'emotional credit' being described as a driver for reciprocation. In this case social responsibility as a term was actively defied, despite the dedication to servicing the community and ultimately the upskilling and development of society.

A final summarising point made by P25 refers to the value of SMEs embeddedness within communities. It is suggested that strong connections and identification to a particular location is the recipe for a more sustainable economic, social and environmental model for SMEs. This point is made explicit in the extract below:

P25: "Small businesses are more integrated within their local communities than large businesses are. They are by their very nature, more connected to a particular location. That for me is the recipe for a more sustainable economic, social and environmental model and big businesses are far less likely to because they're dependent on international capital flows". (p. 41-42, 1228-1232) 
The participant follows up on the value of SMEs situated in communities. He refers to supporting organisations such as the FSB as being crucial to raise awareness of the value SMEs contribute to their localities. This is described below:

\begin{abstract}
P25: "We are by and large pretty rubbish at actually selling our own virtues, I think it takes organisations like the FSB, doing stuff like this with the manifesto, to do the kind of stuff that shows the value that small businesses actually give to our communities, but we don't do enough of it". (p. 42, 1234-1240)
\end{abstract}

It is obvious that there are both positive and negative implications of being physically proximate to key stakeholders such as the community. P25 considers it a necessity for more sustainable and responsible models for SMEs. However, the nature of the business environment is changing and the role of embeddedness and closeness to key stakeholders needs further consideration.

\title{
5.7: Stakeholder Relationships: "An Element of Mutual Care"
}

Community was not the only SME stakeholder whereby relationships were nurtured. Participants showed a sense of care towards diverse webs of key stakeholders, specific to each SME. Stakeholder relationships were more nuanced and complex than a simple set of dyadic relationships. This is particularly evident as the findings progress in this sub-section. As an initial finding, rather than viewing CSR engagement as a social responsibility, one owner-manage considered it as simply caring for people:

\begin{abstract}
P10: “There's almost an element of mutual care, you're talking about teaching I think it's more than that, I think it's actually genuinely caring for somebody, and if that gets them over a bump and they keep going, that's great for them, well I personally feel a sense that I've helped somebody at that point". (p. 18, 487-490)
\end{abstract}

A further example from the findings builds on this perspective, describing the nurturing of close relationships with key stakeholders as vital for the survival of the SMEs. These relationships are developed over time, as is seen below: 
P16: "The various subcontractors who organise shows ...they're vital as far as I'm concerned so I have a very very good working relationship with them. And that's built up over, over time". (p. 4, 81-84)

He explains that these relationships were established from personal contacts and previous employment over a number of years. The theme of personal contacts initiating business relationships is carried on in the quote below, being described as vital for the SME:

P16: "I've known them for a number of years and have worked for them previously so it's just carrying on that relationship... and it's vital, it really really is “. (p. 3, 77-79)

Additional to the close relationships established from personal connections and nurtured over time, findings suggested that webs of stakeholder relationships are built from the local community as the core catalyst. P4 refers to community as the base, with webs of relations organically branching out over time. He cites the use of local suppliers as a specific example of business responsibility, supporting prosperity in the local economy:

\footnotetext{
P4: "You also have to have that responsibility in a sense that you look within your immediate vicinity to what suppliers you have. How you can support them by using the businesses that you've got within your immediate local economy, to be able to support the economy? Then you have a sort of cloud base that you can work from... and then from that you can then promote that you're using the local suppliers and relationships grow from that and sort of branch out like a web from that point...I think it's incredibly important that you support your local community". (p. 3, 81-88)
}

Creating relationships and keeping business within the local economy was seen as a big responsibility for many of the owner-managers. This was enabled via the development of long-term relationships over a number of years, most often in the local vicinity:

P6: "I would say that it is absolutely essential, certainly in our business in hospitality that we have really good, strong links with stakeholders like local food suppliers, which we use on a regular basis obviously and have done for 13 odd years". (p. 4, 104-107) 
Strong relationships were often seen as mutually beneficial for both the SME and their stakeholders. P6 above explains that benefits of creating a core team of stakeholders often includes preferential treatment and quick service in return for regular business over the years. Relationships such as these service the local community and encourage sustainable prosperity in localised regions.

P6: "Because we've stuck with the same core team if you like over the last 13 odd years they get regular business and they get paid well and we really work well together, yeah we do jump the queue, we do get preferential treatment and it does pay”. (p. 4, 109-112)

It is important at this point to re-emphasise that relationships were not simply dyadic. Instead, they were complex, often involving numerous connections between multiple actors. The nature of these actors was dependent on the context of the SME. Collaborative relationships with multiple stakeholders were utilised by participants. On numerous occasions SMEs worked in collaborative teams, these teams had synergistic implications, enabling SMEs to engage in business activities not otherwise viable as single entities. For example, one owner-manager describes their approach towards business as being joined up and collaborative with stakeholders such as educational institutions:

\section{P18: "I work in collaborations with lots of others so I associate with people, they associate with me and I'll work with lots of Universities and Colleges. So the collaborators, so it's all about this kind of joined up approach and that then goes into local connections. That's how I see it”. (p. 3, 68-71)}

P25 touches on the same point below whereby collaboration mobilises projects that are bigger than their ability as a singular entity. These reciprocal relationships open up doors for SMEs to be part of larger projects, develop contacts and increase potential business opportunities. This contributes to the survival of the SME and provides potential avenues for growth. Relationships such as these were often enabled by close physical proximity to stakeholders, or social ties between two actors. 
P25: "I work with associates on other projects so sometimes I might do stuff that is bigger than my ability to do on my own".

F1: "Yeah".

P25: "So I will work with other researchers, other independent researchers, I don't employ anyone so where appropriate, will work with others".

F1: "What would you call that?"

P25: "Research collaboration, but you know, if we're putting bits together, if it's clearly beyond my ability to...to do work then I will work with others and likewise they will bring work to me". (p. 1-2, 22-30)

There are two key points that emerge from these findings. The first is that stakeholder relationships are bespoke, dependent on the context of the SME and personal contacts of the owner-manager. Webs of stakeholder relationships are often developed within communities as the central catalyst and initiated by these personal contacts between actors. The second is drawing attention to the complexity of relations as a result of this. SMEs are increasingly reliant on collaborative business efforts; whilst this opens up doors of opportunity, it further complicates stakeholder dynamics.

\section{8: Stakeholder Prioritisation: "Whoever is Banging at the Table}

\section{Loudest"}

\subsubsection{Preliminary Task Findings}

The focus groups included a preliminary task as a way of engaging participants and to initiate focus on the core topic of study. This activity required owner-managers to focus on key stakeholders, listing them in priority order. Time invested in nurturing stakeholder relationships is an investment and only likely to be pursued when stakeholders are key to the SME in some way. However, observations during the focus groups confirmed that the key stakeholders of SMEs vary considerably, with the prioritisation complex and context dependent. 
The preliminary task during the focus groups revealed personalised lists of key stakeholders in priority order for each SME; this was from the perspective of the owner-manager. The lists were complicated due to a number of reasons. The first of these was the personalised nature, with lists often including individual names of businesses or the same stakeholder classification at different levels of priority. The second was the different levels of power within stakeholder classifications that alter priority for the SME. For example, employees at management level on one occasion were prioritised higher than staff due to the power and business involvement differentials. Third, stakeholders prioritised were a reflection of the current circumstances of the SME and therefore the prioritisation of stakeholders is better considered as temporally mediated, context dependent, fluid and changing.

Stakeholders prioritised went far beyond those key stakeholders suggested by Spence (2016). These included employees, small local competitors, suppliers, local community, family and customers. Financial institutions, CSO's (civil society organisations) such as NGO'S (non-governmental organisations) and charities, local and wider government, education institutions, the media and business network facilitators were amongst other stakeholders named and prioritised in the focus group findings. This point will be discussed in greater detail in Chapter Eight. The following section will present the second aspect of the findings, the open discussion regarding the prioritisation of stakeholders.

\subsubsection{Open Discussion Findings}

As has been referred to in the themes above, CSR efforts for SMEs were reflective of the owner-manager's values and experiences. This could be an event in their personal life or those close to them. The personal nature of SMEs and their CSR efforts could be a reflection of family and friends being prioritised as stakeholders to the business, particularly evident when the SME was family led. Two SME ownermanagers display this below:

P18: "I immediately put down friends and family... without myself being well and without my family's support I couldn't do it”. (p. 2, $37-40)$ 
P13: "I've put family...because I have to it's a family business with them all breathing down my neck [laughter] ... with staff lower down".

(p. 1, 17-18)

However, it was employees and customers that were highest priority by the largest proportion of participants. This was often dependent on the context of the SME. For example, businesses that were skills based and reliant on their employees may be more inclined to prioritise staff higher than clients. Therefore, they may direct their CSR efforts towards these stakeholders, such as a focus on employee development and wellbeing. The following owner-manager explains the importance of staff for their knowledge-based SME:

\begin{abstract}
P21: "I've got staff at the top, we're a sort of staff based business, we're totally expertise based, what we sell is essentially our knowledge and experience and apply it to the client's situation, so without the staff we can't do anything, but it was a very close second with the clients because we wouldn't be able to do anything without them either". (p. 3, 73-77)
\end{abstract}

P12 confers with this notion suggesting that it is the people from within the business that are prioritised before clients:

P12: "The people that come before clients for us are the people within, I've listed them before the clients". (p. 1,9-10)

On the other hand, many of the SMEs explained that prioritising usually came down to the paying customers and clients that are essential for survival. For one ownermanager, they described their situation as being different to larger SMEs because of their sole trader set up, with a reliance on themselves and custom:

P16: "Because the situation's slightly different because they have quite a biggish company with other employees, then it's just me, so yes of course I'm very important [laughter] ... but you know from then everything comes from the clients". (p. 1, 12-15)

An owner-manager of an SME in hospitality concurs with the view that customers are the first priority: 
P8: "It's the customer who's coming through the door, they are the first priority". (p. 1, 5)

Furthermore, despite community commonly being prioritised after the key stakeholders mentioned above, the local community often encompasses those employees and customers. On one occasion customers were actually defined as the community of the SME. This has been demonstrated previously and it is evident that stakeholders are undeniably interrelated and overlapping.

Furthermore, P21 above brings attention to the necessity to coordinate multiple stakeholders in order to enable business operations. This was made apparent by the difficulty to discern which stakeholders were of most importance. Often it was a closely interrelated map of stakeholders working in unison that enabled the business to operate as a whole and not as mutually exclusive acts. The changing nature of stakeholder prioritisation is represented by $\mathrm{P} 1$ who explained that it is often dynamic, as represented in the dialogue below:

P1: "... who is banging the table loudest at any one time generally". (p. 2, 39)

This reflects the contextual nature of both stakeholder prioritisation in SMEs and the influence that this has on socially responsible activities. There was a multitude of different stakeholder groups and a number of contributing factors that influenced the prioritisation and salience of stakeholders. Urgency and proximity of stakeholders are two factors evidenced in the extract above, both elements that contribute to stakeholder salience. Again, these multiple contributing factors highlight the complexity of the relationships between SMEs, their key stakeholders and the implications this may have on driving CSR efforts.

\subsection{Barriers and Challenges: "A Big Change of Mindset"}

The complexity of stakeholder dynamics can contribute to the inhibiting of CSR engagement. Having already examined the drivers, it is not surprising that the focus groups also uncovered a number of challenges that SMEs face when pursuing their social responsibilities. Most commonly time and money were considered as major 
resource barriers. Subsequent challenges included moral deliberations for the owner-manager caused by proximity to stakeholders, CSR scepticism, higher demands from recipients of CSR, the escalation of requests, the cessation of CSR allegiances when associated with one actor in the partner organisation and finally, bureaucratic 'red tape' related to certain social responsibility initiatives. As P27 explains, due to these challenges it can be difficult for owner-managers to decipher what is actually the socially responsible thing to do:

\section{P27: "I think that sometimes it's difficult to know what is the socially responsible thing to do". (p. 6, 177-178)}

The resource constraints of SMEs are known and researched, it was therefore expected that cost implications would be a barrier for engagement in the short term. This is despite socially responsible initiatives often having long-term benefits. Lack of residual income for CSR was commonly indicated, with one owner-manager referring to CSR as a large cost for SMEs in comparison to large businesses:

\section{P27: "As a micro-business you see cost, all these things have costs.} Does there always have to be?" (p. 28, 830-831)

However, the most common barrier mentioned in the findings with regards to CSR participation was time as a resource. A lack of time was considered a major barrier, aligning to SMEs preference of resource-based CSR, such as the donation of expertise or skills. This was made apparent throughout the focus groups, with an example displayed below via a conversation between participants:

F1: "Are there any barriers that are stopping you from doing certain things that you would do otherwise?"

P1: "TIME [emphasised by participant]".

F1: "Time, definitely. Is everyone in agreement regarding the time aspect? [sounds of agreement]".

P6: "As a small business person, you don't have, you can't get 36 out of a 24 hour day, trust me, the work is there [laughter]". (p. 15, 425430) 
As is acknowledged by P8, time is a valuable resource and transfers as a net cost to the business:

P8: "It will cost you something, it is not free, so, when you do your budgeting for the next year, you've got to put some indication of time, whether it's in time or if it's in cash as well, but it does require some sort of source". (p. 15, 435-437)

It was rare that any of the participants allocated a set budget, however on the occasion below P21 allocated a certain amount of time per annum rather than monetary cost:

P21: "My annual time plan has got three weeks in the year dedicated to that, so again it's, well it's a net cost actually". (p. 14, 420-424)

He carries on to say:

P21: “...But no I get a huge amount back from it, it keeps me young”. (p. 14, 425-426)

P20 displays a view similar to many of the owner-managers of SMEs that socially responsible initiatives will be participated in unless it is of massive detriment to the business. This may be in terms of resources such as time and cost as displayed below:

P20: "What I will do, is do it unless it is of massive detriment to the company, so if there's, if there's no overall harm it will just be a bit of a pain, then even if it's got some cost to it". (p. 18, 553-555)

A further barrier identified by participants was a result of the personal nature of engagement. Often it is an individual that drives particular CSR activities, one owner-manager explains that due to this, if that individual leaves the recipient business or entity then this results in decease of such CSR allegiances and engagement:

P22: "We have some really committed groups but then it's usually an individual rather than the school so if that person moves on we have to find a new way in". (p. 3, 75-77) 
As a result, P22 is attempting to establish a commitment from the whole organisation:

P22: "One thing we're trying to influence is to make it a kind of commitment for the school or the business rather than just the individual so if that person moves on then they will make sure that somebody else takes it on". (p. 3, 79-81)

Further barriers with regards to individuals are the role of intrinsic emotions. The feeling of guilt often caused moral deliberations for owner-managers. The quote below shows how one participant resents feeling guilty if they do not support certain causes that approach the SME. The constant request for sponsorship was considered annoying considering that the SME already engaged with a number of causes per year, viewing it as unfair when stakeholders don't realise these associations and commitment to other CSR activities. The resentment of being made to feel guilty is evident in the quote below:

P7: "There are two very different aspects to this corporate social responsibility, one of them is the annoying constant request for sponsorship that we seem to get every day and it really is very frustrating. We have around four causes a year and that's it, but we don't want to be made to feel sort of guilty, because you know we've chosen ours, the thing I feel much more strongly about is the contribution of skills and time and that's something I've always been a bigfan of". (p. 9, 241-252)

A further intrinsic barrier for CSR engagement was scepticism towards the true motivations of CSR. This was derived from owner-managers' negative cognitions towards large businesses, tarnishing the concept of CSR. The reason for such scepticism is evident in the findings, with one participant explaining that their CSR partnership with a large charity was terminated due to increasing demands:

P6: "For the last two years we've sponsored a charity and a percentage of our income goes to them and unfortunately we sat down with them a couple of weeks ago and their demands are getting more and more".

P7: "Demands?" 
P6: 'Yes, they're wanting more and more money, and that's when we said no thank you, thank you so much but we're not interested, so it's interesting we've now gone back to supporting local mountain rescue which is actually putting money right back into the community, where we need it more, we really need it". (p. 9, 255-264)

Arguably this was not necessarily a barrier towards CSR because the SME redirected their charitable efforts towards a local charity, feeding back into the community where they felt it was more valuable. However, increasing demands are commonly occurring with regards to CSR partnerships, particularly with large charities, deterring engagement of SMEs when they cannot meet the demands. A further barrier to CSR is the increasing demands from stakeholders, discouraging SMEs from engagement. The extract below articulates that, with the communication of efforts, comes expectation:

\section{P27: "You can put it voluntarily. For example, put it on your website saying we do this, this and this, you're not paying someone to accredit you to do it you just put it on your website and actually, if you weren't doing it, people would complain because of the fact you're stating it'”. (p. 28-29, 839-842)}

P27 refers to the voluntary communication of CSR efforts on the SME website. The perception is that if efforts are communicated, expectations and scrutiny from stakeholders will increase and they will be held accountable to their claims. If these claims are not being adhered to then this could have negative consequences via word of mouth or loss of business. Consequently, increasing demands of stakeholders can actually create barriers for both CSR engagement and communication.

Close proximity to stakeholders is also seen to create challenges for owner-managers with regards to their CSR engagement. For example, one owner-manager recalls an example whereby their personal contacts with stakeholders of the business resulted in the owner-manager feeling obliged to adhere to CSR requests. This did result in the inception of CSR, but created moral deliberations for the owner-manager with unauthentic motivations for engagement:

P13: "I've just had the girls school netball team and I must admit I didn't really want to do that, but because her mum is on our Marie Curie committee I felt like I was obliged to". (p. 8, 218-220) 
An additional barrier mentioned by participants was 'red tape'. This could include regulations, paperwork or even the issue of measurement. For example, SMEs are stepping away from some CSR initiatives due to increasing amounts of paperwork that come with changing regulations such as health and safety. This is a barrier for SMEs due to their limited resources, as P15 explains, this may deter SMEs from taking on work experience students as one example:

P15: "A lot of companies that previously get involved with things like work experience, lots of businesses say that they've stepped away from that because of the changes in legislation and worrying about health and safety and changes". (p. 15, 395-397)

Finally, the lack of a common framework can also be a barrier to CSR inception and engagement. For example, there is no common language for CSR in SMEs. With scepticism towards large business CSR there are issues with aligning the conceptual understanding of the term. This is evident below:

P25: "There's also this wider issue of measurement, there isn't a
common framework that we all understand, because once you've got
a common language you can start to measure against that, but with
CSR it's so wide and with greenwashing it's really hard to say". (p.
12, 346-349)

In order to create a common understanding of CSR, accreditations are increasingly being introduced with regards to numerous forms of CSR. SMEs that take part in vending processes are now being required to present evidence of their social impact:

\begin{abstract}
P21: "It's the first time I've come across it actually as a formal process on one of those vender proposal questionnaires and I was personally very delighted to see it there, but wondered how other people would react because as I say, it is the first time I've met it and I'd be interested to see if it becomes a growing trend". (p. 18, 540545)
\end{abstract}

However, due to the cost and inaccessibility to SMEs, they may actually bridge an even bigger gap between small and large firm engagement with CSR. This is expressed by $\mathrm{P} 27$ below: 
P27: "I think I call it cost and unfair regulation currently is my view of it, because cost you know I've been told you have to get all sorts of accreditations now, which are huge costs for a micro business to prove that you are socially responsible when you are more socially responsible as small businesses". (p. 14, 397-402)

Despite the issues discussed above, P24 brings attention to what may be a larger societal issue. This issue is the attitude of society towards business. The current perception is that people only value things that can have monetary value put on them. As is explained below, this can be seen as a huge failing and requires a big societal shift in mind-set:

\section{P24: "There is a big sort of issue in our whole society in that we only value something that we can put monetary value on. So a business isn't doing really well if it's just about breaking even, but actually they've done all this good stuff socially. We don't value that at all and that's a big failing and somehow needs a big change of mind set". (p. 21, 612-617)}

To summarise these findings, there are commonly acknowledged resource restrictions for SMEs, with time and cost being major barriers. SMEs tended to participate in CSR so long as it was not detrimental to business. Additional points to take forward are first, the challenges of increasing demands from stakeholders and CSR collaborators. Moral deliberations are stimulated by rising expectations and proximity to stakeholders. The second is the bureaucratic red tape increasingly being imposed on SMEs. The lack of a common conception of CSR was considered limiting, yet SMEs were actively opposed to formalisation and rarely allocated budget for CSR. Finally, there is an issue whereby SMEs are not recognised for their social contribution because society is so focussed on economic rationality and monetary value.

\subsection{0: Economic, Political and Societal Influences: "Blurred}

\section{Boundaries"}

During the focus groups, attention was brought to the wider economic, political and societal influences that may shape SME owner-managers CSR orientation. In order to understand the way that SMEs make sense of CSR, an appreciation of the context 
allows a more detailed analysis of CSR on an organisational and individual level. This was evident in the focus groups whereby numerous context specific influences were referred to, for example, economic conditions, political structures and legislation.

Owner-managers believed that economic conditions often create challenges for SMEs. This can discourage engagement with CSR practises. A principal economic refrainment mentioned by participants was the high economic inactivity rate and areas of poverty within society. One owner-manager describes the economic situation below:

P22: "We have the highest economic inactivity rate, because while it's all very well talking about unemployment, actually economic inactivity is something like one in four people in Wales". (P. 23, 680682)

With P25 adding:

P25: "We've also got one of the highest poverty rates". (p. 23, 680687)

Economic issues are further made apparent when P27 refers to roads of empty shops. It is suggested that the only businesses surviving are charity shops due to the reduced business rates. The perception was that the council will not reduce business rates for SMEs and would rather allow the community to die. Economic issues partnered with a perceived lack of political support from local councils are evident in the extract below:

\footnotetext{
P27: "You see road after road where all the shops are closed or empty. They're either closed and empty or they're charity shops and the reason for that is that charity shops don't pay business rates and there is no reduction, even if you've got a whole road of empty shops the council won't reduce the business rates. So they will let that community die because they won't have reduced business rates". (p. $25,731-735)$
}

Such economic conditions can influence the focus of SMEs, with emphasis being on the economic viability of the business and not social responsibilities. One owner- 
manager proposes that SMEs embedded within high performing local economies are more readily able to engage in CSR, and therefore are not actually providing to the communities that really need the assistance in the first place. For underperforming local economies, SMEs may not have the capital, capabilities or resources to mobilise and engage with CSR to assist their community and improve the economic position. P15 expresses their concerns in the extract below, explaining that high performing local economies can form the voluntary precept and therefore they don't need assistance in the first instance:

\begin{abstract}
P15: "My concern is that only businesses in good, high performing local economies can form the voluntary precept, therefore they don't really need it anyway, because the economy is already doing well. That's on the side but at what point, I guess does a commercial decision become a social decision? You know there are boundaries I guess that are blurred". (p. 24, 710-715)
\end{abstract}

From a different perspective, P18 believes that closeness of communities can create solutions to overcome difficult economic conditions, driving socially responsible initiatives and aiding survival. P18 recounts a localised effort to maintain Scouting and Guides to support disadvantaged children of the region. This was extremely effective for both the businesses and local area.

P18: "I'll go back to the scout post because that was so remarkable, it actually kept scouting and guiding alive through very hard financial times, for some of the scout groups especially in the poorer parts of [Location 3] it was their only income. They could not get subscriptions out of the kids because the kids have no money, so the only thing that actually kept them alive in those very deprived communities was the scout post and that in its own right is quite an achievement and it was the local shops and local people supporting their local groups". (p. 10-11, 313-320)

The owner-manager put this down to closeness to the community, direct relationships and face-to-face interactions, as is seen below:

P18: "There was no big umbrella organisation, it was you know the scout group at the end of the street talking to the shops at the end of that street and in that area and having your direct relationships". (p. 10-11, 320-322) 
P18 further emphasised the importance of localised and scaled efforts for CSR engagement in the extract below:

P18: "Localised efforts are very very affective and I think it's all about scaling them". (p. 10-11, 328)

Despite the negative perception towards local authorities and the rigidity of business rates, the Wellbeing of Future Generations Act (2015) has recently been introduced to Wales in the UK. Despite this policy specifically targeting public bodies, the act will commonly be referred to and impact SMEs, with the aim to raise all businesses up to a minimum standard. The request for social impact evidence in public sector tenders is one example of the impact on SMEs. P25 expressed below their happiness that CSR is being introduced within tender submissions:

P25: "CSR could make a big difference in what I do and I've never been asked to codify that in any tender I've submitted and by the way the tenders I go for are always with Welsh Government".

P23: "I think you'll see them referring to the Wellbeing of the Future Generations Act in the future".

P25: "I hope so. I really hope so".

P23: "Anybody heard of that? It came into force on the first of April, so it's seven goals that link to the sustainable development goals so you're going to start to see more questions of 'How are you contributing to this?' It's supposed to influence our decision making processes, I mean businesses aren't covered by the act it's only really public bodies, but there is an expectation that everyone, from us I think to make sure we're evidencing it in the decision making process. That is what you do without even knowing it".

P27: "From what we're hearing it's what everybody is doing here anyway”. (p. 11-12, 312-333)

Findings further show support from the Government, with current structures in place to address unemployment and aid economic development. The owner-manager below assures peers that schemes such as Jobs Growth Wales are of benefit to SMEs and aid prosperity. Assistance from the government such as this will aid SME survival, growth and potentially allow them to re-direct more resources towards 
socially responsible activities. The owner-manager below recounts their positive experience of Governmental assisted schemes:

\begin{abstract}
P14: "It was just me, myself and I for the first year and then coming up to twelve months worth of trading it had to expand but then money was still tight so the Jobs Growth Wales Scheme sort of ticked all the boxes for me in terms of getting someone young back into employment... and having that six months worth of subsidised wages is a big help when you're a small company. Yeah the Jobs Growth Wales Scheme has been hugely important for us." (p. 3, 62-70)
\end{abstract}

P20 further supports the positive influences of Governmental funded schemes by stating that their SME would not have survived without the support:

P20: "As a recipient of Business Wales support which is government funded, I wouldn't be here without it".

P17: "But that is tax payers money, not just government".

P20: "But it's the government doing it, because that tax payers money doesn't have to go to that cause so it's not just the government massaging figures and saying look what we've done, it's the funding of schemes they've provided". (p. 16, 484-489)

On the other hand, P24 explained more precisely the limitations of certain governmental initiatives in her experience. She clarifies that SMEs, as her client base, are often not eligible for support because they are not 'high growth'. SME start-ups are often a lifestyle choice; consequently many do not actively pursue growth, with the emphasis instead on surviving and maintaining their current position. Consequently, as P24 makes apparent below, such initiatives are not accommodating for a large proportion of SMEs:

\title{
P24: "There are some Welsh Government business advisors, although they've stopped advising my client base which I'm very cross about because they're not growth businesses, so they don't get any help anymore”. (p. 2, 53-55)
}

She further proposes that policy makers need educating about the needs of SMEs. It is suggested that sometimes advice is misinformed and not in the best interests of smaller businesses: 
P24: "The policy makers definitely need educating about lots of things in business, I think I've stopped a few of my businesses only just in time from making stupid decisions because they, the Welsh Government supporters have sort of said 'Have one of these apprentices, it's funded by us it won't cost you anything'. They don't think about employers liability insurance, they don't think about the cost of adapting the premises for a second or third employee". (p. 15, 448-455)

\subsection{1: CSR Awareness: Disseminate and Educate}

Educating and the dissemination of knowledge was a common topic of focus groups. SMEs were seen as the greatest resource to assist, disseminate and address knowledge gaps for new start-ups and fellow SMEs. Many owner-managers saw it as a duty to educate in the form of apprenticeships and mentor programmes; P21 expressed the importance of passing on expertise and described his plan to transfer pockets of knowledge to different recipients. One way of doing this was as an industrial mentor for University students. This simultaneously contributed to his social responsibilities as an owner-manager:

P21: “I don't want my expertise to fade away with me, so I've got a personal plan to transfer packets of it to different people over the next ten years or so". (p. 14, 416-418)

As mentioned, SMEs are a vital resource to assist other SMEs. P25 suggests that this is because people are more likely to trust peers and engage with those that aren't wearing a white coat:

P25: "You learn more and trust more from your peers, and I think that's true in what ever walk of life, you learn more, you trust more, you'll engage more with someone who isn't wearing a white coat". (p. 26-27, 784-786)

Additionally, P18 advocates business owner-managers as the greatest and most accessible resource for new start-ups in the following extract:

P18: "I think that in my experience, existing business owners are the best [emphasised by participant] resource and the most likely 
resource that's accessible to start ups, to help and educate, to mentor, to support, to give them opportunity, to direct them to opportunity streams". (p. 16, 474-479)

With regards to CSR, this is influential as existing SMEs could be a vital resource to create awareness of the social responsibilities of SMEs. P8 acknowledges that as the focus group attendees are active members of the FSB, they are more likely to be proactive and have a more comprehensive understanding of the CSR concept. The challenge underlined by the following recipient is the ability to reach the SMEs are not aware of CSR:

P8: "We're a round table of well educated people sitting here, we have a fairly good idea of what CSR is, what we're talking about is CSR in small and medium-sized enterprises in Wales so it's a question of reaching the parts who are not even aware of what it means and to educate those people to take a go at what we mean by CSR”. (p. 11, 315-320)

Some participants had already made steps to disseminate their knowledge on the benefits of sustainable practices. One owner-manager described the adoption of green initiatives and zero waste as their unique selling point. The initial motivation for this CSR engagement was the cost savings on a 'lean not green' agenda as described below:

P6: "It's very relevant to us because it is probably our unique selling point you know. We based our business around green initiatives and yeah I would like to say that we do very nicely out of it. But as people know around the table, the way we came to it was on a lean not green agenda". (p. 6, 164-167)

However, the owner-manager viewed it as a duty to educate on the importance of sustainability for business and society at large. When asked of the appropriateness of sustainability, P6 responded with the following:

P6: "There is no two ways about it, sustainability is real, very much part of our business and we try, on the softly softly approach to educate people as we go... we do try and influence them by the practises that we have". (p. 6, 172-174) 
Nevertheless, the focus groups revealed challenges with regards to the communication of CSR efforts and the potential for dissemination of knowledge. The majority of the owner-managers were not interested in promoting their CSR efforts. This was evident in the comments below when they were asked if they expected anything in return for CSR efforts:

P13: “...we don't get any publicity and I don't really want it”. (p. 8, 208)

P14: "Yeah, we prefer to be like P13 and stay as anonymous as possible”. (p. 12, 313)

P9 explains the lack of communication, suggesting that SMEs often do not realise the extent of their positive contribution to society and therefore are not recognised for the good work that they are involved in:

P24: "Little businesses that I've mentioned before do lots of good stuff, but are absolutely not recognised for what they do and don't promote it and don't shout about it and to be honest don't even realise they're doing anything that's good. They could make something out of it but they don't'. (p. 31, 924-927)

P8 proposes that there is still a long way to go with regards to spreading awareness of the CSR concept. A suggested solution is to use events such as the focus groups to disseminate such knowledge:

P8: "I think we need to disseminate what we are proposing here to the wider audience because we are all practitioners and there is a need for more dissemination of events around Wales for SMEs to raise their awareness of CSR. That is important. There are a lot of willing people out there but they just don't know it". (p. 20, 582-585)

Finally, participants not only reflected on the importance of educating individuals and businesses, but also considered the importance of shifting societal perceptions. The value of educating and nurturing younger generations with a mind-set not solely focussed on economic rationality was evident. In the following extract, P24 suggests that younger generations already seem to be displaying more of a social consciousness that accommodates CSR, incentivised by morals and not just profit: 
P24: "Young people seem to be less profit driven and more driven by other things so I don't know whether that just means that there is hope or if they're being educated better, or that's just something that we can work on, I don't know. But they seem to be more motivated by environmental concerns, social concerns, I don't know...more so than the sort of the Thatcher generation". (p. 42, 1241-1246)

This theme brings attention to a number of points that deserve additional focus. A key finding is SMEs as a resource of knowledge for other businesses. An interesting discovery however was the challenges of communication and the dissemination of such knowledge. These challenges could be inhibiting the distribution of information on CSR and contribute to the lack of awareness and unified understanding of the concept. A further challenge is the undervaluing of SMEs perceived impact on society. The dissemination of CSR awareness and value of CSR for SMEs and potential recipients is a theme that merits further development. This aligns with the following sub-section.

\subsection{2: CSR Impact: "The Planet and the People"}

The final theme derived from the focus group findings was the nature and impact of CSR efforts. Owner-managers commonly viewed CSR as a large firm, tick box exercise. There was a consciousness that CSR efforts should instead be considered with more detail to ensure that they are sustainable, provide long-term solutions and actually contribute to the enhancement of societal wellbeing. The discussion below identifies the hostility towards some current examples of seemingly unsustainable CSR:

P24: "CSR could be better directed I think, just as an example Tesco repainted a community centre in [Location 4] in Tesco colours you know and"...

P25: ["Surprise, surprise"].

P24: "... and the trouble is the community centre is still going to close so it hasn't solved anything and I think that money would have been better spent in some other place”. (p. 6, 161-167) 
It is proposed that large firm CSR would be more affective if they worked in collaboration with local communities:

P23: "Yeah and perhaps working in partnerships with local towns and its communities where they operate then their corporate social responsibility might work. But at the moment it makes it look like they are greenwashing”. (p. 6, 170-172)

It was the tokenistic nature of engagement in larger organisations that facilitated the perception of greenwashing identified in the extract above and below:

F1: "There seems to be a little bit of hostility towards the larger businesses and the way that they deal with CSR. Am I right in thinking that? Does anyone think that they actually contribute positively?"

P22: "It often seems tokenistic, just to tick a box so". (p. 33, 979982)

P23 suggests later in the conversation that CSR should instead be integrated as a concept into the core of businesses, ideally from start up:

P23: "I think it's about getting them to think the other way around, so if they looked at their core business like you do when you're setting up, look what are you here to achieve and get it right from the beginning rather than trying to fix it”". (p. 33-34, 989-994)

Such scepticism of CSR was common, viewing large firm CSR efforts as empty and solely for instrumental motives such as public relations and the bottom line impact. P17 expresses the view that CSR itself needs to be sustainable; with a long-term perspective to ensure it is benefitting the recipients and society:

P17: "I agree that these people are doing some great stuff to help, well that's great and is their CSR, but that in itself is not sustainable in the longer term, back to my earlier point about the planet and the people. I think you need to understand and businesses need to understand that citizens need to look more closely at who is having what, on what basis and is your model a good model because are you really helping people?" (p. 10, 291-297) 
He expands on this view by referring to the previous theme of personal responsibility. The sustainability of such initiatives and potential strategic impact are considered as a responsibility of the individual as well as the SME as a whole:

\section{P17: "You have to be personally responsible for CSR when you give it, just asking questions. 'Who is it actually helping?' 'Is it helping the people that I feel I want to with my business?' You know, rather than just ticking a box we do need to all become more aware of that". (p. 10, 301-304)}

In summary, the need for SMEs and their owner-managers to consider the value of their CSR efforts in the long term was evident in the focus groups. Tokenistic and instrumental CSR in the past has resulted in scepticism towards the true motives for businesses engagement. In order for SMEs to counteract this perception of the concept, CSR needs to be embedded and sustainable in order to contribute to the improvement of local communities and society.

\subsection{3: Chapter Conclusion}

The conducting of focus groups was beneficial to refine the research approach in terms of both data collection and methodology. Methodologically the social constructionist approach was confirmed as appropriate, reinforced by the complex and diverse nature of CSR engagement discussed. It was also apparent that there was ambiguity and a lack of understanding of what the term CSR represents conceptually for SMEs. It was evident that more detailed and in-depth exploration was needed, leading to the selection of semi-structured interviews for the following phase of data collection. A number of themes emerged from the focus groups that helped refine the focus of this second phase, informing the questions thematically in order to guide interview discussions.

The first theme identified was the discursive ambiguity for CSR. Although the concept is being practiced within SMEs, the word corporate was still considered excluding, despite increased exposure of the label nationally. SMEs displayed a disassociation to the term due to conceptual disparity with the large corporate perspective of CSR as an instrumental activity. Alternatively, a diverse vocabulary 
was used to encompass the concept. When labelled anything at all, CSR was most commonly socially constructed with reference to moral responsibilities, the notion of giving back, or with reference to community relations. A lack of conceptual consistency is a limiting factor for SMEs; a drive towards unity of CSR terminology in SMEs may facilitate awareness of their conception of CSR and a re-moralising of the concept. A more specific exploration of the use of language and conceptualisation of CSR in SMEs would prove interesting.

Individual actors and their implicit orientations play a large role in the inception and engagement of CSR in SMEs. In much of the dialogue, CSR was considered a personal responsibility of the owner-manager and therefore an embedded part of their SME. Their perception was that as individuals, they are more intrinsically engaged compared to large corporates. This may explain in part why SMEs disassociate with the term CSR, as an attempt to reposition efforts as driven by more genuine motives. The permeable boundaries of business and personal spheres mean that SMEs cannot be seen as isolated entities or separated from the individual actors within the business; in fact, CSR is often directed towards causes, initiatives or groups that have personal significance. An appreciation of the integral role of the owner-manager will therefore be vital to capture the complexity of CSR in SMEs and understand the way that it is made sense of.

However, CSR inception is not only driven by owner-managers within the SME, but also prompted by a diverse range of stakeholders or circumstances external to the business. Key stakeholders were unique to each SME and dynamic in priority, reflective of their current circumstances. Furthermore, there was often a reliance on closely interrelated webs of stakeholders working together to mobilise SME operations. The inception of CSR was therefore commonly stimulated by requests filtered through close stakeholder networks or internal actors and motivated by the need for relational development. This closeness to stakeholders can be beneficial for SMEs, with CSR enhancing relationships via communication mechanisms such as word of mouth, increasing reputation and legitimacy. However, it also intensifies pressure for SMEs to adhere to the increasing demands of stakeholders, causing moral deliberations for owner-managers and inhibiting factors for CSR uptake. 
Proximity and partiality towards close stakeholders is therefore something that warrants further exploration in the next phase of data collection.

Owner-managers commonly referred to community in the way that they socially construct CSR. SMEs are characterised by their embeddedness within communities, with such closeness acting as a core catalyst to create webs of stakeholder relations to facilitate their survival. Closeness to the community on a personal level meant that CSR efforts for SMEs were often directed by a sense of duty to enhance and service them. What seems apparent is the importance and value of SMEs to their local community, not only for economic prosperity but to encourage sustainable social prosperity in localised regions. However, the role of physical communities may be changing for SMEs with the rise of technological developments and e-commerce. The implications of this on the dynamics of SME operations and their perceived sense of responsibility towards close stakeholders is thought provoking.

SMEs cannot be separated from their context. The fifth theme regards the societal landscape of the SME as a crucial factor to understand the way that SMEs make sense of their social responsibilities. Participants referred to economic conditions, political structures and cultural factors that all influenced the role of CSR for their SME. These wider conditions acted as either inhibiting to the adoption of CSR, or positioned CSR as a solution to overcome wider societal issues. However, a key point to take from this theme is the perception that there needs to be a shift in mindset of society from an emphasis on profit and economic rationality to considering the long term impact of decisions on society with regards to social, environmental, cultural and economic wellbeing. The introduction of the The Wellbeing of Future Generations Act (2015) formalises this need for public sector organisations and is a start to the enormous attempt to re-moralise and shift the overall societal mind-set.

The final theme regards the dissemination of knowledge and the importance of creating awareness of the collective contribution SMEs make to societal wellbeing. Owner-managers perceived it as a duty to pass on packets of knowledge and spread awareness of the need to be socially active as a business. They also considered themselves as the greatest resource to assist new start-ups and fellow SMEs. The 
challenge expressed is reaching and educating those not engaged with the concept already, with this exacerbated by some SMEs reluctance to communicate their CSR efforts more widely. It also deters the dissemination of knowledge on the topic of CSR; therefore efforts are needed to spread awareness and gather appreciation for the unique way that SMEs make sense and engage with their social responsibilities.

To conclude, it is clear that CSR in SMEs is complex and multifaceted in concept and practise. SME owner-managers scepticism towards the concept has been unearthed due to the large firm conception of CSR as profit driven and a tick box exercise. It was made obvious that there is a current need for responsible CSR engagement and suggestions have been made that individuals should carefully consider the long-term impact of their CSR efforts, to ensure that they are sustainable, actually directed towards helping the recipients and providing solutions that contribute to overall societal wellbeing. Owner-managers of SMEs made explicit their view that CSR should be integrated into the core of business activity, with individual actors taking responsibility for the overall ethical character of the SME.

The following chapter will explore in more detail how SME owner-managers make sense of their social responsibilities and the role of stakeholders within their context specific environments. A selection of themes from these focus group findings will be taken forward in order to understand the appropriateness of moral perspectives to explain CSR in this context. These themes include the following:

- Prioritisation of responsibilities

- CSR terminology and conceptualisation

- Owner-manager values

- Key drivers and motivations

- Stakeholders

- Closeness to stakeholders (proximity)

- Community

- CSR engagement

- Barriers and challenges of social responsibility 
- Measurement and formalisation of social responsibility

The focus group findings have provided an open and multi-layered exploration of the CSR phenomenon in the SME context. However, for the purposes of this research, moving forward the interview questions will focus more specifically on the individual and organisational level. 
Figure 6.1: Summary of the Interview Themes
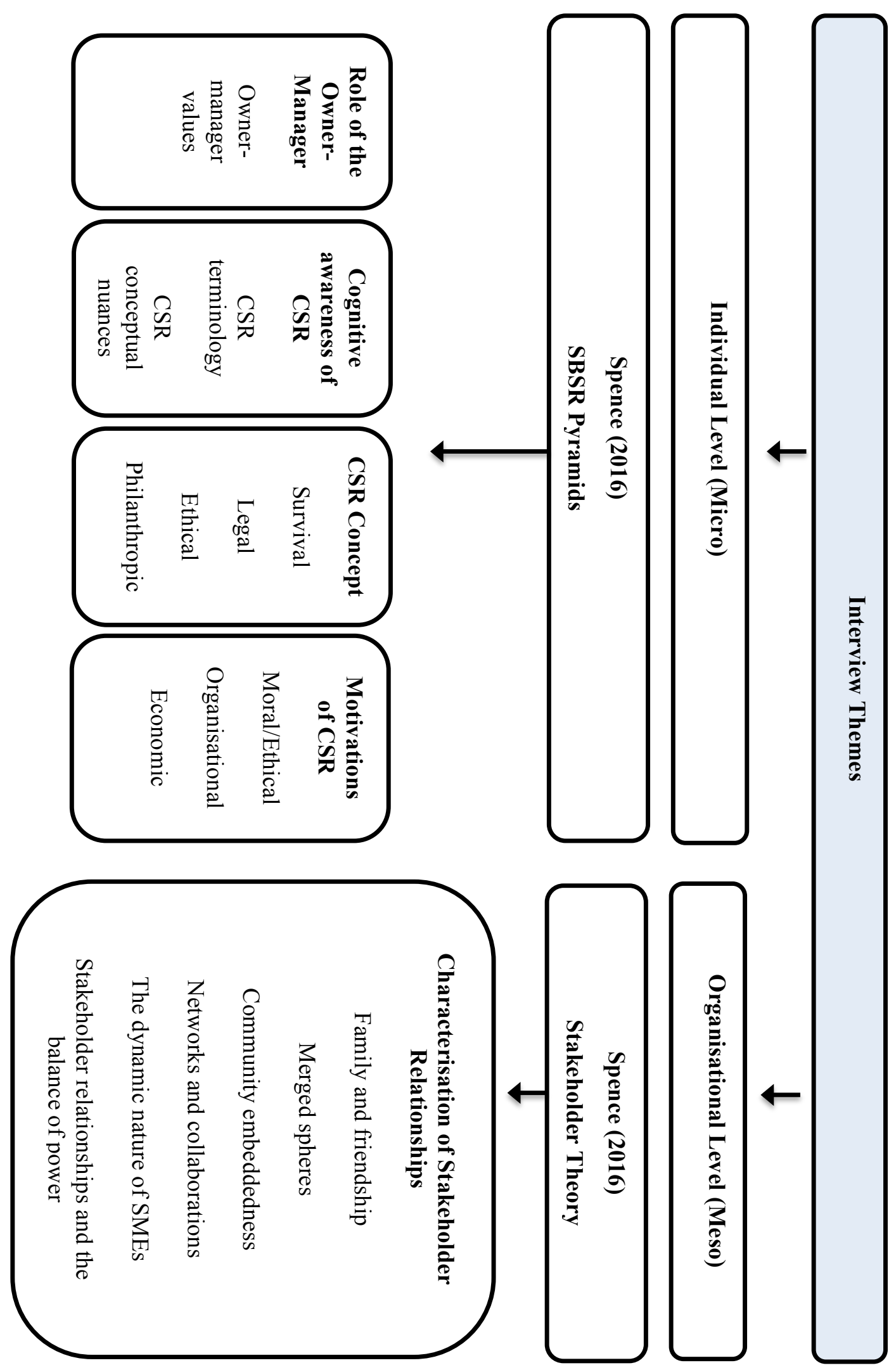


\section{Chapter Six: Interview Findings (Micro - Individual level findings)}

\section{1: Introduction}

The two following chapters will provide the results and interpretation of the interviews as the second stage of data collection. This chapter will specifically focus on findings at the individual level of analysis, providing evidence to evaluate the appropriateness of the redrawn SBSR pyramids proposed by Spence (2016). This is by concentrating on the role of the owner-manager and their conceptualisation of CSR. The full justification for the second phase of data collection can be seen in Chapter Four, but to reiterate, this was a valuable step to explore a selection of super-ordinate themes in greater detail. By providing focused and rich detail on certain themes, it allows a more direct approach to make sense of the complex data and to answer the research questions.

Whilst the interview analysis was broadly inductive, it was informed by a priori assumptions, denoting reasoning from existing theories, models and frameworks. The structure of the analysis therefore draws from the likes of Carroll's (1979) CSR pyramid, Spence's redrawn CSR theories $(2014,2016)$ and the CSR perspectives from Wickert et al. (2016). These helped to refine the structure of the findings in order to address the research questions. The super-ordinate themes are therefore influenced by the key research questions (see Table 1.1), focus group themes (see Appendix 4.10), relevant literature and emergent interview findings (see Appendix $6.1)$.

This refinement does not mean that wider influencing factors are ignored, but that close analysis of all themes is beyond the scope and time frame of this research. It is further acknowledged that the different levels of findings are not mutually exclusive, but integrated and iterative in their implications. The rest of this chapter is organised into five super-ordinate themes (see Table 6.1) that structure the analysis. These are divided into a number of sub-themes that guide the content within each of the headings, as displayed in the table below and in Appendix 6.1. To conclude the 
chapter, the findings are summarised and attention turns to Chapter Seven to deal with results that pertain to the organisational level to help address the appropriateness of Spence's (2016) redrawn stakeholder theory.

Table 6.1: Interviews: Summary of Super-Ordinate Themes for Individual Level

\begin{tabular}{|c|c|}
\hline \multicolumn{2}{|c|}{ Individual Level } \\
\hline Super-Ordinate Themes & Sub-Themes \\
\hline The Role of the Owner-Manager & $\begin{array}{l}\text { Owner-manager morals, } \\
\text { characteristics and values }\end{array}$ \\
\hline Cognitive Awareness of CSR & $\begin{array}{ll}\text { - } & \text { CSR terminology } \\
\text { - } & \text { CSR and conceptual nuances }\end{array}$ \\
\hline Conceptualisation of CSR & 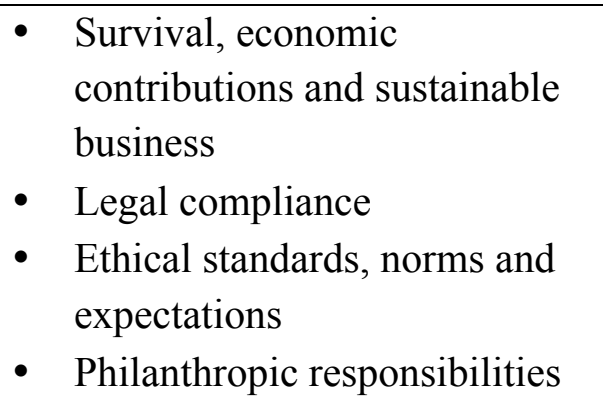 \\
\hline Motivations and Drivers of CSR & $\begin{array}{l}\text { - Intrinsic motivations and moral } \\
\text { obligations } \\
\text { - Organisational motives and } \\
\text { relational drivers } \\
\text { - Instrumental drivers and } \\
\text { tangible outcomes }\end{array}$ \\
\hline
\end{tabular}

\section{2: The Role of the Owner-Manager}

This section considers the role of SME owner-managers in CSR, a theme derived from the focus groups that deserved further attention. In order to identify how SME owner-managers make sense of their responsibilities towards society on an individual level, the following segment provides evidence with regards to their morals, characteristics and personal values. Results that attend to the research objective are presented and analysed using verbatim quotes. The results highlight the important role of personal values in the social construction of CSR. They further feature a direct influence of the individual on organisational level factors such as 
stakeholder relationships and engagement with certain forms of CSR.

\subsection{1: Owner-Manager Morals, Characteristics and Values}

Similar to the ideology of social responsibilies present in the focus groups, the importance of individual responsibility and personal values of the owner-manager was emphasised as an integral pillar for being socially responsible in SMEs. Many participants highlighted their personal integrity and morals as the invaluable foundations which their business and reputation is built upon, as evidenced by P16 below who described the SME as an extension of herself:

P16: "Personal integrity, you just can't buy reputation and that's one thing I will say a lot, you just can't and I think over time you earn that kind of respect and you get that reputation through working hard and yeah, you can buy a lot of things but you can't buy that and I think that's what I build my business on, so I suppose it is an extension of me". (p. 3, 77-81)

As P10 explains below, responsibilities for SMEs are still very personal and behaving responsibly is as much about personal reputation as it is business:

P10: "Going back to your point about you know, responsibilities, you can overcook it really. It's still very personal. It's all about giving good service and reputation and that sort of thing”. (p. 27, 738-741)

Personal values such as integrity, honesty, fairness, reputability and trustworthiness were dominant themes stressed as invaluable by owner-managers. These personal values and the way in which SME owner-managers make sense of their responsibilities can be seen as merged into business relationships on an organisational level. For example, maintaining long-term relationships with stakeholders perceived to have similar morals aligned with the vision of the ownermanager and SME. This is seen in the extract below:

P4: "Yes, all the people I'm working with now I've been working with for a long time, so these are people that have been there pretty much since the beginning or there or thereabouts. They're ten plus years of continued service and I think they're really good humans, you know 
they've got good morals, they're really hard working and they believe in what we're doing". (p. 11, 307-311)

Already it is evident how the personal values of individual actors are influencing stakeholder relationships on an organisational level. P29 further identifies how moral and ethical alignment and shared vision can assist in the obtainment of new customers. This acts as a bonding mechanism and reminds us that the human element still matters in business:

P29: “...they were looking for somebody who was morally, ethically aligned with them as a business...that's what they wanted, they look for people rather than...they do look for skills obviously, but they want people who fit their business so they met me, they liked me, so I was just stuck with them for about two and a half years now". (p. 12, 345-349)

There was a further understanding amongst the majority of SME owner-managers that the preservation of reputation and trust were invaluable for both personal and organisational spheres. Such values were seen as outcomes emanating from the personal morals of owner-managers and application of those to the responsible and ethical management of the SME. Long-term business survival as an economic responsibility was associated with such ethical and responsible behaviours. The following quote demonstrates this point:

P10: "We try to operate in a very ethical and responsible way I think and you know, we want to be here for the very long-term, as long as we can possibly be, so we try to preserve our reputation that way and behave in a responsible and ethical and a fair way. We do take it very personally. If we think we've treated people badly we naturally want to put it right and to the best of our ability and we're very happy to put our hands up and acknowledge that things haven't gone too well. I mean, hopefully that's not too often, but that's I think hopefully part of our you know, responsible trading pattern as well really". (p. $1,17-25)$

These few quotes alone provide evidence of the unique way in which SMEs make sense of their responsibilities towards society, with the individual and personal influencing and merging with the business. Consequently, responsible business behaviour broadly speaking can commonly be seen as a reflection of personal characteristics and beliefs of the owner-manager. 
However, to acknowledge owner-manager values as the only significant contributing factor for CSR would be a misrepresentation of the complex nature of CSR inception and engagement. When it comes to the inception of CSR within SMEs, the influence and pressure from complicated webs of stakeholders plays a vital role in determining ultimate CSR engagement. This is further complicated due to the embeddedness of SMEs within forms of community and consequent proximity to stakeholders.

The above point with regards to the influence of stakeholders will be discussed in more detail in Chapter Seven. However, one important point to take away from these findings is the personal element of SMEs that influences organisational level factors, ultimately impacting the uptake and nature of CSR.

\section{3: Cognitive Awareness of CSR}

Having briefly analysed the role of personal values on the construction and engagement of CSR, this section turns attention to the owner-managers cognitive awareness and conceptual nuances of the term CSR. This is to uncover how ownermanagers make sense of their responsibilities towards society through the language that they use.

\subsection{1: CSR Terminology}

The acronym CSR was purposefully omitted in the early questions of the interviews in order to gain an accurate representation of the terminology used for the firms' social responsibility initiatives. There was some definitional ambiguity when participants were asked if they understood or used the term 'CSR'. The majority of participants used alternative terms if anything at all for their CSR efforts. These involved words such as donations, helping out, social value, giving back, social responsibility, charity and most commonly terms involving community. The spectrum of CSR terminology ranged from absolutely no awareness of the term as below: 
I: "Okay. Do you think that the term corporate social responsibility is suitable to describe what you do? Do you even know what that means or do you feel it means something else?"

P12: "What did you say it was called?"

I: “Corporate social responsibility”.

P12: "I don't know what that means". (p. 15-16, 394-399)

To people who understood the concept of CSR, but called it something that they deemed more appropriate for their SME, as seen in the two separate extracts below:

P29: "The term does sound quite alienating, but I get what you mean by it and I think if it was called something nicer, it would sound better, but it is, in effect, that though, isn't it? Giving back, regardless of how small or wide the community and how big or small the company". (p. 22, 610-613)

P27: "Yes, the government has guidance for local authorities and public sector bodies on social value in procurements, so it does tend to feature. You always get a social value question. So whether that's providing work experience, whether that's providing staff training to up-skill your existing workforce. So all of that would come under CSR”. (p. 20, 549-553)

On the other hand the term CSR as a term was seen to be gaining some traction. This was particularly the case for those SMEs having to tender for jobs, as seen above. It was also common for SMEs to use CSR as a term when working closely with larger organisations that have adopted it, as demonstrated in the following extracts:

I: "Do you understand CSR and what it means?"

P4: "Yeah, yeah you know some of the bigger clients I've worked with in the past they've used those terms and they've done particular things that would definitely come under that category". (p. 15, 423426)

P2 has also adopted the term because of the exposure in the industry of which the SME is situated: 


\title{
I: "Do you use the term CSR or do you use anything else?"
}

P2: "We, we would use it because our clients use it so it's something we're quite familiar with, it's almost as if it has it's own discipline in engineering recruitment". (p. 9, 227-228)

However, there is still an element of disassociation with the term CSR despite the acceptance of what it constitutes. One owner-manager considers the use of CSR important in terms of consistency, so that everybody would know what it was and what it means. However, the same owner-manager claims that the documentation of it could and would be considered pretentious:

\begin{abstract}
P18: "The old buzzword generator, you do have to have consistent use of terms so we all know what we're all talking about um, yes I have a formal health and safety requirement plan because the law requires me to have one, I don't have a corporate social responsibility document because it would be pretentious and I've got better things to do with my time than write it but if I made one, I'd call it that because everybody would know what it was and what it meant”. (p. 7, 205-210)
\end{abstract}

In summary, CSR as a term has not gained widespread adoption, despite increasing focus on the relationship between business and society in business, policy and academia. There are still SMEs that have not heard of the term before, but most commonly the concept of CSR is renamed to language more suitable to the specific SME, often referring to community engagement of some form. The findings do seem to imply an incline in adoption of the term due to exposure from large corporates and inclusion in procurement documents, yet there are still diverse interpretations as to what it may constitute as a concept. These conceptual nuances will be discussed below.

\subsection{2: CSR and Conceptual Nuances}

The owner-managers of SMEs in Wales exhibited diverse perceptions of their role and responsibilities in society, with varying levels of awareness and understanding as the below quotes illustrate: 
P2: "I mean our main responsibility is to earn money and that, that is the number one thing so we are focussed more than anything on just doing the business that generates revenue, but at the same time probably secondary to that um, it's making sure people enjoy earning money”. (p. 1, 19-22)

P6: "Yeah as a community interest company it really does sum it up, in that we're just out there for the interests of the community and we're out there for the sake of the community". (p. 14, 415-417)

The perspectives of CSR revealed a conceptual continuum from those who equate it simply with ad hoc charitable giving, to those who considered it strategically in terms of sustainable, long-term development. The quotes below demonstrate this contrast in CSR perceptions, first from an SME owner-manager who perceived CSR as purely charitable:

P4: "I mean yes it describes it, but I wouldn't say that it would be a commonly used term for businesses like mine you know, we do it because it makes us feel good and warm and fluffy inside, it's not a kind of corporate speak that we would apply, no-one would say that in the business we'd just say for example we're helping a charity". (p. $14,410-413)$

To an SME owner-manager who perceived CSR as much more than just charity, displaying a more comprehensive understanding of the complexity of the concept:

P20: "I don't think that CSR gives it enough... it doesn't, when you say corporate social it doesn't pull on my heart strings or describe everything that comes under the term. Because you are not just saying you know, giving back or being charitable but community work and the long-term development of employees. I say in my video...I did a video on what it is because CSR has a massive, massive, massive scope because you can go into sustainability and recycling as part of CSR, being green”. (p. 15, 414-420)

It may be worth noting that despite many of the SMEs not engaging with the term CSR or articulating a comprehensive understanding during the interview, their actual CSR engagement was advanced in terms of addressing the long term needs of others within society. As P8 explains, often there is a disassociation with the word 'corporate', yet CSR it is still going on without the owner-managers realising: 
P8: "I wouldn't call it corporate, because again I guess when you're a small business you don't really think of it, but it definitely goes on I think without even realising it'”. (p. 18, 459-460)

The following is an example underlining the reason for this disassociation, whereby P21 explains that the term CSR is not one that they feel aligned with. This is due to the large firm associations, despite the underlying principles and concept of what they are doing being the same:

P21: "When you hear the word 'corporate' it's not a word that I feel aligned with, because it feels like it's for a bigger organisation, that's how I see that word. But I get that in the principle of...yes what it's doing is actually...yes it's probably what we're doing isn't it?" (p. 15, 413-415)

An example of this nuance in the labelling and conception of CSR is presented by an SME below. Despite not giving any thought to what their socially responsible efforts should be called, their actual CSR engagement was advanced in terms of providing sustainable support that will contribute to society in the long term. This is evident in the proceeding extracts:

P21: "I've set up a project for refugees and vulnerable women, they're coming here and we're making jewellery and fairies and my idea is to set up a business that they can make money from, that's the plan". (p. 19-20, 523-525)

She continued to say that she wanted to help them into employment:

P21: "I think that could be really good and just help them with what they want to do with their lives and get them into employment. I've got three Syrian refugees and they really want to sell food like cooking, like falafels and stuff like that, so I was just phoning the Environmental Health to try and get them their kit, to get the Environmental Health to come out and look at their kitchens to see what they need to do to make it safe so that they could start cooking and selling to earn a living". (p. 20, 531-536)

Despite a large proportion of CSR engagement described as being ad hoc charitable donations or sponsorship, there was a diverse spectrum of activities, with many evidencing long-term benefits and perhaps what could be perceived controversially 
as more meaningful CSR engagement. Such projects such as this contribute to the local community and society by focusing on human development. For example, teaching skills to unemployed refugees so that they can earn a living in the long run and contribute to society.

In this case, the intention of the project was not to create any form of shared value other than personal satisfaction. This ethical perspective is similar for many of the SMEs, regardless of the way that they conceive CSR or the diverse activities they engage with. As can be concluded from above extracts, there are misalignments with the way that owner-managers relate to and make sense of the term CSR in comparison to their actual engagement.

This misconception is often as a consequence of context related factors such as the economic landscape or organisational characteristics of SMEs such as embeddedness. For example, some smaller businesses misconceive CSR as a concept that is more suitable for larger corporates with greater amounts of discretionary profit, as was seen in the quote by P21 above. P3 even suggested that the word 'corporate' actually holds negative connotations for some SMEs, as seen below:

\section{P3: “...the word corporate doesn't necessarily, you know the companies that I represent and work with in these scenarios are not individuals who would like to use the word corporate um, corporate is...tends to have um...the type of people we're dealing with it has a very negative connotation so it's not something we use". (p. 11, 300- 304)}

This conception of the CSR term as something only relevant to larger corporations is again shown in the extract below. It is considered as something pursued with growth or when at some minimum level of operation as a business, triggered by the expectations of stakeholders. The SME below excludes themselves from the CSR debate and expectation to deliver, implying a perceived lack of impact due to the scale of the SME. It seems fair to consider that SMEs do not recognise the impact of their business operations collectively on society. 
I: "And do you think the term corporate social responsibility is relevant in this case?"

P23: "No, I think that's important for larger organisations. But because we're so little, I think people appreciate. I think there's no expectation for me to deliver on anything like that for my clients is what I see. But, again, in line with growth, I think people expect it, when you're at a certain level, yes. But for now it's not really relevant". (p. 14, 377-380)

Further reasons for the conceptual nuances of CSR regards the national cultural values of SMEs in the UK and more specifically Welsh context. SMEs are characterised by the everyday fire fighting of tasks and a short-term focus towards business operations as demonstrated below:

P27: "A lot of the time you're firefighting and dealing with things as they come”. (p. 22, 606-607)

Fire fighting is an inherent part of SME operations, yet the short-term culture perceived below does not facilitate the adoption of responsible and sustainable business behaviour. P10 felt strongly about the inappropriateness of the short-term view of business, his views are presented in the extract below:

P10: "The number of firms that go out of business is...particularly in Britain I think is quite a lot. I feel we've got a completely different view over here to say Germany which, I mean I'm always full of admiration for the Germans having all these family businesses or privately owned businesses that last for generations. But we've got this view over here which I think is really sad that you build up...if somebody starts a business and say builds it up and then they sell it and they go off to a desert island or whatever, which I think is really sad”. (p. 11, 312-322)

This particular owner-manager further goes on to explain how a culture is developing of serial entrepreneurs with the view of starting up SMEs to develop and sell on. This promotes a get rich quick vision, not capitalising on the potential longterm success and contribution to society: 
P10: "I think it's a really bad British view of...why would they want to sell the business? Okay, everybody likes a decent lump of money but quite often the money you're going to get at the end will not reflect the earning capacity of the business really". (p. 11-12, 324327)

P10 describes this short term and profit driven culture as problematic in terms of facilitating responsible business:

P10: "I think this is a really bad British view of, it's a bit of a get rich quick thing and not having a social responsibility to be honest". (p. 13, 353-354)

P10 appears to be referring to larger family owned businesses in this case, but claims that SMEs should be started with a long-term view of sustainable business, with the culture in the UK not currently accommodating this. On the other hand, findings expanded on in Chapter Seven evidence that SMEs value and often rely on long-term relationships with stakeholders on an organisational level. The diverse and multiple influencing factors make apparent the complexity of the CSR concept and contribute to the inhibiting of conceptual solidarity.

Finally, for many SMEs the emphasis was just on survival of the business for the benefit of the individual. These individualistic values can be seen as both cultural and contextual organisational aspects that influence the perception of CSR for SMEs. They can also adversely affect CSR awareness by inhibiting the concept as one that needs to be presented to the SME, rather than actively sought. For example, P7 below explains as a start up in the initial stage of the business life cycle, the lack of time restricted their focus. They therefore would participate in CSR if stakeholders came forward and presented the opportunity, but otherwise the focus was on the business:

P7: "We just literally have no time, like at all to help people, like it's okay to help for a few hours and we do always say yes, I think when we get the opportunity to be socially responsible we take it but there's not really many opportunities that come our way it's not like we have an opportunity and we're like oh I don't have time for that it's like we don't have the opportunity presented to us, but maybe that's because we've just started out". (p. 9, 166-171) 
The above quote highlights a further misconception of CSR, whereby SMEs believe that it is something that should be requested by stakeholders and opportunities presented to the SME rather than proactively seeking ways to be responsible. This suggests a need for awareness of the scope of CSR for SMEs, the need for a sustainable and long-term perspective of the concept and perhaps exploration into the differing views of the concept at each stage of the life cycle for the SME.

Finally, despite the misalignment of understanding for CSR in SMEs, every business that was interviewed, regardless of the context, sector, stage of the business or any other contributing factors, participated in what would be considered socially responsible activities. It was the lack of unity on what the term constituted and certain contextual inhibitors that restricted awareness and the recognition of the contribution of SMEs to society as a collective.

A finding from the focus groups was that SMEs aware of the full concept of CSR saw it as a duty to disseminate knowledge to those not currently engaged or educated on the concept. The advocating of SME owner-managers as the greatest resource for fellow SMEs could be utilised as one way of addressing any misunderstandings, educating and pursuing conceptual solidarity of CSR for SMEs.

\section{4: Individual Conceptualisation of SME Responsibilities}

Having considered the cognitive awareness of owner-managers of the term and concept of CSR, the following section considers the individual conceptualisation of the broader responsibilities of SMEs. These responsibilities can be considered as the components that cumulatively amount to a social responsible SME and are structured around Carroll's (1979) CSR pyramid and Spence's (2016) SBSR pyramids. The first considered is that of the responsibility to survive and sustain an economically viable business.

\subsection{1: Survival, Economic Contributions and Sustainable Business}


When asked of their economic contribution as SME owner-managers, participants conceptualised the responsibility in terms of the duty to sustain their business and ensure it is economically viable. There were three main findings related to this responsibility. The first of these is that whilst some SMEs implied the desire to grow, many were content with a stable level of business to minimally ensure business survival and potentially the maintenance of the owner-manager's lifestyle. The second was the main economic contribution considered by SMEs, as the provision of employment and assistance in the maintenance and growth of other businesses. The collective importance of SME survival was acknowledged, particularly for localised regions and communities, but also as a contribution to the overall wellbeing of society.

The traditional conception of the economic responsibilities of a business would most commonly follow the tenets of the extract below:

\section{P2: "I mean our main responsibility is to earn money and that, that is the number one thing so we are focussed on more than anything". (p. $1,19-22)$}

However, this view of the responsibility to drive maximal profit was only made explicit by one participant, whom at a later stage continued to reveal an array of CSR engagement of which there were no tangible returns for the business. He also described a desire to interact more with the community in the future and provide a workplace whereby employees enjoy the process that results in earning money. This evidences a large degree of equivocation with regards to the key responsibilities driving the business.

The majority of owner-managers in the interviews conceptualised their economic responsibility in terms of survival or profit satisficing. Despite a number expressing a desire of growth, the emphasis was commonly on satisfying economic needs rather than purely maximising profit. Ulterior personal motives and elements of selfactualisation were often described as the aims of the SME. The example below evidences a unique perspective of the economic responsibility of the SME. This perception is morally grounded and derived from the personal values of the ownermanager: 
P29: "I'm probably the worst manager, but it's values that's passed down from my mum". (p. 3, 75-76)

She continues to explain by saying:

P29: "I've got my morals there that I know if something's not going to take me that amount of time. I know what other accountants charge and I know that they do rip people off, but I know, obviously, they've got the credentials and the experience and all this and they've worked hard to get to their level, but I still think, 'well, I can get by on that, our business can survive on that, I'll charge that'. So, it's more customer satisfaction based and yes, we're not money-makers. That's not what our aim is. It's more to create a workplace where we all enjoy working, but the customers have a good experience". (p. 3$4,82-90)$

The above extract is unique considering the context of the SME. It is unusual that an accounting SME would not consider themselves as 'money-makers'. Instead, their economic justifications are drawn from personal values and morals whereby they charge a price to 'get by' and 'survive'. The aim instead was more holistic to create a happy and fulfilling workplace and to provide a quality service. This might not normally be the case for professional service businesses such as accountants, however similar notions regarding profit are shown by P22:

P22: “I don't rate money that highly. I think there's more important things in life so I think it's important, it's more important for me and always has been, to enjoy my job". (p. 3, 82-84)

She later says:

P22: "We're not making a quick buck, you know, we're not here to con anybody or to get rich quick; it's sustainable, it's doing a good service at a good price”. (p. 3-4, 88-90)

The owner-manager explicitly claims that they do not rate money highly and are not trying to 'create a quick buck' or 'get rich quick'. Their priority instead was to build their SME on honesty to create a sustainable business and provide quality service at a fair price. Whilst it is acknowledged that there needed to be some kind of economic pursuit, this SME was not purely profit driven. Instead, they were 
additionally driven by personal morals, a strategic view towards sustainable business and the wellbeing of the owner-managers. These were all key notions underpinning the economic pillar of responsibility.

This conception was not uncommon during the interview findings. P9 further supported this view, attributing differing economic focus of SMEs to the stage of the business in terms of their life cycle. In this case, the start up phase was described and economic responsibility considered as simply breaking even:

\begin{abstract}
P9: "Obviously it's a new business so when we first opened the Marina we just wanted to break even so that wasn't, the profit margin wasn't a massive priority, but we just wanted to make sure that we didn't lose anybody as such, but it's all about gaining customer database, getting people to come back that was the main priority is treating the customers well and obviously treating the staff well so that they stay on". (p. 2-3, 57-61)
\end{abstract}

In summary one of the key responsibilities of SMEs was not perceived as profit maximisation, but profit satisficing, long-term survival, a working environment reflective of personal values and accommodation of the long-term retention of staff.

When asked of their economic contribution, owner-managers frequently referred to their role as employers, recognising their importance as stimulants of job creation and consequently catalysts of societal wellbeing. This was recognised as both the collective contribution to the wellbeing of society more generally, but also for the local and regional labour markets. One SME owner-manager explains this collective contribution to the economy below:

P22: "Yes, obviously in a general sense we employ people so we give people jobs, we pay taxes, collect VAT for the government so yes, certainly, yes, I think small businesses play a huge part in terms of helping the economy as a whole”. (p. 1, 5-7)

This was claimed again in terms of the role that SMEs play in creating jobs and educating citizens in order to contribute to their skills set so that they can enter the job market: 
P26: "I think that when you're a small business and looking after a team of twenty five we're helping with the economy that way...and helping both with jobs and education". (p. 1, 1-3)

The responsibility of SMEs to endeavour for long-term survival was recognised on an organisational level as the owner-manager below articulates. The implications that this perceived responsibility has on employment on a business level are recognised as complimentary for both business and society in general. The important role of maintaining employment within the SME and the contribution this has to their families is described in the extract below:

P10: "The fact we employ about 105 staff and naturally the older I become I think the more I realise that naturally the business, the success of the business is so important to keep that number of people employed. You know, the contribution it makes to their families too really”. (p. 1, 7-11)

Participants further expressed their economic responsibility as not only providing support towards solving unemployment more generally, but by forming an SME and custom made job for themselves. Particularly for the smaller SMEs such as micro size, as individuals their SME allowed a job that facilitates other commitments, rather that alternatively being forced to claim state benefits. The below is the response of a micro business owner-manager when asked of their economic contributions towards society:

P15: "I don't know if I have one, I pay tax, I pay national insurance so yeah I guess it does, well it gives me a job, the only other option with the kids would be to sit at home and claim benefits so at least I'm working”. (p. 1, 4-6)

P20 further supported this by suggesting that when assisting someone and getting them off benefits, the provision of employment is in fact a form of giving back to society and assisting in sustainable economic prosperity:

P20: "I am at a point now where I can give someone a job and pay you know, someone's wage and help them get off benefits. I suppose that is one way of giving back”. (p. 1, 7-9) 
The contribution of SMEs in terms of relieving the pressure from government was articulated in great detail by P27. This SME was founded on the notion of responsibility and was pushing for improvements in societal wellbeing by addressing unemployment issues and providing education programmes for the unemployed. Their contribution as an SME is detailed in the passage below:

\begin{abstract}
P27: "In terms of the benefit for the economy, it's pretty huge to be honest. For example, last year we delivered a large Department of Work and Pensions contract, these are long-term unemployed people that had been out of work for two and a half years plus. For that alone we helped 46\% of people into work, who came on the programme and our DWP target was only 20\%. So that alone, from people's disposable income from going from benefits into paid work was, I'd argue, is huge". (p. 3, 66-72)
\end{abstract}

The participant refers specifically to CSR as being incorporated into all programmes delivered by the SME, saying:

\footnotetext{
P27: "All of our programmes are geared towards upskilling and raising people's human capital for them to progress into sustainable paid employment”. (p. 3, 82-83)
}

Obviously this is the perspective of an SME delivering public sector contracts with the whole company founded on helping people and enriching the overall wellbeing of society. Yet, it still has a valuable contribution in terms of expressing in detail the massive economic contributions to the local economy and wider society that SMEs have. The importance of retaining money locally was frequently considered an important economic responsibility for SMEs as a contribution to their communities. An example of this importance is below:

P9: "We're not a massive brand, but we are keeping the money in [Location 1] so we try and use as much as we can local produce and I think all of our suppliers apart from one is in Wales". (p. 1, 9-11)

And again by P22 in reference to suppliers and keeping money in the local economy: 
P22: "We prefer to use local, again, that's part of the ethical thing. I do believe in kind of keeping the money in our local economies". ( $\mathrm{p}$. 12, 309-311)

As was already touched on by P27 above, a further theme of economic and societal responsibilities was the up skilling and contribution to assist the survival and growth of other SMEs. This theme was continued by P4 in the following extract, whereby his SME provides an online sales channel to help SMEs grow:

P4: "In terms of the economy I'm helping other small to mediumsized businesses to transact online, so I'm giving them this as a sales channel to help their business grow so obviously that's generating turnover and profit for them and from a social point of view, because of the structure of my company I offer a service whereby I can deliver competitive rates for the services that we offer, because my operation is based offshore". (p. 1, 9-14)

The nature of the SME business operations is perceived to contribute as one of their economic responsibilities. However, one of these economic contributions is based on the reduced rate of the service for other SMEs. Conversely, something to consider is that due to the offshoring of his business, it redacts from employing those in the UK and local to the actual business.

$\mathrm{P} 1$ additionally contributes to this theme via her understanding that surviving as a business and providing skilled work to the increasingly competitive job market contributes as one of her economic responsibilities and contributions:

\footnotetext{
P1: "I would say our responsibility is really to help other small businesses to grow, so we're a marketing agency, our number one goal is to...make another business survive, or help their message come across in the right way, but for me personally as a business owner it's about building a business, bringing more people in to work, um and giving people the jobs that they really deserve”. (p. 2, 50-54)
}

The above findings support the view that SMEs are characterised as profit satisficers, instead prioritising employment for themselves and others. They further conceptualise their economic responsibility in terms of stimulating the labour market, the assistance of survival in other SMEs and maintaining a sustainable and 
long-term business operating in alignment with their personal values. These all exemplify SMEs as having a large contribution towards economic development and societal wellbeing.

\subsection{2: Legal Compliance}

As a definition, CSR requires businesses to voluntarily engage socially beyond the minimum legal requirements. However, when participants were asked of their economic and social responsibilities, the adherence to laws was as a majority, absent from testimony. SMEs rarely commented on the compliance of laws as a contribution to their social responsibility, despite it being obvious that this is an essential business contribution for most. There were a number of examples when SMEs did refer to their legal compliance; for example, adherence to health and safety laws, environmental management systems and adopting the living wage beyond the national minimum. However, still the discussion was limited.

A further reference to legal compliance was made by P15, although this was of a different nature. As a design and manufacture SME, the non-compliance of copyright laws from competing SMEs was resulting in unfair competition and the inability to compete:

\section{P15: "A lot of these small businesses, well I say business, I don't even know if you can call them a proper business, but they always break the copyright laws. I find that there's so many things that are Trademark and you can almost guarantee that none of them actually have a license to sell it, but when you're buying the rights to use something and you're buying vectors, I find that really hard to compete with”. (p. 5-6, 140-147)}

The risk of breaching copyright laws was not seen as feasible for P15, yet many of her competitors were openly doing so in the informal economy, creating an unfair advantage. Businesses are expected to adhere to the law because it is the codification of what society views as acceptable or unacceptable behaviour. However, for SMEs, full compliance with laws is often unlikely due to a number of reasons such as knowledge gaps of SMEs, insufficient resources to fund outside specialists to ensure compliance or the perception that they will have an insignificant 
impact. These points may be contributing factors to the limited presence referring to legal or altogether extraction of legal adherence when conceptualising CSR.

On the other hand, the absence of legal compliance from conversations when ownermanagers were discussing their economic and social responsibilities could also be due to the societal context of the research. The UK is a developed country and compliance to laws is commonplace, with limited corruption and deviance, as often may be the case for developing countries. The compliance with laws may therefore have been overlooked as an obvious requirement for business survival and considered as part of the responsibility to be economically viable. This could also be viewed positively as SMEs are not merely viewing their social responsibilities as motivated by legal compliance, but instead as integrated in their business operations. In summary, for some SMEs the adherence to legal requirements is not considered as a fundamental priority or indeed the most fundamental ethical responsibility. The limited discussion of legal compliance as a responsibility could indeed require further exploration however; it is beyond the timeframe and focus of this research.

\subsection{3: Ethical Standards, Norms and Expectations}

When talking of the responsibility to be ethical, many participants spoke of doing what was right and fair towards those whom they have a responsibility to. This could be acknowledged as the traditional conception of ethical responsibilities with regards to CSR (Carroll, 1979). What became apparent was that owner-managers conception of ethical responsibilities went much further than this; they appeared to go above and beyond the expectations, to nurture relationships and care for stakeholders close to the business. This demonstrates a level of accountability towards stakeholders and empathy towards their wellbeing and happiness.

As was witnessed in the focus groups, SMEs are characterised as being interconnected, embedded and reliant on networks and stakeholder relationships. Stakeholder networks are complex and context specific, largely dependent on each SME. However, one uniting factor was the close association owner-managers felt towards them. As P18 explains, networks closely tied to the SME are often relied on 
in order to maintain the business services, with close friendships transcending boundaries:

\section{P18: "I could work on my own but not what I do now, I need my employees and a wider network to deliver what I'm delivering, so close friendships come actually across boundaries within the network". (p. 4-5, 120-123)}

This reliance on close relationships and networks is a unique characteristic of SMEs. Owner-managers often work closely with stakeholders in order to nurture synergistic relationships. When asked of the main responsibilities of their business, P6 as a social enterprise described the priority of caring for individuals, in this case specifically employees of the business:

\section{P6: “Um, I think I'd say caring for individuals, so looking out for everyone and focusing on each customer and taking each customer one at a time, is really important for us". (p. 3, 71-73)}

Caring for stakeholders of the business on this level would not commonly be considered an expectation of the business or moral right from societal members. This level of care from SMEs towards stakeholders goes above the traditional conception and redefines the ethical responsibility as expected by society. The codification of this redefined ethical responsibility would not be possible due to the involvement of intrinsic, social bonds, therefore this can only be considered as a redrawn conception of the current ethical responsibility of business.

One participant even went further to say that his personal survival, happiness and future is linked to those of his employees. Ensuring the happiness and flourishing of his employees shows an extension of just meeting the need to be ethical towards them. This is interesting considering that this SME referred to their main responsibility as being the pursuit of economic success above all else. Yet, the extract below implies an element of care:

I: "Which stakeholders would you consider to be your main priority?” 
P2: "Staff".

I: "Staff, okay um and on what basis do you prioritise them?"

P2: "On what basis that um my survival is relying on their survival and how much they flourish and that I've always believed that we kind of work to get, you know it's like all of ours powers and futures are linked and therefore their happiness is my happiness and that kind of thing”. (p. 4. 89-96)

Although, this level of care could be due to the sector of the SME, as a recruitment company, the success of the business is largely based on the competencies and efficiency of employees. The happiness of staff therefore is set to translate into personal success for the owner-manager, obscuring the true motives of the pursuit of their happiness.

On the other hand, P21 explains that, rather than a need for individuals to 'fit the job', often a more personal approach towards the management of stakeholders is adopted by SMEs. This relational approach is particularly important as a responsibility for SMEs and could be due to and in part counteracting the lack of specialist human resource personnel within the business:

P21: "Fairness, generosity, respect for the whole person. So for example, we've always accommodated all our staff around their needs, their personal needs, more than 'this is the job, you need to fit into the job'”. (p. 2, 40-42)

Common similes are used likening relationships with key stakeholders to that of family, as will be discussed in more detail in Chapter Seven. In this instance the input from stakeholders drives a more succinct approach towards being ethical and suggests more than just fairness. This merging into the private sphere therefore brings about more of an affective relationship and what could be seen as heightened feeling of responsibility towards them, more than just what is fair and expected by stakeholders. P1 describes her employees as her children and a deep level of empathy for their needs and responsible management in the two extracts below:

P1: "We literally try to do anything that we possibly can for our staff”. (p. 7, 182-183) 
P1: "We do care about them, they're literally like my children you know”. (p. 7, 190-191)

Many SMEs implied that their ethical responsibilities go past just being respectful of the needs of their stakeholders. Socially responsible activities such as community engagement events were held purely to build relationships and networks for the community.

P6: "...because our focus is building community in relationships, we do um like meals for regulars now so yeah all our regulars once a month we do a free meal in the evenings". (p. 1, 5-7)

In fact, P7 purports that actually, relationship building within the community is more important to them than taking their money:

P7: "The other day we had a shop opposite us and they needed like some labels sewn in as a favour and we went away and did it and they were like 'well how much do you want for it?' and we were like 'oh no it's fine' like and that was more important to us to like build up a relationship with our neighbours than to take their money”. (p. 4, 100-103)

Although P27 has the view that by building such relationships that go beyond just meeting the minimal needs and expectations of their stakeholders, it does actually result in better business with tangible outcomes, particularly when trying to deal with larger organisations:

P27: "If you work at those relationships with stakeholders, I think it does definitely ... it does result in better business. And, particularly if you're a small provider, and you want to have sub-contracts with these larger organisations to deliver part of their contract on their behalf, they always take a slice off, like a management fee, but I think having those personal relationships does generate extra business". (p. 15, 429-434)

Finally, P14 goes as far as saying that actually, being nice to people does get you places. They highlight that the caring element and niceness towards people additional to expectations is a unique, distinguishing feature of small businesses that aids their survival in comparison to large corporates. 
P14: "I do think that being a nice person does get you places though, well I hope it does. I think that, that maybe is what gets you by as a small business in comparison to a corporate". (p. 3, 82-84)

To conclude this section of findings, the SME conceptualisation of ethical responsibility goes beyond adhering to the activities expected or prohibited by societal actors. The highly personalised and interconnected nature of business for SMEs appears to intensify the ethical responsibility to redefine it as one based on more than just fairness and rather enhanced relations with stakeholders moving towards more affective levels of involvement. Compassion and care was shown towards stakeholders and values placed on the nurturing of relationships, suggesting that the realities of actors are connected.

\subsection{4: Philanthropic Responsibilities}

Philanthropy is traditionally considered as those activities contributing to society that go above and beyond expectations. One participant perceived philanthropy as being the responsibility towards those that they did not have a contractual relationship with. As the quote below implies, the ethical responsibilities of his SME relate to those whom he has some kind of existing personal or business relationship:

P18: "Ethical, I tend to keep the ethical responsibilities where you have a contractual responsibility and philanthropy where you don 't". (p. 10, 294-296)

He explains further his conception of philanthropy:

P18: "Philanthropic activities do go last, not because it's not important but you can't be philanthropic with someone else's money you know um as much as it's a personal driver for me um I can't do it, the people to whom I have a contractual responsibility, like the wife because we exchanged marriage vows, the kids because we brought them into this world and you know employees and clients and collaborators um you know they have to come above people to whom you don't have a formal responsibility, you only have a moral and an ethical one”. (p. 5, 135-141)

This perception of contractual relationships is interesting, particularly considering the opinion of having a contractual relationship with his wife and children as key 
stakeholders of the business. He viewed philanthropy as being a large personal driver, but one that comes last in terms of responsibilities for the SME due to the perception that you cannot be philanthropic with someone else's money. This hints an essence of Friedman's (1970) perception of ethical responsibilities of a businessman being purely the pursuit of profit. However, the inclusion of the ownermanagers family as being a contractual responsibility of the business deploys from this perception to an extent. Furthermore, he still refers to the moral and ethical responsibility to those that the SME does not have a contractual relationship.

Surprisingly, religion was not featured as a driver of philanthropic activities, despite it being traditionally rooted in the culture of this country via early philanthropists of the industrial revolution. On one occasion the owner-manager of a social enterprise did describe the morals of the management team and spirituality of the SME as coming from a religious spectrum as is seen below:

P6: "On director level, there is definitely an element of like spirituality in the shop and our morals are totally like coming from Jesus". (p. 12, 359-360)

They proceed to explain how their SME is built on the foundations of philanthropy and their religious obligations, presenting a unique servant hearted nature of working in order to give back to charity:

P6: "There is definitely this like servant hearted nature in place that you're working to give our money back for these sorts of charities". (p. 4, 118-120)

However, as is mentioned this was a social enterprise and the common conception of philanthropic responsibility in general from the interviews was not founded on religion. This could be due to numerous reasons such as macro level factors including changing societal factors and diverse demographics, political structures, the economic system and culture.

More commonly philanthropy was a by-product of personal experience, interests or significant events in the lives of owner-managers, or someone close to them. As P10 
explains, they do not have a process in terms of philanthropic giving; rather it was more down to personal interest:

P10: “There's not that much of a process I'd say. It's more, almost more down to personal interest and that sort of thing". (p. 23, 638639)

P8 further supported this when claiming that naturally individual actors will show partiality towards those causes that mean something to them:

P8: "For me personally it's because I've been affected by it. That would be the biggest reason why I get involved in it”. (p. 20, 511513)

Again this is not to say that other causes will be ruled out, but these defining moments in the lives of owner-managers will influence their support of particular causes or charities. For example, many participants described the drivers of philanthropy being due to significant events in their lives that have effected themselves or those close to them, with family illness being referred to in the extract below:

P1: "We did the you know, wear a hat for brain tumours and stuff like that and my mum had a brain tumour around 8 years ago, she's fine now but she had to have it removed so that was a massive pull for me to give to that". (p. 15, 410-412)

As can be seen this individual influence on philanthropic efforts was also partnered with the influence of key stakeholders within close proximity of the owner-manager or SME. For example, one of the most consistent influencers of CSR was the employee, as seen below:

P1: "At the end of every month that money from the tuck shop goes to a chosen charity by each team member, so the team members can pick where they want that money to go to". (p. 2, 32-33)

The same company further influenced their key customers and rather than sending gifts at Christmas, they donated money to a charity of the customers choice as is displayed in the extract below: 
P1: "People get to pick where they want the money to, well it's not their money, well it is because we would have bought them a gift otherwise, um, um but what we've also been able to do is because of our links with a lot of our customers we've been able to get a couple of customers on board, so they're also donating the money that they would have given their clients, so it's really good". (p. 1, 14-18)

It is important to recognise that philanthropic efforts from SMEs were different in character to large corporations. There is still a large emphasis on charitable giving and sponsorship, often with significant events in the lives of individuals close to the SME driving such philanthropy. However, a preferred form was often the donation of time and skill, with engagement in activities that are parallel with their business competencies rather than seeking more generic categories of CSR or those personal to the owner-managers as an individual as seen below:

\section{P3: "Um....yeah in some respects if I look at it this way I'm a volunteer for mountain rescue so being a small business owner it allows me the freedom to be that volunteer I couldn't really do it if I was working for my last company um, so it gives me the freedom of giving my time, which is think is giving to society". (p. 1, 11-14)}

To conclude, in this particular instance owner-managers make sense of their philanthropic responsibilities via a spectrum of CSR activities. These are most commonly charitable giving and sponsorship and the donation of their time and skills as most favourable. The first conception of philanthropic responsibilities was directed towards those without a contractual relationship with the business. What was found is that personal experiences and the influence of key stakeholders within proximity to the SME are what seemingly shape philanthropic practises within this context, despite the tradition of religion being a dominant driver in the past. It was evident in the findings that the conceptualisation of philanthropic responsibilities stemmed most commonly from individual values and experiences, along with relationships that transcend both the personal and business spheres.

\section{5: Motivations and Drivers of CSR}

\subsection{1: Intrinsic Motivations and Moral Obligations}


Continuing from owner-managers conceptualisations of business responsibilities, the following section is of importance to display findings that evident key motivations and drivers for CSR engagement. For example, is CSR categorised as a strategic activity motivated by economic rationality and the orientation towards profit making? Is it a pragmatic response to increasing stakeholder demands and wider contextual pressures? Or is CSR a morally grounded phenomenon, seeking no return with an altruistic approach? The motivations for SMEs to engage with socially responsible practises evidenced nuances of ethical, relational and on more limited occasions what could be considered as instrumental drivers. There was a common consensus amongst SME owner-managers that CSR is not something that should be done for profit implications, as expressed by the two participants below:

P13: “You shouldn't be doing it for more profit”. (p. 17, 433)

P12: "I don't do it so I can get money and get people, I do it because I like to give a little bit back". (p. 12, 315-317)

This is demonstrated by the act of P12, whereby he does not claim his CSR efforts back through tax, implying a genuine altruistic motive, not economically driven:

\section{P12: "Yeah you can claim that back through tax. I don't, I don't claim it back through tax. I know you can claim it all back anyway". (p. 15, 389-390)}

As identified in the entirety of Chapter Six and in the conceptualisation of philanthropy, the personal values of owner-managers and significant life events directly influence the inception and engagement with CSR. Significant events in the lives of the owner-managers will motivate them to support particular causes or charities as a form of CSR, as seen in the quote below:

I: "How do you make that decision as to which activities you support?"

P8: "Just on what it means to me personally and that's not to say that if someone comes in from the Diabetes Association that I'll just rule it out but you do, don't you? You naturally go for things which mean something to you". (p. 18, 448-450) 
The personal interests of the owner-manager are also likely to stimulate intrinsic motivation to engage with socially responsible activities. This was the case for P21 below:

\section{I: "How did you get into that?"}

P21: "Yes that was a personal interest and I just thought...I just felt really...I've spoken at two of these sort of anti racism rallies, I got invited to talk because I'd got an article in the paper because we'd made some banners when there was the kind of welcome the refugees thing and we'd made some banners and we got in the papers. Then I got invited to speak at the rally and then I got invited to speak again and it's just something I feel really passionate about". (p. 20, 540545)

Alongside personal interests, one of the prime CSR motivations for many of the participant SMEs arose from a sense of a moral obligation to give back. This was specifically as an appreciation to key stakeholders or local communities that help accommodate the survival of the SME or directed towards those that need assistance. The notion of ethics and doing what was perceived as 'right' in society was referred to as a morally induced motive for P2 and not economically rational:

P2: 'It's always the right thing, what's fair and what's right, that's always again the bit that we fall back on, I've got a business partner and we go with what's the right thing to do and not, not what's the financially best thing to do, what's the right thing to do". (p. 5, 130132)

This sense of duty stemmed from an appreciation of their current established position within the community. This acts as a moral stimulant that drives CSR. However, it is reasonable to assume that there is some notional level of acceptable profit or success that is pursued before this moral stimulant is activated. This is evident from the owner-manager below who reveals a sense of duty, but predicates it on the fact that they are in a position where it is possible to give back, we can assume in this case that this refers to an economically stable position:

P15: "Yeah a duty, I just think that everybody has got a responsibility really but because I'm in a position where I can do it I feel like I should”. (p. 8, 220-221) 
This view is replicated by P9 that the position of the SME is a premise to the sense of duty towards their community. Although, in the extract below it is not clear if this is the situation of the owner-manager or the SME as a whole, as often occurred in the interviews as synonymous, as is exemplified in the below:

\section{P9: "I also say that I'm in a position where I can do that, so a lot of people would love to but they're not in a position to be able to, so I shouldn't really take that for granted, I should be there because I am part of the community". (p. 13, 372-375)}

Whilst the above motive is seemingly proactive behaviour from the SME, a further driver related to a sense of duty towards the community on a reactive basis, is the provision of assistance to those that are in need. Many in the interviews referred to the conception of CSR as the donation of money. The below example refers to their motive specifically being the contribution of money to such recipients:

P25: “...contributing to people who need it, people who need the money”. (p. 14, 357-358)

Empathy and appreciation towards the local community commonly induced an impulse to engage in responsible behaviours. However, the communication of such efforts was not seen as necessary and often no specific reciprocation was expected. Yet as is seen in the extract below, the participant did not require 'too much' publicity back from their community engagement. This implies that there are some mutual benefits on an organisational level in terms of public relations:

\footnotetext{
P10: "I think it's a good thing to do, it's good to support the community which is feeding us really and you know, it's...we don't really need to be seen ...you know, I don't...we don't really require too much publicity back in that respect, it's not a sort of tit for tat really, it's just one of those things". (p. 26, 715-719)
}

Another owner-manager of a recruitment company again expresses their motive as an appreciation of the local environment in enabling their success. This is interesting as the business operations of this SME varied UK wide, yet their sense of duty was towards the local community that the SME was situated and sourced their staff from. 
P2: "I think that as a successful business kind of part of your success is due to your environment and where you are so it seems that the least that you can do is to give back”. (p. 9, 222-224)

Parallel to the sense of duty experienced by participants was the intrinsic feelings that are elicited from giving back to the community. The feeling of doing something worthwhile for the community and society at large was considered enough to drive CSR engagement for this SME:

P14: "I guess it's sort of giving something back to the community I guess as a feel good factor, so maybe you'll feel like you're doing something worthwhile really and things like that". (p. 5, 138-140)

Such intrinsic emotional rewards were again expressed by P2 whereby he referred to the satisfaction of collaborative team efforts towards CSR. This confirms that CSR is not just an individual effort from the owner-manager, but often with contributions and collaborations with other close stakeholders:

P2: "I think that there is a sense of satisfaction when you do it as a community and that you've done good, you know the collective group of people and you've just got to go 'we've just done something that we should be proud of' so I think that's good and everyone feels good about themselves then". (p. 10, 261-264)

Despite the dominant sense of duty towards the community specifically in these examples, there is still more that can be done to give back and raise awareness of socially responsible business practises. An SME in the accountancy profession raises the opinion that all businesses should give back to the community in some way, wishing for a world where everybody gives something back:

P29: "I think all businesses should give back to the community in some way”. (p. 21, 597-598)

P29: "I would like to see a world where everybody does give something back”. (p. 21, 599-600)

Finally, P18 an owner-manager of an engineering SME, talks of the satisfaction of applying his skills to the assistance of the community as a reactive form of CSR. He 
was presented with the opportunity to apply his business skills in the form of CSR to the benefit of the small village that he resides in. He describes it as 'my little Welsh village' identifying his proximity and identification with the physically located area;

\section{P18: "My little Welsh village has welcomed us no end, and I have had probably an unusual opportunity to apply my business skills to their benefit, well it was a planning application”. (p. 8, 211-213)}

This effort resulted in not only a solar farm being built rather than a sewage works, contributing to environmental sustainability, but also utilised intangible competencies of the SME, in this case the skills of the owner-manager. This CSR effort was localised to his home community, despite the SME business operations taking place worldwide. The motivations expressed by the owner-manager was the intrinsic feeling of satisfaction and pride of winning a more sustainable alternative for his community:

P18: "It was actually quite satisfying to bring my actual day to day skill into the local community, so it was good for them and it actually gave me a buzz, it was good and the fact that there is now a solar farm not a sewage works, well we won". (p. 9, 246-249)

Motives towards the development of the geographical locality of either the SME or owner-manager were regularly identified during the interviews. P5, a tourism and leisure SME describes the motive for CSR as being the desire to see the local area great:

\section{P5: "I want to see this area great, so you know I don't mind putting} my time in". (p. 12, 330-331)

On a broader level compared to the intrinsic drivers discussed above, the motivation to contribute to wider societal wellbeing was also referred to as being a driver for CSR engagement. P27 underpins this altruistic motive as providing support for the general welfare and human development of individuals and the contribution that this has to society. CSR can commonly be seen in the form of human development, with SMEs often identified as welfare maximisers as demonstrated below: 
P27: "What motivates me? I know it sounds really cheesy, what gets me up in the morning is knowing that we're helping vulnerable people to turn their lives around, to better themselves". (p. 20, 569-571)

P29 again refers to the motive of helping people with their SME with regards to the sustainable and long-term wellbeing of both their family and society in general. This is interesting considering the SME is in the accountancy profession. However, the importance of maintaining a sustainable business and providing a future for their family, as a family run business, was a more important motive than profit maximisation:

P29 'It's a valuable service. So, it's not about the money. It's about helping people, for me and my family as well. Like, having something that we can all do together and that's an investment for our futures". (p. 22, 622-624)

Furthermore, the moral reasoning motivating $\mathrm{P} 1$ to act responsibly came from a more personal and long-term perspective. She expressed a feeling of moral duty to contribute to societal wellbeing, her justification being 'because we should'. However, her desire to contribute was also driven by the impact this could have on her potential children, as future dependents of her and the SME on a psychologically proximate level:

P1: "It's because we should that's the first one, but also when I have children I want them to be able to experience life as I've had it so for things not to be disrupted and ruined because of our laziness". (p. 16, 450-452)

In terms of drivers, one participant with a food service SME inferred environmental protection as a moral stimulant for being responsible. Again, the contribution towards the long-term sustainability of the environment was prioritised over the cost implications. This infers that CSR on this occasion was not driven by profit motives: 
P6: "This week we found a fully compostable take away cup, so we're going to go fully compostable with all our take away cups but it will cost more, so we're not doing it for any strategic reasons, it's just a case of well actually we should probably be doing this for the sake of the environment". (p. 15, 422-425)

These drivers collectively stem from the desire to contribute to the broader preservation of long term economic, societal and environmental sustainability. This desire stimulates such ethical behaviour. The impact that this potentially has for owner-managers personally and for those closely associated to them, strengthened the intrinsic motivation to contribute with this long term vision.

To summarise, key intrinsic motivations stem from the personal interests of the owner-manager and causes close to them. Moral obligations that also drive CSR engagement are often derived from a sense of duty towards physically proximate communities or directed towards those who are in need of assistance of some form. Motivations for CSR in SMEs can therefore be considered as often personalised, context specific and founded on the notion of altruism. Furthermore, individuals were motivated by the desire to contribute to broader societal wellbeing, in part to assist where help is needed, but also to assist in long term economic, societal and environmental sustainability due to the anticipatory effect that these could have on society and future dependents of the owner-manager.

\subsection{2: Organisational Motives and Relational Drivers}

The organisational level is addressed fully in Chapter Seven, however a common motive for SMEs was being responsive to the increasing demands from stakeholders and the relational benefits that come from such engagement, despite an active defiance against the use of CSR for instrumental, profit related purposes. These benefits include the nurturing of relationships, increased exposure or enhanced reputation and trust towards the SME as examples. These relational drivers were occasionally expressed openly as the key stimulant for CSR engagement in the pursuit of an economically sustainable business. However, most SMEs referred to them as welcome benefits, but not actively sought or expected. 
The perspective from P10, a wholesale and retail trade SME, was that CSR should be executed without expectation or strings attached. However, again he expresses that his SME does not try to accrue 'massive publicity', this implies again that there is some kind of outcome from their engagement:

\section{P10: "We're not trying to get massive publicity from these things. If we get a little bit fine, if we get nothing, okay. If it's a good cause ... you know, if the cause is very good then you should give it, you know, without any strings attached really I think". (p. 26, 723-726)}

P22 further values the perception that business is a social domain. However, despite portraying intrinsic moral drivers in the first instance, this is done on the condition that it does no harm to the business. This confirms an already axiomatic assumption that SMEs will pursue profit to some kind of degree, with implications that there is some kind of trade off for CSR in terms of resources. On this occasion, the economic sustainability of the SME is prioritised before that of the social. However, P22 makes the point that the outcomes of CSR engagement that positively assist the SME are a bonus and not expected, therefore their motives are not instrumental in nature:

\section{P22: "It's not really what I want to get back from it but it is about well, you get a feel good factor which is good in itself in a way but it's yes, it's just what makes me feel comfortable that I'm giving something back as you say and doing it without hurting the business and if it helps the business I think that's a bonus, it's not the be all and end all but that's a bonus". (p. 30, 825-829)}

Interestingly, P26 refers to the changing motivations of the SME with regards to the stage in the life cycle of the business. This suggests that motives and drivers are dynamic and change as the business does. At the early stages of the life cycle, the main driver for CSR was the potential for publicity. Once the SME progressed from the start up phase this shifted to CSR engagement driven by the personal interests of the owner-manager and those causes close to them: 
P26: "I think in the early stages I would have done it for publicity and just to um, put our name out there, but now it's a bit of both, I do it even if I don't get anything out of it, I do it because I want to support it or ... because I want to support a friend, or I want to support the charity or whatever, so it's both, a two way thing really". (p. 4, 101-104)

A further point of interest is the reciprocal nature of CSR for SMEs. For example, when the recipients of the SMEs CSR efforts are situated within close proximity, this is likely to have an impact of the business whether intended or not. P9 talks of benefits stemming from CSR efforts with regards to the increased use of their facilities as a beneficial tangible outcome:

P9: "I would say obviously if you are doing stuff in the community a lot of people recognise that, they think that's a good thing and then they're more likely to come and use your facilities in a positive way as well". (p. 13, 370-372)

P7 talks of similar ideas with regards to the reciprocal benefits of CSR, she refers to the reciprocation in the form of intangible benefits such as word of mouth:

P7: "I don't think we expect anything back from it but we just think it does have an effect in terms of our business, like the more you help, the better people will view your business, the more good stuff, the more good things people say about your business". (p. 6, 161-163)

She further presents the view that the more positive 'stuff' the SME can generate, the better the business is going to do:

P7: "The more good positive stuff you can generate for your business the better that your business is going to do”. (p. 6, 164-165)

Reputation is another intangible outcome that can be procured via behaving responsibly as a business. As P24 explains, it is particularly important for SMEs within close proximity to their community. This sometimes results in CSR becoming more of a proactive measure and risk adverse activity. Not only does this avoid negative word of mouth spreading and ensure the maintenance of reputation, but also can assists in the strengthening of stakeholder relationships as reciprocal benefits: 


\section{I: "What is it that motivates you to engage with CSR?"}

P24: "Reputation I would say, if we help others then we look good to other people outside then, like because it's always, with the nursery reputation it is a big thing so if you get one bad thing said about you it does spread, especially in a small town like this". (p. 4, 104-107)

Finally, one participant spoke of relational drivers with regards to the donation of time and expertise. This is a resource-based response to CSR and contingent on the expertise and resources that the SME possesses. In this particular case the ownermanager spoke of the donation of time in order to network with students or the public in the form of CSR. A form of open innovation was drawn upon with the expectation that there might be outcomes or ideas induced that might potentially add value to the SME. However, this was seen as a risk on the chance that there are no beneficial outcomes for the business. On this occasion the CSR motives could be considered strategic:

P22: "I'm giving time free even though it's not a charitable cause but again, I'm thinking well, it could be, it could further my aims in terms of just networking and speaking to people, maybe interacting with students or members of the public who have good ideas that we could help, we could be involved with somehow. So, yes, there's some giving back potentially from that, it's still a risk, I'm still giving my time free of charge on the chance something might come about". ( $\mathrm{p}$. 26, 721-727)

Similarly, P3 explains that often there is a commercial gain to be had by attending events as a form of CSR. This is purely via the development of brand awareness and the building of relationships, despite not having direct tangible outcomes:

P3: "I think in some respects donating my time to the pursuit, there is a commercial gain to be had, it's not a specific recruitment event they don't go to them to find a job but it's about brand awareness and creating...it's building a relationship which can promote your business". (p. 9, 258-261)

That being said, P28 suggests that cumulatively, there is a tendency to give more than you get back, regardless of whether there are intangible or tangible benefits: 
I: "Have you seen any tangible benefits from doing those activities? So you said that people were coming in or? "

P28: "Um... a little bit, but I think you actually, you, you tend to give more than you actually get back from it, but like I said it swings in roundabouts really doesn't it'. (p. 7, 176-179)

In summary, organisational motives and relational drivers of CSR were commonplace in the interview findings. This is on one part as a response to increasing demands of stakeholders, but also for the relational benefits that facilitate survival and prosperity of the SME. Relational drivers were considered both as a key motivator for CSR engagement, or often as a beneficial outcome that was not actively sought but positively assists the SME. Owner-managers described this assistance as being a natural reciprocation resulting in both tangible and intangible benefits such as increased business or enhanced word of mouth and reputation. Such motivations could therefore be considered as a proactive or risk adverse activity on occasions. Finally, there was consideration that drivers for CSR are dynamic and change dependent on the current situation of the SME.

\subsection{3: Instrumental Drivers and Tangible Outcomes}

It was not often that participants described instrumental drivers as the key stimulant for CSR engagement. In the previous two approaches to CSR, there was common consensus that it should not be something done to impact the bottom line positively, although, this was on the pretence that it did 'no harm' to the business. Furthermore, it is reasonable to accept some kind of minimum amount of profit that would be pursued predicating engagement, with the discourse of participants implying that there are often beneficial outcomes for their SME, whether sought or not. Despite this, drivers associated with purely the pursuit of short-term profit were less common.

Not one of the owner-managers explicitly considered the pursuit of short-term profit as the sole driver for CSR engagement. However, P17 explains below that as an outcome from charitable fundraising events, she would have a large increase in sales and consequently short-term profit. 
P17: "Well I would sell from them as well, you know I'd take thousands of pounds that week after a fashion show um, so yes I would sell from them but I'd also raise money for charity from them so it was a win-win". (p. 5, 128-130)

This did not appear to be solely an active pursuit of short-term profit, but a positive outcome of mutually beneficial CSR engagement for both the SME and the charity.

On one other occasion, the positive cost implications of taking on apprentices was the motivating factor to engage with this form of CSR. The driver being that the business did not have to pay the apprentice, despite them being included in staffing ratios. This consequently saved money for the SME and therefore had short-term profit implications, but could also be considered a strategic move for the SME.

\begin{abstract}
P24: "With a nursery you get like staff ratios so you have to have a certain amount of staff to a certain amount of children well, when a student is with us for longer than ten weeks they can be counted in the staffing ratios, so...sometimes if you get a long term student that could be with you for a year they can drop into your numbers so you would, wouldn't have to pay someone to work, whereas, so really it saves us money then... and you're helping them get their qualification”. (p. 6, 143-148)
\end{abstract}

A further case whereby instrumental motivators were indicated was due to certain CSR initiatives being required for tenders. The possibility of winning contracts and the profit implications of this for the SME can be considered as the main driver for such engagement. This is evident in the extract below:

P27: “We've just applied for the Welsh government quality mark in Youth Work, so again we're being assessed for the bronze award, so a couple of assessors will come out and look at how young people's views are taken into account when you're delivering the programmes, how you act on their feedback".

I: "And do you find that helps with getting your tenders?"

P27: "Absolutely. If I'm honest, that's probably the main reason why those are probably so important”. (p. 22, 619-626)

Finally, one last driver for the SME clients of an owner-manager was the potential tax savings. Despite being driven by the cost reductions, the SME owner-manager 
defends her clients suggesting that despite the underlying motivations not being in line with altruism, it is not morally incorrect and the money is being redirected in the form of philanthropy to charitable causes rather than going into the hands of the government:

\section{P20: "I have got like clients that come to me when they are on a tax break and they come to me because they need a marketing plan to totally smash it and I think well if the government made it a little bit easier then they would be more morally you know... well would you rather give to charity or give it to the government?" (p. 22, 614-618)}

In summary, there were limited occasions whereby instrumental drivers were described for engagement with CSR. SMEs did indicate instances whereby increased short-term profit was an outcome of charitable events, CSR initiatives are driven by the reduced staff cost, as a requirement for competiveness in tenders and finally as way of reducing tax bills. That being said, it is difficult to distinguish those motivations purely for short-term profit and those that are strategic in order to ensure the long-term economic viability of the SME.

\section{6: Chapter Conclusion}

It is difficult to distinguish the true implicit motivations for individuals CSR engagement, results of interviews are at risk of being tarnished by social desirability bias, yet the results above describe in detail what was evident from the interviews. To conclude, having displayed the findings above, it is evident that the drivers for CSR are multifaceted and complex. There was often ambiguity in owner-managers attempts to explain why they engage with CSR. However, they most commonly referred to CSR as altruistic, being rooted by morals, with no expected outcomes. Evidence also referred to CSR being an activity driven by the potential organisational and relational outcomes or as a proactive defence mechanism. Changing stakeholder expectations are changing the way that CSR is approached, often being a proactive activity for the SME in order to adhere to these expectations, as well as sometimes being reactive to the demands of those stakeholders of importance. This is partly a response to increasing demands, but also for the 
relational benefits that are linked to the informal characteristic of SMEs that facilitates survival and prosperity of the SME.

What was also interesting was the sense of belonging and social identification to the local community that provokes CSR engagement. There were strong implications that proximity to stakeholders is a catalyst for the SME approach to CSR. Social identification and an appreciation towards society was also evident, gratitude for providing the adequate conditions with which the SME can be successful acted as a moral driver providing emotional credit that stimulated CSR as a response. Similarly, a driver for CSR engagement was to assist those within society that are in need of resources or expertise to strategically aid in the long run preservation of society.

There was only one occasion whereby CSR engagement was seen as a risk if no positive, short-term outcomes occurred. CSR was not actively set in opposition or antithetical to what is seen as positive for business. In fact, owner-managers saw many beneficial outcomes additional to what was expected. There were limited occasions whereby instrumental, short-term outcomes were made explicit as the key drivers for CSR. SMEs did indicate instances whereby increased short-term profit was an outcome of CSR initiatives, or they were driven by reduced costs or as a formality to be competitive to tender. That being said, it is difficult to distinguish those motivations for profit and those strategic to ensure the long-term economic viability of the SME. Whether the pursuit of short-term profit makes the act any less responsible is a big question for debate. What seems obvious is that CSR for SMEs is most regularly altruistic and morally grounded or as a proactive tool for stakeholder relations, not necessarily for instrumental purposes. Drivers for CSR should be considered as personalised, context specific and dynamic, dependent on the current situation of the SME and their networks of stakeholders. These will be discussed in further detail in the following chapter. 


\section{Chapter Seven: Interview Findings (Meso - Organisational level findings)}

\section{1: Introduction}

The previous chapter detailed the implicit views, personal values and conceptualisations of owner-managers that influence socially responsible activities in SMEs. However, human actors are inextricably embedded in their context. The organisational level of analysis covered in this chapter is concerned with this context of SMEs in terms of key stakeholders and the scope of SME's social engagement as influenced by webs of relations. The following findings are structured according to the themes outlined in Table 7.1. These have been derived inductively from the interview analysis and informed by relevant literature. This is in order to address the appropriateness of Spence's (2016) redrawn stakeholder theory in line with the ethic of care and moral proximity.

These characterisations are in terms of family, friendships and long-term commitment, the merging of personal and business spheres, community embeddedness, networks, collaborations and the dynamic nature of SMEs. Relational structures such as these are founded on elements of trust, reputation, respect and loyalty with stakeholders, but have also been identified in this thesis as being mobilised or intensified by the actor's location with regards to different forms of proximity. Research questions on this level attempt to inquire into the nature of such relations and how they may influence or be influenced by the inception and engagement of CSR initiatives. 
Table 7.1: Interviews: Summary of Super-Ordinate Themes for Organisational Level

\begin{tabular}{|c|c|}
\hline \multicolumn{2}{|c|}{ Organisational Level } \\
\hline Super-Ordinate Themes & Sub-Themes \\
\hline $\begin{array}{l}\text { Characterisation of Stakeholder } \\
\text { Relationships }\end{array}$ & $\begin{array}{l}\text { - Family, friendship and long- } \\
\text { term commitment } \\
\text { - Inextricable personal and } \\
\text { business connections } \\
\text { - Community embeddedness, } \\
\text { reputation and word of mouth } \\
\text { - Networks and collaborations } \\
\text { - The dynamic nature of SMEs } \\
\text { - Stakeholder relationships and } \\
\text { the balance of power }\end{array}$ \\
\hline
\end{tabular}

\section{2: Characterisation of Stakeholder Relationships}

\subsection{1: Family, Friendship and Long-Term Commitment}

This research found that SMEs in the UK are inextricably embedded in webs of relations within their context. Participants often described stakeholder relationships on an affective level, with connotations towards friendship and family. Despite some SMEs being family run, the contributions below constituted a broad array of SME forms. These descriptions most significantly imply a high level of commitment to others and a feeling of closeness towards stakeholders as dependents of the business. This was most commonly towards employees as a way of eliciting a level of commitment and 'buy in' that facilitated solidarity of values and long-term relationships. The owner-manager of a medium-sized SME illustrates this when asked if employees display similar values: 
P10: "I think the employees do. They sort of see how we operate and hopefully I think we operate in a pretty ethical way, and I think that's an input from them that, you know, when I say to them how should we handle a problem, they know where we're coming from. Yes I think hopefully it's a two-way thing that they respect us and we respect them really, you know, a bit like family, even like a marriage life. We have our ups and downs, there are not many ... but you try and work through it really". (p. 5, 133-141)

Long-term relationships were favoured by SMEs for a number of organisational reasons. This commitment and longer duration of relations was often found to result in the development of friendships among stakeholders, facilitated by the ownermanagers who suggested they are drawn to stakeholders with aligned values. This process of exposure and progression of relations is described by two ownermanagers below:

P9: "Yes, definitely. I've actually had a time where our customers then become the employees' friends as well, which is nice because they get to know us". (p. 11, 303-304)

P23: "No, it's a bit clichéd, but my clients have become friends. But I didn't bring them in as friends, that's developed through the working”. (p. 11, 296-297)

The long term-orientation of stakeholder relationships ties in with the notions of loyalty and commitment. The development of trust and reputation are key relational benefits that foster long-standing relationships, with socially responsible initiatives often coming as a result of these ties or to strengthen them. One SME refers to the importance of employability, particularly with regards to the accommodation of female employees. Her personal values were reflected in the way she managed her SME, attempting to provide supportive and long-term employment for those employees with families: 
P26: "My values are basically that um...employability, that most of my team now they have been there for me for fifteen years, seventeen years and all have families of their own and without working here, you know, they wouldn't have their wages, I'm very flexible with them, a lot of the team have got families now, so I encourage that...I also encourage, you know for women to be able to juggle their careers and have family balance as well, which is really important". (p. 2, 29-34)

The same owner-manager reflects this caring, maternal nature to the local community as another stakeholder, hoping that her team would replicate similar thoughts:

P26: "We're always working in the community, we're always caring, we're always looking after each other um, so I think the team would possibly have the same thoughts, but this would only be possible if we were still surviving, so it's only a matter of doing both of course”. (p. 2, 54-57)

In order to develop high levels of commitment and retention of staff, P10 spoke of the need to create a positive and harmonious atmosphere for employees in particular. The lack of commission expressed below was rare for sales SMEs of their nature, yet the omission avoided disharmony amongst employees and enabled retention of staff:

P10: "We try and provide a nice atmosphere. We don't pay commission which is one of the...I think that creates a better atmosphere, we're one of the few firms that don't. Because I'm always nervous that if you pay commission it's going to you know, we've tried a few little things and that creates disharmony really". (p. 9, 263-267)

There are multiple benefits as a consequence of long-term stakeholder relationships. One of these is the ability to be reactive to the environment, a huge advantage for the smaller sized businesses. Good supplier relationships mean that jobs can be pushed through quicker than large corporates, giving them competitive advantage:

P23: "If I haven't got an excellent relationship with my suppliers then I'm not going to fulfil my client's orders. So that's really important and that is ongoing that we develop that relationship". 
I: "That is another question, do you have good relationships with your stakeholders?"

P23: "Absolutely. And, like I say, it's been 20 years of streamlining and then nurturing, because there are occasions that I might need something turned round within two days, if I haven't got that relationship, they're not going to push my order to the top". (p. 6, 146-153)

As P3 claims, when trust is reciprocated, stakeholders will be more lenient and accommodating of the SME.

P3: "If your suppliers trust you, you can get away with a lot more [laughter] you can turn a 30 day payment into 60 days". (p. 6, 173175)

Social relationships likened to family and friendships can also be aligned with the responsibility of survival. SMEs are characterised by the fire fighting of tasks and a team atmosphere as facilitated by flat hierarchies. This discards any power differentials in order to empower and treat fairly. Consequently, SME success often relies on relational elements such as respect and loyalty that are nurtured via these team structures. This is referred to in the separate passages below:

P1: "As a small business I think that all of our staff understand that it's all in, so everybody has to help each other out... if the tea needs making everyone helps make the cups of tea, if the phone's ringing we all have to pick the telephone up and I think there is definitely like a respect thing as well”. (p. 3, 86-89)

P30: "We're like a small, like a family here... and that's how I hope we are to staff as well, we're all as one, you're no different to the staff, that's how I see it so yeah, I think nobody is above or below, nobody is above anybody else, everybody just mucks in together don't we”. (p. 1-2, 25-29)

P25: "You are only as good as your employees. So yes, we do like to think, they might not think so, but no, we do like to think that we treat everybody as equal, whether it be someone sweeping the floor or those in charge of the works". (p. 2, 40-43)

Such personal relationships with key stakeholders such as employees align the personal values of the individual with the team and create solidarity with the ethical character and business objectives of the SME. The alignment of morals is referred to 
with regards to the ethicality and competency of suppliers by a small business in the digital marketing industry:

P1: “We've built up a bank of suppliers I guess, that are almost like our family so...they hold exactly the same morals as us in terms of putting the customer first, and we've had some really rubbish suppliers, yeah, really bad who literally just want our money, they don't really care about the quality of work or the cost or anything like that um...but we've always gone back to the ones that have met expectations and sometimes exceed it which is even better". (p. 5, 142-147)

Close relationships such as these also influence the inception of CSR specifically. For example, due to the commonality of flat hierarchies within SMEs, the inception of CSR often flows not only from the personal interests of the owner-manager, but also involves a range of actors close to the SME. One SME facilitates such relationships via the empowerment of stakeholders. When asked whose idea it was to give to charity they responded with:

P1: "We like people to come up with their ideas because then you get the full buy in, you know". (p. 14, 388-389)

Many SMEs referred to CSR ideas initiating from close stakeholders such as employees, as is seen in the extract below by a UK SME that offshores operations to Sri Lanka:

P4: "We've helped with donating older computers that we've finished with to schools and to like charities that are trying to bring technology to rural areas so yeah we've always donated all of our old kit because we recycle kit every sort of three years, so sort of every three years we sort of have got old computers and screens and keyboards and modems and servers and whatever else so that all just goes to local charities or schools and that's completely their idea you know I didn't, they came to me with it and said 'you know, my Uncle's, brothers, cousin's school needs something can we do something', so that's sort of them coming to me with ideas definitely”. (p. 13-14, 387-395)

It is particularly interesting that in this case, the owner-manager in based in the UK, only seeing employees up to twice a year. Despite this lack of physical proximity to employees there is still a level of social proximity and a feeling of moral obligation 
towards the community in Sri Lanka. This contributed to the social welfare of a country thousands of miles away, despite low commitment and lack of cultural proximity. Consequently, employees in this case drove the CSR inception. The openness of ideas from employees and importance of ownership, commitment and loyalty was further evidenced by an SME situated in South Wales:

\begin{abstract}
P6: "A big thing for us is ownership, so our employees do feel a sense of ownership, so it's important that if our employees have an idea for charity events or community nights, it's a bit tricky how it will work sometimes but I'm like let's do it lets give it a go if you're passionate about it and want to run with it, so it's important that they feel like they have a valued say and input you know and they own it a bit”. (p. 13, 377-383)
\end{abstract}

In summary, long-term stakeholder relationships were often a necessity amongst SMEs for business survival. This elucidated benefits for the business such as trust, commitment and loyalty from key actors. The length of these relationships resulted in friendships being formed and stakeholders likened to family. These were often enabled via flat hierarchical structures with equally distributed power internally, something that will be discussed in the next sub-section. This created a common purpose and team atmosphere that enabled the SME to move forward as an aligned entity and deal with the varied nature of organisational activities. There was a strong display of care from owner-managers towards the wellbeing of close stakeholders. In association with this level of care, CSR was often as an outcome of this closeness or used to empower stakeholders as a buy in to encourage such relations. The above factors meant that close stakeholders often initiated CSR, although despite this, decisions on CSR were still very much made by the owner-manager.

\title{
7.2.2: Inextricable Personal and Business Connections
}

In relation to the above characterisation of stakeholder relationships, the findings of the interviews emphasised the inextricable nature of the personal and business in SMEs. These relationships often unavoidably transcend this boundary, creating both advantages and limitations for the SME, with implications for involvement in socially responsible initiatives. One noted benefit perceived as a unique advantage for SMEs was the mutually reinforced relationships. P18 clearly notes that his 
current business endeavours are reliant on close friendships crossing boundaries within his stakeholder network:

\section{P18: "I mean it really is a chicken and egg I could work on my own but not what I do now, I need my employees and a wider network to deliver what I'm delivering so close friendships come actually across boundaries within the network”. (p. 4-5, 119-123)}

He later describes the unique culture inherent in Wales, referring to the importance of good relationships to ensure reputation. A simile is used in the extract below, likening the culture to that of running a village, implying the need to be nice to everyone. This is due to close proximity of individual actors that could potentially be a negative of having complex webs of relations that cross into personal boundaries. This culture, along with the reliance on personal interactions for SMEs, increases moral intensity and the propensity for SMEs to behave ethically.

P18: "Culturally it's very different in Wales, it just runs like a village, you have to be nice about everybody because they're bound to be the cousin of the person you're talking to". (p. 4, 112-114)

There are however a number of benefits of having closely interrelated networks of stakeholders. For example, the positive implications of personal relationships for business were admitted by P12. Because of a developed relationship with suppliers from a previous job, he halved his materials costs, leniency of payment timeframes were also considered by other participants. P12 explains the extent of the tangible benefits incurred as a result of personal relationships:

\footnotetext{
P12: "I used to work for the local authority and I was supervisor and when I finished...I had 23 men under me but I knew the suppliers very, very well right...so when I went self-employed the suppliers gave me a good rate but then I couldn't apply for a Crown contract for three years, so when I got the Crown contract my bills halved". (p. 9, 231235)
}

The personal integration of business relationships was also seen as crucial for business viability. Benefit of these relationship were noticeable for P27 particularly when applying for sub-contracts with large businesses: 
P27: "If you're a small provider, and you want to have sub-contracts with these larger organisations to deliver part of their contract on their behalf, they always take a slice off, like a management fee, but I think having those personal relationships does generate extra business". (p. 15, 431-434)

This was again noticeable whereby personal contacts and reputation of the individual was more important than where the business was physically located. Communication happened via word of mouth within the boundaries of a niche industry. This meant that work was developed organically via personal reputation and close relationships for P19:

P19: "It's mostly word of mouth because the industry...it's quite a niche industry but everyone kind of knows everyone and everyone moves around and stuff, so actually what tends to happen is we'll take on a client and then one of their managers goes to another site in another city and they say, 'Oh you've done a really good job for us, would you like to take on the job here?' So it's almost always organic; we don't do any advertising'. (p. 10, 259-265)

The merging of spheres was further beneficial to business in the access and provision of resources. For example, when hiring people with personal connections, it can reduce risk as the employer has prior knowledge of their competencies and are more likely to align them with the ethical character of the owner-manager and SME. This solidarity will also assist in the assurance of long-term sustainability of the business:

P14: "My teachers were customers before, so I obviously built up a good enough rapport with them for them to want to come and work for me which is nice, a few of the customers have been sort of friends of friends, so it's like word of mouth has sort of been amazing”. (p. 3$4,88-91)$

SMEs' closeness and high levels of commitment to stakeholders influences CSR inception and engagement. As is mentioned above, reputation of the owner-manager is often seen as synonymous with that of the business. CSR can strengthen personal connections with stakeholders and legitimise the business via the nurturing of trust and reputation. The personal connections of the SME can also influence the CSR engagement of their stakeholders. For example, the owner-manager below uses her personal connections with charities and links them up with her clients: 
P20: "I do a lot of things for charity but I was doing that personally anyway. In fact now I am linking in with all like, I link charities with clients but I also do, so for instance was doing a walk with one client and I will do that with them. So I am basically working with about six different charities at the moment with my clients, but all of them I know, so like I know the contacts personally, I know the contacts from the charities". (p. 13, 352-358)

This multi-stakeholder perspective of CSR engagement was common with SMEs, noticeably influencing and influenced by their complex webs of relations. The above examples are evidence of how the complexity of personal and business relations can impact CSR. However, there are potential negative implications of such closeness to stakeholders. Such affective relationships may cause moral dilemmas or cognitive dissonance because of their partiality towards those stakeholders that transcend the business boundaries into personal. The ownermanager of a medium-sized company displays such conflicts of interest in the following extract:

P10: "When staff become your friends and colleagues it's very hard to reprimand them... I do quite often turn a blind eye to things, you know. If it was my daughter I would tell her, and that's not fair, you know... whereas it's more the long-term employees you know their shortcomings, it's not worth it. But that's not good for the business either really". (p. 6, 163-169)

This conflict of interest between the responsibility of business viability and commitment to stakeholders from both spheres can be seen to create moral dilemmas for the owner-manager in adhering to their business responsibilities. This was again explained by P22 who would have sacrificed business responsibilities in order to save family connections. This partiality to family is shown below:

P22: "There's been a family link, obviously, with my dad because he, you know, I used to work for him then he worked for me and there was ups and downs with that as you can imagine. That, in fairness, that is quite a relevant question really in terms of looking back over the years because that was a double-edged sword very much with the family, very difficult. It became a potential nightmare and I think in the end we both had to part our business ways or risk losing family, which is more important to me". (p. 16, 421-428) 
As P23 explains, naturally personal life is merged with business when it comes to SMEs, describing it as 'blurred lines':

\section{I: "Is your family life integrated or completely separate to your role} as an owner-manager?"

P23: "I try and keep it as separate as I can, but naturally it's integrated, absolutely, everyone wants that. For three years I worked from home because my little one was part-time schooling, as soon as he went full time, that's when I said I've got to get out, I need an office, because then it was just all blurred lines". (p. 10, 275-281)

A further example was the employment of a staff member via a Jobs Growth Wales scheme. The member of staff was not proactive to enhance business and therefore the owner-manager was paying their wage and compromising their own. This was because of the personal connections and level of commitment to their employee. A moral dilemma occurred in this instance impacting the responsibility of business survival, it resulted in them pursuing a mutually beneficial solution to the problem as seen below:

P11: "I um employed a member of staff but she wasn't getting enough business in but because I've nurtured her from leaving college I wasn't taking any pay so that I could pay her, so now I'm in a really difficult position and it's been going on for a while so I've got to the point where I'm just going to have to sit her down and discuss it um, I've thought long and hard so what I'm going to propose is that she goes on a self employed basis". (p. 1, 16-21)

Such dilemmas can also be translated into CSR engagement. This was evident in the focus group findings whereby CSR activities were often driven by the relational pressures of proximity. For example, P15 was discounting for family and friends which was compromising the sustainability of a viable business:

P15: "I've been a lot more careful recently because when I first started out I was saying to people yeah it's supposed to be £20 but you can have it for $£ 15$ and then when I actually sat back and looked at the customers I was selling to, it was mostly friends and family in the very beginning, at which point I was barely making any money on it and made it pointless". (p. 9, 240-244) 
As P4 explains, it can be really difficult to say no to friends when they ask for advice or the donation of time and skill:

\begin{abstract}
P4: "I also have a kind of well, they call it mates rates, so if somebody, a friend needed something doing then I would do it at cost or you know very little profit so, that's something that I definitively do um and I think I often help and because you know I'm in that kind of tech sector, often people will just ask you for advice and help and I find it very hard to say no and I will do it even though I'm not going to get any money out of it”.' (p. 12, 354-359)
\end{abstract}

There appears to be a cognitive dissonance between the responsibility to survive as a business and commitments to friends and family. Such closeness can even deter SMEs from engaging or communicating about CSR efforts in fear of request escalation. This lack of communication may inhibit dissemination of knowledge and limit conceptual awareness of CSR for SMEs. Issues of escalation of CSR requests are considered y P13 below:

\footnotetext{
P13: "I suppose sometimes you can open the floodgates if you start to be quite, if you start to support too many causes you get a lot of requests then". (p. 19, 488-489)
}

As has already been said, owner-managers find it difficult to say no to stakeholder CSR requests. It can have implications for both their personal and business reputation, something that could quickly diminish via word of mouth. P26 uses the word 'plagued', implying negative views of the constant request for sponsorship:

\begin{abstract}
P26: "We do get plagued... and I find it very hard to say no and I wouldn't want them to be negative and you know...but they don't realise that I've already said yes to thirty other people, so it's that one person that might be miffed, or they didn't get a response, because as a small business if we said yes to everything it's really challenging”. (p. 4, 92-96)
\end{abstract}

In summary, it seems clear that the inextricable personal and business connections are both vital but challenging for SMEs. There appears to be a reliance on friendships crossing into business networks, what we can assume is a result of the embedded 'village' culture of the research context. Owner-managers and stakeholders are therefore likely to be linked by more than one kind of relationship. 
There are numerous tangible and intangible benefits of this boundary being merged, reducing costs, assisting business operations and opening up doors of opportunities with other businesses. CSR both influences and is influenced by the mutually reinforcing personal and business sphere connections. This often results in multistakeholder approaches to CSR engagement. However, close associations can cause challenges. Moral dilemmas were created due to the tensions occurring between the business responsibility to survive and partiality to those with personal connections, on many occasions this was associated with the escalation of requests for forms of CSR. Owner-managers most commonly sought symbiotic solutions to these dilemmas.

\subsection{3: Community Embeddedness, Reputation and Word of Mouth}

The merging of personal and business spheres for SMEs can further be explained via community embeddedness, as was also apparent in the emergent focus group findings. SMEs do not operate in isolation; they are embedded in webs of social relations within communities, building relational attributes such as trust and reputation via personal contacts. This contributes to sustainable business and ensures a form of relational embeddedness, in addition to geographical. These key assets of community and relational embeddedness play a big role in enabling mutually beneficial relationships and shared resources. Proximity ultimately shapes CSR engagement between SMEs and their stakeholders and is likely to be the reason for SME owner-managers commonly conceptualising CSR as a community based phenomenon.

However, community is being redefined by businesses due to a number of reasons. There were dispersed accounts as to what and whom SME owner-managers considered as their community. The traditional view of physically located SMEs is changing due to technological developments and globalisation, with increasing numbers of businesses operating online. This redefines the concept of community embeddedness and closeness to key stakeholders. This is likely to influence the relational networks of SMEs, the ethical decision making of owner-managers and their consequent CSR efforts. This view of not having what would typically be considered a physically proximate community was demonstrated by P3. He initially 
proposed that his SME did not have a local community as it operates online; this was reconsidered to constitute his key customers as the local community for the SME:

I: "How would you define your local community in terms of geography or distance?"

P3: "Yeah, so geography doesn't really come into the, into the equation, it's um it's wherever customers may be based”. (p. 7, 185191)

He further went on to say:

P3: "I don't really have a local community I don't know if it's...okay, so my local, lets say the biggest part of my recruitment work is probably at a graduate level so, yeah top five universities in the UK I do a lot of work with so that's my local community". (p. 7, 181-184)

P18 had conflicting views regarding his local community. At first he referred to it as being anyone whom his work impacts, then retracting and referring to that as social responsibility. Community was then described as people within physical or social proximity, whom he knows and meets with or knows of via some form of connection within his personal or business networks:

P18: "You see that little lad I'd never seen whose father drove our car in the Middle East, he's my local community because he's...basically my local community is anybody and everybody upon whom my work impacts, that's a lot of people in one sense but in terms of you know, when I say community I'll sort of focus it down on those I know or know of um, I'm aware of, I mean I put it as social responsibility among other things, rather than community responsibility, social is everybody generally, community is the people I, actually the people I meet with or essentially meet with”. (p. 7, 197203)

The implications that this changing perspective of community has on the ethical decision making process of owner-managers and overall CSR efforts is unknown. P21 takes a view similar to P3 and is similarly operating her SME online, she considers customers nationally as the community. She expands on this by saying that regardless of where their customers are located, it does not make any difference 
to the way that they are treated, implying that perhaps it is not just physical proximity that increases the propensity to behave ethically.

P21: "Yes. I get the sense of...I would say that we consider that community, I would say that we consider our customers nationally a community, and I think that it doesn't make any difference whether someone was in Swansea or if they were in Essex as to how I would treat them". (p. 11, 296-299)

One thing that has been reinforced in the above passages is the changing paradigm of community for SMEs. This has implications for embeddedness and the relational ties for SMEs. That being said, there was still a strong preference for SMEs to support the physical locality of the business, particularly sourcing from local suppliers. This supplier and community directed form of social responsibility stems from a sense of duty to support the local economy as seen below:

P23: "People particularly in South Wales I think, like to buy local. They do feel that they're supporting the economy if they do". (p. 9, 256-258)

Owner-managers identification towards the locality and desire for the area to prosper meant that they saw value in keeping money within the local economy via the utilisation of local supply chains. P9 and P7 both refer to this as being part of the CSR engagement:

P9: "The main thing we try and do is keep everything as local as we can so we use like the [local SME] and the just everything we try and do I always look at how far they will travel to deliver to us and back, the footprint as such. As the owner I'm based in Swansea as well, so it's keeping the money in Swansea, it's making a profit for Swansea". (p. 1, 14-18)

P7: "Everything that we make we put back into the business to grow it, obviously we're quite small now but in the future as we try and employ people there will always be from Swansea and we will try to keep as much of our trade as we can in Swansea to money back into the local economy". (p. 1, 15-18)

P16 further expands on this and explains they would much rather buy local, even if it is an online SME who they hadn't met before: 
P16: "I'd much rather buy from a local company, even if it's online, there's a company that I buy a certain thing from and even though I haven't met them but I think well they're local so I think well I'll buy from them rather than someone from England". (p. 5, 134-136)

The importance of retaining employment and money within the local economy is made explicit by the presentation of the statistics below. This ensures economic sustainability of the region and contributes as a social responsibility, P27 explain how in terms of the delivery of public sector contracts:

\begin{abstract}
P27: "Something like 70p of every pound spent from a local small company, delivering a public-sector contract, it generates huge benefits because all of that money is retained locally, whereas if you've got a large national charity, for example, delivering a contract, I think only about $43 p$ in the pound stays in the local economy because it all goes to head offices and leaks out of the area". (p. 4, 95-99)
\end{abstract}

The use of local stakeholders was considered as part of the ethical thing to do, although there were numerous benefits considered as an outcome of this. It allows the nurturing of relationships, becoming mutually beneficial for both parties and the local area. As the owner-manager below demonstrates, relational embeddedness is advantageous for business operations and provides distinct advantage for SMEs. The example below refers to favours and pushing jobs through on a quicker timescale:

\begin{abstract}
P22: "I do believe in kind of keeping the money in our local economies. So, and I think we do like to maintain these relationships but sometimes if you need a print job or you need a favour and you know there's people you can turn to and say, 'can you push this job through really quickly?' and they will do that and they kind of do it for you, so, that's a nice thing to be able to rely on". (p. 12, 309-316)
\end{abstract}

The unique benefits of being SME closely embedded within the community were considered as a special defining feature of SMEs by P30. This allowed them to assist their customers on a more caring level with small tasks: 
P30: "There's quite a few customers that ask us to help them with their fuel and because you're small you can deal with it can't you, so yeah it's just a small community isn't it and those little things make a difference, well I think so anyway”. (p. 5, 133-136)

A further benefit of embeddedness within the community is the communication channels through personal contacts. This is implied in the following extract whereby the SME claims to rely on social webs of relations for business growth:

P29: "I think our business definitely relies on social elements of like friends of friends for growth and stuff, but then obviously we're doing favours for people in the community they'll say 'my accountant does this for me and doesn't charge us for the advice' so we're doing a free service and it does come back to help us in the end, but we don't do it for that reason specifically”. (p. 24, 680-684)

CSR activities in this instance reinforce embeddedness and legitimacy of the SME by strengthening the perception of the business as trustworthy and a responsible societal actor. One owner-manager demonstrates this improved perception and communication of the business below:

P7: "There's a word for it, the more good stuff you do like the more good stuff will come into your business, yeah there's a word for it, but the more we put out to the community, not like I don't think we expect anything back from it, but we just think it does have an affect in terms of our business". (p. 6, 157-161)

The above participant is referring to word of mouth as a communication channel that can develop or diminish moral reputation, business competency and trust, particularly when embedded within the community. Many SMEs rely on word of mouth for the retention of business and growth. This is demonstrated in the three individual extracts below:

P12: "Word of mouth is all my work". (p. 2, 48)

P29: "We don't advertise, like we've got ten new clients in the last month and they're all through word of mouth". (p. 13, 360-362)

P3: "Reputation I think has certainly stuck with me for the past ten years, so all my business comes through referral”. (p. 2, 48-50) 
The reliance on word of mouth, referrals and relational marketing channels are dominant in SMEs due to their unique characteristics. For example, limited residual profit for formal marketing budgets, preference to retain current customers and grow organically, characterised by the ad hoc and informal nature of contacts. However, social interactions and recommendation via informal channels are not limited to small-scale deals and contracts, but can also obtain large contracts for SMEs:

P22: "We have had some huge contracts in the past often through, by accident; I say by accident, through recommendation and being in the right place at the right time or knowing the right people". (p. 18, 489491)

Word of mouth is reinforced as the best form of recommendation and can actually be considered a saviour for SMEs during times of economic downturn. This is when trust in the economy and society are generally weakened and there is a need for reinforcement:

P22: "It's a lot easier to keep your current clients happy and keep them coming back for more work and recommending you as well because that's an advert in itself, word of mouth is the best form of recommendation".

I: "Yes, word of mouth. That's the one thing that everyone has said is word of mouth".

P22: "Yes, absolutely, absolutely and that's always been our saviour really, I suppose, when you're doing recessions and things like that because we've been through two big recessions since I've been involved in the business and I think that has been one of the biggest things is that at least even when the chips are down customers know that they can trust us to deliver and that we're going to be there and will recommend us". (p. 2, 33-44)

The SME above did not have the resources for a marketing budget. Consequently, there is a reliance on word of mouth and a preference to keep current clients and repeat business. This was beneficial during economic downturns, as the SME did not require a high turnover of customers. Interestingly, one participant explains that her SME is more reliant than most on word of mouth due to a lack of physical premises such as a shop front and proximity to the community as a business: 
P11: “...because I don't have a shop front I rely heavily on word of mouth so for me marketing is a big big thing and making sure that the customers are satisfied". (p. 1, 13-14)

However, there is also a dark side to word of mouth, intensified by community and relational embeddedness. As P1 explains, interlinked channels of relationships that facilitate SME success can also diminish reputation:

P1: "What we don't want to happen is one of our customers to be unhappy with what we've done and then go and tell all their friends, because that would just diminish reputation". (p. 4, 93-95)

As P9, P18 and P24 describe on separate occasions, reputation and trust can be volatile in terms of taking years to establish and seconds to diminish, particularly due to embeddedness and the inextricable personal and organisational contacts:

P18: "It takes years to build a reputation and you can throw it away in seconds with the wrong remark, the wrong presentation of the right remark but you have to be honest, you have to be straight with people”. (p. 2, 48-50)

P9: "I always find that if someone has a bad experience they will tell all their friends, but if they have a good experience they won't tell that many people, it's quite like that”. (p. 3, 67-69)

P24: "Reputation I would say is so important, if we help others then we look good to other people outside then. With the business reputation it is a big thing, so if you get one bad thing said about you it does spread, especially in a small town like this, so yeah I suppose that is just to give us a bit of a good reputation". (p. 4, 105-107)

The final benefit that community embeddedness facilitated was the collaboration of CSR efforts for SMEs. For example, large charity events involving multiple local SMEs working together, this also helps develop or foster current relationships as a force for good in the community. This is described in the extract below: 
P26: "Last week, I did like a charity dinner in the golf course for like sixty ladies there, next week we're doing a fashion show and that's using all the different businesses around Mumbles we have been working together and so...yeah I do, do a lot, I think that within a month, there must be at least two or three big events that I do to support"'. (p. 3, 78-82)

However, it could be said that on occasions the personal nature of CSR efforts and community embeddedness can be inhibiting. For example, the owner-manager may not be open to CSR suggestions from key stakeholders or those stakeholders may defer from approaching the SME due to a known association with a particular cause or charity. This is apparent in the extract below:

P23: "People don't really contact me, because again the community, it's very known I sit on the fund-raising committee for Paul Popham Fund, so any ... so I support them. So for example, any merchandise they have, they get at cost and I give my expertise to them for free and I put it to my clients that we name Paul Popham as their charity of choice. So, yes, I'm not really contacted in that respect, because they know my alliance". (p. 12, 317-322)

This may limit engagement with a diverse range of potential CSR initiatives that contribute to society on a wider scale. As previously mentioned, SMEs are often time limited and wait for CSR opportunities rather than proactively seek them out, this may result in more limited CSR efforts.

One interesting finding was from an SME that operates via an online platform. Their perspective was that the nurturing of relationships for online businesses is even more important for both reputation and the personification of the brand:

P23: "There is a mix. I've got clients that I've never met, but again, we can over-service and develop and nurture that relationship, whether it's through social media, because that is a big one. And, again, with being an online, you've got to have your online reputation. And, of course, everyone's Googling reviews now and so I think that's in line. But we, particularly because the industry we're in, we find that sending them something, personifying the brand, I think that's what's important when you don't get to see a client. So that they still feel connected to the brand". (p. 9, 232-238) 
They further go on to explain that despite no personal interaction, alliances can be built over email and strengthened via CSR gestures such as small gifts. This was again seen to be positive in terms of reputation via online social media communication channels such as Twitter:

\begin{abstract}
P23: "There was no personal interaction, it was all done on over email. And, again, I had another one yesterday enquiring through the website, so it was all done online. But we just sent them a pack of Welsh shortbread cookies with a card saying, 'Hope you enjoy these as much as we've enjoyed working with you.' They've taken to Twitter then. So, again, you've just ranked yourself there. So you're buying their loyalty by...and that's what we believe we can personify the brand even if you're not there, then there's an alliance there". (p. 9, 242-249)
\end{abstract}

On the other hand, P19 ran into the difficulties of being geographically dispersed. In terms of adhering to their social responsibilities, the provision of support to employees when not physically close was seen as challenging. However, the consciousness and guilt implied by the owner-manager suggests that they are ethically conscious of their responsibilities towards employees:

P19: "I'll have staff in places like Sheffield for example, where it's a three and a half hour trek and it's really hard to support my photographer up there, for example. I mean, he's great, he sort of cracks on with it, but I do often feel like really guilty that I can't sort of pop up to make sure he's alright and see how he's getting on". (p. 20, 533-537)

Despite these implications that SMEs still feel a duty to behave responsibly to those outside of physically proximate communities, there was an implied impact on the inception of CSR. This was when SMEs did not have physical business presence in the community. As P18 explains, without a physical shop front, people are less inclined to approach the SME and drive the inception of CSR:

P18: "I don't have premises, I think if I had a shop front with [SME name] people would knock on the door and say could you sponsor my...football team or whatever and I would reasonably consider it". (p. 9, 253-256) 
To conclude this section, the changing structure of community embeddedness for SMEs is something that needs to be acknowledged in future research. Whilst SMEs CSR efforts are still influenced by embeddedness within physical communities, it was noticeable that this has and is changing with time. Proximity and embeddedness within the community is likely to increase the propensity to be ethical and increase the costs of being unethical, it also diversifies options for involvement in socially responsible initiatives. However, proximity was not just physical, when SMEs' are operating online their communities are often considered industry, countrywide or international. Despite the lack of physical closeness, owner-managers still displayed ethicality towards stakeholders, often intensifying the importance of CSR gestures to overcome the physical boundaries. However, there does seem to be limiting implications on the inception of CSR when there is a lack of physical presence within communities.

\subsection{4: Networks and Collaborations}

Whilst this theme transcends the boundaries of others, it is worth distinguishing. SMEs in this context were increasingly reliant on networks and collaborations, both for business and CSR efforts. With increasing stakeholder expectations and competitiveness, this reliance is only likely to increase. The utilisation of collaborative environments often results in reciprocal relationships that create synergistic environments for both parties, enabling the sharing of knowledge and resources. The importance of collaborative efforts is considered by P7. This aids the SME survival as a collective and contributes to localised economic success:

\section{P7: "Small businesses shouldn't compete with each other they should support each other and then like compete against the big businesses, because I just think that small businesses are really good, small business is better for the economy, like if everyone shopped with the small businesses". (p. 5, 146-149)}

This theme of collaborative work and mutually beneficial relationships was replicated by a number of owner-managers. These were identified as being with internal stakeholders such as employees: 
P26: "Yeah, I think that um, it, it's got to be a partnership and it's got to be down to communicate so well with the team that, if I gave so much as a $£ 1$ for them, they would also participate and return that value back, so it's like a two way relationship really and it works". (p. 2, 39-42)

However, partnerships were also made with external stakeholders such as suppliers and even competitors. The below extract refers to the seeking and development of local supplier partnerships, personal relationships and the desire to create mutually beneficial bonds:

P6: "If people are doing good produce, like a small company, then doing good quality stuff and they're local like we love to have them in, so we want to make partnerships with them as well so we're looking at a possible [local supplying SME] and we just love them as a couple as they put so much into their work, it's really important to try and like boost them as well, they help us and we help them". (p. 9, 246-251)

SMEs unique perception towards competitors is that remaining friends and distributing work evenly is more important for the local area than hostile dealings with business competitors:

P22: "Going back to the days when the council used to give us a lot of work, I mean, their budget's been slashed drastically over the last years but, you know, we were always happy, we used to tender and pitch for jobs a lot and I was always happy to see it spread out amongst us and other agencies in Swansea because I think there is enough work to go round and I'd rather they spend it equally with all of us and us all be friends". (p. 17-18, 476-481)

For one owner-manager there was an appreciation towards both suppliers and competitors and an understanding that most have similar goals in collectively building business for SMEs. It was also said that actually, those external stakeholders that are not open to building relationships and collaborating will struggle in the future as times and expectations are changing. This is referred to in both of the extracts below: 
P5: "I do have a relationship with competitors, because that affects you know to be together and to sort of really help push tourism then we get more customers". (p. 5, 127-128)

P1: "If they're trying to do the same thing then that is great because it just means that there's more business going on for small businesses in the areas, it's just generating more entrepreneurs. But there are some suppliers and some competitors that actually, are very closed and I think those are the competitors that could struggle in about 510 years time because they are not open to relationships and collaborative working”. (p. 12, 345-350)

As the times change and there are increasing expectations, the creation of stakeholder networks and collaborations facilitates both the sharing of resources and problem solving. For SMEs who are commonly limited in their access to professional services or with potential knowledge gaps, these networks are invaluable. P27 describes their stakeholder relationships as reciprocal in the sharing of information and problem solving:

I: "If you needed help with something, would your stakeholders help you back?"

P27: "I'd pretty much say so, yes, because there's lots of information sharing, problem solving and so on". (p. 21, 590-593)

One female SME owner-manager actually created a network of female businesswomen in order to provide a network of support that provided a diverse range of knowledge and expertise. The value of this network for women in business in her local areas is described below:

P23: "I set up the Swansea Women's Soiree about five years ago and it's basically a network of local business women. But they have been instrumental because they're all different disciplines. So, for example, I had an issue with insurance. I was able to speak to someone within that network who was able to support and signpost”. (p. 7, 174-180)

She further goes on to say how the soiree, as in informal network, has been invaluable for many women business owners: 
P23: "It's a very informal network but the soiree has been invaluable and we didn't see that when we set it up, but it really has". (p. 7, 190191)

Many SMEs displayed a preference of collaborative relationships in order to create synergistic working relationships. SMEs displayed a sense of duty towards other SMEs in order to work collectively and compete against larger corporate businesses in this country. Collaboration was also seen as a feature of CSR engagement, this has already been shown above with regards to community events but is also evident amongst key stakeholders and those within physically or socially close business networks. For example, SMEs and suppliers working towards the same charitable cause as seen below:

P21: "My printers have really helped me with stuff over the years. So for example the Believe in Magic stuff for Meg, this little girl's charity, she's really poorly herself, and she set this charity up. And they've often printed stuff for her for free, so yes they've kind of done that'. (p. 26, 704-707)

This collaboration of CSR efforts was also seen by P1 whereby the customers and SME decided to donate money to charity rather than gifts at Christmas time. The clients then chose which local charity the money would go to as seen below:

P1: “...as part of the society we um, kind of participate in a lot of um charities, so at Christmas so, at any point actually, Christmas as specially, we, this year we are donating all of the money that, we would normally give our customers gifts, to two local charities and our clients actually get to choose which charity that money goes to". (p. 1, 6-10)

The responsibility to be ethical as an SME was characterised by networks, relationships and collaboration with a view towards mutual support. Ownermanagers, for example P18 below, demonstrated this synergistic view towards relationships and networks on numerous occasions:

P18: "We work in partnership, we're all niche businesses so working together so that collectively we can bring a bigger offering um but we, there are jobs that we do on our own and jobs that we do together and having the flexibility for that is great". (p. 5, 132-134) 
This is particularly important to adhere to increasing stakeholder expectations. With regards to CSR, expectations from society and key stakeholders do seem to be increasing exponentially. Some aspects of what would be considered CSR are therefore now being expected. P23 demonstrates this below:

\section{I: "Do you think that if you asked your stakeholders or the recipients of CSR do you think that they'd reciprocate?" \\ P13: "I think so, I think some of them would, yes. I think a lot of them are customers and so they see it as your responsibility to support them anyway". (p. 19, 477-478)}

SMEs in this research are increasingly relying on networks and favouring collaborations for both business and CSR efforts. Collaborative networks of stakeholders allow synergistic environments and mutually beneficial outcomes. SMEs have the mentality that they need to stick together and support each other, often resulting in unique alliances and collaborations. These were not limited to internal stakeholders, but also external stakeholders including competitors. The equal distribution of work amongst a community was favourable, this was claimed to collectively assist the survival of SMEs. Those businesses not open to collaboration may struggle as the times are changing with expectations from stakeholders intensifying. Increasingly, working amongst networks and in teams is essential to adhere to these expectations and essential for the sharing of resources, problem solving and knowledge dissemination. This also translates into collaborative CSR efforts, with teams of stakeholders, often from the same location, pitching together to support causes.

\subsection{5: The Dynamic Nature of SMEs}

One profound finding was the context dependent, dynamic nature of business for SMEs. The acceptance of owner-manager identification, business operations and stakeholder relationships as multiple and changing is key to understanding CSR. Such fluidity is beneficial in terms of the ability to be reactive to the environment to enable business viability. However, this adds to the complexity of understanding SME behaviour and the true origins of what truly drives the inception and 
engagement of CSR. With regards to business operations P27 refers to the multitasking nature unique to SMEs:

P27: "That's what ends up happening with a small business. You do finance, HR, health and safety. Last week I had the insurance broker coming out, because it's that time of year and even down to trivial little things like the other week I ended up running down the shop, because we're out of post-it notes, yes, honestly”. (p. 5, 137-140)

This dynamic nature of business was regarded as a unique benefit for SMEs, as P27 continued to explain:

P27: “...we are a lot more responsive and I think that's one of the main strengths and unique selling points really. Whereas we can get, if someone said, 'oh I've got this, would you be interested in doing this new programme tomorrow?' We'd have a job advert out the same day, job description written, you don't have to faff around with bureaucratic internal processes". (p. 6, 160-164)

The above extract demonstrates the dynamic nature of business operations. This is considered beneficial for SMEs to be responsive to opportunities without 'bureaucratic internal processes'. The changing environments and constant adaptation that businesses are faced with can make it difficult for SMEs to prioritise their stakeholders and responsibilities. These are likely to change in prevalence temporally. P1 explains this difficulty when talking of the prioritisation of responsibilities:

P1: "It is difficult to prioritise them but...like I said, every day is different and the priorities are going to change on a daily basis". (p. $8,222-223$ )

The dynamic and fire fighting nature of business operations within SMEs is also evidenced by P30. She explains that they deal with each day as it comes, with not two days the same:

P30: "It's not prioritising, it's just dealing with day to day as it happens don't you, this place is never, no two days are the same...definitely, even with the forecourt no two days are the same". (p. 1, 4-6) 
As P10 explains that habit can be dangerous for SMEs, business is context dependent and it is important to be open to new ways of doing things, being responsive and reflective to continually changing criteria. This openness facilitates innovation and innovative ways of doing things:

P10: "I think it's really important to reassess things and actually not have a set pattern sometimes, because the criteria always change. Or perhaps the priorities of the individuals criteria change". (p. 15, 413415)

This multitasking nature of SMEs is often reflected in CSR engagement. It was most commonly characterised as ad hoc and without strategic intent. There were very limited occasions whereby owner-managers formalised CSR efforts, or even communicated. This is evident in the below extracts:

P28: "We don't really keep a log of it, it is just what it is really". (p. $7,186)$

P22: 'It just happens, it happens. If I've got the time to spare and it's sporadic, it's not like I say right I'm going to spend six hours a month doing this or sometimes like lately I've been working for [name of CSR recipient], I'd probably spend a couple of days working because it's, you know, we know that there's a lot to do at the moment and he's been launching a brand but it might go two or three months with nothing at all, so if I can afford that time I just weigh it up in my head and just balance it and do what I can". (p. 28, 772-779)

A number of SMEs did try to estimate or budget their CSR efforts. However, this was dynamic and not fixed, with participants explaining that often these predicted amounts are not often adhered to:

P26: "We do have a rough budget, a rough guide to go by, that we sometimes smash but [laughter], we initially start off in January with a budget”. (p. 5, 117-118)

As P2 explains, in his SME they would budget for the opposite reason of controlling and limiting CSR expenditure, but instead to ensure that it is being spent and the business is giving back to their community: 
P2: "We're going to budget it because it has been ad hoc and it's usually so that we budget it so that we spend it rather than that we don't because a year can go by and we could go well what have we done, we've done well but what have we done for anyone else?" ( $\mathrm{p}$. $10,272-275)$

Interestingly, P10 refers to the collaborative nature of CSR discussed in the above sub-section. He believes that as a business, they cannot ask too much of those supporting them. It is appreciated that they may have their own social responsibility identity or pursuits of a different nature or for a different cause. The below quote therefore acknowledges the personal, context dependent and dynamic nature of CSR:

P10: "It happens ad hoc and it's more sort of we're looking for donations, whether it was money or product. But like all these things you can't do it too often because, you know, you can't ... you know, sort of bite the hand that feeds you sort of thing. I mean we could do it but we can't really ask suppliers to do it very often'. (p. 25, 691695)

P10 further explains this by saying:

P10: "When I was High Sheriff I gave quite a bit more but then, you know, you've asked a lot from suppliers, you can't go back the next year and do the same. They've got their own social responsibility and they might be going in a different area really I think". (p. 28, 768-772)

These findings reinforce the complexity of SMEs and the dynamic nature of business operations. This influences the way that responsibilities are prioritised and ultimately how CSR is enacted and engaged with. Influencing factors of CSR are constantly adapting depending on changing personal interests, merged spheres of stakeholder relations, business operations and the external environment. In order to better understand this complex context around each CSR and their socially responsible initiatives, these findings call for context specific research, exploring these changing factors and acknowledging them as fluid and not fixed.

\subsection{6: Stakeholder Relationships and the Balance of Power}


While stakeholder relations in general have been analysed earlier, an additional concept to discuss is the distribution of power in SME stakeholder relationships. This was dependent on the specific stakeholder, their importance and closeness to the business. The preference of SMEs to employ flat hierarchies to structure their business has already been touched on, but this form of empowerment was a common characteristic of SMEs. P19 and P30 consider it their responsibility to support their employees and discard any power differentials with staff to empower and treat them fair:

P25: "Yes, you are only as good as your employees. So yes, we do like to think, they might not think so, but no, we do like to think that we treat everybody as equal, whether it be someone sweeping the floor or those in charge of the works, we do like to treat people and we treat everybody fair". (p. 2, 40-43)

P30: "We're like a small, like a family here... and that's how I hope we are to staff as well, we're all as one, you're no different to the staff, that's how I see it so yeah, I think nobody is above or below, nobody is above anybody else, everybody just mucks in together don't we". (p. 1-2, 25-29)

One the other hand, SMEs often described examples of having imbalanced relationships with external stakeholders dictating. For example, P21 have learnt by experience to diversify their client base so as not to put all eggs in one basket, as they did in their early business years. They describe this below:

P22: "We've got a good batch of long-term customers which is nice who have stayed with us and varying degrees of signs of clients, you know, we've got some that are multinationals and then, run on values. So, some people will use us once for $£ 100$ job and we'll never see them again. Somebody else will spend, you know, thousands every month with us so it's really varied client base, to be honest. There's no one, there's no eggs in one basket as such which we, it has happened in the past where we've had very big clients that have kind of dominated the others which is always a bit scary because we think if they go for any reason, you're kind of, you've got, you know, it's a big void to fill but luckily as I say, people stay years so we haven't seen a problem but yes, it's a very varied list". (p. 9, 242-251)

Even as a medium-sized business, P27 still has one main customer who monitors the progress of any jobs that are being taken on by the SME. This monitoring process 
suggests that the large business is a powerful stakeholder and therefore the success of the SME is defined by their relationship with them.

P27: "We do have one main customer, a company by the name of EF Global and they probably do give us $70 \%$ of our work".

I: "What's the balance of power like between you, do they dictate or do you have a good balanced relationship?"

P27: "We do have a good relationship with them. So, obviously they do dictate delivery dates but yes, we have to give them a one-week work progress sheet, every week so they can see exactly what state their jobs are at. Whether we're on time, whether we're late and so we can discuss things every week and assist them and we know all their job are going through the shop". (p. 5, 110-118)

P3 suggested that some clients do perceive that their importance is higher than it is, with the perception that they own the SME. P3 however denies the truth in this:

\begin{abstract}
P3: "You have to prioritise everything you do to meet the clients expectations but you have to juggle their expectations as well, some clients can perceive that they own you, or that they are the strongest in demand but you can quite quickly go back and look at, you know some of the metrics, the stats behind performance and realise that you know the return on investment is so minimal that actually they should be an insignificant part of your business day”. (p. 5, 125-131)
\end{abstract}

The reliance on stakeholders was again evident when P23 was let down by a supplier. Consequently there were legal implications for the SME as customer orders were not delivered. Negative experiences such as these show the dangers of reliance on stakeholders on delivery to customers, but also the importance of relationships.

P23: "I ended up in court two years ago, because I got let down by a new supplier. So, of course, that is something that I am quite risk adverse when it comes to that. But they needed something urgently, the supplier I'd normally use I couldn't. I worked with them, they let me down on a grand scale”. (p. 6, 166-169)

Examples were also infused throughout the previous themes whereby SMEs were defined and confined by their embeddedness within communities and the transcending of business and personal spheres. The most important point to take 
from this theme is the differences in power dependent on the different stakeholders. In the above examples there was evidence of SMEs diffusing power differences with internal stakeholders, yet being dictated by relationships with dominant customers or the reliance on suppliers to deliver on time. This has a direct impact on their responsibilities, for example, to survive and abide by the law. SMEs do appear to be defined by their relationships with powerful stakeholders. The multiplex factors that contribute to their associated power is one that is in need of further exploration.

\section{3: Chapter Conclusion}

To conclude this chapter of organisational level findings, human actors are inextricably embedded in context and relationships with stakeholders are inherently complex, transcending both personal and business spheres. In answering the key questions, the influence that stakeholder dynamics has on the inception and engagement with CSR is difficult to articulate, with additional complexity due to the continually changing community and business landscape over time. These findings call for recognition of the context specific nature of SMEs and acknowledging them as fluid, not fixed entities.

It is obvious from these findings that the transcending of the personal into business is commonplace for SMEs. There is a reliance on friendships either crossing into business or being developed as a result, however this can be paradoxical for the SME, creating moral dilemmas for the owner-manager. CSR both influences and is influenced by these characterisations, often as an outcome of personal links to actors. However, despite the SME taking inspiration from close stakeholders for the inception of CSR, the end decision most often came back to the owner-manager. This has interesting implications in that CSR engagement is likely to always refer back to them as a central actor.

Whilst SMEs CSR efforts are still influenced by embeddedness within physical communities, it was noticeable that this is changing with technological developments and globalisation. SMEs operating online often considered their communities as industry, countrywide or international. Despite this lack of physical embeddedness, 
owner-managers were still ethically considerate towards stakeholders, often intensifying the need of CSR to nurture relationships. However, despite no evident implications for engagement, there does seem to be limitations on the inception of CSR when a lack of physical existence within communities.

SMEs are increasingly relying on collaborative efforts for business and CSR. Networks facilitate synergistic environments and mutually beneficial outcomes, providing support and creating alliances with other SMEs. Collaborative efforts are needed to meet the increasing demands of stakeholders and those businesses not open to collaboration may struggle in the future. This also translates into collaborative CSR efforts, with teams of stakeholders working together to contribute to particular causes. These were philanthropic in nature; whilst not denying the valuable contribution, the sustainability of such initiatives could be questioned. Further, there were no collaborative CSR instances in this research with geographically dispersed SMEs, an area that could be of interest in the future.

These findings reinforce the dynamic nature of SMEs; this influences the way that responsibilities are prioritised and ultimately how CSR is enacted. In terms of the organisational level specifically, stakeholder relations, business operations and economic stability is constantly adapting, often dependent on the stage in the life cycle. Additionally, SMEs do appear to be defined by relationships with other stakeholders, this was evident in the form of affective power of stakeholders with personal links, business power as a large customer or supplier for example, or relational power in embedded or socially proximate communities.

Society is ultimately just webs of relations among people and therefore the moral development of society is dependent on the ethics of human actors and nature of such business relationships. The scope of SMEs social engagement is therefore highly dependent on individual actors and webs of relationships influencing and working in parallel. There was a spectrum of instances whereby moral proximity was notably impacting decisions on CSR, yet the influencing power of proximity was infused throughout the findings and will be examined in more detail in the discussion. 
This section concludes the findings and analysis chapters. The proceeding chapter will discuss these findings in relation to the current literature and theories drawn upon to direct this research, as evaluated within the literature review. 


\section{Chapter Eight: Discussion}

\section{1: Introduction}

There are a number of ways that this discussion chapter could be structured, all with potential advantages. However, to build on the findings and analysis in Chapters Five to Seven and to ensure clarity, the following will be structured in line with the five key research objectives. Significant themes that have been configured during the discussion will then be refined in the concluding comments.

The first section will relate to the more general findings of the research context, CSR in SMEs. This will refer to the definitional ambiguity and unique conceptualisation of responsibilities for SMEs. The results will be compared and contrasted against the expanding, but still incomplete understanding on the concept, followed by an evaluation of the appropriateness of Spence's (2016) redrawn SBSR pyramids. Subsequent to this, the second section will look at the motivations of CSR, attempting to determine what drives SME engagement and if their perspective is broadly moral or motivated as an economically rational concept.

The third section will focus more specifically on the complex role and dynamics of stakeholder relationships, establishing the appropriateness of the ethic of care as a moral perspective to explain how this influences CSR in SMEs. Following this, the fourth section will explore the role of moral proximity as a space based analysis for CSR, to open up conversations regarding context and business structures that might impact decisions.

Finally, the perceived and potential social impact of CSR in SMEs will be considered. The difficulty of measurability and dissemination of information will be touched on, but the majority will centre on fundamental discussions related to the value of CSR and need for responsible CSR behaviour. These five themes will then inform the concluding chapter to follow, where the main question of how CSR is constructed and practised in SMEs and the role of moral perspectives to explain such engagement will be addressed. 


\section{2: Corporate Social Responsibility (CSR) in Small and Medium- Sized Enterprises (SMEs)}

This subsection of the discussion will focus on how the findings of this research relate to the current literature of CSR in SMEs. This will look more specifically at the important role of terminology to describe, but also uncover the true nature of CSR in SMEs, referring to Baden and Harwood's (2013) critical exploration of CSR terms. The unique conception of CSR in the findings will further be considered in relation to Carroll's (1979) four-part model, Baden's (2016) reconstruction of Carroll's pyramid and Spence's (2016) redrawn SBSR pyramids. This will address RQ1 and RQ2 of how SME owner-managers make sense of their responsibilities and role in society and the relevance of the ethic of care.

\subsection{1: CSR Terminology}

It is claimed by Baden and Harwood (2013) that the current CSR terms used to address CSR in SMEs, are deemed not fit for purpose. Findings of this research project support these claims and reveal a definitional ambiguity for SMEs when referring to socially responsible initiatives (Garriga \& Melé, 2004; Windsor, 2006). The ambiguity evident in the findings is in line with the view that CSR terms adopted via economic theory are 'jargon' (Baden \& Harwood, 2013, p. 615) and either not understood by SMEs or cause active disengagement to the terminology because of connotations antithetical to their ethical pursuits.

The first finding to take from the research is disengagement with the CSR acronym. Thirteen out of thirty owner-managers in the interviews had no awareness of the term and its meaning. Engagement with initiatives considered CSR were commonly described; yet not called anything. Responsible initiatives were simply considered the right thing to do for those respondents, integrated into business operations. Such silent or sunken CSR is a common characteristic of SMEs first coined by Jenkins (2004) and more recently by the likes of Perrini (2006) and Jamali et al. (2009), acknowledging that often unknowingly, businesses engage with CSR. This 
prevalence of silent CSR in the findings pertains to the ethical perspective, with SMEs having limited expectations of economically beneficial outcomes.

The disengagement of the term was also due to a lack of identification with the word 'corporate', considering it as excluding for SMEs as a collective. There are two reasons for this, the first due to SMEs not classifying themselves as corporate businesses, and the second due to connotations towards the large corporate conception of the term. Owner-managers perceived that CSR for large corporates was a balance sheet task, used as marketing for "corporate self preservation" (Wickert et al., 2016, p. 1170) that does not reflect the actual CSR being practised. This misalignment and perception of greenwashing or pseudo CSR has resulted in intentional disassociation with the term. This is in order to position CSR efforts as driven by more ethically sound motives and as to preserve legitimacy of SME activities and the social identity of the owner-manager.

For SMEs in this research, the findings were opposite to those of large corporates. They show lineage to what Wickert et al. (2016) described as a mismatch of symbolic and substantive CSR, outlining a communication gap for SMEs whereby their emphasis is on implementation rather than communication. This is limiting for the diffusion of CSR terms amongst SMEs, although arguably, due to the negative connotations and entrapment of the business case logic for CSR language (Brooks, 2010), this may not be a bad thing. However, the lack of communication does stagnate dissemination of knowledge between SMEs and deters adoption of one unified CSR term that more suitably reflects SME's unique engagement.

Due to the ambiguity discussed above, SMEs adopted a diverse spectrum of language to describe their CSR endeavours, with 'CSR' acting as a "metalanguage" (Murillo \& Lozano, 2006, p. 228). This can be an issue as it leaves scope for businesses to define CSR around their own self-interest, sometimes used outside its intended meaning (Baden \& Harwood, 2013). The focus groups alone revealed the adoption of twenty-one different CSR terms, with an additional six identified in the interviews. 
Terminology used by SMEs makes initial indications of how CSR is made sense of, locating the concept in this instance with reference to intrinsic moral awareness, philanthropy, community engagement and universal principles. Terms adopted were usually personalised around the SME's CSR interests or adopted from exposure via key stakeholders such as large customers or government. Two key points were first, the continued and persistent referral to community, despite the changing dynamics of the business-community relationship and second, the overtly moral language being used as alternatives to CSR.

Whilst awareness of the CSR term does seem to be increasing due to exposure from key stakeholders, still only two of thirty interview respondents adopted the term within their SME. On both occasions this was due to a desire to stay synonymous with key stakeholders such as large business customers. It was common that ownermanagers acknowledged what the concept encompassed, yet were reluctant to adopt, suggesting that there is a need to progressively replace or adapt the term to be closer to SMEs reality (Murillo \& Lozano, 2006).

This research indicates the need for a term that maintains consistency, but distinguishes from the large corporate conception of the term. Despite derivatives of community being dominant for SMEs, there is a need for a term that encompasses social, environmental and economic sustainability for society as a whole. The dynamics of community are changing and the implications on community engagement are currently unknown. Consequently, it seems obvious that the common rise of the term 'social responsibility' or 'small business social responsibility' is justified. The challenge is to disseminate these terms, whilst ensuring that the morally grounded CSR conception of the term is maintained.

To summarise, the intention of this conversation is not to release another CSR term to the debate, but to consider CSR terminology in relation to the findings. Language creates our reality and can command our belief systems (Baden \& Harwood, 2013) this can uncover truths and locate the context in which the concept of CSR is socially constructed. Definitional ambiguity is a persistent issue deterring adoption of CSR language in SMEs and creating confusion, despite increasing awareness of the concept. However, SMEs disassociated with the large firm conception of the term 
due to the co-opting of a moral concept by economic rationality. Buchholz (1991) says, "we must challenge those who neglect an ethical ought in CSR whenever a technical must (economic rationality) takes precedent” (p. 22).

Closer scrutiny of the language adopted by SMEs for socially responsible initiatives revealed their conception as overtly moral or community centred. Considerations from these findings imply the need to encourage SMEs to communicate their perception of social responsibility. By promoting the dissemination of moral language from SMEs in particular, the infiltration up to larger corporates could initiate a renewed focus on the ethical origins of CSR and stop the colonisation of economic rationality in the SME conception. It would also make visible the true value of SME's contribution towards society.

It is key that the power of business language is acknowledged and approached with more caution in the future (Murillo \& Lozano, 2006). As Baden and Harwood (2013) said, "our ideas are dependent upon the words we use to describe them" and "the terminology we use influences how our world is perceived" (p. 621). They share similar thoughts with Wicks et al. (1994) who considers it "essential that we remain continually aware of the dominant metaphors we use to describe ourselves and our practises because they often shape us in ways that we don't initially recognize or desire" (p. 475). By describing businesses as purely economic entities, it is not only describing a reality, but it "norms and justifies it, making it more 'true"" (Baden, 2016, p. 11), what can be described as a double hermeneutic (Giddens, 1987). The dissemination of moral language is therefore needed in order to recreate CSR as a moral concept and normalise this as reality within society.

\subsection{2: CSR Conceptualisation}

Having discussed CSR terminology, this section considers the unique conceptualisation of CSR from an SME perspective. The following will specifically focus on Spence's (2016) work to review the SME conception. Her redrawn categories of responsibility will be used to reflect on how respondents made sense of and executed their CSR. However, this is not to comment on the perceived fulfilment of each domain, as this is outside the boundaries of this research. 


\subsubsection{1: Survival}

The results from the interviews revealed one explicit occasion whereby an ownermanager followed Friedman's assertions that "the social responsibility of business is to make a profit" (1970, p. 13). However, even on this occasion, CSR engagement later identified for this SME was altruistic. More commonly, SMEs were content with pursuing a stable level of operations to minimally ensure business survival. Profit satisficing was evident with the economic responsibility considered as having enough to 'get by' in order to 'survive' (Baumann-Pauly et al., 2013; Spence \& Rutherfoord, 2001; Spence, 2016). In terms of the prioritisation of responsibilities, respondents displayed two perspectives; (1) CSR was engaged with as long as it did not do 'too' much harm to the business (2) CSR is something that is embedded within the business emanating from the morals of individual actors.

Respondents acknowledged their reliance on other stakeholders to fulfil their economic responsibilities. Most identified with a long-term perspective, viewing their responsibility to survive or grow as vital to maintain employment for themselves and others and sustainable, quality service in line with owner-manager expectations. This was not to 'create a quick buck' or 'get rich quick' for their own self-interests. Contrasts can be made to Carroll (1991) who emphasised the importance of being "committed to being as profitable as possible" with other responsibilities being "predicated upon the economic responsibility of the firm" (pp. 40-41).

Furthermore, Spence's (2016) redrawn responsibility of survival is dependent on having a license to operate and relies on adherence to minimum legal standards. Yet the findings of the interviews found that compliance of laws was largely absent from testimony. This does not suggest that there was no awareness, but perhaps that compliance was overlooked as an obvious requirement for business survival and social responsibility. However, there were occasions whereby adherence to health and safety laws or paying minimum wage were considered a contribution to the SME's social responsibility, not simply a legal necessity. 
Moreover, businesses are expected to adhere to the law as a codification of what society views as acceptable behaviour, yet knowledge gaps, insufficient resources or the perception that the SME size has limited impact can reduce the priority of adherence and result in amoral leadership (Carroll, 1991). For example, the noncompliance of laws in the informal economy was resulting in unfair competition for one of the respondents. This supports literature that has identified full legal compliance as unlikely if SMEs have insufficient knowledge and resources (Jenkins, 2006; Spence, 2016).

From the above discussion, it does appear that Spence (2016) is correct to claim survival as a suitable alternative for the economic and legal realm. However, whilst survival better acknowledges the fragility of the SME organisational circumstance, this research argues that despite the apparent appropriateness, there are still flaws in the structure. The responsibility of survival still suggests there are minimum profits that should be pursued in order to keep the company in business, regardless of the social demands (Kang, 1995). The freedom to subsume morality to economic pursuit implies that social responsibilies are only adhered to if the SME has margin to do so.

The above cognition is a flaw for SME owner-managers. There is a need to break away from the traditional Friedman paradigm into a new construct. The task that society faces is to gradually transform the conception of CSR from the first notion (1) CSR was engaged with as long as it did not do 'too' much harm to the business, to the second (2) CSR is something that is embedded within the business emanating from the morals of individual actors. Whilst it was evident that there was commonly a minimal economic pursuit before CSR was engaged with in SMEs, phrases such as 'I don't rate money highly' and 'I think there are more important things to life' imply that there were ulterior motives. There was also evidence that ownermanagers conceptualised their economic responsibility as one interlinked with that of key stakeholders, with their success dependent on and facilitating that of others. The pursuit of survival was therefore not only for their self-interest, but acknowledged as a role in the success of others.

To summarise, the empirical findings do support the alteration of survival to better represent the reality of SMEs. There was a dominance of profit satisficing as a more 
appropriate target for SMEs. In order to be economically viable, the organisation also needed to adhere to the law in so much as their knowledge and resources allowed. This then enabled them to operate in society, what we might consider as the 'iron law of responsibility' (Davis, 1960). However, SMEs cannot be seen as purely economic entities, detached from the individual (Sachs \& Ruehle, 2009). The freedom to prioritise economic survival over the personal integrity of ownermanagers should therefore be considered, particularly when CSR should be an inherently moral concept.

\subsubsection{2: Ethic of Care}

As discussed above, there were some instances whereby owner-managers described a perceived trade off between CSR and economic success. However, many respondents were of the impression that ethical responsibilities and CSR are synonymous and should be done irrespective of cost. This suggests that SMEs often prioritise ethical responsibilities before those of the economic. Whilst Carroll (2016) has recently emphasised that ethics permeate his CSR pyramid, the structuring of ethics after economic responsibilities still creates tensions because, when economic and ethical responsibilities are in interplay, one is usually at the expense of another (Aupperle et al., 1985).

With regards to the conceptualisation of ethical responsibilities, respondents consistently referred to a sense of 'duty' as their motivation for CSR engagement, following tenets of Kant's duty based ethics. However, the duty referred to was not commonly towards society in general, but to their specific physical or social communities of which the SME was embedded. Findings further emphasised the interconnected nature of SMEs, with close and multiple associations connecting the realities of stakeholders (Wickert et al., 2016). Caring beyond the societal standards towards stakeholders is not considered an ethical expectation of businesses, yet consistently the norm for owner-managers within both language and behaviour implied close relationships, with one SME contributing their whole business pursuit as being that of caring for individuals. 
This suggests that SMEs cannot be seen as detached from the human actors within (Jenkins, 2004; Spence, 2007; Spence, 2016), with decisions on CSR not only dependent on the expectations of society, but the individual realities of human actors (Kang \& Wood, 1995). The responsibility of owner-managers to make ethical choices is therefore the most fundamental of the CSR components for SMEs and influenced by key relationships and information they have as a decision maker with regards to the moral issue. The ethic of care accommodates this contextual dependency, suggesting that SMEs are bound by circumstance and relationships, whereby "the organization is run sensitive to human needs, not just as an economic production unit" (Spence, 2014, p. 384).

Owner-managers also consistently evidenced partiality towards those with close physical or relational ties. This hints at elements of the ethic of care whereby at the forefront of moral and ethical concerns is the additional responsibility that the business has towards dependents of the business (Spence, 2014). This does mean that stakeholders with close relationships to the owner-manager, or multiple relational ties are likely to have a higher moral claim, conflicting with the universal judgement of Kant (see for example 1781/1998; 1788/1997).

However, the ethic of care as a moral perspective does not discount universal ethics. Despite claiming partiality towards dependents of the business, it does not reject the acknowledgement of behaving ethically towards society as a whole, but simply that moral claims are intensified with personal links and relationships. CSR may be societal wide, but for SMEs it is enacted through more localised networks and communities. This is one of the many reasons why proximity has such important implications for CSR SMEs, as will be discussed in more detail in section 8.5.

This caring SME perspective could be important for the future of business. It is assumed by Carroll (1991) that we can only be ethically responsible if laws are effectively enforced. Arguably, it is ethical consideration that result in the codification of laws, in line with societal expectations. SMEs are often unaware if they are adhering to laws or not, particularly when constrained by a lack of knowledge or resources. In this case they are reliant on the ethical character of their business to anticipate and evaluate societal expectations, beyond those of legal. This 
is pertinent for the future due to increasingly niche and advanced technological developments whereby laws may lag behind. If SMEs are already more reliant on adhering to their ethical responsibilities, then this lag with codification of laws will not have negative implications for society.

The partiality towards those whom the owner-manager has a relationship could raise questions as to the ethicality of this moral perspective (von Weltzien Hoivik \& Melé, 2009). However, the personalised and interconnected nature of SMEs identified in the findings appears to intensify SME's perceived ethical responsibility. This was more than preventing ethical compromise, but evidencing behaviour that went beyond mere compliance of laws and societal norms, redefining their perceived ethical responsibilities as owner-managers. As SMEs are uniquely characterised by their ethical conceptualisation, it seems that the ethic of care does better reflect the ethical character of CSR in SMEs.

\subsubsection{3: Philanthropy}

For many respondents, CSR was confined to the philanthropic domain. 'Charity', 'donating' and 'giving back' were commonly depicted as their understanding of the concept. This is not surprising considering that the charity principle has been evident for hundreds of years (Brooks, 2010) and underlies the concept. There was one occasion whereby the difference between ethical and philanthropic responsibilities was distinctly articulated, with ethical relating to existing contractual relationships and philanthropy that can occur where there is not. Interestingly, respondent P18 describes philanthropy as the last responsibility prioritised, not because it is not important, but because of the belief that you cannot be philanthropic with someone else's money when it is for personal interests. His view was that there is a formal and therefore ethical responsibility towards those with a contractual relationship, of which he interestingly included family (Spence, 2016). These stakeholders are prioritised before those to whom there was no formal responsibility and are the differences used by Spence (2016) to justify the continued separation between ethical and philanthropic responsibilities. 
This follows similar thinking from Kang (1995) who proposed the concept of corporate social benevolence (CSB). There are numerous discussions identifying charity with CSR (Brooks, 2010), criticising the identification of personal charitable donations as CSR (Friedman, 1970), or indeed arguing that voluntary philanthropy unrelated to the firm's social involvement could be seen as benevolent to the business. Kang and Wood (1995) contend that it is selfishness from the ownermanager when CSR is based on the 'personal drivers' or charitable preferences of the individual, as opposed to the SME.

However, philanthropy in the findings was often a by-product of personal experience of the owner-manager or dependents of the business. There was evidence of the inception of charitable requests filtering up from employees or collaborative efforts with key stakeholders. This aligns with the ethic of care perspective that claims naturally, individuals will show partiality towards actors and causes with personal significance (Spence, 2007). This is linked directly to proximity and will be discussed in section 8.5. In defence against the CSB claims, arguably entities cannot be separated from the individual actors and the values the business was built upon. Owner-managers are often the key financial investors of the SME and therefore can the pursuit of personally driven philanthropy still be considered as selfish? If ownermanagers did attempt to separate the personal from business, there is the risk of making decisions within a moral vacuum; easily losing sight of the human aspect of morality that should be the foundation of CSR (Kang \& Wood, 1995).

Concerns were raised by P15 that 'only businesses in good, high performing economies can form the voluntary precept'. However, the preference to donate time and skills was common (Wickert et al., 2016), utilising resources and overcoming this perception. SMEs perceived this form of philanthropy as the most valuable in terms of their investment and perceived impact for recipients. By engaging with philanthropic activities in parallel with business competencies, it allows philanthropic engagement without necessarily investing financial capital, simply time as a cost. Personal time investment and knowledge was considered as more valuable assistance that can result in a larger impact on recipients that are in need of support. Kang and Wood (1995) argue that providing that philanthropy is justifiable within the firm's social involvement, it can be considered CSR or 'charity-within- 
CSR'. It could also be argued that within SMEs, philanthropy aligned with personally significant preferences should not be considered as CSB due to their personal investment and altruistic motivations.

Finally, philanthropy and charitable giving can be traditionally linked to religion (Brooks, 2010; Spence \& Schmidpeter, 2003), yet it was largely absent from testimony in this research. The findings implied that there is an increase in secularism in the UK society and consequent changes to the nature of philanthropy and CSR. The recent rise of social enterprises and community interest companies (CIC) could be seen as a representation of these changes, or even a gradual replacement of the strong religious foundations of charity as our society diversifies.

\subsubsection{4: Personal Integrity}

Findings undoubtedly emphasised the importance of individual level responsibilities. CSR engagement was inextricably linked to personal values, with respondents stating that it should be an embedded principle in everything owner-managers do and the starting point of business. This means that business and ultimately CSR would be an extension of the owner-manager's moral consciousness. Respondents explained their responsible behaviour as something that is engaged with because they 'know it is right', arguing that they 'don't have to be taught, it is coming from personal values'.

Values and personal integrity in practice should be flexible and sense-making constantly adapted (Fevre, 2000), the findings relate to this claiming that ownermanagers of SMEs can be more responsive to CSR because they are doing it as 'socially responsible people' and not having to deal with the 'bureaucracy of a large organisations'. However, personal integrity by its very nature is diverse and subjective to the individual's moral framework (MacIntyre, 1985). What is considered as a moral act is drawn from their internal value system and constructed within their reality, of which is embedded in social structures.

Honesty, authenticity and reputation are elements of social capital (Sen \& Cowley, 2013) described by owner-managers as important during the data collection. These 
are all encompassed in the personal integrity domain introduced into Spence's SBSR pyramids. According to Jones (1991), personal integrity requires owner-managers to draw from an internally consistent moral character, emphasising honesty and authenticity and considering the degree of importance of each act in order to come to an ethical decision. Individual decision-makers have the freedom within organisations to exercise choice; therefore it is their obligation to "exercise such discretion as is available to them toward socially responsible outcomes" (Wood, 1991, p. 696).

However, one prevalent finding was the perceived dishonesty of large businesses and their CSR claims. There was a perception that large businesses cherry pick the metrics that best tell the story for their stakeholders. One owner-manager also considered a misalignment with the motivations and engagement with CSR, whereby businesses are doing the right thing for the wrong reason (Wickert et al., 2016). As Killinger (2010) comments, "there is no integrity in saying one thing and doing another" (p. 12). This perception of CSR has led to a disassociation with the term for SMEs. This disconnection can be seen as a preservation of the owner-managers personal integrity. It also aligns with the view that SMEs are inherently more intrinsically socially engaged in comparison to their larger counterparts.

A further point of discussion is the intrinsic engagement of owner-managers influencing CSR on an organisational level (Corbin \& Strauss, 2008; Spence, 2014; Spence, 2016; Wood, 1991). There are a number of examples from the findings that evidence this. The first is the synonymy of the reputation of the SME with the reputation of the owner-manager. This characteristic of SMEs means that there is more at risk for the individual's reputation if the business does not adhere to ethical expectations of the community. A further example is the pursuit of stakeholder relationships based on the alignment with personal values of the owner-managers. Moral alignment of stakeholders can be extremely beneficial in terms of mutually accepted values and unifying the ethical character of the SME, it was also considered as a necessity for long-term stability of SMEs.

Ethical decision-making always comes back to the individual, with personal integrity evidently influencing CSR engagement in SMEs. It seems obvious therefore that the 
morality of individuals should be the basis of reasoning for all conversations regarding CSR, confirming the appropriateness of the ethic of care approach.

\subsection{3: Spence's SBSR Pyramid Structure}

Having discussed the relevance of the individual components, attention turns to the structure and applicability of Spence's (2016) redrawn SBSR pyramids. The aim of this research was not addressed through grounded theory or intended to propose drastically new models, but to establish the relevance of moral perspectives to better explain CSR in SMEs. The relevance of the redrawn domains was broadly supported by the findings. However, the radical departure of structure from Carroll's pyramid to accommodate the specific and contextualised aspect of SMEs did create some confusion when discussed with participants. A number of considerations will be outlined in the following.

The first consideration is the disaggregation of the pyramids to align with key relationships. Owner-managers, family, business partners and local community are key stakeholder relationships, but the non-homogenous nature of SMEs means that these do diversify dependent on context. Allowance for that would be welcomed. For example, the perception of what constitutes the SME's community could create complications, as discussed in section 8.5.3. Furthermore, it could be limiting to cluster suppliers, customers and competitors under business partners. Each of these stakeholder groups has distinct needs and salience is dependent on a number of complex factors. Consequently, despite the redrawn pyramids being a welcome development, the appropriateness to group or limit stakeholders to prescribed categories of each SME could be questioned.

Having discussed the move from a general CSR pyramid to the more specific SBSR pyramids, attention turns to the ordering of the domains. Spence (2016) contends that this should follow the perspective of the owner-manager and the domains ordered according to the desirable and essential responsibilities towards the stakeholder in question. Yet, when discussed with participants, SME ownermanagers found it difficult to comprehend whose perspective they should be taking, that of themselves, the SME, or the stakeholders. This may stem from the merged 
personal and business spheres of SMEs and inability to distinguish between different roles and corresponding responsibilities. This confusion meant that the findings were not easily comparable, evidencing the complexity that this research has uncovered.

Finally, this research supports and shows findings aligned with the redrawn domains. However, when reflecting on the ordering there are a few key considerations. The responsibility of survival still implies that there are "minimum profits that should be assumed in order to keep the company in business" regardless of social demands (Kang, 1995, p. 7). This contention is one that is grounded in the early neoclassical assumptions of economic rationality. The salience of the economic is still therefore present, creating tensions with CSR as a moral topic in that as long as there is an economic pursuit, be that maximising or satisficing, this will supersede the moral.

It is proposed that the moral responsibilities of the owner-manager to make ethical choices are the most basic and compulsory components of CSR on which all others should rest and therefore personal integrity, encompassing such morality, should be an essential domain at the base of each pyramid. As argued through this research, the ethicality of a business allows the SME to operate in their community and society as a rule. Therefore, sustainable economic success may be more likely if it follows a moral grounding.

A further tension identified in the new model relates to the omission of legal as a single responsibility. Whilst the survival component was deemed as relevant for SMEs, there may be tensions with the new model because ethical domains are in constant interplay with legal, re-creating and adjusting to new societal standards. As Fevre (2000) explains, we have no absolute reality, just a relative one. This interplay is less obvious when re-created as survival and is something that should be considered.

\section{3: Key drivers of CSR in SMEs}


The following discussion will address RQ3, in an attempt to establish the key drivers of CSR for SMEs. To organise this discussion, the CSR perspectives proposed by Wickert et al. (2016) will be adopted. This is because they are simple categorisations of the three most common motives drawn from existing literature. Drivers of the SME were derived from interaction with owner-managers due to their decisive role in the overall perspective adopted by the SME. The main aim of this research is to broadly establish if CSR is constructed and practiced as a moral concept or otherwise. Finally, before distinguishing between perspectives, it is important to note that these are not mutually exclusive and different perspectives can occur simultaneously (Aguilera et al., 2007).

Figure 8.1: CSR Perspectives (Wickert et al., 2016)

Economic

Relational/

Ethical

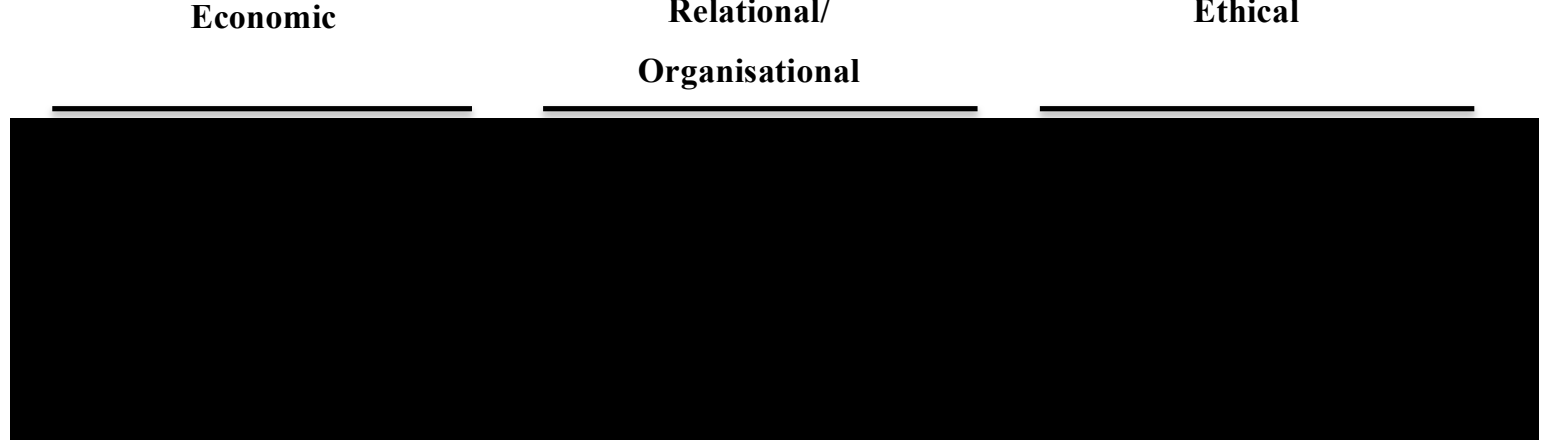

activities

\subsection{1: Economic Perspective}

Drivers explicitly associated with the pursuit of short-term profit were not common, although the contention that CSR engagement was predicated on some sort of optimum amount of profit was apparent for a number of respondents. This follows Friedman's (1970) paradigm whereby economic responsibilities are prioritised before moral. One example of this is in section 6.5.2 whereby an owner-manager comments on the need to give back but adds, 'without hurting the business', referring to the financial position of the SME. They further consider that if in addition it actually helps or adds to the business in some way, then that is a bonus. 
This still implies some sort of trade off between CSR engagement and economic success for the business.

On no occasion did owner-managers imply an economic or instrumental perspective of CSR engagement for direct personal gain (Aguilera et al., 2007). On one occasion of planned, voluntary CSR (Torugsa et al., 2013) a respondent described significant increase in profits following charitable events, describing it as a 'win-win' situation. Following this there were CSR efforts that could be described as proactive, such as apprenticeships that reduce staffing numbers and costs, or on a reactive basis whereby quality marks were pursued as a requirement for tenders to ensure competitiveness for the contract.

A further example of reactive CSR with an instrumental perspective was engagement to ensure a tax break, this was not for the SME participant but was an example provided from their SME clients. However, despite instrumental perspectives such as the above, there was consistent referral to these activities as being mutually beneficial for both recipient and SME. It could be considered then, that on many of these occasions, despite motivations being of an instrumental nature that there is still perceived value in their CSR engagement for recipients and contribution to wider societal wellbeing. Although, as discussed in 8.2.2.5, this could be considered as doing the right thing for the wrong reason (Wickert et al., 2016), bearing in mind that CSR should be a moral concept (Baden \& Harwood, 2013). Integrity of the individual actor and SME could therefore be questioned (Killinger, 2010).

\subsection{2: Relational/Organisational Perspective}

The second perspective proposed by Wickert et al. (2016) is the relational or institutional. Motivations for CSR related to this perspective were common in the findings, with there were three common forms of CSR engagement. The first includes instances whereby SMEs were approached for their expertise via stakeholders; this was reacting or waiting to adhere to external demands. One example was as a consultant to assist the development of a business plan for new sports facilities in the community and another to formally risk assess the development of a sewage works. In the latter case, the community ended up with a 
solar farm rather than a sewage site, the value of this contribution for the community and environment was significant in this case. P7 contended that they always adhere to CSR requests, but do not have time to be proactive if not presented with the opportunity, what is considered strategic or opportunistic.

The second form of engagement was SMEs actively engaging with CSR due to the expectation of positive relational implications for the SME. This could be considered as a strategic approach to CSR, although the word strategic should be used with caution as it has been used synonymously with instrumental on occasions (Lantos, 2001). For example, respondents of the focus groups (see section 5.5) and interviews considered reputation, trust, networking and word of mouth as key benefits sought from such engagement. These all could be considered as aspects of social capital (Fuller \& Tian, 2006). For example, P24 considered the development and maintenance of reputation to be a key motivator for CSR and P10 motivated by positive word of mouth as the 'best kind of recommendation'. P22 was also motivated by the networking opportunities and 'speaking to people'.

From this we might contend that $\mathrm{P} 10$ is driven by relational factors closer to the instrumental side, as it is for public relations and competitive advantage. However, in further comments P22 refers to their SME as a social business, with social responsibility not considered CSR, but an outcome of being decent people and collaborating with the community. Bourdieu (1986) considers that networks of relationships offer members "collectively owned capital, a 'credential' which entitles them to credit, in various senses of the word" (p. 252). Similarly, the sense of reciprocity valued by $\mathrm{P} 10$ was not considered a commercial trade, but an organic process whereby 'emotional credit may be used the other way around'. This aligns with Bourdieu above and Polanyi's (1944/2001) 'performance of acts of exchange', whereby acts are reciprocated, but "not necessarily by the same individual" (p. 49). This infers similar characteristics of social capital. P10 appears to have presented a paradoxical account of their SME drivers of CSR. This was not uncommon during this research and emphasises the complex, reciprocal and dynamic nature of motivations. 
The third and final form was whereby SMEs engaged with CSR, but the beneficial outcomes that occurred for the organisation were not sought or expected. These could be considered as 'indirect benefits'. As P7 explained, 'I don't think we expect anything back from it but we just think it does have an effect in terms of our business'. Respondents engaging with this form of CSR considered it as a duty, 'without any strings attached'. There was a perception that any positive reciprocation for the business is 'a bonus' but 'not the be all and end all'. It was acknowledged that when this perspective is adopted, often the SME gives more than what they get back from it. This perspective is weighted towards the ethical, as the benefits for the SME are considered as indirect and unexpected.

For MacIntyre (1985), morality has lost meaning because there is a reliance on reason as opposed to belief. The first two forms of CSR seen in the relational/organisational perspective could be considered as reliant on reason, whereby CSR is motivated when a positive organisational reason for engagement is presented via external or internal stakeholders. The third form however appears to be reliant on belief via the owner-managers internal moral framework, whereby CSR is motivated by the belief that it is the responsible action to take, with the organisational benefits defined and justified in retrospect.

The legitimacy of CSR activities and drivers are therefore complicated, particularly for the relational/organisational perspective positioned between the economic and ethical. The dynamic nature of motivations and lack of understanding of their interaction complicates the categorisation of CSR drivers, whereby it may move dependent on differing factors. For example, expectations of society and the legitimacy of activities are constantly being readapted and therefore expectation of stakeholders will not remain consistent. As Fevre (2000) explained "we have no absolute position, just a relative one that we must continually re-establish" (p. 8).

\subsection{3: Ethical Perspective}

The third and final perspective is the ethical. The findings of this research found that it was the ethical perspective most commonly referred to when considering the motivations to participate in CSR activities for SMEs. The view that businesses 
'shouldn't be doing it for profit' or financial gain was frequented in the findings and altruistic tendencies were observed on numerous occasions, whereby reciprocation was not expected. As P10 explained, 'it's not a sort of tit for tat'. P12 further supports the non-economic focus when explaining 'I don't do it so I can get money and get people, I do it because I like to give a little bit back'.

Within this perspective, respondents often referred to the intrinsic motivations of the owner-manager. They appeared to consider these motivations as interrelated with that of the CSR drivers of the SME. This aligns with Spence's (2016) view that owner-managers are central to the stakeholder map of SMEs and largely influence organisational practise. On one occasion, the driver for CSR was considered to be 'love' and another as simply 'humanity'. Love could be considered as going beyond morality because it is a natural human impulse, not constricted to a socially constructed set of moral rules. However, most commonly it was a moral obligation to do the 'right thing' or a perceived sense of 'duty' to give back that was the driver behind CSR engagement.

P2 amongst others claimed to be driven by 'the right thing, what's fair and right'. He considers that the SMEs goes 'with what's the right thing to do and not, not what's the financially best thing to do'. This is an ethical perspective of CSR, although caution should be exercised when talking of the 'right' act. As discussed earlier, for some the right act for a business would be to pursue economic maximisation (Friedman, 1970). Therefore, by the SME not choosing the financially best option, it could be seen as an illegitimate business activity. Furthermore, what is right or wrong is also dependent on internal moral frameworks of decision-makers within the SME, socially constructed in line with cultural and social conditions they are embedded within.

The feeling of duty to give back was commonly driven by personal association to causes or a feeling of moral obligation towards those close to the SME or those in need of assistance. As P8 explained 'you naturally go for things which mean something to you'. Justifications for CSR were linked to embeddedness within communities. With reference to this, P2 said, "part of your success is due to your environment and where you are so it seems that the least that you can do is to give 
back'. There was a further sense of appreciation towards the environment that facilitates the success and position of the SME. This was justified by P15 as follows; 'because I'm in a position where I can do it I feels like I should' and 'I should be because I am part of the community'.

P5 further explains affection to his community by saying, 'I want to see this area great, so you know I don't mind putting my time in'. Although, when talking of an ethical perspective, morality should be inherent in everything the SME does, not only when situated in a certain position or locality that might elicit a sense of duty. This finding does relate to the ethic of care perspective, whereby it is contended that we are naturally inclined to care about those close to the individual or business, physically or emotionally (Jaffe \& Pasternak, 2006). This reveals the integral role of proximity and stakeholder dynamics, as will be discussed further in the following section.

Many owner-managers considered that as individuals, the intrinsic 'sense of satisfaction' and 'doing something worthwhile' when assisting the community or those in need was also a motivating factor. Again, this brings the CSR drivers back to the intrinsic emotions of individuals within the SME. For example, P18 explains 'it was actually quite satisfying to bring my actual day to day skill into the local community', 'it gave me a buzz'. For P27, it was knowing that he was helping vulnerable people to turn their lives around that drove his engagement, with the motivation described as 'seeing those changes'. It could be concluded then, that the self-satisfaction of assisting those in need, via either the contribution of resources or knowledge was a dominant driver for SMEs.

A final ethical driver for CSR was the accountability towards diminishing resources and the maintenance of the current environment for future generations. It was considered that businesses should hold themselves accountable for this, with one owner-manager envisioning a world whereby 'everyone does give something back'. For example, P6 explained their motivation to buy biodegradable coffee cups despite the cost implications, their justification being that 'we should probably be doing this for the sake of the environment'. Furthermore, P1 describes the motivation of CSR in her SME as being considerate of future generations, "when I have children I want 
them to be able to experience life as I've had it'. These examples show how SMEs were considerate of others, even those of the future, aligning with a new paradigm of thinking whereby it is "the moral and business principle to give each person, in each generation, the opportunity to prosper" (Roche \& Jakub, pp. 42-43). This is proposed not for individual gain, but for the greater good.

\subsection{4: Dynamic Perspective}

Despite the effort to classify CSR drivers into three perspectives, the results identify that these classifications are in interplay and change over time. Therefore, we might consider CSR perspectives as dynamic (Ackerman, 1973; Ackerman \& Bauer, 1976) and not mutually exclusive. There are continuing debates over the interaction between instrumental and morality based motives, with acknowledgement of relational motives falling somewhere in between, yet "no consensus to date on how such motives interact" (Aguilera et al., 2007, p. 843). What is agreed is that they are complex and can be seen to sit on a continuum. This finding might explain paradoxical accounts of CSR drivers from owner-managers. For example, when SMEs proactively pursue relational benefits of CSR for competitive advantage, this might be situated in the relational perspective, but interact closer to the economic rather than ethical.

Aguilera et al. (2007) refers to motives as "working simultaneously", with some "more salient than others" (p. 847). This describes the findings of the study more appropriately and opposes Windsor's view that economic and ethical viewpoints do not overlap conceptually. There are a number of reasons why the drivers of CSR might alter with time. One example from the findings refers to their perspective as emergent as the SME progressed through the business life cycle. P26 was first driven by CSR as a strategic activity for public relations, this later transferred to an ethical motivation to give back to personally relevant causes, showing movement on the continuum.

Literature in the past has suggested that the morality of managers operates on a continuum (Carroll, 1991) and that ethical orientations are non-static, shifting between frames (Spence \& Rutherfoord, 2001). However, these relate specifically to 
the motivations of the owner-managers. Whilst the findings of this research accentuate the role of the owner-manager, it outlines the drivers of CSR for the SME as a whole. Polanyi's (1944/2001) perspective is of value for this finding as it acknowledges context as a dependent variable for CSR. This is relevant to accommodate external factors that influence the CSR perspectives.

The reference to influencing factors above brings about the question of the typology of CSR, considered to range between proactive and reactive (Torugsa et al., 2013). P25 claimed that SMEs are able to be responsive, as they are 'not dealing with the bureaucracy of a large organisation'. However, the findings suggest that whilst SMEs are more responsive to CSR opportunities presented by stakeholders, this is not perceived as a managerial move in response to threats. Furthermore, whilst examples of proactive CSR were limited in comparison, there were examples whereby SMEs pursued relational benefits of CSR such as networking opportunities, publicity or word of mouth. There was however still a lack of proactive CSR whereby it supported economic, social and environmental responsibilities sustainably.

\subsection{5: Summary}

To summarise, we cannot assume that owner-managers or key decision makers of SMEs are economically rational actors in pursuit of profit maximisation. In fact, there were very limited findings that suggested this. SMEs did often consider CSR to have costs for the business, but broadly speaking it was considered as something that should not be done for purely economic gain (Spence \& Rutherfoord, 2001). Although perspectives cannot be discerned with complete accuracy, there was basic agreement that SMEs were driven by ethical or relational/organisational motivations. Drivers for CSR are increasingly complex and influenced by intrinsic and contextual factors, on both a reactive and proactive basis. This resulted in a sense of perplexity and paradoxical accounts of drivers by owner-managers. However, on no occasion did any participant suggest that SMEs should not engage in CSR; in fact, most considered that it should be embedded in the structure of the SME. 


\section{4: Stakeholder Prioritisation and Relationships}

As is evident above, stakeholders are an influential driver for CSR in the context of SMEs. The following sub-section discusses the complex role of stakeholders and dynamics of relationships, referring to Freeman (1984), Freeman and Liedtka (1991) and Wicks et al. (1994) with their perspectives on the stakeholder approach. A further aim is to establish the appropriateness of the ethic of care as a moral perspective to explain CSR and webs of interactions in SMEs, referring specifically to Spence (2016) and her proposed 'Redrawn Stakeholder Theory'. The following will address RQ4 and RQ5 of how key stakeholders of SMEs and the nature of relationships influence CSR inception and engagement.

\subsection{1: A Different Generalised Set of Stakeholders}

The ethic of care perspective of stakeholder theory suggests that SMEs have a different generalised set of stakeholders, proposing that organisations are arenas of both public and private spheres (Spence, 2016). One of the main propositions of the redrawn theory is that at minimum, "the key stakeholders for small businesses are likely to be employees, small local competitors, suppliers, local community, family, and customers" (p. 35), with the owner-manager at the centre point.

However, there were limited examples in this research whereby owner-managers listed themselves as a key stakeholder. On one occasion an owner-manager did quote, 'without myself being well and without my family's support I couldn't do it' and another whereby they said 'it's just me, so yes of course I'm very important'. However, despite the lack of explicit comment, it was implied throughout the findings and discussed in section 8.2.2.5. Therefore, it could be assumed as an axiomatic truth, overlooked by owner-managers as something that is self-evident. This confirms Spence's (2016) proposition that owner-manager(s) are the centrality and legitimate decision-maker of the business (Fassin, 2008; Quinn, 1997). Although, this is not to suggest a dyadic, management-centered view, just their fundamental role as stakeholder for SMEs in this research. 
In terms of key stakeholders for SMEs in this study, it appears that Spence's redrawing is an over-simplification of the reality for SMEs. The findings of this research confirm that stakeholder theory is "multifaceted" (Jenkins, 2004, p. 44) and complex for SMEs. Stakeholders mapped and prioritised by SMEs went far beyond the simple classifications of both Freeman and Liedtka (1991) and Spence (2016). For example, financial institutions, CSO's (civil society organisations) such as NGO's (non-governmental organisations) and charities, local and wider government, education institutions, the media and business network facilitators were among additional stakeholders named and prioritised in the focus group findings.

Figure 8.2: SME Key Stakeholder Map

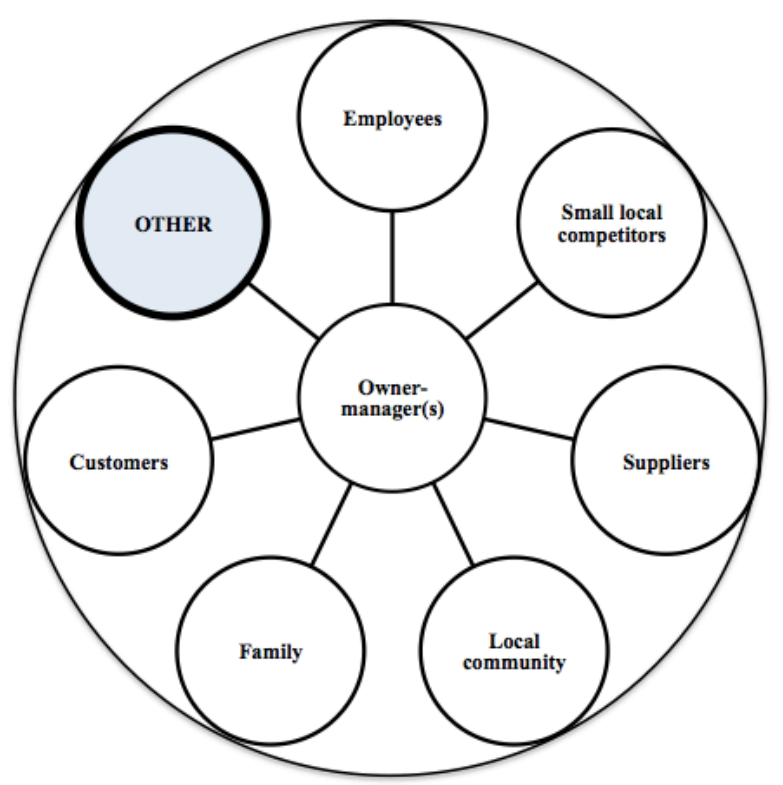

Figure 8.3: SME Key Stakeholders in 'Other' Category

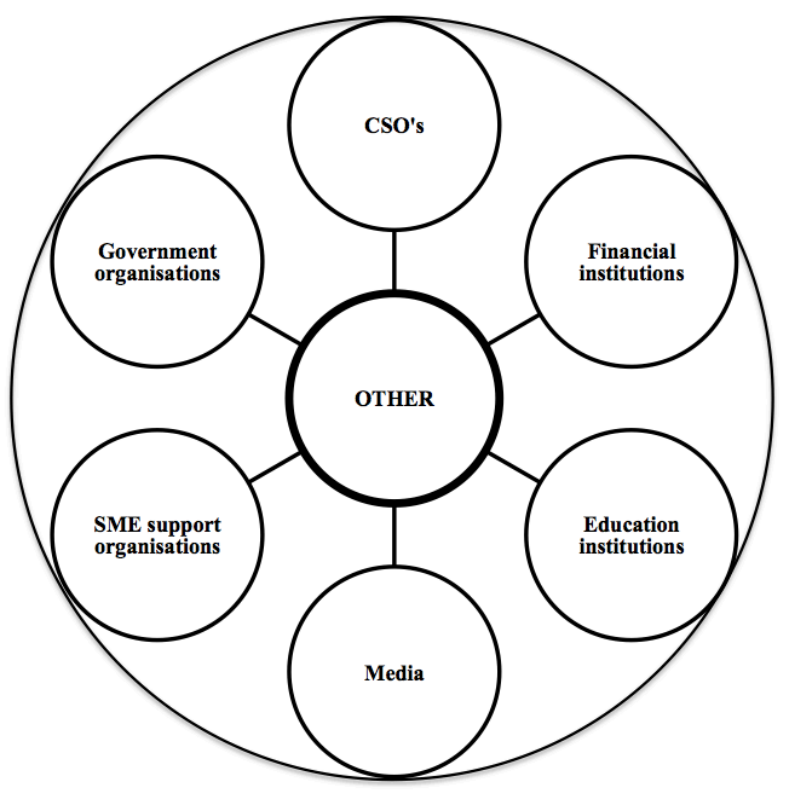


Additional to the variance of key stakeholders, there were further complicating factors. The first of these was the context specific nature of stakeholders identified. Names of individuals or specific businesses were used rather than stakeholder classifications. Often, the same classification was represented at different levels of priority, dependent on the specific person or business. It is acknowledged that in these instances, the researcher had the role to discern the classification. These findings reiterate that business is still about people, not "faceless groupings" (Spence, 2016, p. 34). Owner-managers made sense and prioritised based on their own social constructions of reality (Jenkins, 2006; Mitchell et al., 1997). This supports Polanyi's (1944/2001) contention that individuals and, importantly, their economic activity are submerged in social relationships and the concept of multiplexity (Lähdesmäki et al., 2017).

A second factor was the different levels within stakeholder classifications that alter their priority. This refers to attributes of the stakeholder salience literature (Lähdesmäki et al., 2017). One example identified was whereby employees were prioritised at different levels due to power differentials, such as senior management and support staff. The third factor was prioritisation of stakeholders being a reflection of the current SME circumstances. It was previously recognised that stakeholder salience is fluid and changing (Mitchell et al., 1997) and as P1 explained, stakeholders were prioritised on 'who is banging on the table loudest at any one time'. In reference to the literature, proximity highly influences salience and is of sufficient importance to merit a separate section of discussion.

\subsection{2: Spence's Redrawn Stakeholder Theory}

The relevance of the four enhanced elements of Spence's redrawn theory was covered above. However, it seems appropriate to outline key considerations with regards to the complexity of SME stakeholder networks. It was appreciated that Spence (2016) purposely simplified the categorisations of stakeholders, acknowledging "it is clearly not the intention of stakeholder theory to predetermine stakeholder groups", with the intention that "stakeholders should be calculated anew" (p. 28) for each business. However, it cannot be denied that the bilateral, onedimensional stakeholder map in the redrawing misrepresents the varied and context 
dependent nature of stakeholders integral to the ethic of care theoretically.

The simultaneous management of competing stakeholders was evidently difficult for SMEs. It is not surprising then that owner-managers found it challenging to discern which stakeholders were of most importance. Most often it was a closely interrelated web of stakeholders working in unison that enabled the business to operate, rather than individual stakeholders. Stakeholder theory has shifted to acknowledge "complex webs of relationships rather than just a series of dyadic connection between stakeholders and the corporation" (Russo \& Perrini, 2010, p. 209). However, the visualisation of Spence's redrawn stakeholder theory implies a simplistic connection between the SME and stakeholders.

Rather than display dyadic relationships between the owner-manager and key stakeholders, it is proposed that the reality of stakeholder theory for SMEs is better depicted via a system-orientated view (see appendix 8.1). This acknowledges a system of stakeholders that interact in order to pursue a number of goals and is similar to what Kang (1995) proposed, yet differs in that this research focuses specifically on SMEs and their key stakeholders. As Spence (2016) proposed herself, SMEs are defined by their role as a stakeholder of other organisations, rather than simply a central organisation it itself.

\subsection{3: The Nature of Stakeholder Relationships}

The emphasis on relationships for SMEs "makes stakeholder theory intuitively appealing" (Spence, 2016, p. 28). However, one of the main research questions (RQ5) was to assess the appropriateness of a renewed care perspective to better explain the nature of SME stakeholder relations (Spence, 2016; Wicks et al., 1994). This is in order to understand the impact of relations on the inception and engagement with CSR. It was obvious from the findings that SMEs are inextricably embedded in networks of relations. The nature of these will be discussed below.

\subsubsection{1: A Call for Care: Meeting the Needs of Others for Whom we Take Responsibility}


The call for acknowledgement of care within SME stakeholder relations (Spence, 2014, 2016) appears to be a relevant proposal. Examples of extracts from the interviews include the following; 'we're always caring, we're always looking after each other' referring to the community and 'we do care about them, they're literally like my children' in reference to staff. This caring nature of stakeholder relationships in SMEs was seen to transfer into CSR engagement, with 'genuinely caring for somebody' described as a CSR driver. This aligns with a key characterisation of the ethic of care perspective whereby strategy is "guided by sympathy, empathy, sensitivity, and partiality meeting the needs of others" (Spence, 2016, p. 33).

In line with this strategy, for SMEs it was often relational rather than hierarchical tasks that were prioritised (Spence, 2016). This was obvious in the findings whereby flat hierarchies elicited a transparent team atmosphere and sense of buy in. One respondent explained saying, 'it's all in, so everybody has to help each other out...if the tea needs making everyone helps make the cups of tea, if the phone's ringing we all have to pick the telephone up' and another with, 'nobody is above anybody else'. These structures of SMEs suggest that the caring approach is an integrated practice, aligned with Brooks' (2005) understanding of strategy. It also follows tenets of the feminist interpretation from Wicks et al. (1994) who views "strategy as solidarity" (p. 488) and promotes "the human capacity to engage situations with empathy and communication" (p. 488).

The unique, caring approach evident in relationships was considered a response to a sense of 'duty' from owner-managers. For example, when P18 said, 'I don't buy anything outside unless I have to, because I believe that people in this area give me my lifestyle'. A sense of obligation such as this can be associated with MacIntyre's (1985) discussion of honour, Weber (1930/2003) on 'the calling' and Polanyi (1944/2001) who considers that individuals have a place in society and therefore have duties. As identified above, the duty was towards stakeholders close to the business such as suppliers, employees and community. Proximity can therefore be identified as playing a role in provoking this enhanced ethic of care towards stakeholders. This further increased the likelihood of SMEs engagement with CSR. 


\subsubsection{2: Inextricable Personal and Business Connections}

The findings of this research support the personal sphere as an integral aspect of SME stakeholder networks, one of the key contributions of Spence's (2016) redrawn stakeholder theory. As one respondent explained, 'close friendships come actually across boundaries within the network'. There were numerous ways in which SMEs further interacted with the personal sphere. For example, personal connections were used to create and enhance business relationships (Spence \& Rutherfoord, 2003), with many owner-managers bringing personally associated stakeholders into the SME network.

This characteristic of SMEs resulted in relationships with multiple connections, known as social multiplexity (Lähdesmäki et al., 2017). It seems apparent then, that SME networks have 'blurred lines' and naturally integrated public and personal spheres. It was found that close and multiple connections within stakeholder networks are a relative advantage for SMEs (Lähdesmäki et al., 2017). There was also evidence to suggest an increase in moral intensity (Jones, 1991) and the social costs for unethical behaviour, stimulating the inception and engagement of CSR.

Despite supporting the inclusion of the private sphere as a territory for morality, the research also identified tensions inherent with integrated connections. For example, the difficulty to reprimand staff, as P10 said, 'when staff become your friends and colleagues it's very hard to reprimand them'. Working with family was also considered as a 'double edged sword' by P22, creating moral dilemmas and a role conflict for the owner-manager. The difficulties of this have been noted by the likes of Lähdesmäki et al. (2017) and Lähdesmäki and Suutari (2012) in relation to social capital and proximity.

The transcending of spheres also had obvious implications for CSR. Due to multiplexity in relationships, moral intensity (Jones, 1991) and stakeholder salience appeared to increase for SMEs. This intensified pressure for owner-managers to adhere to demands, resulting in moral deliberations. For example, P13 was already engaging in multiple CSR endeavours yet when asked to sponsor a school sports team, felt obliged because 'her mum was on the Marie Curie committee'. P4 
consolidates this when she said it is 'hard to say no' to CSR. For P15 the moral dilemma related to 'mates rates' for family and friends, with the majority of initial customers coming under this category, it caused tensions between her role as ownermanager and friend.

The findings show that moral deliberations did result in the involvement of nonrational emotions during decision-making in SMEs. Dyer \& Handler (1994) suggest that merged spheres create less rational behaviour than those with clear boundaries and social roles. However, emotions and personal values cannot be separated from social interactions within business (Polanyi, 1944/2001). As demonstrated by P11, the involvement of emotion when addressing business dilemmas can result in collaboration. In this case a symbiotic solution was sought in order to satisfy economic viability, stakeholder interests and the moral consciousness of the ownermanager. Wicks et al. (1994) proposed a key element of the ethic of care is emotional intelligence and "effective communication to resolve conflicts" (p. 487). The above confirms the appropriateness of this for SMEs and embraces the "nonrational' perspective of decision-making proposed by Spence (2016).

\subsubsection{3: Networks and Collaborations}

A key characteristic of SMEs is their reliance on close networks (Jenkins, 2006) and collaboration (Lähdesmäki et al., 2017). The testimony of P10 supported this when he said 'a key word for us is that collaboration, which implies this sense of reciprocity'. The common referral to collective efforts in the findings suggests that the ethic of care stakeholder perspective that puts "collaboration and cooperation as the central directive of management" (Wicks et al., 1994, p. 493) is appropriate for SMEs. In an emerging knowledge economy, the utilisation of joint ventures and knowledge sharing will be increasingly relevant for businesses, particularly SMEs.

Owner-managers identified the key advantages of collaboration as the synergistic environments and mutually beneficial outcomes. For example, for P25 and P18 collaboration mobilised projects that were bigger than their ability as a single entity. Further advantages included the provision of support, knowledge sharing and problem solving. For P23, her network was considered as 'invaluable' because it 
provided access to contacts who could 'support and signpost' to resolve issues. This reflects elements of the feminist stakeholder perspective whereby collaboration and communication is utilised to overcome conflicts (Wicks et al., 1994) and create value for the stakeholder network.

Collaboration in SMEs was sometimes a reaction to the increasing demands of stakeholders. The ability to work in partnership with other SMEs enabled P18 to 'bring a bigger offering', allowing the flexibility to react to the environment, a differentiating strength for SMEs (Spence, 2016) and in keeping with the ethic of care. This allows SMEs to "internalize the changing needs of their stakeholders" (p. 32) and create associations with other SMEs in order to adhere to these. As P18 explained, 'it's all about the joined up approach' using 'local connections' and creating alliances with other SMEs, viewing them as opportunity rather than competition.

There was a notable sense of camaraderie and perceived sense of responsibility to support fellow SMEs. The mentality was that 'small businesses shouldn't compete with each other', but support each other and compete against bigger businesses. For example, P1 was supportive of competitors because 'it just means that there's more business going on for small businesses in the areas'. This is in keeping with the caring characteristic of SMEs whereby they emphasise collaboration over conflicting competition. P1 predicts that those not open to relationships and collaborative working will 'struggle' in a future of open innovation and collaboration.

In relation to CSR, whilst engagement was largely ad hoc, there were instances whereby SMEs were engaging in collaborative efforts. Combined efforts were described by P26 whereby charitable events involved all of the local businesses, and when CSR engagement was initiated by the SME and supported by stakeholders. In P21's case, their suppliers offered services for free when for charity. Collaborative efforts were also reactive to solve issues within the local community. For example, when P18 and others applied their business skills to address the planning application for sewage works in their community. The concerted effort of these SMEs to resolve community problems shows the value of collaborative CSR. The ability of SMEs to 
address societal issues on their own may be limited, but by utilising collaboration there can be a consolidation of SME's social contribution.

\subsection{4: Summary}

To summarise, the findings of this research do align with the ethic of care perspective of stakeholder theory. An empathetic approach towards stakeholder relationships was an integral strategy of SMEs and was seen to transfer in CSR approaches whereby owner-managers felt a sense of duty towards key stakeholders. This sense of duty could be a result of relationships transcending personal and organisational boundaries for SMEs, intensifying decisions. Non-rational emotions were evident in decision-making, increasing the intensity to behave ethically and resolve conflicts, but also creating moral deliberations. SMEs utilised close networks and collaborative efforts to mobilise opportunities and provide support to increase their ability to compete with larger businesses. This combined approach was seen to transfer to CSR, increasing opportunities for engagement and strengthening the social contribution of SMEs. As the key parameters linking responsibility to SMEs and facilitating collaboration, moral proximity will be the next topic of discussion.

\section{5: Moral proximity}

The following discussion will explore the influence that different forms of proximity have on CSR, specifically referring to Jones' (1991) four typologies. Spence (2014; 2016), Lähdesmäki et al. (2017) and Lähdesmäki and Suutari (2012) will further be alluded to due to their developments on moral proximity and stakeholder salience, considering that the findings supported their assertion that proximity should be acknowledged to better explain the contextually bounded nature of CSR in SMEs. Sociological theorists such as MacIntyre (1985), Granovetter (1985) and Polanyi (1944/2001) will also be considered with reference to context, embeddedness and reciprocity. The following will cumulatively address RQ6 and RQ7, questioning the influence that moral proximity has on the inception and engagement with CSR. 


\subsection{1: Psychological Proximity}

Jaffe and Pasternak (2006) found that "intuitively, people care more about those close to them physically and emotionally" (p. 53). As owner-managers are central to SMEs and key decision-makers (Jenkins, 2006), responsibility is unavoidably connected to those actors closest to them. This elicits high levels of commitment and a feeling of closeness, otherwise known as psychological proximity (Jones, 1991; Mencl \& May, 2009). Psychological proximity was distinguishable in the findings, with examples of affective closeness coming from the likes of family, staff and the community in particular.

It is established above (see 8.4.2.2) that personal and business spheres of SMEs are inextricably linked. The multiple ties connecting stakeholders and the SME (Lähdesmäki et al., 2017) were seen to intensify moral claims and increase the salience of stakeholders (Mitchell et al., 1997). For example, affective closeness is described by P22 when she says 'I know it sounds very sentimental, but I love my father more than I loved my employees'. Family was also salient for P18 because, in his words, 'without my family's support I couldn't do it'. Finally, the consideration by $\mathrm{P} 18$ that there is a contractual relationship towards family (see 8.2.2.4) exemplifies their role as a key stakeholder and intensifies their moral claim on business operations.

One of Spence's (2016) key enhancements of stakeholder theory was the influence of proximity on stakeholder salience, adding to the urgency perspective. The urgency of claims, both temporally and criticality were referred to, particularly when considering family. For example, P13 prioritised family because as a family firm, she quotes that they were 'all breathing down my neck'. Mitchell et al. (2011) propose that, "when organisations operate at the intersections of two institutional logics, issues of urgency are potentially filtered through two often contradictory, managerial lenses" (p. 245), creating conflicts for the owner-manager. Such tensions were evident in the findings and will be discussed further in 8.5.5.

With regards to the influence of psychological proximity on CSR, the intensified salience was notable. Inception of CSR commonly came from informal networks 
closely linked to the SME, with a preference to support causes close to the ownermanager or those in their personal sphere. For example, P20 supported a chosen charity because it was something that they had a 'personal connection' with and could 'physically go there' to see the facilities they were supporting. This example partners both psychological and geographical proximity as influencing engagement. P7 further advocate causes with a personal connection, claiming 'it's not personal when you say can I have some money'. This might explain why there was a preference to donate time and skills as a more personal resource and valuable contribution from SMEs, requiring some sort of presence or personal commitment.

In summary, there were strong implications that psychological proximity is a catalyst for the SME approach to CSR. Personally relevant causes were sought, there was a preference to donate time and skills and engagement was strongly influenced by stakeholders from the private sphere. Whilst moral claims were intensified by psychological proximity and increased the likelihood of CSR requests being adhered to, this closeness did increase pressure for owner-managers due to the intersection of two contradictory, managerial lenses.

\subsection{2: Physical Proximity}

SMEs are characterised via their local embeddedness (Granovetter, 1985). It is unsurprising then that physical proximity played a determining role for CSR throughout the findings. P2 claimed that SMEs by their very nature are "more connected to a particular location' in comparison to larger businesses. He further claimed that the integrated nature of SMEs and community is the 'recipe for a more sustainable economic, social and environmental model', and a distinct advantage for SMEs. Localised and scaled CSR efforts were considered 'very very effective' by respondents, claiming that large businesses should work in partnership with their local communities to 'make CSR work'.

The CSR terminology adopted by SMEs (see 8.2.1) reinforced their physically embedded nature. Combinations inclusive of community were dominant and engagement often community focussed, showing partiality to those within geographical proximity. P2 claimed that part of the success of SMEs is due to their 
local environment, considering that the least the business can do is to give back to it. P5 supports this, describing a moral obligation to 'enhance that community and make it a better place'.

Keeping money in 'local economies' was one way that SMEs adhered to the moral obligation. As P23 said, people 'like to buy local', because they feel like 'they're supporting the economy'. This was particularly directed towards the use of local suppliers, but also evident in other ways such as the recruitment of employees from local areas. There was a strong preference to look within the 'immediate vicinity' of the SME. For P16 this inclination was even for online businesses, she said 'I'd much rather buy from a local company, even if it's online'. This suggests that despite technological advancements, there was still partiality towards those within close physical proximity.

Community focussed CSR was also reactive to overcome challenges, or as P25 describes, 'contributing to people who need it'. P18 described an example whereby localised efforts saved a social initiative through 'very hard financial times'. This was via 'direct relationships' and face-to-face interaction, as facilitated by close geographical proximity. As P2 expressed, CSR elicits a heightened 'sense of satisfaction when you do it as a community'. These findings reinforce that physical proximity and embeddedness does still play a dominant role for SMEs in the research context. It further points to the notion of reciprocity and collaboration, a fundamental premise of CSR rooted in sociological theory (Polanyi, 1944/2001).

However, Spence (2014) acknowledges that virtual organisations may not be characterised by physical proximity due to technological advancements and globalisation. This potentially dilutes the importance of geographical location. These changes are already distinguishable whereby community was conceptualised as 'wherever customers may be based', 'our customers nationally' and 'anybody and everybody upon whom my work impacts'. There are diverse and paradoxical accounts of community when the physical boundaries are no longer present. Spence (2016) questions if community will soon be represented by virtual groupings. Arguably, what is more important is discovering if the dilution of physical proximity has implications on the ethical character of the SMEs due to weakened moral 
intensity (Jones, 1991).

Finally, Mencl and May (2009) consider geographical proximity as the only form of proximity that "does not imply closeness based on "personal levels" (p. 206). However, the success of SMEs in this research was found to be reliant on the degree of legitimacy and relationships with key stakeholders of that physical vicinity (Perrini, 2006). This suggests an interrelated nature between physical and social embeddedness. Lähdesmäki et al., (2017) supports this finding and consider that physical proximity stimulates social proximity, because being spatially close favours interaction and trust building.

\subsection{3: Social Proximity}

Recent literature suggests that with regards to stakeholder salience and moral intensity, the degree of social proximity is actually more relevant than physical closeness (Courrent \& Gundolf, 2009; Lähdesmäki et al., 2017). Findings of this research do promote the value of physical proximity, suggesting that the physically embedded structure traditionally associated with SMEs acts as a 'cloud base' from which social proximity can develop. However, there were examples whereby the emphasis was not on geographical proximity, but rather a sense of common purpose.

For example, P4 was the owner-manager of a UK registered SME, yet their business operations were outsourced to Sri Lanka. Despite this geographical separation, the SME still engaged in CSR, donating old computer equipment to the community of the employees. The complex nature of proximity is exemplified when P4 described the inception of CSR, whereby employees asked if the business could donate to their 'uncle's, brother's, cousin's school'. Despite the lack of physical proximity, the social connection between owner-manager and employees, alongside the family connections of the staff, created in a pathway of proximity that resulted in the CSR act.

P21 further reflected on the role of customers, suggesting that it would not make a difference if they were located in Swansea or London as to how they would be treated. In fact, P23 would 'over-service' the clients that she had not met in order to 
nurture the relationship and 'personify the brand'. This is so that the client would 'still feel connected' despite a lack of face-to-face interaction. Lähdesmäki and Suutari (2012) contend that the essence of proximity lays in interaction. However, this research shows that does not necessarily have to be face-to-face. For P23 'it was all done over email', supporting the work of Courrent and Gundolf (2009) that suggests social proximity can be more important than physical for SMEs.

For SMEs operating online, reputation was still an important part of business, if not intensified due to the accessibility of online reviews. The above appears to suggest that physical presence is not necessarily needed to nurture this. However, due to the lack of a shop front P11 has to 'rely heavily on word of mouth' instead to ensure that 'customers are satisfied'. As P19 explains, in their niche industry 'everyone knows everyone', so personal contacts and the reputation of the individual were more important than the physical location of the business. Again, this implies that it is not just physical proximity that increases moral intensity.

To summarise, whilst physical proximity did facilitate social proximity to an extent, it was not essential. The findings show that moral intensity was not diluted due to a lack of physical presence, but positive relationships were nurtured online and over large spatial distances as driven by a common purpose. The above examples also show that different typologies of proximity are intertwined and hard to separate. Rather than one influencing CSR, it would be reasonable to assume that there are numerous forms of proximity simultaneously increasing the moral intensity of decisions and salience of stakeholders.

\subsection{4: Challenges of Moral Proximity}

There were numerous benefits of being morally proximate to stakeholders (Besser et al., 2006; Lähdesmäki et al., 2017). Examples from the findings included tangible economic benefits such as reduced costs and leniency of payments, to enhanced trust and legitimacy with stakeholders. However, the challenges of too much or lack of moral proximity (Lähdesmäki \& Suutari, 2012) were evident. As P27 announced, this makes it 'difficult to know what is the socially responsible thing to do'. 
There were a number of instances whereby too much social proximity created moral deliberations. For example, when P10 found it 'hard to reprimand' staff, often turning a blind eye to things and when P13 was unable to say no to stakeholder CSR requests due to personal relational ties. On this occasion, social proximity increased the likelihood of CSR inception, although arguably not as a result of the right moral impulses. Lähdesmäki \& Suutari (2012) contend that too much social proximity can actually facilitate unethical structures. Furthermore, close social connections were also seen to be inhibiting for the initiation of CSR because of known associations. P23 was not really contacted with CSR requests because stakeholders know her 'alliance' with a local charity. In this case, increased social proximity inhibited CSR requests from stakeholders.

Physical closeness to stakeholders also created challenges. For example, a number of respondents with premises embedded in the community described an escalation of CSR requests. For P26 the influx of requests 'plagued' her SME with stakeholders not realising her engagement with other CSR initiatives. This was challenging because of the potential negative word of mouth if requests were denied. Because of this risk and the resource constraints, SMEs appeared to hold back from supporting too many CSR efforts so as not to 'open the floodgates' and create an unmanageable number of requests. The above findings identify a proximity paradox whereby there can be detrimental aspects of close proximity for SMEs.

Alternatively, a lack of proximity also created moral dilemmas. For example, P19 felt guilty when unable to provide the same level of support to staff that were physically located further away. Furthermore, P18 considered that a lack of physical proximity could be a potential inhibitor for the inception of CSR when there is no community facing 'shop front'. He believed that if he did have a physical community presence, people would be more inclined to 'knock on the door' and ask for sponsorship requests. Moreover, despite SMEs utilising collaborative CSR efforts, there were no examples of this with geographically dispersed SMEs. This suggests collaborative CSR engagement is less likely when there is a lack of physical proximity. 
Discussions on the challenges of moral proximity identified notable moral deliberations for owner-managers, influencing the inception and engagement of CSR. This confirms that indeed, there does seem to be a form of proximity paradox. At times the increased moral intensity had a positive effect that heightened the propensity to behave ethically, yet there were also occasions whereby a dark side to proximity was identified, such as escalating pressure on owner-managers (Lähdesmäki et al., 2017; Lähdesmäki \& Suutari, 2012; Mitchell et al., 2011). Future research could therefore investigate if there are optimal levels of moral proximity to facilitate CSR.

\subsection{5: Summary}

In summary, psychological, physical and social proximity notably impacted decisions on CSR. Cultural proximity was not so evident, but arguably this could be because of the "theoretical overlap" (Mencl \& May, 2009, p. 206) with the other dimensions. Despite the changing conceptualisation of community, geographical proximity was still important for SMEs in the research context. It stimulated webs of social interaction and collaboration due to the intensity and face-to-face communication between different actors. However, with the changing structure of community and physical embeddedness this was diluting in importance.

Social proximity does appear to counteract a lack of geographical proximity when SMEs share commonalities with stakeholders. Owner-managers still felt a moral obligation to behave ethically and interact with CSR initiatives, even when there was not a physical business presence. That being said, a lack of geographical presence does interfere with the inflow of CSR requests, potentially limiting engagement. Psychological proximity also impacted CSR as participants showed partiality towards causes that appealed on an affective level. However, it notably created moral dilemmas, putting pressure on owner-managers and confirming that the dark side to proximity was very much present.

An important finding showed that these different typologies of proximity were not influencing CSR in isolation. Most commonly they were mutually reinforcing, intensifying stakeholder salience, increasing the moral intensity of decisions and 
amplifying pressure on SMEs to behave ethically. With regards to CSR engagement, this was often influenced by pathways of proximity, linking numerous different actors to the different interactions that led to engagement with the CSR act.

Subsequently, the applicability of moral proximity for CSR in SMEs is warranted. Not only does it complement the ethic of care approach that has been identified as characterising SMEs, but also as MacIntyre (1985) contends, virtues cannot be dealt with in isolation to their context. SMEs are not isolated entities due to the influence of actors within their physical and social spheres, therefore CSR should not be considered as separate from the contextual influences of moral proximity.

\section{6: The Impact of CSR by SMEs}

The final section of this discussion will summarise the perceived impact of SME's CSR engagement towards societal wellbeing. The focus will first be on the relevance of formalising CSR, referring to Murillo and Lozano (2006), Fassin (2008) and Russo and Perrini (2010). CSR communication is then considered in relation to this, with recent literature by Baumann-Pauly et al. (2013) and Wickert et al. (2016) drawn upon. The third contemplates the institutionalisation of CSR in the UK (Spence \& Perrini, 2009) and the fourth explores the perceived impact of CSR in relation to Torugsa et al. (2013) and Morsing and Perrini (2009). To conclude, a shift in mindset is proposed, reflecting on the need for education and dissemination of knowledge. The aforementioned will address RQ8 and RQ9 to consider the impact of SME's CSR efforts and how support can be provided to facilitate socially responsible practises in the future.

\subsection{1: The Formalisation of CSR}

The recognition of SMEs' growing significance has led to an emphasis on their social impact (Jenkins, 2004). Despite increasing pressure to formalise their social and environmental engagement, findings of this research show that SMEs generally do not plan, report or 'keep log' of CSR in any manner. P22 explains that 'it just happens', describing engagement as 'sporadic'. Consequently, CSR was not 
frequently budgeted for and when it was, it was more of a 'rough guide' that was not strictly adhered to. For P2, the budget was actually put in place in order to ensure expenditure on social issues rather than control it.

One of the challenges of CSR articulated by SMEs in section 5.9 was limited time and resources. This is likely to be a key explanatory factor for the lack of formalisation of social initiatives for SMEs (Russo \& Perrini, 2010). Ownermanagers perceived methods of social reporting as an administrative burden and “external imposition" (Murillo \& Lozano, 2006, p. 238). However, just because SMEs are not reporting their CSR, does not mean that they are not engaging with the concept. The findings revealed a spectrum of unique social engagement, personalised according to the SME's conceptualisation of CSR (Murillo \& Lozano, 2006). This research therefore supports the view that it is formal tools of reporting that are ineffective for SMEs (Russo \& Perrini, 2010).

A further challenge was the alternative language used for CSR, resulting in a “confused notion" (Fassin, 2008, p. 367) of CSR as outlined in section 8.2.1. This potentially disguises the true impact of SME engagement. Despite the limited exposure of CSR, the findings showed a perceived ethical superiority of SMEs over large businesses. CSR was considered a large firm concept, with the perception of greenwashing and "mock compliance" (Holliday, 1995, p. 228) to fulfil the interests of the business rather than society. As P18 explained, a lot of people 'do the right thing, but for the wrong motivations', undermining the engagement. This proves Fassin's (2008) claims that reports to not prove "superior ethical behaviour" (p. $364)$.

The quality movement demanding compliance and standardisation is not in line with the general nature of SMEs. For SMEs in this research, CSR was not considered as something that should be restricted to departments (Fassin, 2008), but an embedded concept. Consequently, instead of standardisation, CSR for SMEs was aligned with their context and individual interests. Present analysis has suggested that reporting influences behaviour, yet the above supports Fassin (2008) when he proposed this as in fact a "fallacy" (p. 365) for SMEs. 


\subsection{2: Communication of CSR}

Linked to the formalisation of CSR is the external communication of initiatives. The findings of this research support the recent work of Wickert et al. (2016) who suggest that CSR 'walk' and 'talk' are incongruent for SMEs, whereby CSR is implemented silently (Nielsen \& Thomsen, 2009). For example, there were occasions when SMEs consciously decided to maintain low visibility such as P13, who did not want publicity for their CSR engagement and P14, who wanted to stay 'as anonymous as possible'. Yet for many, it was simply not realising the valuable contribution that they were making. As P9 said, SMEs 'do lots of good stuff' but 'don't even realise'. This means that they are 'not recognised for what they do' and do not promote it.

Another reason identified for the lack of CSR communication is external stakeholder expectations. P27 suggests that you can voluntarily communicate CSR engagement on websites whereby 'you're not paying someone to accredit you'. However, despite this form of communication being free and voluntary, SMEs were wary of the increased expectations and scrutiny from stakeholders that comes with it. If the claims were then not adhered to, an implementation gap would be present (Wickert et al., 2016) and stakeholders would hold the SME accountable.

That being said, proximity to stakeholders and a reliance on social capital arguably form more genuine communication channels for SMEs. Therefore, SMEs do not need to rely on formalisation and external reporting. Instead, CSR is translated and validated through close stakeholders, who can visibly see the social engagement, rather than just receiving communication about it. This supports Russo and Perrini (2010) when they say, "SMEs remain unable to formalise such relational capital through managerial tools" (p. 217), yet opposes their view that this inhibits their value creation.

As Fassin (2008) says, for large businesses "much communication around CSR is subjective" (p. 367) and reliant on self-written reports or manager's perceptions. Therefore, they only partially represent the company's CSR operation (Perrini, 2006). It could be concluded then that the communication and stakeholder 
validation in SMEs is a more valuable and reliable form of CSR communication. To conclude, the lack of communication and policy documents do not invalidate the social endeavours and collective contribution that SMEs have to society.

\subsection{3: Institutionalisation of CSR by SMEs}

Elements of CSR are increasingly being institutionalised within organisations, social systems and society. In the UK, the government's role to create an environment conducive to CSR is developed in comparison to many other countries (Spence \& Perrini, 2009). For example, in Wales specifically, the introduction of The Wellbeing of Future Generations Act (2015) is a massive step in terms of legislating CSR for public bodies. Despite this, the findings still revealed an inconsistency of CSR inclusion within formal processes. For example, P25 had never been asked to codify CSR in any government tender, despite it potentially making a big difference to their business operations.

On the other hand, P21 had recently come across a vender proposal asking for evidence of their 'formal process' of CSR. The owner-manager viewed the request as positive and was interested to see if it became a 'growing trend'. Participants proposed that the new legislation in Wales is likely to enforce consistency of CSR codification beyond public sector institutions and impact SMEs. Whilst the formal request for CSR processes could be beneficial to expose the true social value of SMEs, the attempts to standardise and restrict them to prescribed CSR categories does not reflect the nature of their CSR engagement. Encouragement of consistency and conformity does not allow for the contextual, resource based approach to CSR that SMEs have adopted.

That being said, one of the main issues with CSR and the reason for conceptual confusion is the lack of a common framework. As P25 explains, there is a 'wider issue of measurement', with no 'common framework' that SMEs understand. He continued to suggest that once there is a common language 'you can measure against that', but with CSR 'it's so wide'. A paradox has been identified here between the benefits of institutionalisation and development of a formal CSR framework and the 
desire to nurture the contextual nature of SMEs and their perceived ethically superior conceptualisation of CSR.

To bring the concept back to its moral foundations, this research suggests that the institutionalisation of CSR needs to be rooted at the micro level so that morality is embedded within corporate structures. As P23 proposes, SMEs need to embed values at the core of the business during start up, rather than retrospectively trying to 'fix it'. This will codify and habituate social responsibility as an accepted norm in the organisational system and encourage consistency. It will also allow for contextual differences, not limiting SMEs to prescribed and homogenous engagement.

To summarise, there are government structures in place that are important to support SMEs and consequently their CSR engagement. However, respondents claim that policy makers still 'need educating' about the characteristics and needs of smaller businesses. The institutionalisation of CSR in the UK and Wales is progressively impacting SMEs and with the introduction of legislation this is likely to maintain momentum. However, traditional routes of formalisation risk diminishing the moral nature of CSR in SMEs, turning it into a compliance exercise. Consequently, there is a need to introduce an institutional lens of CSR that frames the concept adequately for SMEs to implement at firm level.

\subsection{4: Perceived Impact and Benefit of CSR by SMEs}

Baden and Harwood (2013) argue that time is better spent looking at the social impact of CSR rather than financial. With regards to this, there was a consciousness in the findings that CSR needs to be 'better directed' to ensure that it actually impacts recipients. P17 suggests that it is a personal responsibility when engaging in CSR to ask questions such as 'who is it actually helping?' He continues to proclaim that SMEs are collectively contributing massively to society and engaging in valuable CSR initiatives, 'but that in itself is not sustainable in the longer term'. The long-term benefit of these initiatives could therefore be questioned. 
SMEs did engage in a number of CSR initiatives directed at solving community problems longer term, but engagement was still largely ad hoc and philanthropic. The research proposes that this should be challenged and SMEs made aware of the scope of CSR and need for proactive approaches that "actively support sustainable economic, social and environmental development” (Torugsa et al., 2013, p. 383). Although SMEs were more likely to adopt scalable community based approaches, it would be beneficial for them to "thoughtfully and intentionally consider what social problem(s) they can solve" to "leverage the power of their enterprise" (Roche \& Jakub, 2017, p. 159).

However, P15 points out that boundaries are blurred when establishing at what point a social decision becomes a commercial decision in SMEs. By accepting the two as inextricably connected and instead focusing on transforming perspectives to consider sustainable impact, it could be more valuable for their communities. Whilst CSR for wealth creation is not advocated by SMEs, Windsor (2001) makes a notable point whereby "managers should understand that there are serious deviations between short run impacts of business activities and the long run alignment of business and social interests in wealth creation" (p. 250).

\subsection{5: Educating and the Dissemination of Knowledge}

Spence and Perrini (2009) claim, "it is the role of policy makers and organisations to spread the idea of socially responsible behaviour and CSR practises" (p. 28). Yet as P25 announced, SMEs are by and large 'pretty rubbish' at selling their own virtues. Discussions above identify that SMEs silently implement CSR and therefore do not disseminate their conception of it. Instead, there was a reliance on organisations such as the FSB and Business in the Community (BITC). The challenge is therefore to create mechanisms to disseminate the contribution of SMEs and promote their conception of socially responsible behaviour.

A difficulty identified by P8 is reaching out and educating SMEs that are not aware of CSR. He proposes a need to disseminate understanding to 'the wider audience', concluding that 'there are a lot of people willing out there, but they just don't know it'. A suggested mechanism to raise awareness is through events and manifestos of 
the support organisations mentioned above. However, it is also the responsibility of owner-managers to disseminate knowledge, particularly those engaged in best practice (Jenkins, 2004). P18 identified 'existing business owners' as the 'best resource' accessible to SMEs, with P25 suggesting that 'you learn more and trust more from your peers'. Owner-managers are consequently best placed to promote a common understanding of CSR to those that they can immediate influence (Murillo \& Lozano, 2006).

However, P24 proposed that a bigger challenge is educating and changing the mindset of society. She believed that there is an issue whereby 'we only value something that we can put monetary value on'. This economically rational view originating from Weber (1930/2003) is considered a 'big failing' and requires a 'change of mindset' so that social contributions are also valued. P18 believes that it is the responsibility of individuals to 'influence and change people's attitudes' as a collective.

This aligns with Roche and Jakub (2017) who observe an increased awareness of the new social role of business in society, showing a "reassuring sign of the capacity of humanity to adapt and adjust to new realities" (pp. 160-161). P24 has noted observations whereby 'young people seem to be less profit driven and more driven by other things'. This highlighted the education of new generations as an important way to nurture a socially responsible mindset from the outset rather than having to adapt to one.

\subsection{6: Summary}

In summary, there is increasing pressure for SMEs to formalise CSR, yet current reporting procedures are ineffective and not in line with SME characteristics. Despite this, the silent implementation and lack of formalisation of CSR does not invalidate the efforts of SMEs. Instead, business activities are translated and validated through close stakeholder networks. Stakeholders can visibly see the impact, rather than just hearing about it, positioning SMEs CSR efforts as genuine compared to the incongruent implementation of large businesses. That being said, 
the lack of a common framework for CSR does create confusion for SMEs and puts the true value of SMEs social engagement at risk of not being realised.

Despite the valuable social contribution from SMEs, it was identified that there is still a need to educate on the scope of CSR. This will help assist SMEs to move away from the ad hoc and philanthropic nature of engagement, towards conscious consideration of the long run impact for recipients and society. It is argued that the institutionalisation of CSR at a firm level is a valuable way to infiltrate sustainable CSR into reality. There is a paradox whereby attempting to institutionalise CSR for SMEs defies their contextual nature, restricting them to prescribed norms of engagement. By focusing on the firm level, this accommodates the unique context of SMEs in line with the ethic of care and moral proximity developments.

SME support organisations can play an important role to disseminate the value of socially responsible behaviour. However, arguably it is more important that ownermanagers spread the SME conception of CSR to their peers. It has been identified that SMEs are driven by moral and relational elements rather than economic, with CSR considered as a concept that should be embedded within the core of institutions. The communication of this morally grounded conception will be valuable to trigger a systematic reformation of thinking for businesses of all sizes, in order to re-moralise CSR as an ethical concept.

To conclude, attention does seem to be turning away from the financial to social impact of CSR. However, there is still more that can be done to embed a morally grounded conception of CSR. As Fassin (2008) said, "the essense of CSR lies in the implementation of responsible business practices at all levels of the corporation; it lies in the corporate culture, not formalization" (p. 385). It is therefore important to create an environment that nurtures CSR in SMEs by creating tools differentiated from those of large businesses and mechanisms that disseminate their valuable contribution.

\section{7: Chapter Conclusion}


To conclude, this chapter has presented a discussion of the findings in relation to the five key research objectives and literature reviewed in Chapters Two and Three. Owner-mangers conception of CSR was overtly moral or community centred, broadly supporting the redrawn domains of responsibility proposed by Spence (2016) and confirming the relevance of the ethic of care to explain CSR in SMEs. However, the redrawn model itself does not seem to admit the full complexity of the concept. A number of considerations are therefore proposed, the most important being that the freedom to subsume morality to economic pursuit implies that responsible behaviour is only pursued if the SME has margin to do so.

The CSR categorisations proposed by Wickert et al. (2016) were described as simple, yet the drivers of CSR were distinctly complex and influenced by intrinsic and contextual factors. There was limited evidence of instrumental drivers for CSR in terms of Friedman's (1970) paradigm. Therefore, we cannot assume that SMEs are driven purely by economic rationality or profit maximisation. Broadly speaking, it was accepted that SMEs were driven by ethical or relational/organisational motivations, aligning with the ethic of care. Although, it was accepted that these classifications are in interplay and can change over time.

This research confirmed that the ethic of care and moral proximity are appropriate to explain how the key stakeholders of SMEs shape CSR. For SMEs, the salience of stakeholders and nature of relationships was multifaceted and contextual, unavoidably influenced by moral proximity. Multiple ties across personal and business spheres were beneficial, but seen to increase the intensity of relationships, resulting in non-rational emotions occurring in decision-making. These emotions increased the propensity of responsible behaviour and likelihood of CSR, but also created moral deliberations for owner-managers, revealing a dark side to proximity. Spence's (2016) redrawn stakeholder theory was useful to advance SME specific theory in relation to these characteristics. However, it could be considered an oversimplification of their reality. Suggestions are therefore made to progress the theory and accommodate the interrelated stakeholder dynamics of SMEs.

Finally, the collective contribution of SMEs is invaluable to societal wellbeing. Their silent implementation and lack of reporting does not refute their social 
contribution, but it does mean that their true value is not being realised. Nevertheless, there is still a need to educate SMEs on the scope of CSR to move away from ad hoc engagement, to proactive forms of CSR that consider the long run impact for recipients. To conclude, it is important to work towards a common framework that nurtures CSR in SMEs. As Murillo and Lozano (2006) recognise, the future challenge is to find differentiated tools that accommodate and promote the social value of SMEs. 


\section{Chapter Nine: Conclusion}

\section{1: Introduction}

The purpose of this final chapter is to present the conclusions of the thesis. This research set out to explore how CSR is constructed and practised in SMEs, specifically looking at the role of moral perspectives to better explain the concept. In order to address this, the chapter begins with a review of the broader research aims and objectives that were established in Chapter One. Following this, the thesis is summarised by discussing fundamental themes and highlighting the key theoretical, practical and methodological contributions of the research. The chapter then concludes by considering the limitations of the study and identifying directions for future enquiry, followed by some closing remarks.

\section{2: Revisiting the Research Aim, Objectives and Questions}

The research aim, objectives and questions were refined during the development of this thesis. The main emphasis was on the concept and practise of CSR in SMEs and the reclamation of its moral foundations. Accordingly, the main research aim was as follows:

To develop an understanding of how CSR is constructed and practised in SMEs and the role of moral perspectives to explain such engagement.

In order to address this overall aim, five research objectives were formulated with nine corresponding research questions. These responded to ongoing issues and unanswered questions previously identified in the literature. Therefore, the following section presents the key findings and contributions to knowledge in line with these areas of focus and the key research objectives.

\section{3: Findings and Overview of the Research}


This section of the conclusion provides a summary of fundamental themes and conceptual contributions of the research. These were to account for gaps and unresolved areas of enquiry in the literature including; the (1) lack of SME specific research for CSR, (2) the under acknowledged contribution of SMEs to the economy and society, (3) a distinct lack of theoretical developments for CSR in SMEs specifically, (4) a lack of context sensitive research approaches relevant for SMEs, (5) a need to reclaim the moral foundations of CSR and (6) a lack of empirical exploration of Spence's (2016) redrawn theories. Identification of the gaps in the literature has emphasised the room to progress this research agenda and provide avenues for future exploration.

\section{RO1: To evaluate how SME owner-managers construct and make sense of their role towards society}

RO1 was framed by two research questions, (RQ1) to discover how SME ownermanagers construct and make sense of their role towards society and (RQ2) to establish the relevance of the ethic of care to explain their conception. In answering the research questions, it was identifiable that owner-managers were aware of their role in society, with every respondent engaging in some form of socially responsible activity. This was a reflection of their moral consciousness as individuals and close stakeholder relationships that contributed to shaping their perceptions. Consequently, the conceptualisation of CSR was bound by both the social realities of the ownermanager and personalised to the context of the SME. This meant that there were multiple influencing factors that resulted in diverse terminology and definitions of CSR, as identified in section 8.2.1.

The terminology used by owner-managers indicated that CSR is constructed as an overtly moral or relational concept, still centred on community. With regards to RQ2, corresponding features from the ethic of care (Spence, 2016) were confirmed within their conceptualisation. For example, (1) SMEs were profit satisficers, pursuing survival rather than profit maximisation, (2) care towards stakeholders went beyond the societal standards associated with ethical responsibilities, (3) CSR was still largely philanthropic, driven by personal experience and stakeholder influence and (4) there was an emphasis on personal integrity and an individual responsibility 
to behave ethically. This identified the need for morality to be considered as the basis of reasoning for all conversations regarding CSR.

However, there were two different perceptions of CSR from owner-managers. The first was CSR as an embedded concept and the second that CSR is something engaged with, providing it does no harm to the business. The second identifies a perceived trade off, suggesting that CSR is only adhered to if SMEs have the margin to do so. This hints at how economic rationality has subordinated morality over time (Fevre, 2003), but also that morality is still present. The research identified a need to diverge from the economically rational conception that follows assertions from Friedman (1970). In line with this, the findings highlight that currently the SBSR pyramids (Spence, 2016) give SMEs the freedom to prioritise economic satisfaction over personal integrity. Therefore, it is identified that enhancements can be made to the theory to prioritise the moral foundations of CSR, rather than economic.

\section{RO2: To investigate the key motivations and drivers for CSR in SMEs}

R02 was guided by (RQ3) questioning the key motivations and drivers of CSR. This was to establish if CSR in SMEs is motivated as an economically rational construct, or moral. The findings of the research identify that SME perspectives were primarily ethical or relational/organisational, with limited evidence of instrumental perspectives. Consequently, we cannot assume that SMEs are driven by economic rationality or profit maximisation. However, drivers were distinctly complex and influenced the inception and engagement of CSR through both intrinsic and contextual factors. For example, respondents often referred to intrinsic motivations such as personal interests, a moral obligation towards stakeholders or feeling personally accountable for diminishing resources.

However, SMEs were also evidently defined by their context and relational/organisation factors within. For example, they reactively addressed CSR requests from stakeholders, proactively pursued CSR for relational benefits, or simply recognised reciprocal benefits of CSR engagement, even if these were not actively sought. To add to the complication of categorising drivers, it was found that motivations are dynamic and interactive. This might explain the sense of perplexity 
and paradoxical accounts of drivers from owner-managers. Societal expectations and organisational circumstances are constantly re-adapting, therefore CSR drivers can be considered as working simultaneously or on a continuum, with some more salient than others at different moments in time.

\section{RO3: To explore CSR engagement within SMEs and the role of key stakeholders in shaping such practices}

It was also identified in the literature that there was a lack of contextually sensitive theories for CSR in SMEs. Consequently, R03 set out to establish the influence of context by asking (RQ4) how stakeholders are prioritised, (RQ5) the nature of relationships with stakeholders and the influence of these on the inception and engagement of CSR. The findings show that stakeholder theory is multifaceted and complex for SMEs. Stakeholder prioritisation went far beyond the simple classifications articulated in previous research. SMEs made sense and prioritised based on their constructions of reality, whereby individuals are submerged in social relationships with stakeholders that transcend both personal and business spheres (Polanyi, 1944/2001). It was recognised that stakeholder salience is a reflection of current circumstances and therefore prioritised depending on who has the most urgent claim.

Spence's (2016) redrawn stakeholder theory addresses the need for contextually sensitive theory for SMEs. However, the research revealed a number of ways that this could be enhanced. For example, whilst the purposeful simplifications from the author are acknowledged, it cannot be denied that the simplistic categorisations and one-dimensional stakeholder map misrepresents the varied nature of stakeholders. It is proposed that interrelated webs of stakeholders enable the business to operate and concurrently influence CSR, explaining why owner-managers often showed a difficulty to simultaneously manage and prioritise stakeholders.

Finally, the nature of SME relationships confirms the appropriateness of the ethic of care. Stakeholder relationships were found to transcend personal and business spheres, resulting in multiple social ties between the SME and stakeholders. This elicited non-rational emotions in decision-making, increasing the intensity of 
decisions and need to behave ethically, but also creating moral deliberations for the owner-manager. However, SMEs also utilised these close relationships through networks and collaborative efforts, to mobilise opportunities bigger than their ability as a single entity. The importance of collaboration and mutually beneficial relationships was therefore emphasised for SMEs in the present and future.

\section{RO4: To explore the role that moral proximity to key stakeholders can play in influencing or inhibiting CSR practices in SMEs}

RO4 addresses the need for contextually sensitive theoretical approaches to explain CSR in SMEs. It does this by questioning the influence of moral proximity on the (RQ6) conceptualisation and (RQ7) inception and engagement of CSR. It also explores moral proximity qualitatively, an alternative to the quantitative enquiry dominating research in the past. Broadly speaking, moral proximity was confirmed as appropriate to explain CSR in SMEs. Moral issues cannot be dealt with in isolation to their context and therefore CSR should not be considered as separate from the parameters that link responsibility with the SME. This thought stems from the sociological perspective of MacIntyre (1985).

Geographical proximity was still found to be important and community embeddedness a pervasive characteristic of SMEs. Most notably, the physical closeness intensified the salience of moral issues and stimulated collaboration through webs of social interactions, enabled by face-to-face interaction. However, a key finding was the changing conception of community. Geographical embeddedness appeared to be diluting in importance as technology advances and businesses are turning to online operations.

The implications of this reduced physical proximity are unknown in relation to CSR in SMEs. Nevertheless, this research contributes by suggesting that social proximity does counteract a lack of physical proximity when there are shared commonalities. Owner-managers did still feel a sense of moral obligation to behave ethically and engage in CSR, even when geographically disperse, sometimes even increasing the need to nurture relationships. However, a lack of physical presence did interfere with the inception of CSR, potentially reducing engagement. 
A further contribution brings attention to the proximity paradox identified. Whilst there were many benefits of moral proximity, closeness to stakeholders also created tension and role conflicts for owner-managers. It was found that typologies of proximity do not act in isolation and are mutually reinforcing, intensifying decisions for SMEs. For example, whilst many SMEs were reliant on psychological closeness to certain stakeholders, it also increased pressure for them to engage with CSR requests. It would therefore be interesting to investigate if there are optimal levels of moral proximity to facilitate CSR, without creating undue tension for ownermanagers.

\section{RO5: To summarise the perceived and potential impact of SME CSR initiatives}

RO5 addresses the overlooked contribution of SMEs to the economy and society and the need to recognise their social impact as well as financial. This questions (RQ8) the influence of CSR in SMEs on societal wellbeing and asks (RQ9) how it can be supported in the future. The findings indicate that despite the lack of formalisation, the social contribution of SMEs is significant. However, CSR could be better directed to ensure it is having a positive impact for recipients. The research proposes that the sporadic, philanthropic engagement still observed should be challenged to leverage sustainable CSR initiatives. It is arguable that society as an ecosystem is only as strong as its weakest link; therefore it would be beneficial if CSR, when possible, thoughtfully and intentionally met the needs of communities that need assistance.

However, the lack of a consistent framework and silent implementation of CSR has disguised the true impact of SMEs. Respondents suggest that policy makers need educating on the needs of SMEs, as institutionalisation of CSR is inconsistent in government structures and standardisation not in line with their characteristics. Instead, SMEs rely on validation from close stakeholders who can see the impact of their engagement. In order to work towards a common framework for SMEs, there is a need for differentiated tools and communication mechanisms. A key consideration is that CSR should be institutionalised at a firm level, so that morality is embedded within corporate structures during start up and normalised as a reality, rather than retrospectively trying to fix it. 
Finally, it was considered an individual responsibility to influence people's attitudes. Owner-managers are still considered the best resource to disseminate knowledge. By encouraging the dissemination of the ethical conception of CSR evident in SMEs, it could make visible the true value of their social impact, recreating CSR as a moral concept. Attention does seem to be turning to the social impact of CSR, but there is more that can be done to change the mindset of society to value these social contributions. The research highlighted that by focusing on communication from support organisations such as the FSB and BITC, and the education of new generations, it could nurture a socially responsible mind-set from the outset rather than having to adapt to one.

\subsection{Contributions to Knowledge}

\section{Theoretical}

The following section discusses how the findings have contributed to theoretical, practical and methodological knowledge. On a theoretical level, the main motivation of this thesis was to address an ongoing issue in the literature that suggests a need for SME specific CSR research. First, as discussed in Chapter One and Chapter Three, past CSR research has somewhat overlooked the distinct perspectives of SMEs. Whilst there has been a shift in attention and rise of descriptive research, this thesis develops the discourse by empirically exploring and confirming the appropriateness of two theoretical perspectives: (1) the ethic of care and (2) moral proximity (Spence, 2014). These were identified as theories that accommodate for context and acknowledge unique characteristics, behaviours and relationships inherent in SMEs.

Second, the need for contextually sensitive theorisation of CSR in SMEs led the researcher to explore the empirical appropriateness of these perspectives via two redrawn CSR theories: (1) Spence's (2016) SBSR pyramids and (2) stakeholder theory. The research extends knowledge first by confirming the theoretical value of the moral perspectives to inform understanding of CSR, and second by making suggestions to enhance the conceptual work to better reflect the complexity of the 
concept. It further contributes as the first study to empirically explore the theory crafted by Spence (2016) and by offering a morally grounded theoretical base that future CSR research in SMEs can build from.

Third, there have been recent calls to reclaim the moral foundations of CSR. As established in Chapter Two and Three, over time there has been a subordination of morality to economic rationality. This can be seen in the CSR literature whereby it has been constricted by economically rational justifications and an obsession over proving the business case. Literature suggests that the emphasis on instrumental outcomes has driven out the intrinsic motivations of CSR, therefore it is argued that a refocus on the social sciences is more important than advancing the business case in CSR research (Baden \& Harwood, 2013; Brooks, 2010; Spence, 2016). This research contributes by opening the door to moral theories for CSR and confirming their relevance.

To address the above issue and contribute theoretically, Chapter Two is dedicated to the sociology of economic behaviour. This located CSR in the wider context and considered the sociological understanding of business and society relations to date. In doing so, it allowed a deeper comprehension of economic rationality and morality as key topics underpinning CSR. Contributions from this discourse show commonalities with other CSR related disciplines, implying the need to reclaim the moral foundations of society. However, to critique economic rationality, it requires the understanding of its philosophical origin from sociology, as developed in Chapter Two.

Furthermore, the whole premise of Polanyi's (1944/2001) work was based on the inability to deal with moral acts in isolation to their context. This sociological viewpoint supports the theoretical value of the ethic of care and moral proximity because of their focus on context and relationships, rather than principles and outcomes. The multidimensional framework of analysis also remains sensitive to intrinsic, organisational and wider political, social and cultural implications of the research. Both of these contribute by addressing the issue of decontextualisation in SME research. 


\section{Practical and Policy}

On a practical and policy level, the research findings provide guidance for policy makers and practitioners in the UK and inform academic debate. This guidance will be important to accommodate the unique conception of SMEs as pressure increases for them to formalise their CSR engagement. The research contributes to practice by confirming key characteristics of SMEs, their understanding and perspectives of CSR and outlining current challenges that they face when engaging with the concept. By bringing awareness to CSR in the UK context, it has potential to reach SMEs not currently engaging with their social responsibilities. There is also a possibility that the attention will help to redirect those engaging with sporadic, philanthropic CSR, to consciously consider solving social issues within their context. Collectively, this will help to leverage social, environmental and economic development and infiltrate sustainable CSR into reality.

On a policy level, this research indicates that the attention on the social activities of SMEs is only going to increase. However, the definitional ambiguity, lack of common framework and ineffective formalisation mechanisms are persistently deterring SMEs from getting the recognition that they deserve. The research contributes by suggesting that if the UK wants to work towards a common institutional framework for CSR, the power of language needs to be acknowledged. For example, the term CSR should be adopted consistently or progressively adapted to one closer to the SME conception.

A further contribution is the focus on educating UK policy makers and practitioners on the specific characteristics and needs of SMEs. This will aid the formulation of relevant mechanisms to facilitate a unified conceptual understanding and communication channels that promote the value of their social impact. Support organisations such as the FSB and BITC were found to be important to assist this process and disseminate information. However, on a larger scale one of the biggest challenges is the need to transform the mindset of society to one that values social contributions as well as economic. This research contributes by emphasising the need for education in schools that nurtures a socially responsible mindset from the outset, rather than having to adapt to one. 


\section{Methodological}

On a methodological level, the research makes a contribution by adopting a social constructionist approach, considering CSR as a socially centred phenomenon. This enabled deep exploration of the conceptualisation and engagement of CSR in the UK setting, drawing from the interpretive repertoires of SME owner-managers. The methodological perspective played a necessary role to convey multiple interpretations of CSR and uncover detail that may have been missed by quantitative methodologies. This was a particularly significant aspect of the research considering that there is not a consistent definition or framework for CSR.

Furthermore, CSR literature has previously been dominated by quantitative enquiry, with SME specific research often dictated by scaled, large firm perspectives. These do not respond to the differences within and between SMEs, or accommodate the perspectives of the actors engaging with the concept (Holliday, 1995; Moore \& Spence, 2006; Spence, 2007; Spence \& Painter-Morland, 2010). All of these aspects indicate that the qualitative approach was a suitable and necessary research strategy to capture the complex dynamics of CSR and multiple realities influencing it.

Finally, the framework developed by Deetz $(1994,1996)$ allowed methodological reflection and fluidity in the research. The use of this framework enabled an articulation of the movement from broadly emergent, to a priori and oscillations between both during the analysis of the data. This gave the methodology sufficient flexibility to tell the story of the research, rather than direct it. By doing this, the meaning of CSR could be articulated in the language of owner-managers, leaving scope for a moral dimension. This emergent aspect of the research was an important contribution to build propositions and disprove or confirm the two theoretical perspectives being explored.

\section{5: Limitations of the Study and Directions for Future Research}

Whilst this study provides an enriched understanding of CSR in SMEs, as with all research, there are a number of limitations that provide scope for future studies. 
First, the research evaluates CSR in SMEs from the perspective of the ownermanager. The social construction of CSR is therefore placed on the intrinsic processes of one individual, despite the desire to evaluate the CSR perspective of the whole organisation. Whilst owner-managers are most commonly principal and agent in SMEs, it could be flawed to assume that on all occasions, their perspective of CSR permeates the organisation without mediation from others. As such, this may not be a fair representation of the SME as a whole. Consequently, a more focused attempt to seek multiple stakeholder perspectives would be a suitable avenue of exploration.

A second limitation is that the research does not seek the perspectives of recipients of CSR. There are already issues inherent with formalising engagement, without the additional pressure of impact assessments. However, the research uncovered a consciousness that CSR could be better directed to impact recipients in a more sustainable manner. The opinions of those benefiting from CSR are not yet represented in the research agenda, yet they could contribute to research and practice by providing alternative perspectives on the perceived social impact from CSR in SMEs.

A third consideration is that whilst this research is conducted across various sectors, it is confined to the UK setting. This context was discussed in Chapter One and established as fertile ground for CSR research. That being said, cross-country comparisons would have been warranted to identify differences between the UK and other contexts. Furthermore, due to the exploratory nature of the research and limited time frame, cross-sector analysis was not included. Industrial sector has been identified as a key distinguishing feature between SMEs, therefore CSR engagement is likely to be influenced by the norms of the particular sector. This could be a pertinent consideration for future research.

Fourth, a relative position has been taken in this research, seeking to describe SMEs loosely rather than pointing to a definitive or more specific subset. SMEs are heterogeneous and there are likely to be differences between the CSR perspectives of micro to medium-sized businesses, different industries and organisations operating online for example. Consequently, in order to acknowledge the idiosyncrasies between SMEs, a more focused or comparative study distinguishing between these 
variables would be merited. In terms of this research, a focus on SMEs operating online could prove interesting to establish the implications of reduced physical proximity on CSR engagement.

Finally, this research empirically confirmed and proposed enhancements of the two moral perspectives and redrawn theories proposed by Spence $(2014,2016)$. Whilst this process was valuable, attempts were not made to extend or develop drastically new theories. Further research confirming these moral perspectives would therefore be beneficial to validate the findings of the study and build on the insights of this research. Nevertheless, the research did open up pathways for future work to build on and continue to expand theory for CSR in SMEs.

\section{6: Concluding Remarks}

This chapter has presented the conclusions of this thesis, revisiting the research aim and objectives, providing an overview of the research and drawing key conclusions from the findings. The main theoretical, practical and methodological contributions have been discussed and propositions for future research outlined.

In relation to the main research aim, the results demonstrate that the ethic of care and moral proximity are appropriate moral perspectives to explain CSR engagement in SMEs. The construction and practice of CSR was influenced by a number of factors on different levels of analysis. The first was the integral role of the owner-manager. Their intrinsic beliefs, values and interests inevitably influenced the inception and engagement of CSR within the organisation. At the organisational level, the context of the SME, moral proximity to stakeholders and the interrelated nature of relationships largely shaped CSR and increased the intensity to behave ethically. Finally, the institutional level recognised that CSR is embedded in sociological, cultural and political structures specific to the UK research setting, all that have historically shaped the concept.

The conclusion of this research recognises that the conceptualisation of CSR is complex and dynamic, changing with the social realities of individuals. However, it 
was most commonly constructed and practised as an ethical or relational concept in SMEs, rather than an economically rational one. This suggests that there is an element of morality still existent in society. As mentioned in the discussion, there are reassuring signs that humanity has the capacity to adapt to new realities (Roche \& Jakub, 2017). This promotes the need to nurture the mindset of society and remoralise the CSR debate. CSR is now much more than philanthropy and society is at a critical moment in time whereby the concept can be leveraged towards a profound, positive, societal change. 


\section{References}

Abbott, A. (2004). Methods of Discovery: Heuristics for the Social Sciences. New York, NY: W.W Norton.

Abrahamson, E., \& Hambrick, D. C. (1997). Attentional homogeneity in industries: The effect of discretion. Journal of Organizational Behavior, 18(S1), 513-532.

Ackerman, R. W. (1973). How companies respond to social demands. Harvard Business Review, 51(4), 88-98.

Ackerman, R. W., \& Bauer, R. A. (1976). Corporate Social Responsiveness. Reston, VA: Reston Publishing.

Aguilera, R. V., Rupp, D. E., Williams, C. A., \& Ganapathi, J. (2007). Putting the S back in corporate social responsibility: A multilevel theory of social change in organizations. Academy of Management Review, 32(3), 836-863.

Ahmad, N. H., \& Ramayah, T. (2012). Does the Notion of 'Doing Well by Doing Good' Prevail Among Entrepreneurial Ventures in a Developing Nation? Journal of Business Ethics, 106(4), 479-490.

Alvesson, M., \& Deetz, S. (2000) Doing Critical Management Research. London, Sage.

Amato, L. H., \& Amato, C. H. (2007). The Effects of Firm Size and Industry on Corporate Giving. Journal of Business Ethics, 72(3), 229-241.

Anderson, A. R., \& Smith, R. (2007). The moral space in entrepreneurship: an exploration of ethical imperatives and the moral legitimacy of being enterprising. Entrepreneurship and Regional Development, 19(6), 479-497.

Anokhin, S., \& Schulze, W. S. (2009). Entrepreneurship, innovation and corruption. 
Journal of Business Venturing, 24(5), 465-476.

Argandoña, A., \& von Weltzien Hoivik, H. (2009). Corporate Social Responsibility: One Size Does Not Fit All. Collecting Evidence from Europe. Journal of Business Ethics, 89(3), 221-234.

Arvidsson, S. (2010). Communication of Corporate Social Responsibility: A Study of the Views of Management Teams in Large Companies. Journal of Business Ethics, 96(3), 339-354.

Avram, D. O., \& Kühne, S. (2008). Implementing Responsible Business Behavior from a Strategic Management Perspective: Developing a Framework for Austrian SMEs. Journal of Business Ethics, 82(2), 463-475.

Aupperle, K. E. (1984). An empirical measure of corporate social orientation. In L. E. Preston (Ed.), Research in Corporate Social Performance and Policy (467-479). Greenwich: JAI Press.

Aupperle, K. E., Carroll, A. B., \& Hatfield, J. D. (1985). An Empirical Examination of the Relationship between Corporate Social Responsibility and Profitability. Academy of Management Journal, 28(2), 446-463.

Baden, D. (2016). A reconstruction of Carroll's pyramid of corporate social responsibility for the $21^{\text {st }}$ century. International Journal of Corporate Social Responsibility, 1(8), 1-15.

Baden, D., \& Harwood, I. A. (2013). Terminology Matters: A Critical Exploration of Corporate Social Responsibility Terms. Journal of Business Ethics, 116(3), 615-627.

Baden, D., Harwood, I. A., \& Woodward, D. G. (2011). The effects of procurement policies on 'downstream' corporate social responsibility activity: Content-analytical insights into the views and actions of SME owner-managers. International Small Business Journal, 29(3), 259-277. 
Barnard, C. I. (1938). The Functions of the Executive. Cambridge, MA: Harvard university press.

Bauman, D. (2011). Evaluating Ethical Approaches to Crisis Leadership: Insights from Unintentional Harm Research. Journal of Business Ethics, 98(2), 281-295.

Bauman, Z. (1993). Postmodern Ethics. Oxford: Blackwell Publishing.

Baumann-Pauly, D., Wickert, C., Spence, L. J., \& Scherer, A. G. (2013). Organizing Corporate Social Responsibility in Small and Large firms: Size Matters. Journal of Business Ethics, 115(4), 693-705.

Baumol, W. J. (1990). Entrepreneurship: Productive, Unproductive and Destructive. Journal of Political Economy, 98(1), 893-921.

Beadle, R., \& Moore, G. (2006). MacIntyre on Virtue and Organization. Organization Studies, 27(3), 323-340.

Bentham, J. (1789/2007). An Introduction to the Principles of Morals and legislation. Oxford: Dover Philosophical Classics.

Berger, P., \& Luckman, T.L. (1966). The social construction of reality: A treatise in the sociology of knowledge. New York, NY: Doubleday.

Berle, A., \& Means, G. (1932). The Modern Corporation and Private Property. New York: Macmillan.

Besser, T. L. (2012). The consequences of social responsibility for small business owners in small towns. Business Ethics: A European review, 21(2), 129-139.

Besser, T. L., \& Miller, N. J. (2001). Is the good corporation dead? The community social responsibility of small business operators. The Journal of Socio-Economics, $30(3), 221-241$. 
Besser, T. L., \& Miller, N. J. (2004). The Risk of Enlightened Self-Interest: Small Businesses and Support for Community. Business \& Society, 43(4), 398-425.

Besser, T. L., Miller, N., \& Perkins, R. K. (2006). For the greater good: business networks and business social responsibility to communities. Entrepreneurship and Regional Development, 18(4), 321-339.

Biondi, V., Iraldo, F., \& Meredith, S. (2002). Achieving sustainability through environmental innovation: the role of SMEs. International Journal of Technology Management, 24(5-6), 612-626.

Blackburn, M., \& McGhee, P. (2007). The Excellent Entrepreneur: Old Virtues for New Ventures? Australian Journal of Professional and Applied Ethics, 9(2), 74-89.

Blackburn, R., \& Stokes, D. (2000). Breaking Down the Barriers: Using Focus Groups to Research Small and Medium-sized Enterprises. International Small Business Journal: Researching Entrepreneurship, 19(1), 44-67.

Blombäck, A., \& Wigren, C. (2009). Challenging the importance of size as determinant for CSR activities. Management of Environmental Quality: An International Journal, 20(3), 255-270.

Blundel, R., Spence, L. J., \& Zerbinati, S. (2010). Entrepreneurial Social Responsibility: Scoping the Territory. In L. J. Spence \& M. Painter-Morland (Eds.), Ethics in Small and Medium Sized Enterprises: A global commentary (pp. 123-145), International Society for Business, Economics and Ethics Book Series (Vol 2). Dordrecht, NL: Springer.

Bolton, J. E. (1971). Report of the Committee of Enquiry on Small Firms. London: HMSO.

Bordieu, P. (1986). The Forms of Capital. In J. G. Richardson (Ed.), Handbook of theory and Research for the Sociology of Education (pp. 241-258). New York, NY: Greenwood Press. 
Bordieu, P., \& Wacquant, L. J. (1992). An invitation to reflexive sociology. Cambridge: Cambridge University Press.

Borgerson, J. (2007). On the Harmony of Feminist Ethics and Business Ethics. Business and Society Review, 112(4), 477-509.

Bowen, H. R. (1953). Social Responsibilities of the Businessman. New York, NY: Harper and Row.

Brammer, S., \& Millington, A. (2004). Stakeholder Pressure, Organizational Size, and the Allocation of Departmental Responsibility for the Management of Corporate Charitable Giving. Business and Society, 43(3), 268-295.

Brenkert, G. (2009). Innovation, rule breaking and ethics of entrepreneurship. Journal of Business Venturing, 24(5), 448-464.

Brønn, P. S., \& Vidaver-Cohen, D. (2009). Corporate Motives for Social Initiative: Legitimacy, Sustainability, or the bottom line? Journal of Business Ethics, 87(1), 91109.

Brooks, S. B. (2005). Corporate social responsibility and strategic management: the prospects for converging discourses. Strategic Change, 14(7), 401-411.

Brooks, S. B. (2008). Economic rationality, embeddedness and community: A critical study of corporate social responsibility among small to medium sized enterprises (Unpublished doctoral dissertation). Cardiff University, Cardiff.

Brooks, S. B. (2010). CSR and the straight-jacket of economic rationality. International Journal of Sociology and Social Policy, 30(11/12), 604-617.

Brown, D. J., \& King, J. B. (1982). Small business ethics: Influences and perceptions. Journal of Small Business Management, 20(1), 11-18. 
Bryman, A., \& Bell, E. (2011). Business Research Methods ( $3^{\text {rd }}$ ed.). New York, NY: Oxford University Press.

Bucar, B., \& Hisrich, R. D. (2001). Ethics of managers vs. entrepreneurs. Journal of Development Entrepreneurship, 6(1), 59-82.

Bucar, B., Glas, M., \& Hisrich, R. D. (2003). Ethics and entrepreneurs: An international comparative study. Journal of Business Venturing, 18(2), 261-281.

Buccholz, R. A. (1991). Corporate Responsibility and the Good Society: From Economics to Ecology. Business Horizons, 34(4), 19-31.

Buchholz, R. A., \& Rosenthal, S. B. (2005). Toward a Contemporary Conceptual Framework for Stakeholder Theory. Journal of Business Ethics, 58(1-3), 137-148.

Burr, V. (2003). Social Constructionism (2 ${ }^{\text {nd }}$ ed.). London: Routledge.

Burr, V. (2015). Social Constructionism ( $3^{\text {rd }}$ ed.). London: Routledge.

Burrell, G., \& Morgan, G. (1979). Sociological Paradigms and Organisational Analysis. Aldershot, Gower Publishing Company.

Burton, B. K., \& Goldsby, M. (2009). Corporate Social Responsibility Orientation, Goals and Behavior: A Study of Small Business Owners. Business and Society, 48(1), 88-104.

Campbell, J. L. (2007). Why Would Corporations Behave in Socially Responsible Ways? An Institutional Theory of Corporate Social Responsibility. The Academy of Management Review, 32(3), 946-967.

Campin, S., Barraket, J., \& Luke, B. (2013). micro-Business Community Responsibility in Australia: Approaches, Motivations and Barriers. Journal of Business Ethics, 115(3), 489-513. (DOI 10.1007/s10551-012-1396-1) 
Campopiano, G., De Massis, A., \& Cassia, L. (2012). The Relationship between Motivations and Actions in Corporate Social Responsibility: An Exploratory Study. International Journal of Business and Society, 13(3), 391-425.

Carr, A. (1968). Is Business Bluffing Ethical? Harvard Business Review, 46, 143 153.

Carroll, A. B. (1979). A Three-Dimensional Conceptual Model of Corporate Performance. Academy of Management Review, 4(4), 497-505.

Carroll, A. B. (1991). The pyramid of corporate social responsibility: Toward the moral management of organizational stakeholders. Business Horizons, 34(4), 39-48.

Carroll, A. B. (1999). Corporate Social Responsibility: Evolution of a Definitional Construct. Business \& Society, 38(3), 268-295.

Carroll, A. (2008). A History of Corporate Social Responsibility Concepts and Practises. In A. Crane, D. Matten, A. McWilliams, J. Moon \& S. Siegal (Eds.), The Oxford Handbook of Corporate Social Responsibility (pp. 19-46). Oxford: Oxford University Press.

Carroll, A. B. (2016). Carroll's pyramid of CSR: taking another look. International Journal of Corporate Social Responsibility, 1(1), 3.

Carroll, A. B., \& Buchholtz, A. (2014). Business \& Society: Ethics, Sustainability, and Stakeholder Management $\left(9^{\text {th }}\right.$ ed.). Stamford, CT: Cengage Learning.

Carroll, A. B., \& Shabana, K. M. (2010). The Business Case for Corporate Social Responsibility: A Review of Concepts, Research and Practice. International Journal of Management Reviews, 12(1), 85-105.

Carson, D., Gilmore, A., Perry, C., \& Gronhaug, K. (2001). Qualitative Marketing Research. London: Sage. 
Cassells, S., \& Lewis, K. (2011). SMEs and environmental responsibility: do actions reflect attitudes? Corporate Social Responsibility and Environmental Management, 18(3), 186-199.

Castka, P., Balzarova, M. A., Bamber, C. J., \& Sharp, J. M. (2004). How can SMEs effectively implement the CSR agenda? A UK case study perspective. Corporate Social Responsibility and Environmental Management, 11(3), 140-149.

Chrisman, J. J., \& Archer, R. W. (1984). Small Business Social Responsibility: Some Perceptions and insights. American Journal of Small Business, 9(2), 46-58.

Chrisman, J. J., \& Fry, F. L. (1982). Public versus business expectations: Two views on social responsibility for small business. Journal of Small Business Management, 20(1), 19-26.

Chrisman, J. J., Chua, A., Pearson., \& Barnett, T. (2012). Family involvement, family influence, and family-centred non-economic goals in small firms. Entrepreneurship: Theory and Practice, 36(2), 267-293.

Christopher, D. A. (2003). Small business pilfering: the "trusted" employee(s). Business Ethics: A European Review, 12(3), 284-297.

Ciliberti, F., Baden, D., Harwood, I. A. (2009). Insights into supply chain pressure on CSR-practice: A multiple-case study of SMEs in the UK. Operations and Supply Chain Management, 2(3), 154-166.

Clark, J. M. (1939). Social Control of Business. New York: McGraw-Hill.

Clarke, J., \& Holt, R. (2010). Reflective Judgement: Understanding Entrepreneurship as Ethical Practice. Journal of Business Ethics, 94(3), 317-331.

Colucci, E. (2007). "Focus groups can be fun": The use of activity-oriented questions in focus group discussions. Qualitative Health Research, 17(10), 14221433. 
Committee for Economic Development. (1971). Social responsibilities of business corporations. New York, NY: Committee for Economic Development.

Commission of the European Communities (2005). Implementing the Community Lisbon Programme Modern SME Policy for Growth and Employment. (COM, 551/F1). Retrieved from http://ec.europa.eu/transparency/regdoc/rep/1/2005/EN/12005-551-EN-F1-1.Pdf accessed on 17 August 2017.

Corbin, J. M., \& Strauss, A. L. (2008). Basics of Qualitative research: Techniques and Procedures for Developing Grounded Theory ( $3^{\text {rd }}$ ed.). Thousand Oaks, CA: Sage Publications.

Cornelissen, J. P. (2017). Preserving Theoretical Divergence in Management Research: Why the Explanatory Potential of Qualitative Research Should Be Harnessed Rather than Suppressed. Journal of Management Studies, 54(3), 368-383.

Courrent, J. M., \& Gundolf, K. (2009). Proximity and Micro-Enterprise Manager's Ethics: A French Empirical Study of Responsible Business Attitude. Journal of Business Ethics, 88(4), 749-762.

Crane, A., \& Matten, D. (2010). Business Ethics: Managing Corporate Citizenship and Sustainability in the Age of Globalization (3rd ed.). Oxford: Oxford University Press.

Crane, A., \& Matten, D. (2016). Business Ethics: Managing Corporate Citizenship and Sustainability in the Age of Globalization (4 ${ }^{\text {th }}$ ed.). Oxford: Oxford University Press.

Crane, A., Mcwilliams, A., Matten, D., Moon, J., \& Siegal, D. (2008). The Oxford Handbook of Corporate Social Responsibility. Oxford: Oxford University Press.

Crane, A., Palazzo, G., Spence, L. J., \& Matten, D. (2014). Contesting the Value of “Creating Shared Value”. California Management Review, 56(2), 130-153. 
Crotty, M. (1998). The Foundations of Research: Meaning and Perspectives of Social Research. New South Wales: Allen and Unwin.

Curran, J., \& Blackburn, R. A. (1994). Small firms and local economic networks: The death of the local economy. London: Paul Chapman Publishing.

Curran, J., \& Blackburn, R. A. (2001). Researching the Small Enterprise. London: Sage Publications.

Curran, J., Rutherfoord, R., \& Smith, S. L. (2000). Is there a local business community? Explaining the non-participation of small business in local economic development. Local Economy, 15(2), 128-143.

Cyr, J. (2016). The Pitfalls and Promise of Focus Groups as a Data Collection Method. Sociological Methods \& Research, 45(2), 231-259.

D’Aprile, G., \& Mannarini, T. (2012). Corporate social responsibility: a psychosocial multidimensional construct. Journal of Global Responsibility, 3(1), 48-65.

Dandridge, T. C. (1979). Children Are Not "Little Grown-Ups": Small Business Needs Its Own Organizational Theory. Journal of Small Business Management, $17(2), 53-57$.

Davies, I. A., \& Crane, A. (2010). Corporate social responsibility in small-and medium-size enterprises: investigating employee engagement in fair trade companies. Business Ethics: A European Review, 19(2), 126-139.

Davis, K. (1960). Can Business Afford to Ignore Social Responsibilities? California Management Review, 2(3), 70-76.

Davis, K. (1973). The Case for and against Business Assumption of Social Responsibilities. The Academy of Management Journal, 16(2), 312-322. 
De Clercq, D., Danis, W. M., \& Dakhli, M. (2010). The moderating effect of institutional context on the relationship between associational activity and new business activity in emerging economies. International Business Review, 19(1), 85101.

Dees, G., \& Starr. (1992). Entrepreneurship through an ethical lens: Dilemmas and issues for research and practice (pp. 89-116). In D. L. Sexton \& J. D. Karsada (Eds.), The State of the Art of Entrepreneurship. Boston, MA: PWS Publishing.

Deetz, S. (1994). Future of the Discipline: The Challenges, the Research, and the Social Contribution. In S. Deetz (Ed.). Communication Yearbook 17 (pp. 565-600). Newbury Park, CA: Sage.

Deetz, S. (1996). Describing Differences in Approaches to Organizational Science: Rethinking Burrell and Morgan and Their Legacy. Organisation Science, 7(2), 191207.

Del Baldo, M. (2012). Corporate social responsibility and corporate governance in Italian SMEs: the experience of some "spirited businesses". Journal of Management \& Governance, 16(1), 1-36.

Demuijnck, G., \& Ngnodjom, H. (2013). Responsibility and Informal CSR in Formal Cameroonian SMEs. Journal of Business Ethics, 112(4), 653-665.

Department for Business, Energy \& Industrial Strategy (2017). Business Population Estimates for the UK and Regions 2017. Retrieved from https://assets.publishing.service.gov.uk/government/uploads/system/uploads/attachm ent_data/file/663235/bpe_2017_statistical_release.pdf accessed 23 July 2018.

Department for Business Innovation and Skills. (2014). Business Population Estimates for the $U K$ and Regions 2014. (URN 14/92). Retrieved from https://www.gov.uk/government/uploads/system/uploads/attachment_data/file/37793 4/bpe_2014_statistical_release.pdf accessed 23 July 2018. 
Donaldson, T., \& Dunfee, T. W. (1994). Toward a Unified Conception of Business Ethics: Integrative Social Contracts Theory. The Academy of Management Review, 19(2), 252-284.

Donia, M. B., Tetrault Sirsly, C. A., \& Ronen, S. (2017). Employee Attributions of Corporate Social Responsibility as Substantive or Symbolic: Validation of a Measure. Applied Psychology, 66(1), 103-142.

Dunfee, T., Bowie, N., Hennessy, J., Nelson, K., \& Robertson, D. (1991). Firm size and employees attitudes about ethics: some preliminary empirical evidence. In B. Harvey, H. van Luijk \& and G. Corbetta (Eds.). Market Morality and Company Size (pp. 103-118). London: Kluwer.

Dunham, L. C. (2010). From Rational to Wise Action: Recasting Our Theories of Entrepreneurship. Journal of Business Ethics, 92(4), 513-530.

Duriau, V. J., Reger, R. K., \& Pfarrer, M. D. (2007). A Content Analysis of the Content Analysis Literature in Organization Studies: Research Themes, Data Sources, and Methodological Refinements. Organizational Research Methods, $10(1), 5-34$.

Durkheim, E. (1893/1964). The Division of Labor In Society. New York: Free Press.

Durkheim, E. (1893/1960). The Division of Labour in Society. Glencoe, Free Press.

Dyer, W. G., \& Handler, W. (1994). Entrepreneurship and family business: Exploring the connections. Entrepreneurship Theory and Practice, 19(1), 71-83.

Eakin, J. M., \& MacEachen, E. (1998). Health and the Social Relations of Work: A Study of the Health-related Experiences of Employees in Small Workplaces. Sociology of Health and Illness, 20(6), 896-914. 
Easterby-Smith, M., Thorpe, R., \& Lowe, A. (2002). Management Research: An Introduction ( $2^{\text {nd }}$ ed.). London: Sage Publications.

Easterby-Smith, M., Thorpe, R., \& Jackson, P. R. (2012). Management Research (4 ${ }^{\text {th }}$ ed.). London: Sage Publications.

Eberstadt, N. (1973). What History Tells us about Corporate Responsibilities. Business and Society Review, 7, 76-81.

Epstein, E. (1987). The Corporate Social Policy Process: Beyond Business Ethics, Corporate Social Responsibility, and Corporate Social Responsiveness. California Management Review, 29(3), 99-114.

Eriksson, P., \& Kovalainen, A. (2008). Qualitative Methods in Business Research. London: Sage Publications.

European Commission. (2015). User Guide to the SME Definition. Retrieved from http://ec.europa.eu/growth/tools-

databases/newsroom/cf/itemdetail.cfm?item_id $=8274 \&$ lang $=$ en\&title $=$ The $\% 2$ Drevis ed\%2Duser\%2Dguide\%2Dto\%2Dthe\%2DSME\%2Ddefinition retrieved 5 December 2016 .

European Commission. (2017). Entrepreneurship and Small and medium-sized enterprises (SMEs). Retrieved from http://ec.europa.eu/growth/smes/businessfriendly-environment/sme-definition/ accessed 23 July 2018.

European Commission. (2018). Corporate Social Responibiltiy (CSR). Retrieved from https://ec.europa.eu/growth/industry/corporate-social-responsibility_en accessed 23 July 2018.

Fassin, Y. (2008). SMEs and the fallacy of formalising CSR. Business Ethics: A European Review, 17(4), 363-378.

Fassin, Y., Van Rossem, A., \& Buelens, M. (2011). Small Business Owner- 
Managers' Perceptions of Business Ethics and CSR-Related Concepts. Journal of Business Ethics, 98(3), 425-453.

Federation of Small Businesses (FSB). (2011). Micro and Small Business in the EU: What's in it for you? Retrieved from http://www.fsb.org.uk/092/assets/whats-in-itfor-me\%20(2).pdf accessed 4 December 2015.

Federation of Small Businesses (FSB). (2017). What FSB does: Mission and Corporate Objectives. Retrieved from https://www.fsb.org.uk/about-us/about-the-fsb accessed 23 July 2018.

Fenwick, T. (2010). Learning to practice social responsibility in small business: challenges and conflicts. Journal of Global Responsibility, 1(1), 149-169.

Fevre, R. (2000). The Demoralization of Western Culture: Social Theory and the Dilemmas of Modern Living. London: Continuum.

Fevre, R. (2003). The New Sociology of Economic Behaviour. London: Sage.

Fisher, L., \& A, Lovell. (2009). Business Ethics and Values: Individual Corporate and International Perspectives $\left(3^{\text {rd }}\right.$ ed.). Essex: Pearson Education Limited.

Fisher, K., Geenen, J., Jurcevic, M., McClintock, K., \& Davis, G. (2008). Applying asset-based community development as a strategy for CSR: a Canadian perspective on a win-win for stakeholders and SMEs. Business Ethics: A European Review, 18(1), 66-82.

Fraj-Andrés, E., López-Pérez, M. E., Melero-Polo, I., \& Vázquez-Carrasco, R. (2012). Company image and corporate social responsibility: reflecting with SMEs' managers. Marketing Intelligence and Planning, 30(2), 266-280.

Frederick, W. (2006). Corporation Be Good: The Story of Corporate Social Responsibility. Indianapolis, IN: Dog Ear publishing. 
Frederiksen, C. S. (2010). The Relation Between Policies Concerning Corporate Social Responsibility (CSR) and Philosophical Moral Theories - An Empirical Investigation. Journal of Business Ethics, 93(3), 357-371.

Freeman, E. (1984). Strategic Management: A Stakeholder Approach. Boston, MA: Pitman.

Freeman, R. E., \& Liedtka, J. (1991). Corporate Social Responsibility: A Critical Approach. Business Horizons, 34(4), 92-98.

Friedman, M. (1970, September 13). The Social Responsibility of Business is to Increase its Profits. New York Times Magazine, 13, 32-33, 122-126.

Fritzsche, D. J., \& Becker, H. (1984). Linking Management Behavior to Ethical Philosophy - An Empirical Investigation. The Academy of Management Journal, 27(1), 166-175.

Fuller, T., \& Tian, Y. (2006). Social and Symbolic Capital and Responsible Entrepreneurship: An Empirical Investigation of SME Narratives. Journal of Business Ethics, 67(3), 287-304.

Gallo, P. J., \& Christensen, L. J. (2011). Firm Size Matters: An Empirical Investigation of Organzational Size and Ownership on Sustainability-Related Behaviours. Business and Society, 50(2), 315-349.

Gamson, W.A. (1992). Talking Politics. New York, NY: Cambridge University Press.

Garay, L., \& Font, X. (2012). Doing good to do well? Corporate social responsibility reasons, practices and impacts in small and medium accommodation enterprises. International Journal of Hospitality Management, 31(2), 329-337.

Garriga, E., \& Melé, D. (2004). Corporate Social Responsibility Theories: Mapping the Territory. Journal of Business Ethics, 53(1-2), 51-71. 
Geertz, C. (1973). Thick Description: Toward an Interpretive Theory of Culture. In C. Geertz (Ed.), The Interpretation of Cultures. New York, NY: Basic Books.

Gelbmann, U. (2010). Establishing strategic CSR in SMEs: an Austrian CSR quality seal to substantiate the strategic CSR performance. Sustainable Development, 18(2), 90-98.

Gephart, R. P. (1991). Multiple methods for tracking corporate social performance: Insights from a study of major industrial accidents. In J. E. Post (ed.), Research in Corporate Social Performance and Policy (Vol. 12) (pp. 359-385). Greenwich, CT: JAI Press.

Gephart, R. P. (1993). The textual approach: Risk and blame in disaster sensemaking. Academy of Management Journal 36(6), 1465-1514.

Giddens, A. (1987). Social Theory and Modern Sociology. California, CA: Stanford University Press.

Gilligan, C. (1982). In a Different Voice: Psychological Theory and Women's Development. Cambridge, MA: Harvard University Press.

Girod-Séville, M., \& Perret, V. (2001) Epistemological Foundations. In R. A. Thiétart (Ed.), Doing Management Research: A Comprehensive Guide, (pp. 13-30). London: Sage Publications.

Goffman, E. (1974). Frame Analysis: An Essay on the Organization of Experience. Cambridge, MA: Harvard University Press.

Graafland, J., Mazereeuw, C., \& Yahia, A. (2006). Islam and Socially Responsible Business Conduct: An Empirical Study of Dutch Entrepreneurs. Business Ethics: A European Review, 15(4), 390-406.

Graafland, J., Van de Ven, B., \& Stoffele, N. (2003). Strategies and Instruments for 
Organising CSR by Small and Large Businesses in the Netherlands. Journal of Business Ethics, 47(1), 45-60.

Granerud, L. (2011). Social responsibility as an intermediary for health and safety in small firms. International Journal of Workplace Health Management, 4(2), 10301039.

Granovetter, M. (1985). Economic Action and Social Structure: The Problem of Embeddedness. American Journal of Sociology, 91(3), 481-510.

Guba, E. G., \& Lincoln, Y. S. (1994). Competing Paradigms in Qualitative Research. In N. K. Denzin \& Y. S. Lincoln (Eds.), Handbook of Qualitative Research (pp. 105-117). Thousand Oaks, CA: Sage Publications.

Gupta, S., \& Kalra, N. (2012). Impact of Corporate Social Responsibility on SMEs in India. Asia-Pacific Journal of Management Research and Innovation, 8(2), 133143.

Habermas, J. (1983). Moral Consciousness and Communicative Action. Cambridge, MA: Massachusetts Institute of Technology.

Hansen, A., Cottle, S., Negrine, R., \& Newbold, C. (1998). Content Analysis. In S. Cottle, A. Hansen, C. Newbold \& R. Negrine (Eds.), Mass Communication Research Methods (pp. 91-129). Basingstoke: Palgrave Macmillan.

Harvey, B., van Luijk, H., \& Corbetta, G. (1991). Market Morality and Company Size. London: Kluwer.

Hamman, R., Agbazue, T., Kapelus, P., \& Hein, A. (2005). Universalizing Corporate Social Responsibility? South African Challenges to the International Organization for Standardization's New Social Responsibility Standard. Business and Society Review, 110(1), 1-19.

Hammann, E. M., Habisch, A., Pechlaner, H. (2009). Values that create value: 
socially responsible business practices in SMEs- empirical evidence from German companies. Business Ethics: A European Review, 18(1), 37-51.

Hannafey, F. T. (2003). Entrepreneurship and Ethics: A Literature Review. Journal of Business Ethics, 46(2), 99-110.

Held, V. (1990). Feminist transformations of moral theory. Philosophy and Phenomenological Research, 50, 321-344.

Held, V. (2006). The Ethics of Care: Personal, Political and Global. Oxford: Oxford University Press.

Hofstede, G. H. (1984). Culture's Consequences: International Differences in Work Related Values. Beverly Hills, CA: Sage Publications.

Holloway, I. (1997). Basic Concepts for Qualitative Research. Oxford: Blackwell Science.

Holliday, R. (1995). Investigating small firms: Nice work? London: Routledge.

Hornsby, J. S., Kuratko, D. F., Naffziger, D. W., LaFollette, W. R., and Hodgetts, R. M. (1994). The ethical perceptions of small business owners: A factor analytic study. Journal of Small Business Management, 32(4), 9-16.

Hsieh, H. F., \& Shannon, S. E. (2005). Three Approaches to Qualitative Content Analysis. Qualitative Health Research, 15(9), 1277-1288.

Hudson, L. A., \& Ozanne, J. L. (1988). Alternative Ways of Seeking Knowledge in Consumer Research. Journal of Consumer Research, 14(4), 508-521.

Huff, A. S. (1990). Mapping strategic thought. Chichester, NY: Wiley.

Humphreys, N., Robin, D., Reidenbach, R. and Moak, D. (1993). The ethical decision making process of small business owner/managers and their customers. 
Journal of Small Business Management, 31(3), 9-2.

Ibrahim, S. (2014). Corporate Social Responsibility in Small and Medium-Sized Enterprises: A Developing Country Perspective. (Doctoral dissertation). Retrieved from the British Library Board e-theses online service. (uk.bl.ethos.655350)

Ibrahim, S., Jamali, D., \& Karatas-Ozkan, M. (2012). Corporate Social Responsibility (CSR) in Small and Medium-Sized Enterprises: A Developing Country Perspective. In S. Underwood \& R. Blundel (Eds.), Social and Sustainable Enterprise: Changing the Nature of Business (pp. 167-192). Bradford: Emerald Group Publishing.

International Trade Centre. (2015). SMEs Competitiveness Outlook 2015: Connect, Compete and Change for Inclusive Growth. Geneva: International Trade Centre.

Inyang, B. J. (2013). Defining the Role of Engagement of Small and Medium-Sized Enterprises (SMEs) in Corporate Social Responsibility (CSR). International Business Research, 6(5), 123-132.

Jaffe, E. D., \& Pasternak, H. (2006). Moral intensity as a predictor of social responsibility. Business Ethics: A European Review, 15(1), 53-63.

Jamali, D., Zanhour, M., \& Keshishian, T. (2009). Peculiar Strengths and Relational Attributes of SMEs in the Context of CSR. Journal of Business Ethics, 87(3), 355377.

Jenkins, H. (2004). A Critique of Conventional CSR Theory: An SME Perspective. Journal of General Management, 29(4): 37-57.

Jenkins, H. (2006). Small Business Champions for Corporate Social Responsibility. Journal of Business Ethics, 67(3), 241-256.

Johnson, H. L. (1971). Business in Contemporary Society: Framework and Issues. Belmont, CA: Wadsworth. 
Jones, T. (1991). Ethical Decision Making by Individuals in Organizations: An Issue-Contingent Model. Academy of Management Review, 16(2), 366-395.

Jones, C., Parker, M., \& ten Bos, R. (2005). For Business Ethics. Abingdon: Routledge.

Jones, M., Marshall, S., \& Mitchell, R. (2007). Corporate Social Responsibility and the Management of Labour in Two Australian Mining Industry Companies. Corporate Governance: An International Review, 15(1), 57-67.

Jones, C., \& Spicer, A. (2005). The Sublime Object of Entrepreneurship. Organization, 12(2), 223-246.

Kalberg, S. (1980). Max Weber's types of rationality: Cornerstones for the analysis of rationalization processes in history. American Journal of Sociology, 85(5), 11451179 .

Kang, Y. C. (1995). Before-Profit CSR. Stakeholder Capitalism and Just Enterprise System (Doctoral Dissertation). $\quad$ Retrieved from https://search.proquest.com/docview/304218512/?pq-origsite=primo (9614201)

Kang, Y. C., \& Wood, D. J. (1995). Before Profit Social Responsibility: Turning the Economic Paradigm Upside down. In IABS, 1995: Proceedings of the 6th Annual Meeting of the International Association for Business and Society (Vol. 6, p. 418).

Kant, I. (1781/1998). Critique of Pure Reason. (P. Guyer \& A. Wood, Trans.). Cambridge University Press. (Original work published 1781)

Kant, I. (1788/1997). Critique of Practical Reason. (M. Gregor, Trans.). Cambridge, Cambridge University Press. (Original work published in 1788)

Kechiche, A., \& Soparnot, R. (2012). CSR within SMEs: Literature Review. International Business Research, 5(7), 97. 
Killinger, B. (2010). Integrity: Doing the Right Thing for the Right Reason. Canada: McGill-Queen's University Press.

Knights, D., \& Wilmott, H. (1989). Power and Subjectivity at Work: From Degradation to Subjugation in Social Relations. Sociology, 23(4), 535-558.

Kohlberg, L. (1984). The Psychology of moral development: the nature and validity of moral stages. San Francisco: Harper Row.

Koos, S. (2012). The institutional embeddedness of social responsibility: a multilevel analysis of smaller firms' civic engagement in Western Europe. SocioEconomic Review, 10(1), 135-162.

Kreps, T. (1940). Measurement of the Social Performance of Business. An investigation of concentration of economic power for the temporary national economic committee [Monograph 7]. Washington, WA: US Government Printing Office.

Krippendorff, K. (1980). Content Analysis: An Introduction to Its Methodology. Newbury Park, CA: Sage Publications.

Lähdesmäki, M. (2005). When Ethics Matters: Interpreting the Ethical Discourse of Small Nature-Based Entrepreneurs. Journal of Business Ethics, 61(1), 55-68.

Lähdesmäki, M. (2012). Construction of owner-manager identity in corporate social responsibility discourse. Business Ethics: A European Review, 2(2), 129-237.

Lähdesmäki, M., Siltaoja, M., \& Spence, L. J. (2017). Stakeholder Salience for Small Businesses: A Social Proximity Perspective. Journal of Business Ethics, 1-13.

Lähdesmäki, M., \& Suutari, T. (2012). Keeping at Arm's Length or Searching for Social Proximity? Corporate Social Responsibility as a Reciprocal Process Between Small Businesses and the Local Community. Journal of Business Ethics, 108(4), 
481-493.

Lähdesmäki, M., \& Takala, T. (2012). Altruism in business - an empirical study of philanthropy in the small business context. Social Responsibility Journal, 8(3), 373388.

Lancaster, G. (2005). Research Methods in Management: A concise introduction to research in management and business consultancy. Oxford: Elsevier.

Lange, D., \& Washburn, N. T. (2012). Understanding Attributions of Corporate Social Irresponsibility. Academy of Management Review, 37(2), 300-326.

Lantos, G. P. (2001). The Boundaries of Strategic Corporate Social Responsibility. Journal of Consumer Marketing, 18(7), 595-632.

LeCompte, M. D., \& Goetz, J. P. (1982). Problems of Reliability and Validity in Ethnographic Research. Review of Educational Research, 52(1), 31-60.

Lee, M. D. P. (2008). A review of the theories of corporate social responsibility: It's evolutionary path and the road ahead. International Journal of Management Reviews, 10(1), 53-73.

Lee, M. H., Mak, A. K., \& Pang, A. (2012). Bridging the Gap: An Exploratory Study of Corporate Social Responsibility among SMEs in Singapore. Journal of Public Relations Research, 24(4), 299-317.

Lepoutre, J., \& Heene, A. (2006). Investigating the Impact of Firm Size on Small Business Social Responsibility: A Critical Review. Journal of Business Ethics, 67(3), 257-273.

Levin, D. M. (1988) The Opening of Vision: Nihilism and the Postmodern Situation. London: Routledge

Levinas, E. (1961/1969). Totality and Infinity: An Essay on Exteriority. Pittsburgh, 
PA: Duquesne University Press.

Levitt, T. (1958). The Dangers Of Social Responsibility. Harvard Business Review, 36(5), 41-50.

Liedtka, J. M. (1992). Exploring Ethical Issues Using Personal Interviews. Business Ethics Quarterly, 2(2), 161-181.

Liedtka, J. (1996). Feminist morality and competitive reality: A role for an ethic of care? Business Ethics Quarterly, 6(2), 179-200.

Litz, R. A., \& Samu, S. (2008). Altruistic by Association, Altruistic for Advantage? Buying Groups and Small Firm Community Involvement. Nonprofit and Voluntary Sector Quarterly, 37(4), 646-667.

Liu, H., \& Fong, M. (2010). The Corporate Social Responsibility Orientation of Chinese Small and Medium Enterprises. Journal of Business Systems, Governance \& Ethics, 5(3), 33-70.

Longnecker, J., McKinney, J., \& Moore, C. (1988). Egoism and independence: Entrepreneurial ethics. Organizational Dynamics, 16(3), 64-72.

Longo, M., Mura, M., \& Bonoli, A. (2005). Corporate social responsibility and corporate performance: the case of Italian SMEs. The International Journal of Business in Society, 5(4), 28-42.

Luetkenhorst, W. (2004). Corporate social responsibility and the development agenda. Intereconomics, 39(3), 157-166.

Luken, R., \& Stares, R. (2005). Small business responsibility in developing countries: a threat or an opportunity? Business Strategy and the Environment, 14(1), $38-53$.

Lund-Thomsen, P., \& Pillay, R. G. (2012). CSR in industrial clusters: an overview 
of literature. The International Journal of Business in Society, 12(4), 568-578.

MacIntyre, A. (1981). After Virtue: A Study in Moral Theory. Notre Dame, IN: University of Notre Dame Press.

MacIntyre, A. (1985). After Virtue: A Study in Moral Theory (2 ${ }^{\text {nd }}$ ed.). London: Duckworth.

Marsh, D., \& Furlong, P. (2002). A Skin Not a Sweater: Ontology and Epistemology in Political Science. In D. Marsh \& S. Gerry (Eds.), Theory and Methods in Political Science (pp. 17-41). Basingstoke: Palgrave.

Massey, C. (2004). Employee practices in New Zealand SMEs. Employee Relations, 26(1), 94-105.

Matten, D., Crane, A., \& Chapple, W. (2003). Behind the mask: Revealing the true face of corporate citizenship. Journal of Business Ethics, 45(1-2), 109-120.

Maynard, M. (1994). Methods, Practice and Epistemology: The debate about Feminism and Research. In M. Maynard \& J. Purvis (Eds.), Researching Women's Lives from a Feminist Perspective (pp. 10-27). Abingdon: Taylor \& Francis.

McCracken, G. (1988). Qualitative Research Methods: The Long Interview (Vol. 13). Newbury Park, CA: Sage Publications.

McMahon, J. M., \& Harvey, R. J. (2007). The Effect of Moral Intensity on Ethical Judgement. Journal of Business Ethics, 72(4), 335-357.

McMylor, P. (2003). Moral Philosophy and Economic Sociology: What MacIntyre Learnt from Polanyi. International Review of Sociology, 13(2), 393-407.

McVea, J. F. (2009). A field study of entrepreneurial decision-making and moral imagination. Journal of Business Venturing, 24(5), 491-504. 
McWilliams, A., \& Siegel, D. (2001). Corporate Social Responsibility: A Theory of the Firm Perspective. Academy of Management Review, 26(1), 117-127.

McWilliams, A., Siegel, D. S., \& Wright, P. M. (2006). Corporate Social Responsibility: Strategic Implications. Journal of Management Studies, 43(1), 1-18.

Mencl, J., \& May, D. R. (2009). The Effects of Proximity and Empathy on Ethical Decision-Making: An Exploratory Investigation. Journal of Business Ethics, 85(2), 201-226.

Merton, R. K., Fiske, M., \& Kendall, P. (1990). The Focused Interview: A Manual of Problems and Procedures ( $2^{\text {nd }}$ ed.). New York, NY: Free Press.

Miles, M. B., \& Huberman, A. M. (1994). Qualitative data analysis: An expanded sourcebook. Thousand Oaks, CA: Sage Publications.

Mitchell, R. K., Agle, B. R., Chrisman, J. J., \& Spence, J. (2011). Toward a Theory of Stakeholder Salience in Family Firms. Business Ethics Quarterley, 21(2), 235255.

Mitchell, R. K., Agle, B. R., \& Wood, D. J. (1997). Toward a Theory of Stakeholder Identification and Salience: Defining the Principle of Who and What Really Counts. The Academy of Management Review, 22(4), 853-886.

Morgan, D. L. (1988). Focus Groups as Qualitative Research (Vol. 16). London: Sage publications.

Moore, G., Slack, R., \& Gibbon, J. (2009). Criteria for Responsible Business Practice in SMEs: An Exploratory Case of UK Fair Trade Organisations. Journal of Business Ethics, 89(2), 173-188.

Moore, G., \& Spence, L. (2006). Editorial: Responsibility and Small Business. Journal of Business Ethics, 67(3), 219-226. 
Morsing, M., \& Perrini, F. (2009). CSR in SMEs: do SMEs matter for the CSR agenda? Business Ethics: A European Review, 18(1), 1-6.

Munro, R. (2001). Calling for accounts: numbers, monsters and membership. The Sociological Review, 49(4), 473-493.

Murillo, D., \& Lozano, J. M. (2006). SMEs and CSR: An approach to CSR in their Own Words. Journal of Business Ethics, 67(3), 227-240.

Murillo, D., \& Lozano, J. M. (2009). Pushing forward SME CSR through a network: An account from the Catalan model. Business Ethics: A European Review, 18(1), 720.

Murphy, P.R., Smith, J. E., and Daley, J. M. (1992). Executive attitudes, organizational size and ethical issues: Perspectives on a service industry. Journal of Business Ethics, 11(1), 11-19. ,

Nejati, M., \& Ghasemi, S. (2012). Corporate social responsibility in Iran from the perspective of employees. Social Responsibility Journal, 8(4), 578-588

Nielsen, A. E., \& Thomson, C. (2009). Investigating CSR communication in SMEs: a case study among Danish middle managers. Business Ethics: A European Review, 18(1), 83-93.

Nijhof, A. H., \& Jeurissen, R. J. (2010). The glass ceiling of corporate social responsibility: Consequences of a business case approach towards CSR. International Journal of Sociology and Social policy, 30(11/12), 618-631.

Oppenheim, A. N. (2000). Questionnaire Design, Interviewing and Attitude Measurement, $\left(2^{\text {nd }}\right.$ ed. $)$. London: Bloomsbury Publishing.

Orlitzky, M., Schmidt, F. L., \& Rynes, S. L. (2003). Corporate Social and Financial Performance: A Meta-Analysis. Organization Studies, 24(3), 403-441. 
Patton, M. Q. (2002). Qualitative Research and Evaluation Methods (3 ${ }^{\text {rd }}$ ed.). London: Sage Publications.

Payne, D., \& Joyner, B. E. (2006). Successful U.S. Entrepreneurs: Identifying Ethical Decision-making and Social Responsibility Behaviors. Journal of Business Ethics, 65(3), 203-217.

Parker, M. (2002). Against management: organization in the age of managerialism. Cambridge: Polity Press.

Perrini, F. (2006). SMEs and CSR Theory: Evidence and Implications from an Italian Perspective. Journal of Business Ethics, 67(3), 305-316.

Polanyi, K. (1944/2001) The Great Transformation: The Political and Economic Origins of Our Time. Boston, MA: Beacon Press.

Porter, M. E., \& Kramer, M. R. (2002). The Competitive Advantage of Corporate Philanthropy. Harvard Business Review, 80(12), 56-68.

Porter, M. E., \& Kramer, M. R. (2006). The Link Between Competitive Advantage and Corporate Social Responsibility. Harvard Business Review, 84(12), 78-92.

Porter, M. E., \& Kramer, M. R. (2011). Creating Shared Value. Harvard Business Review, 89(1/2), 62-77.

Potter, J. (1996). Representing Reality: Discourse, Rhetoric and Social Construction. London: Sage.

Potter, J., \& Wetherell, M. (1987). Discourse and Social Psychology: Beyond Attitudes and Behaviour. London: Sage.

Premeaux, S. R. (2004). The Current Link Between Management Behavior and Ethical Philosophy. Journal of Business Ethics, 51(3), 269-278. 
Preuss, L., \& Perschke, M. J. (2010). Slipstreaming the Larger Boats: Social Responsibility in Medium-Sized Businesses. Journal of Business Ethics, 92(4), 531551.

Putnam, D. R. (2000). Bowling Alone: The Collapse and Revival of American Community. New York, NY: Simon and Schuster.

Quinn, J. J. (1997). Personal Ethics and Business Ethics: The Ethical Attitudes of Owner/Managers of Small Business. Journal of Business Ethics, 16(2), 119-127.

Rand, A. (1964). The Virtue of Selfishness: A New Concept of Egoism. New York: New American Library.

Rawls, J. (1971). A Theory of Justice. Cambridge, Massachusetts: Harvard University Press.

Rawlings, G. (2012). Intangible nodes and networks of influence: The ethics of tax compliance in Australian small and medium-sized enterprises. International Small Business Journal, 30(1), 84-95.

Rahim, M. M., \& Wisuttisak, P. (2013). Corporate Social Responsibility-Orientated Compliances and SMEs Access to Global Market: Evidence from Bangladesh. Journal of Asia-Pacific Business, 14(1), 58-83.

Reidenbach, R. E., \& Robin, D. P. (1990). Toward the Development of a Multidimensional Scale for Improving Evaluations of Business Ethics. Journal of Business Ethics, 9(8), 639-653.

Revell, A., Stokes, D., \& Chen, H. (2009). Small businesses and the environment: turning over a new leaf? Business Strategy and the Environment, 19(5), 273-288.

Robertson, D. (1993). Empiricism in business ethics: Suggested research directions. Journal of Business Ethics, 12(8), 585-599. 
Roche, B., \& Jakub, J. (2017). Completing Capitalism: Heal Business to Heal the World. Oakland, CA: Barrett-Koehler Publishers.

Rubin, H. J., \& Rubin, I. S. (2004). Qualitative Interviewing: The Art of Hearing Data $\left(2^{\text {nd }}\right.$ ed.). London: Sage Publications.

Russo, A., \& Perrini, F. (2010). Investigating Stakeholder Theory and Social Capital: CSR in Large Firms and SMEs. Journal of Business Ethics, 91(2), 207-221.

Russo, A., \& Tencati, A. (2009). Formal vs. informal CSR strategies: Evidence from Italian micro, small, medium-sized, and large firms. Journal of Business Ethics, 85(2), 339-353.

Rutherford, M. W., Buller, P. F., \& Stebbins, J. M. (2009). Ethical Considerations of the Legitimacy Lie. Entrepreneurship Theory and practice, 33(4), 949-964.

Ryan, M. K., David, B. \& Reynolds, K. J. (2004). Who Cares? The Effect of Gender and Context on the Self and Moral Reasoning. Psychology of Women Quarterly, $28(3), 246-255$.

Sachs, S., \& Ruehle, E. (2009). Sustainable Success with Stakeholders. The Untapped Potential. Basingstoke: Palgrave MacMillan.

Saldaña, J. (2015). The Coding Manual for Qualitative Researchers. London: Sage Publications.

Sarbutts, N. (2003). Can SMEs “do" CSR? A practitioner's view of the ways smalland medium- sized enterprises are able to manage reputation through corporate social responsibility. Journal of Communication Management, 7(4), 340-347.

Saunders, M., Lewis, P., and Thornhill, A. (2003). Research Methods for Business Students. ( $3^{\text {rd }}$ ed.). London: Prentice Hall.

Sayer, A. (2001). Reply to Holmwood. Sociology, 35(4), 967-984. 
Schaper, M. T., \& Savery, L. K. (2004). Entrepreneurship and Philanthropy: The Case of Small Australian Firms. Journal of Development Entrepreneurship, 9(3), 239-250.

Schlierer, H. J., Werner, A., Signori, S., Garriga, E., von Weltzien Hoivik, H., Van Rossem, A., \& Fassin, Y. (2012). How Do European SME Owner-Managers Make Sense of 'Stakeholder Management'?: Insights from a Cross-National Study. Journal of Business Ethics, 109(1), 39-51.

Schreck, P. (2011). Reviewing the Business Case for Corporate Social Responsibility: New Evidence and Analysis. Journal of Business Ethics, 103(2), 167-188.

Schwartz, M. S., \& Carroll, A. B. (2003). Corporate Social Responsibility: A ThreeDomain Approach. Business Ethics Quarterly, 13(4), 503-530.

Sen, S. (2011). Corporate social responsibility in small and medium enterprises: application of stakeholder theory and social capital theory (Doctoral dissertation). Retrieved from ePublications@SCU. (206).

Sen, S., \& Cowley, J. (2013). The Relevance of Stakeholder Theory and Social Capital Theory in the Context of CSR in SMEs: An Australian Perspective. Journal of Business Ethics, 118(2), 413-427.

Sethi, S. P. (1979). Dimensions of Corporate Social Performance: An Analytic Framework. California Management Review, 17(3), 58-64.

Schreck, P. (2011). Reviewing the Business Case for Corporate Social Responsibility: New Evidence and Analysis. Journal of Business Ethics, 103(2), 167-188.

Shapiro, G., \& Markoff, J. (1997). A Matter of Definiton. In C. W. Roberts (Ed.), Text Analysis for the Social Sciences: Methods for Drawing Statistical Inferences 
from Text and Transcripts (pp. 9-31). Mahwah, NJ: Lawrence Erlbaum Associates.

Singer, M. S. (1998). The role of subjective concerns and characteristics of the moral issue in moral considerations. British Journal of Psychology, 89(4), 663-679.

Southwell, C. (2004). Engaging SMEs in community and social issues. In L.J. Spence, A. Habisch, \& R. Schmidpeter (Eds.), Responsibility and social capital: The World of Small and Medium Sized Enterprises (pp. 96-111). Basingstoke: Palgrave Macmillan.

Spence, L. J. (1999). Does size matter? The state of the art in small business ethics. Business Ethics: A European Review, 8(3), 163-174.

Spence, L. J. (2004). Small Firm Accountability and Integrity. In G. Brenkert (Ed.), Corporate Integrity and Accountability (pp. 115-128). London: Sage.

Spence, L. J. (2007). CSR and Small Business in a European Policy Context: The Five "C" s of CSR and Small Business Research Agenda 2007. Business and Society review, 112(4), 533-552.

Spence, L. J. (2014). Business ethics and social responsibility in small firms. In E. Chell \& M. Karatas-Ozkan, (Eds.), Handbook of Research on Small Business and Entrepreneurship (pp. 1-22). Cheltenham, UK: Edward Elgar Publishing Limited.

Spence, L. J. (2016). Small Business Social Responsibility: Expanding Core CSR theory. Business and Society, 55(1), 23-55.

Spence, L. J., \& Lozano, J. F. (2000). Communicating about Ethics with Small Firms: Experiences from the U.K. and Spain. Journal of Business Ethics, 27(1-2), 43-53.

Spence, L. J., \& Painter-Morland, M. (Eds.). (2010). Ethics in Small and Medium Sized Enterprises: A Global Commentary. Dordrecht: Springer. 
Spence, L. J., \& Perrini, F. (2009). Practice and politics: Ethics and social responsibility in SMEs in the European Union. African Journal of Business Ethics, $4(2), 20-31$.

Spence, L. J., \& Perrini, F. (2011). Europe: Practice and politics: Ethics and social responsibility in SMEs in the European union. In L. Spence \& M. Painter-Morland (Eds.), Ethics in Small and Medium Sized Enterprises (pp. 35-54). Netherlands: Springer.

Spence, L. J., \& Rutherfoord, R. (2001). Social Responsibility, profit maximisation and the small firm owner-manager. Journal of Small Business and Enterprise Development, 8(2), 126-139.

Spence, L. J., \& Rutherfoord, R. (2003). Small Business and Empirical Perspectives in Business Ethics: Editorial. Journal of Business Ethics, 47(1), 1-5.

Spence, L. J., \& Schmidpeter, R. (2003). SMEs, Social Capital and the Common Good. Journal of Business Ethics, 45(1-2), 93-108.

Spence, L. J., Schmidpeter, R., \& Habisch, A. (2003). Assessing Social Capital: Small and Medium Sized Enterprises in Germany and the UK. Journal of Business ethics, 47(1), 17-29.

Smith, A. (1759/1976). The Theory of Moral Sentiments. D. D. Raphael \& A. L. Macfie (Eds.). Oxford: Clarendon Press.

Smith, A. (1776/1994). The Wealth of Nations. E. Cannan (Ed.). New York, NY: Modern Library.

Statistics for Wales. (2017). Size Analysis of Active Businesses in Wales, 2017 (SFR 189/2017). Retrieved from https:/gov.wales/docs/statistics/2017/171221-sizeanalysis-welsh-business-2017-en.pdf accessed on 18 July 2017.

Steiner, G. A. (1971). Business and Society. New York, NY: Random House. 
Stewart, D. W., \& Shamdasani, P.N. (1990). Applied Social Research Methods Series (Vol. 20). Focus Groups: Theory and Practice. California, CA: Sage Publications

Strauss, A. (1987). Qualitative Analysis for Social Scientists. New York, NY: Cambridge University Press.

Strauss, A., \& Corbin, J. (1998). Basics of Qualitative Researc ( $2^{\text {nd }}$ ed.). Thousand Oakes, CA: Sage Publications.

Swart, J. (1976). Profits Versus Social Responsibility: A Critical Analysis of Corporation Objectives from a Perspective of Communications Theory. In $A O M$, 1976: Proceedings of the $36^{\text {th }}$ Academy of Management Meeting (Vol. 1, pp. 320323). Kansas City, KS.

Sweeny, L. (2007). Corporate social responsibility in Ireland: barriers and opportunities experienced by SMEs when undertaking CSR. The International Journal of Business in Society, 7(4), 516-523.

Tang, Z., \& Tang, J. (2012). Stakeholder-firm power difference, stakeholders' CSR orientation, and SMEs' environmental performance in China. Journal of Business Venturing, 27(4), 436-455.

Tang, Z., Hull, C. E., \& Rothenberg, S. (2012). How Corporate Social Responsibility Engagement Strategy Moderates the CSR-Financial Performance Relationship. Journal of Management Studies, 49(7), 1274-1303.

Tantalo, C., Caroli, M. G., \& Vanevenhoven, J. (2012). Corporate social responsibility and SME's competitiveness. International Journal of Technology Management, 58(1/2), 129-151.

Thomas, D. R. (2006). A General Inductive Approach for Analyzing Qualitative Evaluation Data. American Journal of Evaluation, 27(2), 237-246. 
Thomas, W, I., \& Thomas, D. S. (1928). The Child in America: Behavior Problems and Programs. New York: Knopf.

Thompson, J. K., \& Smith, H. L. (1991). Social Responsibility and Small Business: Suggestions for Research. Journal of Small Business Management, 29(1), 30.

Thompson, J. K., Smith, H. L., \& Hood, J. N. (1993). Charitable Contributions by Small Businesses. Journal of Small Business Management, 31(3), 35-51.

Tilley, F. (2000). Small firm environmental ethics: how deep do they go? Business Ethics: A European Review, 9(1), 31-41.

Torugsa, N. A., O’Donohue, W., \& Hecker, R. (2013). Proactive CSR: An Empirical Analysis of the Role of its Economic, Social and Environmental Dimensions on the Association between Capabilities and Performance. Journal of Business Ethics, $115(2), 383-402$.

Udayasankar, K. (2008). Corporate Social Responsibility and Firm Size. Journal of Business Ethics, 83(2), 167-175.

Uhlaner, L. M., Berent-Braun, M. M., Jeurissen, R. J., \& de Wit, G. (2012). Beyond Size: Predicting Engagement in Environmental Management Practices of Dutch SMEs. Journal of Business Ethics, 109(4), 411-429.

Ullmann, A. E. (1985). Data in Search of a Theory: A Critical Examination of the Relationship among Social Performance, Social Disclosure, and Economic Performance of U.S. Firms. Academy of Management Review, 10(3), 540-557.

Vázquez-Carrasco, R., \& López-Pérez, M. E. (2013). Small \& medium-sized enterprises and Corporate Social Responsibility: a systematic review of the literature. Quality \& Quantity, 47(6), 3205-3218. 
Vives, A. (2006). Social and Environmental Responsibility in Small and Medium Enterprises in Latin America. The Journal of Corporate Citizenship, 21, 39-50.

Vogel, D. (1992). The Globalization of Business Ethics: Why America Remains Distinctive. California Management Review, 35(1), 30-49.

von Weltzien Hoivik, H., \& Melé, D. (2009). Can an SME become a global corporate citizen? Evidence from a case study. Journal of Business Ethics, 88(3), 551-563.

von Weltzien Hoivik, H., \& Shankar, D. (2011). How Can SMEs in a Cluster Repsond to Global Demands for Corporate Responsibility? Journal of Business Ethics, 101(2), 175-195.

Wartick, S. L., \& Cochran, P. L. (1985). The Evolution of the corporate Social Performance Model. The Academy of Management Review, 11(4), 758-769.

Webb, J. W., Tihanyi, L., Ireland, R. D., \& Sirmon, D. G. (2009). You Say Illegal, I Say Legitimate: Entrepreneurship in the Informal Economy. The Academy of Management Review, 34(3), 492-510.

Weber, M. (1930/2003). The Protestant Ethic and the Spirit of Capitalism. (T. Parsons, Trans.). Abingdon: Routledge. (Original work published 1930)

Weber, M. (1978). The Technical Superiority of Bureaucratic Organisation over Administration by Notables. In G. Roth. \& C. Wittich. (Eds.). Economy and Society: An Outline of Interpretive Sociology (pp. 973-975). (H. H. Gerth \& C. Wright Mills, Trans.). Berkeley, CA: University of California Press.

Weber, R. P. (1990). Basis Content Analysis (2nd ed.). Thousand Oaks, CA: Sage Publications.

Wheeldon, J., \& Ahlberg, M. (2012). Visualising Social Science Research: Maps, methods and meaning. California: Sage. 
Wicks, A., Gilbert, D., \& Freeman, R. E. (1994). A Feminist Reinterpretation of the Stakeholder Concept. Business Ethics Quarterly, 4(4), 475-497.

Wickert, C. (2016). "Political" Corporate Social Responsibility in Small and Medium-Sized Enterprises: A Conceptual Framework. Business and Society, 55(6), 792-824.

Wickert, C., Scherer, A. G., \& Spence, L. J. (2016). Walking and Talking Corporate Social Responsibility: Implications of Firm Size and Organizational Cost. Journal of Management Studies, 53(7), 1169-1196.

Wildermuth, C., De Mello e Souza, C. A., \& Kozitza, T. (2017). Circles of Ethics: The Impact of Proximity on Moral Reasoning. Journal of Business Ethics, 40(1), 126.

Wilson, E. (1980). Social responsibility of business: What are the small business perspectives? Journal of Small Business Management, 18, 17-24.

Windsor, D. (2001). The Future of Corporate Social Responsibility. The International Journal of Organizational Analysis, 9(3), 225-256.

Windsor, D. (2006). Corporate Social Responsibility: Three Key Approaches. Journal of Management Studies, 43(1), 93-114.

Wood, D. J. (1991). Corporate Social Performance Revisited. Academy of Management Review, 16(4), 691-718.

Wood, D. J., \& Jones, R. E. (1995). Stakeholder mismatching: A theoretical problem in empirical research on corporate social performance. The International Journal of Organizational Analysis, 3(3), 229-267.

Zouboulakis, M. S. (2001). From Mill to Weber: the meaning of the concept of economic rationality. The European Journal of the History of Economic Thought, 
8(1), 30-41. 


\section{Appendices}

\section{Appendix 3.1: Literature Overview: CSR in SMEs and Relevant Disciplines}

\begin{tabular}{|c|c|c|}
\hline \multicolumn{3}{|c|}{ Literature Overview: Business Ethics and CSR in SMEs } \\
\hline Topic & $\begin{array}{l}\text { Disciplinary } \\
\text { Perspectives }\end{array}$ & Authors (chronological order) \\
\hline $\begin{array}{l}\text { Decision-making, } \\
\text { motivations and } \\
\text { influence of } \\
\text { entrepreneur/owner- } \\
\text { manager }\end{array}$ & $\begin{array}{l}\text { Business ethics, } \\
\text { entrepreneurship, small } \\
\text { business. }\end{array}$ & $\begin{array}{l}\text { Baden, Harwood \& Woodward (2011); Burton \& Goldsby } \\
\text { (2009); Campopiano, De Massis \& Cassia (2012); D’Aprile \& } \\
\text { Mannarini (2012); Fassin, Van Rossem \& Buelens (2011); } \\
\text { Garay \& Font (2012); Humphreys, Robin, Reidenbach \& } \\
\text { Moak (1993); McVea (2009); Payne \& Joyner (2006); } \\
\text { Rutherford, Buller \& Stebbins (2009) }\end{array}$ \\
\hline $\begin{array}{l}\text { The moral nature of } \\
\text { entrepreneurship } \\
\text { and entrepreneurs }\end{array}$ & $\begin{array}{l}\text { Business/applied ethics; } \\
\text { development, general } \\
\text { management, geography, } \\
\text { entrepreneurship, } \\
\text { organisation studies, } \\
\text { political economy }\end{array}$ & $\begin{array}{l}\text { Anderson \& Smith (2007); Baumol (1990); Blackburn \& } \\
\text { McGhee (2007); Bucar, Glas \& Hisrich (2003); Bucar \& } \\
\text { Hisrich (2001); Buchholz \& Rosenthal (2005); Brenkert } \\
\text { (2009); Dees \& Starr (1992); Dunham (2010); Jones \& Spicer } \\
\text { (2005); Webb, Tihanyi, Ireland \& Sirmon (2009) }\end{array}$ \\
\hline $\begin{array}{l}\text { Language and } \\
\text { discourse }\end{array}$ & Business ethics & $\begin{array}{l}\text { Baden \& Harwood (2013); Fassin, Van Rossem \& Buelens } \\
\text { (2013); Lähdesmäki (2005); Lähdesmäki (2012); Murillo \& } \\
\text { Lozano (2006); Spence \& Lozano (2000) }\end{array}$ \\
\hline Influence of religion & Business ethics & Graafland, Mazereeuw \& Yahia (2006) \\
\hline Philanthropy & $\begin{array}{l}\text { Business ethics, CSR, } \\
\text { development, small } \\
\text { business, strategy }\end{array}$ & $\begin{array}{l}\text { Amato \& Amato (2007); Baumann-Pauly, Wickert, Spence \& } \\
\text { Scherer (2013); Brammer \& Millington (2004); Lähdesmäki \& } \\
\text { Takala (2012); Schaper \& Savery (2004); Thompson, Smith \& } \\
\text { Hood (1993) }\end{array}$ \\
\hline $\begin{array}{l}\text { Nature of CSR and } \\
\text { ethics in SMEs/size } \\
\text { implications }\end{array}$ & $\begin{array}{l}\text { Business ethics, small } \\
\text { business, general } \\
\text { management, CSR, } \\
\text { strategy }\end{array}$ & $\begin{array}{l}\text { Jenkins (2006); Lepoutre \& Heene (2006); Inyang, (2013); } \\
\text { Spence, (1999); Wilson, (1980) }\end{array}$ \\
\hline $\begin{array}{l}\text { Theory } \\
\text { Development }\end{array}$ & $\begin{array}{l}\text { Business ethics, general } \\
\text { management, small } \\
\text { business }\end{array}$ & $\begin{array}{l}\text { Blundel, Spence \& Zerbinati (2010); Jenkins (2004); Spence } \\
\text { (2004); Spence (2014); Spence (2016); Spence \& Rutherfoord } \\
\text { (2001); Udayasankar (2008); Wickert (2016) }\end{array}$ \\
\hline $\begin{array}{l}\text { Focused empirical } \\
\text { evidence on the } \\
\text { nature of ethics and } \\
\text { social responsibility } \\
\text { in small firms }\end{array}$ & $\begin{array}{l}\text { Business ethics, corporate } \\
\text { governance; CSR; } \\
\text { entrepreneurship, } \\
\text { environmental } \\
\text { management, innovation, } \\
\text { public relations, regional } \\
\text { general business; small } \\
\text { business }\end{array}$ & $\begin{array}{l}\text { USA - Hornsby, Kuratko, Naffziger, LaFollette \& Hodgetts } \\
\text { (1994) } \\
\text { UK - Quinn (1997) } \\
\text { Italy - Longo, Mura \& Bonoli (2005) } \\
\text { Ireland - Sweeney (2007) } \\
39 \text { countries - De Clercq, Danis \& Dakhli (2010) } \\
\text { Denmark - Nielsen \& Thomsen (2019) } \\
\text { Germany - Hammann, Habisch \& Pechlaner } \\
\text { (2009) } \\
\text { Norway - Von Weltzien Høivik \& Melé } \\
\text { (2009) } \\
\text { Sweden - Blombäck \& Wigren (2009) } \\
\text { China - Liu \& Fong (2010) } \\
\text { Bangladesh - Rahim \& Wisuttisak (2012) } \\
\text { India - Gupta \& Kalra (2012) } \\
\text { Malaysia - Nejati \& Amran (2012) } \\
\text { Singapore - Lee, Mak \& Pang (2012) } \\
\text { Cameroon - Demuijnck \& Ngnodjom (2013) } \\
\text { Europe - Koos (2012); Spence \& Perrini (2009) } \\
\text { Micro firms - Campin, Barraket \& Luke (2013); Courrent \& } \\
\text { Gundolf (2009) } \\
\text { Medium firms - Preuss \& Perschke (2010) }\end{array}$ \\
\hline Strategy & $\begin{array}{l}\text { Business ethics, } \\
\text { communication, CSR, } \\
\text { entrepreneurship, } \\
\text { technology management }\end{array}$ & $\begin{array}{l}\text { General - Castka, Balzarova, Bamber, \& Sharp (2004); } \\
\text { Fenwick (2010); Gelbmann, (2010); Graafland, Van de Ven \& } \\
\text { Stoffele (2003); Russo \& Tencati (2009); Sarbutts (2003); } \\
\text { Schlierer et al. (2012) }\end{array}$ \\
\hline
\end{tabular}




\begin{tabular}{|c|c|c|}
\hline & & $\begin{array}{l}\text { Innovation - Anokhin \& Schulze (2009); Biondi, Iraldo \& } \\
\text { Meredith (2002) } \\
\text { Performance - Avram \& Kühne (2008); Tantalo, Caroli, \& } \\
\text { Vanevenhoven (2012); Torugsa, O’Donohue, \& Hecker } \\
\text { (2013) }\end{array}$ \\
\hline Employees & $\begin{array}{l}\text { Business ethics, corporate } \\
\text { governance, employee } \\
\text { relations, health, } \\
\text { sociology }\end{array}$ & $\begin{array}{l}\text { Christopher (2003); Davies \& Crane (2010); Donia, Tetrault } \\
\text { Sirsly \& Ronen (2017); Eakin \& MacEachen (1998); Granerud } \\
\text { (2011); Jones, Marshall \& Mitchell (2007); Massey (2004) }\end{array}$ \\
\hline Community & $\begin{array}{l}\text { Business ethics, CSR, } \\
\text { entrepreneurship, socio- } \\
\text { economics, voluntary } \\
\text { sector }\end{array}$ & $\begin{array}{l}\text { Besser \& Miller (2004); Besser (2012); Besser, Miller \& } \\
\text { Perkins (2006); Campin, Barraket, \& Luke (2013); Curran, } \\
\text { Rutherfoord \& Smith (2000); Fisher, Geenan, Jurcevic, } \\
\text { McClintock \& Davis (2009); Koos (2012); Lähdesmäki \& } \\
\text { Suutari (2012); Litz \& Samu (2008) }\end{array}$ \\
\hline $\begin{array}{l}\text { Stakeholders, supply } \\
\text { chains, networks } \\
\text { and social capital }\end{array}$ & $\begin{array}{l}\text { Business ethics, corporate } \\
\text { governance, small } \\
\text { business, supply chain } \\
\text { management }\end{array}$ & $\begin{array}{l}\text { Baden, Harwood \& Woodward (2011); Ciliberti, Baden \& } \\
\text { Harwood, (2009); Rawlings (2012); Del Baldo (2012); Dyer \& } \\
\text { Handler, (1994); Fuller \& Tian (2006); Lähdesmäki, Siltaoja } \\
\text { \& Spence (2017); Lund-Thomsen \& Pillay (2012); Mitchell, } \\
\text { Agle, Chrisman \& Spence (2011), Murillo \& Lozano (2009); } \\
\text { Perrini (2006); Russo \& Perrini (2010); Sen \& Cowley (2013); } \\
\text { Spence \& Schmidpeter (2003); Spence, Schmidpeter \& } \\
\text { Habisch (2003); Von Weltzien Høivik \& Shankar (2011) }\end{array}$ \\
\hline $\begin{array}{l}\text { Development and } \\
\text { developing countries }\end{array}$ & $\begin{array}{l}\text { Business ethics, CSR, } \\
\text { economics, strategy }\end{array}$ & $\begin{array}{l}\text { Ahmad \& Ramayah (2012) Hamann, Agbazue, Kapelus \& } \\
\text { Hein (2005); Jamali, Zanhour \& Keshishian (2009); } \\
\text { Luetkenhorst (2004); Luken \& Stares (2005); Vives (2006) }\end{array}$ \\
\hline $\begin{array}{l}\text { CSR formalisation, } \\
\text { reporting and } \\
\text { communication }\end{array}$ & CSR, marketing, strategy & $\begin{array}{l}\text { Fassin (2008); Fraj-Andrés, López-Pérez, Melero-Polo \& } \\
\text { Vázquez-Carrasco (2012); Gallo \& Christensen (2011) }\end{array}$ \\
\hline $\begin{array}{l}\text { Sustainability and } \\
\text { environmental ethics }\end{array}$ & $\begin{array}{l}\text { Sustainability, } \\
\text { environmental ethics, } \\
\text { strategy, business ethics, } \\
\text { CSR. }\end{array}$ & $\begin{array}{l}\text { Cassells \& Lewis (2011); Revell, Stokes \& Chen (2009); } \\
\text { Tilley (2000); Uhlaner, Berent-Braun, Jeurissen \& de Wit } \\
\text { (2012) }\end{array}$ \\
\hline $\begin{array}{l}\text { CSR in SME } \\
\text { literature reviews, } \\
\text { research agenda and } \\
\text { editorials }\end{array}$ & $\begin{array}{l}\text { CSR, business ethics, } \\
\text { SME }\end{array}$ & $\begin{array}{l}\text { Moore \& Spence (2006); Morsing \& Perrini (2009); Spence } \\
\text { (2007); Spence \& Rutherfoord (2003); Vázquez-Carrasco \& } \\
\text { Lopez-Perez (2013) }\end{array}$ \\
\hline
\end{tabular}

\section{Appendix 4.1: FSB Regional Meetings and Focus Group Locations}

\begin{tabular}{|l|l|l|}
\hline FSB Region & Location & Date \\
\hline North Wales Region & Location 1 & $3^{\text {rd }}$ May 2016 \\
\hline Mid Wales Region & Location 2 & $27^{\text {th }}$ May 2016 \\
\hline South Wales Region & Location 3 & $28^{\text {th }}$ July 2016 \\
\hline South Wales Region & Location 4 & $28^{\text {th }}$ July 2016 \\
\hline
\end{tabular}




\section{Appendix 4.2: Number of Focus Group Attendees}

\begin{tabular}{|l|l|l|l|}
\hline Focus group location & Number of facilitators & $\begin{array}{c}\text { Number of } \\
\text { participants }\end{array}$ & $\begin{array}{c}\text { Total } \\
\text { number of } \\
\text { attendees }\end{array}$ \\
\hline Location 1 & 3 & 9 & 12 \\
\hline Location 2 & 1 & 7 & 8 \\
\hline Location 3 & 2 & 5 & 7 \\
\hline Location 4 & 2 & 6 & 8 \\
\hline Total & 8 & 27 & 35 \\
\hline Total individuals & 4 & 27 & 31 \\
\hline
\end{tabular}

\section{Appendix 4.3: Focus Group SME Profiles}

\begin{tabular}{|c|c|c|c|c|}
\hline \multicolumn{5}{|c|}{ Focus Group - Location 1} \\
\hline Participant & $\begin{array}{l}\text { Number of } \\
\text { employees }\end{array}$ & SME size & Sector & $\begin{array}{l}\text { Position of the } \\
\text { participant }\end{array}$ \\
\hline $\mathrm{P} 1$ & 3 & Micro & $\begin{array}{l}\text { Information and } \\
\text { communication } \\
\text { technology }\end{array}$ & $\begin{array}{l}\text { Owner- } \\
\text { manager }\end{array}$ \\
\hline $\mathrm{P} 2$ & 1 & Micro & $\begin{array}{l}\text { Arts, entertainment } \\
\text { and recreation }\end{array}$ & $\begin{array}{l}\text { Owner- } \\
\text { manager }\end{array}$ \\
\hline P3 & 2 & Micro & $\begin{array}{l}\text { Accommodation } \\
\text { and food service } \\
\text { activities (tourism) }\end{array}$ & $\begin{array}{l}\text { Owner- } \\
\text { manager }\end{array}$ \\
\hline $\mathrm{P} 4$ & 65 & Medium & $\begin{array}{l}\text { Accommodation } \\
\text { and food service } \\
\text { activities (tourism) }\end{array}$ & $\begin{array}{l}\text { Business } \\
\text { development } \\
\text { manager (key } \\
\text { decision } \\
\text { maker) }\end{array}$ \\
\hline P5 & 8 & Micro & Social enterprise & $\begin{array}{l}\text { Owner- } \\
\text { manager }\end{array}$ \\
\hline P6 & 2 & Micro & $\begin{array}{l}\text { Accommodation } \\
\text { and food service } \\
\text { activities (tourism) }\end{array}$ & $\begin{array}{l}\text { Owner- } \\
\text { manager }\end{array}$ \\
\hline P7 & 5 & Micro & $\begin{array}{l}\text { Professional, } \\
\text { scientific and } \\
\text { technical activities }\end{array}$ & $\begin{array}{l}\text { Owner- } \\
\text { manager }\end{array}$ \\
\hline P8 & 5 & Micro & $\begin{array}{l}\text { Accommodation } \\
\text { and food service } \\
\text { activities (tourism) }\end{array}$ & $\begin{array}{l}\text { Owner- } \\
\text { manager }\end{array}$ \\
\hline
\end{tabular}




\begin{tabular}{|l|l|l|l|l|}
\hline P9 & 7 & Micro & $\begin{array}{l}\text { Accommodation } \\
\text { and food service } \\
\text { activities (tourism) }\end{array}$ & $\begin{array}{l}\text { Owner- } \\
\text { manager }\end{array}$ \\
\hline
\end{tabular}

\begin{tabular}{|c|c|c|c|c|}
\hline \multicolumn{5}{|c|}{ Focus Group - Location 2} \\
\hline Participant & $\begin{array}{l}\text { Number of } \\
\text { employees }\end{array}$ & SME size & Sector & $\begin{array}{l}\text { Position of } \\
\text { participant }\end{array}$ \\
\hline P10 & 3 & Micro & Social enterprise & Owner-manager \\
\hline P11 & 1 & Micro & $\begin{array}{l}\text { Information and } \\
\text { communication } \\
\text { technology }\end{array}$ & Owner-manager \\
\hline P12 & 53 & Medium & Education & $\begin{array}{l}\text { Key decision } \\
\text { maker }\end{array}$ \\
\hline P13 & 9 & Micro & $\begin{array}{l}\text { Wholesale and } \\
\text { retail trade }\end{array}$ & Owner-manager \\
\hline P14 & 19 & Small & Construction & Owner-manager \\
\hline P15 & 230 & Medium & $\begin{array}{l}\text { Professional, } \\
\text { scientific and } \\
\text { technical activities }\end{array}$ & $\begin{array}{l}\text { Key decision } \\
\text { maker }\end{array}$ \\
\hline P16 & 2 & Micro & $\begin{array}{l}\text { Arts, } \\
\text { entertainment and } \\
\text { recreation }\end{array}$ & Owner-manager \\
\hline
\end{tabular}

\begin{tabular}{|c|c|c|c|c|}
\hline \multicolumn{5}{|c|}{ Focus Group - Location 3} \\
\hline Participant & $\begin{array}{l}\text { Number of } \\
\text { employees }\end{array}$ & SME size & Sector & $\begin{array}{l}\text { Position of } \\
\text { participant }\end{array}$ \\
\hline P17 & 1 & Micro & $\begin{array}{l}\text { Energy and } \\
\text { environment }\end{array}$ & Owner-manager \\
\hline P18 & 1 & Micro & $\begin{array}{l}\text { Professional, } \\
\text { scientific and } \\
\text { technical activities }\end{array}$ & Owner-maanger \\
\hline P19 & 1 & Micro & Education & Owner-manager \\
\hline $\mathrm{P} 20$ & 4 & Micro & $\begin{array}{l}\text { Wholesale and } \\
\text { retail trade }\end{array}$ & Owner-manager \\
\hline $\mathrm{P} 21$ & 7 & Micro & Engineering & Owner-manager \\
\hline
\end{tabular}

Focus Group - Location 4

\begin{tabular}{|l|l|l|l|l|}
\hline Participant & $\begin{array}{l}\text { Number of } \\
\text { employees }\end{array}$ & SME size & Sector & $\begin{array}{l}\text { Position of } \\
\text { participant }\end{array}$ \\
\hline
\end{tabular}




\begin{tabular}{|l|l|l|l|l|}
\hline P22 & 10 & Small & $\begin{array}{l}\text { Wholesale and } \\
\text { retail trade }\end{array}$ & $\begin{array}{l}\text { Key decision } \\
\text { maker }\end{array}$ \\
\hline P23 & 3 & Micro & $\begin{array}{l}\text { Wholesale and } \\
\text { retail trade }\end{array}$ & Owner-manager \\
\hline P24 & 1 & Micro & $\begin{array}{l}\text { Financial and } \\
\text { insurance } \\
\text { activities }\end{array}$ & Owner-manager \\
\hline P25 & 1 & Micro & $\begin{array}{l}\text { Professional, } \\
\text { scientific and } \\
\text { technical activities }\end{array}$ & Owner-manager \\
\hline P26 & 1 & Micro & $\begin{array}{l}\text { Other service } \\
\text { activities }\end{array}$ & Owner-manager \\
\hline P27 & 1 & Micro & Education & Owner-manager \\
\hline
\end{tabular}

Appendix 4.4: Percentage of Focus Group SME Sizes

\begin{tabular}{|l|l|}
\hline Size & Percentage \\
\hline Micro $(<10)$ & $81.4 \%$ \\
\hline Small $(<50)$ & $7.4 \%$ \\
\hline Medium-sized $(<250)$ & $11.1 \%$ \\
\hline
\end{tabular}


Appendix 4.5: Focus Group Participant Booklet

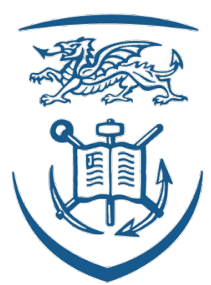

Swansea University

Prifysgol Abertawe

\section{Corporate Social}

Responsibility in Small and

Medium-Sized Enterprises

in Wales

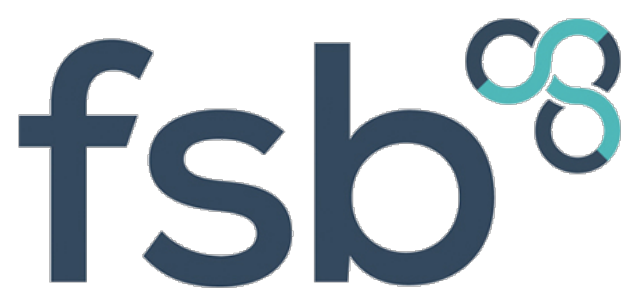

Experts in Business 
This project is a joint collaboration between The Federation of Small Businesses and Swansea University Management School and is designed to help us understand and promote the important contribution small businesses make to Wales.

The economic contribution is crucial and can be at least partially quantified. What is less obvious is the work that many, if not most, small businesses do in their communities and beyond. This is sometimes called corporate social responsibility, mainly among larger firms, or described as responsible business practice or even good citizenship.

However, there is no doubt that even where many of us give such practices no name at all, most small businesses engage in these kinds of good behaviours. It is time that this was fully reflected in how small businesses are viewed by stakeholders from community to government.

In 2010 the FSB, along with Business in the Community, took part in a wide ranging study into these matters commissioned by the Welsh Government through a task and finish group. There was plentiful evidence that small business made a significant contribution to Wales that was not always easy to see.

Now in 2016 the FSB and Swansea University with their new Centre for Responsible Organisation and Social Innovation are working together again to conduct an updated study to inform policy and practice into the next political cycle and beyond.

Therefore, this focus group has been arranged in order to speak to FSB members such as you, to seek views and information that will inform this study. There will be three focus groups, one in each of south, mid and north Wales, in order to understand the basic issues. Then a more in-depth data gathering exercise will build on this to give us a detailed picture of the situation.

We hope you will join us in supporting this exciting initiative. It is one that will help the FSB in their lobbying and policy advice, as well as making clear the vital, broad contribution made by small businesses to the economic and social landscape of Wales as a whole.

With regards to this I would like to ask for your consent to record this focus group and for you to fill out the consent form. All material containing personal details will be anonymised. Any information collated during the process of the research will be treated in accordance with the Data Protection Act. Access to identifiable research materials will be restricted to the researcher and under no circumstances (except that decreed by law), will the material be available to any other party. If you have any questions regarding the study then don't hesitate to ask.

For more information contact-635801@swansea.ac.uk 
Participant Information

\begin{tabular}{|l|l|l|}
\hline Main Company Activity & No of Employees & $\begin{array}{l}\text { Position of Respondent }(\mathrm{O} / \mathrm{M}= \\
\text { owner/manager D = director) }\end{array}$ \\
\hline & & \\
& & \\
\hline
\end{tabular}




\section{Participant Consent Form}

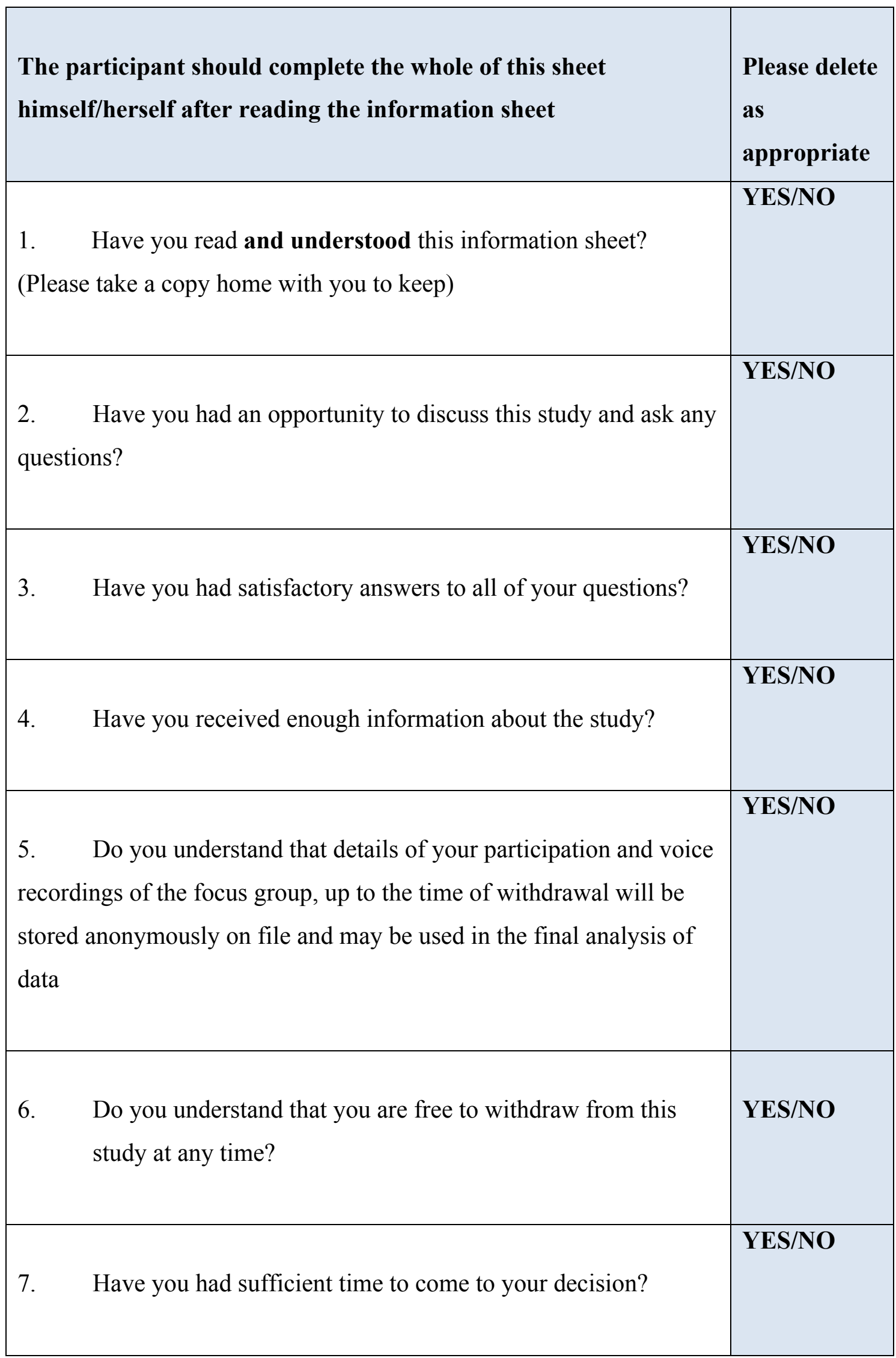




\begin{tabular}{|l|l|}
\hline 8. Do you agree to participate in this study? & YES/NO \\
\hline
\end{tabular}

\section{PARTICIPANT}

Signed

Date

Name

(BLOCKLETTERS)

I have explained the study to the above participant and they have indicated their willingness to take part.

\section{INVESTIGATOR}

Signed

Date

Name

(BLOCKLETTERS) 


\section{Task 1}

Who are your main stakeholders? List up to 10 stakeholders in order of how important you think they are to your business. A stakeholder can be any group or individual that interacts with your business.

1.

2.

3.

4.

5.

6.

7.

8.

9.

10. 


\section{Task 2}

Some recent exciting research into small business social responsibility has split the priorities of small businesses into four different areas.

We would like you to rank these in order of how important you feel they are for YOUR business (as opposed to businesses in general). There is no 'right and wrong' here - please simply order them according to the way you feel for your business.

The four components (with a brief explanation) are:

\section{Philanthropic responsibility}

(An example might be the priority given to charitable donations or giving time, to promote the welfare of others outside the organisation)

\section{Personal Integrity}

(The personal reputation and integrity of the owner manager - how important is this as a priority?)

\section{Care for others}

(This means the priority given to meeting the needs of others for whom the business has a responsibility.

\section{Survival}

(This means the firm being economically viable and sufficiently in line with legal requirements to ensure continued operation.)

Please rank below:

1)

2)

3)

4) 
Appendix 4.6: Focus Group: Topics of Discussion and Supporting Literature

\begin{tabular}{|l|l|l|}
\hline Stakeholders & General Topics & Source \\
\hline & Prioritisation & $\begin{array}{l}\text { Freeman (1970) } \\
\text { Sen \& Cowley (2013) } \\
\text { Spence (2016) }\end{array}$ \\
\hline CSR & Relationships & $\begin{array}{l}\text { Spence (2014) } \\
\text { Spence (2016) } \\
\text { Murillo \& Lozano (2009) } \\
\text { Russo \& Perrini (2010) }\end{array}$ \\
\hline & Conceptualisation & $\begin{array}{l}\text { Jenkins (2004) } \\
\text { Spence (2014) }\end{array}$ \\
\hline & Engagement & $\begin{array}{l}\text { Jenkins (2004) } \\
\text { Spence (2016) }\end{array}$ \\
\hline & Language & Baden \& Harwood (2013) \\
\hline & Drivers & $\begin{array}{l}\text { Spence \& Rutherfoord (2001) } \\
\text { Murillo \& Lozano (2006) } \\
\text { Jamali et al. (2009) } \\
\text { Sen \& Cowley (2013) }\end{array}$ \\
& & $\begin{array}{l}\text { Jenkins (2006) } \\
\text { Wickert et al. (2016) }\end{array}$ \\
\hline & Barriers & $\begin{array}{l}\text { Murillo \& Lozano (2006) } \\
\text { Fassin (2008) } \\
\text { Jamali et al. (2009) } \\
\text { Baumann-Pauly et al. (2013) }\end{array}$ \\
\hline & Budget/measurement \\
& &
\end{tabular}




\section{Appendix 4.7: Initial Approach Email}

Dear...,

I hope that you don't mind me contacting you, my name is Fern Davies and I'm a third year PhD student from the School of Management at Swansea University.

I'm currently conducting a study into the way that small and medium-sized businesses prioritise responsibilities. I am at the data collection stage of interviewing owner-managers of SMEs and would like to invite you to participate.

The interview would last for approximately an hour and once the project begins, your participation remains entirely voluntary. This means that you can withdraw at any time and ask that your contribution is removed. In addition, all interviews will remain confidential since the eventual reporting of the study will be made anonymous.

I would very much welcome your contribution to this study, my email is 635801@swansea.ac.uk if you require more information.

Kind regards,

Fern Davies 


\section{Appendix 4.8: Interview Participant and SME Profiles}

\begin{tabular}{|c|c|c|c|c|c|c|c|c|}
\hline \multicolumn{9}{|c|}{ Interview Participant Profiles } \\
\hline Participant & Gender & $\begin{array}{l}\text { Position of } \\
\text { the } \\
\text { participant }\end{array}$ & $\begin{array}{l}\text { No. } \\
\text { employees }\end{array}$ & $\begin{array}{l}\text { Type of } \\
\text { SME }\end{array}$ & $\begin{array}{l}\text { Business } \\
\text { activity }\end{array}$ & Sector & $\begin{array}{l}\text { Years } \\
\text { SME in } \\
\text { business }\end{array}$ & $\begin{array}{l}\text { Online } \\
\text { or } \\
\text { virtual }\end{array}$ \\
\hline P1 & Female & $\begin{array}{l}\text { Owner- } \\
\text { manager }\end{array}$ & 14 & Small & $\begin{array}{l}\text { Creative } \\
\text { agency - } \\
\text { marketing } \\
\text { strategy }\end{array}$ & $\begin{array}{l}\text { Information } \\
\text { and } \\
\text { communicatio } \\
\text { n technology }\end{array}$ & 12 years & Yes \\
\hline P2 & Male & $\begin{array}{l}\text { Owner- } \\
\text { manager }\end{array}$ & 34 & Small & Recruitment & $\begin{array}{l}\text { Professional, } \\
\text { scientific and } \\
\text { technical } \\
\text { activities }\end{array}$ & $\begin{array}{l}13 \text { years } \\
\text { (since } \\
2004)\end{array}$ & No \\
\hline P3 & Male & $\begin{array}{l}\text { Owner- } \\
\text { manager }\end{array}$ & 5 & Micro & Recruitment & $\begin{array}{l}\text { Professional, } \\
\text { scientific and } \\
\text { technical } \\
\text { activities }\end{array}$ & 4 years & Yes \\
\hline $\mathrm{P} 4$ & Male & $\begin{array}{l}\text { Owner- } \\
\text { manager }\end{array}$ & 5 & Micro & $\begin{array}{l}\text { Web } \\
\text { development } \\
\text { and } \\
\text { consultancy }\end{array}$ & $\begin{array}{l}\text { Information } \\
\text { and } \\
\text { communicatio } \\
\text { n technology }\end{array}$ & 13 years & Yes \\
\hline P5 & Male & $\begin{array}{l}\text { Owner- } \\
\text { manager }\end{array}$ & 3 & Micro & $\begin{array}{l}\text { Tourism - } \\
\text { extreme } \\
\text { sports and } \\
\text { retail }\end{array}$ & $\begin{array}{l}\text { Arts, } \\
\text { entertainment } \\
\text { and recreation }\end{array}$ & 13 years & Yes \\
\hline P6 & Male & $\begin{array}{l}\text { Manager } \\
\text { (Key } \\
\text { decision } \\
\text { maker) }\end{array}$ & 13 & Small & $\begin{array}{l}\text { Social } \\
\text { enterprise } \\
\text { (coffee } \\
\text { house) }\end{array}$ & $\begin{array}{l}\text { Social } \\
\text { enterprise }\end{array}$ & 1.5 years & No \\
\hline P7 & Female & $\begin{array}{l}\text { Owner- } \\
\text { manager }\end{array}$ & 0 & Micro & $\begin{array}{l}\text { Fashion - } \\
\text { clothing/retail }\end{array}$ & $\begin{array}{l}\text { Wholesale } \\
\text { and retail } \\
\text { trade }\end{array}$ & 3 years & Yes \\
\hline P8 & Male & $\begin{array}{l}\text { Owner- } \\
\text { manager }\end{array}$ & 8 & Micro & $\begin{array}{l}\text { Licensed } \\
\text { wine bar }\end{array}$ & $\begin{array}{l}\text { Arts, } \\
\text { entertainment } \\
\text { and recreation }\end{array}$ & 7 years & No \\
\hline P9 & Female & $\begin{array}{l}\text { Owner- } \\
\text { manager }\end{array}$ & 15 & Small & $\begin{array}{l}\text { Chain of } \\
\text { Cafes }\end{array}$ & $\begin{array}{l}\text { Accommodati } \\
\text { on and food } \\
\text { service } \\
\text { activities }\end{array}$ & 2 years & No \\
\hline P10 & Male & $\begin{array}{l}\text { Owner- } \\
\text { manager }\end{array}$ & 105 & Medium & $\begin{array}{l}\text { Furniture } \\
\text { shops }\end{array}$ & $\begin{array}{l}\text { Wholesale } \\
\text { and retail } \\
\text { trade }\end{array}$ & $\begin{array}{l}125 \text { years } \\
\text { (37 years } \\
\text { current } \\
\text { owner- } \\
\text { manager) }\end{array}$ & $\begin{array}{l}\text { Yes } \\
\text { both }\end{array}$ \\
\hline P11 & Female & Owner- & 1 & Micro & Beauty & Other service & 3 years & No \\
\hline
\end{tabular}




\begin{tabular}{|c|c|c|c|c|c|c|c|c|}
\hline & & manager & & & & activities & & \\
\hline P12 & Male & $\begin{array}{l}\text { Owner- } \\
\text { manager }\end{array}$ & 3 & Micro & $\begin{array}{l}\text { Painter and } \\
\text { decorator }\end{array}$ & Construction & 25 years & No \\
\hline P13 & Male & $\begin{array}{l}\text { Owner- } \\
\text { manager }\end{array}$ & 108 & Medium & $\begin{array}{l}\text { Pie and pasty } \\
\text { factory }\end{array}$ & $\begin{array}{l}\text { Accommodati } \\
\text { on and food } \\
\text { service } \\
\text { activities }\end{array}$ & $\begin{array}{l}81 \text { years } \\
3 \text { years }\end{array}$ & \\
\hline P14 & Female & $\begin{array}{l}\text { Owner- } \\
\text { manager }\end{array}$ & 2 & Micro & $\begin{array}{l}\text { Baby and } \\
\text { toddler } \\
\text { swimming } \\
\text { school }\end{array}$ & $\begin{array}{l}\text { Arts, } \\
\text { entertainment } \\
\text { and recreation }\end{array}$ & 1 year & No \\
\hline P15 & Female & $\begin{array}{l}\text { Owner- } \\
\text { manager }\end{array}$ & 0 & Micro & $\begin{array}{l}\text { Arts and } \\
\text { crafts }\end{array}$ & $\begin{array}{l}\text { Arts, } \\
\text { entertainment } \\
\text { and recreation }\end{array}$ & 4 years & Yes \\
\hline P16 & Female & $\begin{array}{l}\text { Owner- } \\
\text { manager }\end{array}$ & 0 & Micro & $\begin{array}{l}\text { Bespoke } \\
\text { bridal wear } \\
\text { designer }\end{array}$ & $\begin{array}{l}\text { Arts, } \\
\text { entertainment } \\
\text { and recreation }\end{array}$ & 12 years & Yes \\
\hline P17 & Female & $\begin{array}{l}\text { Owner- } \\
\text { manager }\end{array}$ & 3 & Micro & $\begin{array}{l}\text { Underwear } \\
\text { retail shop }\end{array}$ & $\begin{array}{l}\text { Wholesale } \\
\text { and retail } \\
\text { trade }\end{array}$ & 10 years & Yes \\
\hline P18 & Male & $\begin{array}{l}\text { Owner- } \\
\text { manager }\end{array}$ & 4 & Micro & $\begin{array}{l}\text { Chemical } \\
\text { metallurgy }\end{array}$ & Engineering & 16 years & No \\
\hline P19 & Male & $\begin{array}{l}\text { Owner- } \\
\text { manager }\end{array}$ & 18 & Small & Photography & $\begin{array}{l}\text { Arts, } \\
\text { entertainment } \\
\text { and recreation }\end{array}$ & 5 years & Yes/No \\
\hline P20 & Female & $\begin{array}{l}\text { Owner- } \\
\text { manager }\end{array}$ & 2 & Micro & $\begin{array}{l}\text { Marketing } \\
\text { consultancy }\end{array}$ & $\begin{array}{l}\text { Professional, } \\
\text { scientific and } \\
\text { technical } \\
\text { activities }\end{array}$ & 1 year & $\mathrm{Yes} / \mathrm{No}$ \\
\hline P21 & Female & $\begin{array}{l}\text { Owner- } \\
\text { manager }\end{array}$ & 2 & Micro & $\begin{array}{l}\text { Card and gift } \\
\text { production }\end{array}$ & $\begin{array}{l}\text { Arts, } \\
\text { entertainment } \\
\text { and recreation }\end{array}$ & 20 years & Yes/No \\
\hline P22 & Female & $\begin{array}{l}\text { Owner- } \\
\text { manager }\end{array}$ & 4 & Micro & $\begin{array}{l}\text { Creative } \\
\text { digital agency }\end{array}$ & $\begin{array}{l}\text { Information } \\
\text { and } \\
\text { communicatio } \\
\text { n technology }\end{array}$ & 16 years & Yes \\
\hline P23 & Female & $\begin{array}{l}\text { Owner- } \\
\text { manager }\end{array}$ & 1 & Micro & $\begin{array}{l}\text { Marketing } \\
\text { collateral }\end{array}$ & $\begin{array}{l}\text { Wholesale } \\
\text { and retail } \\
\text { trade }\end{array}$ & 5 years & $\begin{array}{l}\text { Yes/No } \\
?\end{array}$ \\
\hline P24 & Female & $\begin{array}{l}\text { Owner- } \\
\text { manager }\end{array}$ & 6 & Micro & Day nursery & Education & 4 years & No \\
\hline $\mathrm{P} 25$ & Male & $\begin{array}{l}\text { Owner- } \\
\text { manager }\end{array}$ & 54 & Medium & $\begin{array}{l}\text { Medium- } \\
\text { heavy } \\
\text { engineering } \\
\text { (fabricating/ } \\
\text { machinery) }\end{array}$ & Engineering & 48 years & No \\
\hline P26 & Female & $\begin{array}{l}\text { Owner- } \\
\text { manager }\end{array}$ & 25 & Small & Hairdressers & $\begin{array}{l}\text { Arts, } \\
\text { entertainment } \\
\text { and recreation }\end{array}$ & 21 years & No \\
\hline
\end{tabular}




\begin{tabular}{|l|l|l|l|l|l|l|l|l|}
\hline P27 & Male & $\begin{array}{l}\text { Owner- } \\
\text { manager }\end{array}$ & 17 & Small & $\begin{array}{l}\text { Government } \\
\text { funded } \\
\text { apprenticeshi } \\
\text { p provider }\end{array}$ & Education & 5 years & No \\
\hline P28 & Female & $\begin{array}{l}\text { Owner- } \\
\text { manager }\end{array}$ & 2 & Micro & Fashion shop & $\begin{array}{l}\text { Wholesale } \\
\text { and retail } \\
\text { trade }\end{array}$ & 13 years & Yes \\
\hline P29 & Female & $\begin{array}{l}\text { Owner- } \\
\text { manager }\end{array}$ & 3 & Micro & Accountants & $\begin{array}{l}\text { Financial and } \\
\text { insurance } \\
\text { activities }\end{array}$ & 17 years & No \\
\hline P30 & Female & $\begin{array}{l}\text { Owner- } \\
\text { manager }\end{array}$ & 12 & Small & $\begin{array}{l}\text { Petrol station, } \\
\text { workshop and } \\
\text { police vehicle } \\
\text { recovery }\end{array}$ & $\begin{array}{l}\text { Other service } \\
\text { activities }\end{array}$ & 29 years & No \\
\hline
\end{tabular}

\section{Appendix 4.9: Interview SME Sizes}

\begin{tabular}{|l|l|l|}
\hline Size & Number & Percentage \\
\hline Micro $(<10)$ & 19 & $63.33 \%$ \\
\hline Small $(<50)$ & 8 & $26.7 \%$ \\
\hline Medium-sized $(<250)$ & 3 & $10 \%$ \\
\hline Total & 30 & $100 \%$ \\
\hline
\end{tabular}


Appendix 4.10: Focus Group Themes and Interview Question Development Map

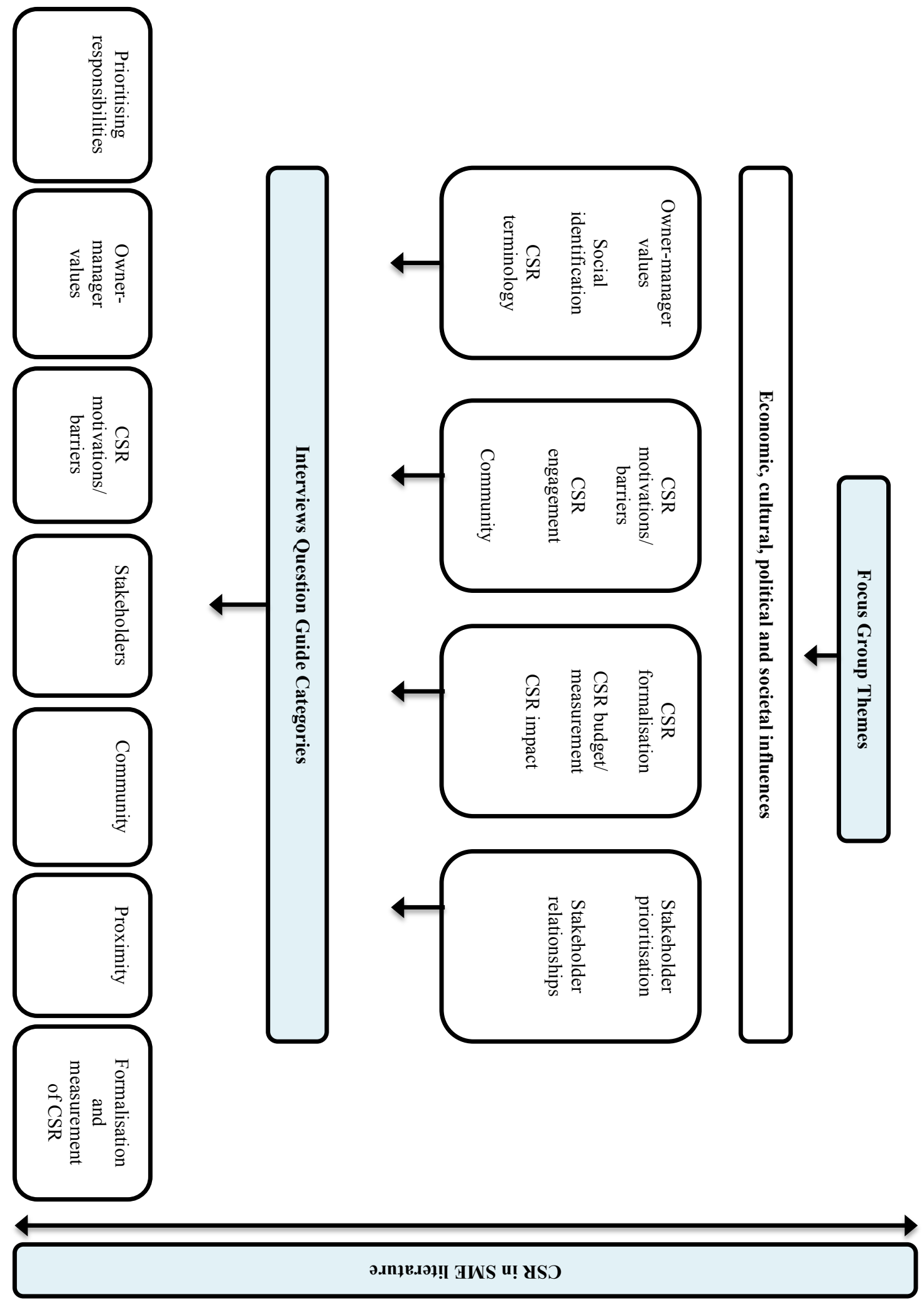




\section{Appendix 4.11: Interview Themes and Questions}

\section{Interview Questions}

Main theme: As a small or medium-sized enterprise (SME), how do you prioritise your responsibilities?

Theme One: $\quad$ Prioritisation of responsibilities

- Do you believe your business makes a contribution to the economy and society?

- In what way does your business make this contribution?

- What would you say are the main responsibilities of your business?

- How do you generally prioritise tasks in your business?

\section{Theme Two: $\quad$ Owner-manager values}

- What are the main values (principles or standards of behaviour) you prioritise as a business owner/manager?

- What are the main responsibilities you prioritise as a business owner/manager?

- Are these values permeated throughout the business?

- What kinds of business decisions are most likely to cause moral consideration for you as owner/manager?

- On what basis do you make a decision in these circumstances?

- Which types of responsibilities do you consider to be most important to yourself and your family as an owner-manager (survival, personal integrity, ethic of care and philanthropic)? Please rank them in order of importance.

Theme Three:

\section{Stakeholders}

- Which stakeholders would you consider to be your main priority?

- On what basis did you prioritise these stakeholders? 
- Do you have one salient stakeholder of your business and what is the balance of control like?

- Could you describe your relationship with your main stakeholders

- What responsibilities do you think you have to your stakeholders?

- Which types of responsibilities do you consider to be most important in terms of your responsibility to your employees (survival, personal integrity, ethic of care and philanthropic)? Please rank them in order of importance.

- Which types of responsibilities do you consider to be most important in terms of your responsibility to your business partners (survival, personal integrity, ethic of care and philanthropic)? Please rank them in order of importance.

(For the purposes of this research, business partners includes suppliers, customers and competitors)

- Which types of responsibilities do you consider to be most important in terms of your responsibility to the local community (survival, personal integrity, ethic of care and philanthropic)? Please rank them in order of importance (derived from...Jamali, Zanhour, Keshishian, 2009)

- How do you deal with these responsibilities to your stakeholders?

- Do you use any particular strategies, actions or decision criteria in order to deal with them in the best way you can?

\section{Theme Four: $\quad$ Proximity}

- How would you define 'local community' in terms of geography or distance?

- Are your main stakeholders within close geographical proximity of your organisation?

- Where do you find most of your employees come from?

- Do you have good relations with your employees?

- Is your family life integrated or completely separate to your role as an owner/manager?

- Are your customers mainly close by?

- Do you have good relations with your competitors?

- Do you know any of your main stakeholders on a personal level? 
- Do you believe that your main stakeholders share similar values as you?

- What is the geographical scope of the firm's activities?

\section{Theme Five: $\quad$ Community engagement}

- How would you say your organisation interacts with the community?

- What kind of activities does your organisation get involved in?

- Does your firm donate money or their time/skills for a particular cause?

- Do you ever get approached for help?

- Who is usually responsible for coming up with the ideas for the activity?

- Who decides whether to actually engage in the activity?

- Are activities usually run on an organisation wide scale or individual effort?

- Do you follow a particular process for such activities?

- Do you think the term corporate social responsibility is suitable to describe the activities we are talking about for your SME?

\section{Theme Six: $\quad$ CSR}

- What do you understand by the term corporate social responsibility (CSR)?

- What does the term CSR mean to you?

- If CSR is not the term used for socially responsible behaviour in your organisation, what do you call it (if anything at all)?

- What does this term incorporate for your company?

- Is your business socially responsible in any other way than the activities described previously?

Theme Seven:

Motivations/barriers of social responsibility

- What motivated your organisation to initiate socially responsible activities?

- Do you consider the social responsibility motivations of your firm to be mostly altruistic or strategic?

- In your opinion, have they been successful? 
- What do you see as the benefits from engaging in social responsibility?

- Are there tangible outcomes for your company from these activities?

- Would you say there are any particular group of stakeholders you seek to influence through these activities?

- Do your stakeholders reciprocate such activities?

- What did you see as the barriers to you getting involved in social responsibility?

- What do you see as the barriers of your continued engagement in socially responsible activities?

- What are the time pressures for owner/managers in SMEs?

\section{Theme Eight:}

\section{Measurement of social responsibility}

- Do you have a broad idea of the monetary value you spend on CSR?

- Do you ever obtain feedback regarding your social performance?

- If so, what are the sources of this feedback?

- Do you ever track the outcomes of your socially responsible activities?

- Are you aware of any regulations or systems of certification for CSR in SME's?

- Do you have any certification for your CSR engagement?

- Would certification for CSR be useful or inhibiting to you as an SME?

*Finish with any final points participant may like to make and thank them for participation* 


\section{Appendix 4.12: Example of Focus Group Raw Analysis: Content Analysis Step}

\section{Three and Four}
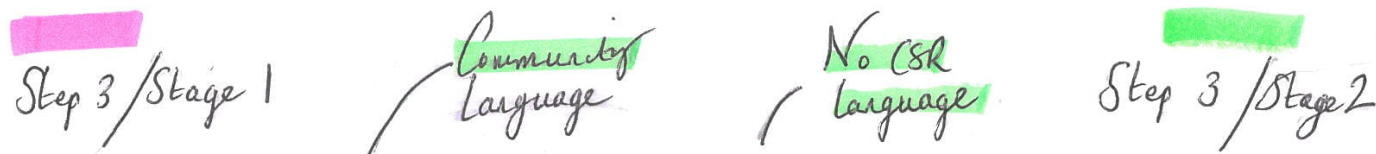

P17: I call it community interaction [cross chat] not corporate social responsibility [cross chat]

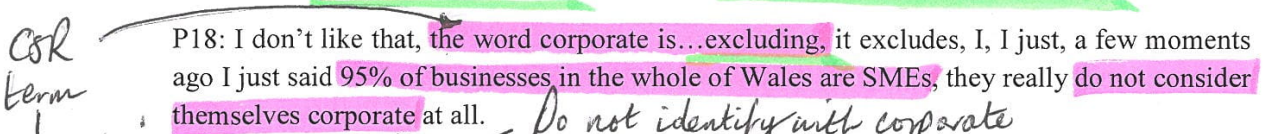

1 themselves corporate at all. Do not identify witt conparate

P20: I think that the other thing with corporate social responsibility implies responsibility for the corporation as a legal entity, but everyone needs a lot more...personal social responsibility as well.

$$
\rightarrow \text { personal social responsibility }
$$

F1: So what language would you use instead of corporate social responsibility?

to P18: I like community. Comm unty as C8R

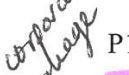

P17: Community is what I think, that's on the records you know, I've always thought that

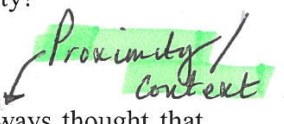
community was social responsibility. Where your business is, where you're doing business.

P20: Or just social responsibility, it doesn't need to be corporate it just needs to have social responsibility [sounds of agreement].

$$
\text { Cexcluding unplied. }
$$

CSR alteruatues

P18: Why don't we just be inclusive of the whole world's situation and just say humanity isn't it because this is, this is about our, our...individual and collective humanity isn't it.

F4: It's a partnership isn't it, because you're relying on businesses but also relying on community as well.

P17: It's whether people buy into it.

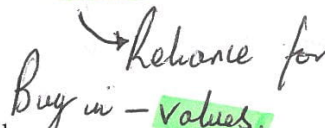

survival

F4: And, and that's a partnership isn't it you know.

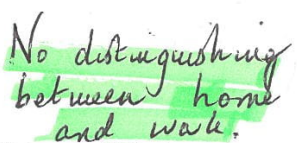

P18: Collaboration.

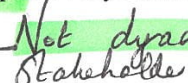

relatworiship?

F4: Yeah collaboration and being respectful so community partners and I don't know if that sums it up but um.

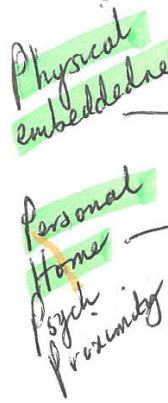

P18: It is an exclusive [/excluding/] word, phrase, it excludes more than it includes doesn't it.

P17: It includes the 'I' you know, the 'me' because nobody cares about 'me'. So you hear the individual cases so how do we get to them, how do we help them meaningfully and sustainably in a business? That's why I call it community, because you are part of the people who are around you and fhat is where your home is so that's why I like community I guess. Tanghbe

P19: I think that it has been labelled as corporate, because that is something tangible for people to see, so you know when you talk about corporate you see the big factories you know you see the output, you see um, you see the big numbers you know and you think more impact but actually, you know, if you've got ninety five per cent of those businesses which are so small, um, y you know versus those big businesses there's more impact you know from the smaller

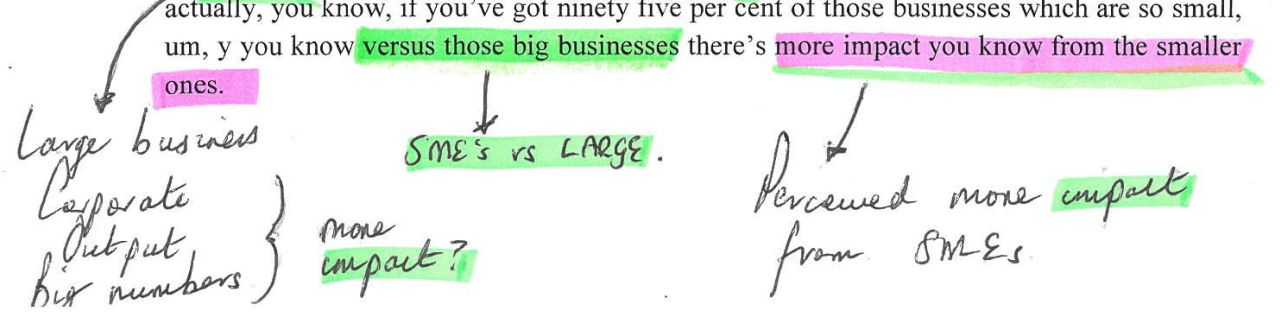




\section{Appendix 4.13: Example of Raw Analysis Interviews: Content Analysis Step Three and Four}

\section{Participant P1:}

$$
\text { Step 3/87age } 1 \quad \text { Step } 3 / 8 \text { tage } 2
$$

I: Do you actually ever see where your donations are going. Do you get any feedback from what you have done or impact? CSR engagenart

P1: Yeah, so we did the you know, wear a hat for brain tumours and stuff like that, and my

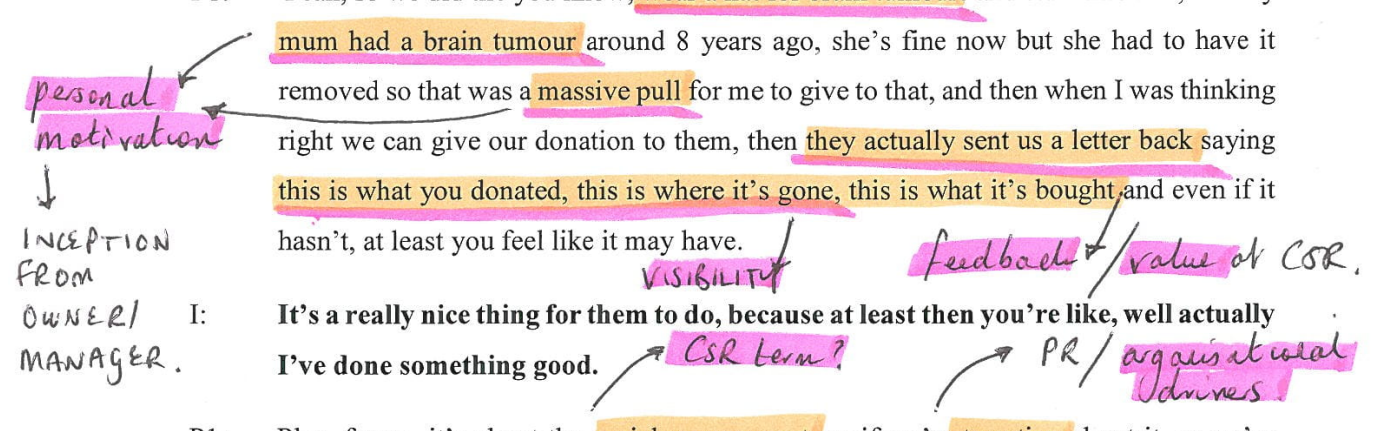

P1: Plus, for us, it's about the social engagement, so if we're tweeting about it, or we're mentioning it, to have them retweet it to all of their followers.

I: Do you use social media a lot?

P1: Yeah, a lot.

I: I guess that's particularly important with your industry, okay so do you know, have you heard of the word corporate social responsibility.

P1: Oh god, no.

I: Okay, so this is my whole point you've done everything that we would describe as corporate social responsibility.

$$
\rightarrow \frac{C O R}{\text { term }}+\text { "corparate" daes not }
$$

P1: It's the corporate, it's like we're not big enough to have to think about it.

I: Yeah, yeah. identificatues nut size

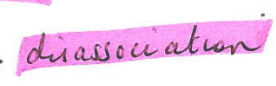

P1: Do you know what I mean? If someone said social responsibility I'd know...

I: But in terms of scale, you're doing a lot towards society and the community compared to some others.

P1: Yeah.

I: So do you understand or have you ever heard of anything to do with CSR? 
P1: $\quad$ No, I don't think so.

I: So in that case, what do you call it?

P1: I'm not really sure, I would say it's a social responsibility, but I don't know..

I: Do you use any other terminology linked to community, community involvement?

P1: Yeah, that, if we were that's what we call it we do tenders for example that's what we call that, it's community involvement definitely

I: Do you do anything linked to sustainability? larguage used by.

P1: Yep, we've got an environmental policy and we've got BS8555 which is a creditation that we've got for, so we have someone come in every year to make sure that we actually are environmentally friendly I guess and that we're actually adhering to that (envisonmental) policy I guess um, so things like, we recycle paper, inks, all the stuff that we use quite $\downarrow \quad$ actually made that a big thing, plus we try and do conference calls as much as we formalisation possibly can to try and save our petrol and diesel and do it in the office to try and get lots of people around a screen rather than carting everyone somewhere

Values?
$\downarrow$ C 8 R engagenent.

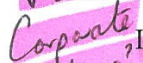

And what motivates you to actually do those socially responsible activities?

P1: Um...it's because we should (laughter) that's the first one, but also when I have

MOTIVATION. Thildren I want them to be able to experience life as I've had it so for things not to be Welf beng disrupted and ruined because of our laziness I suppose, because it is laziness

of futue I: geneations $\downarrow$

Yeah, so I've already asked about reciprocation and you said particularly the University they reciprocate these actions. $\rightarrow$ Relatcoyship/good with

P1: Yeah, I would call it a relationship, I wouldn't say that it was a network, I would say that it was a relationship, if, if I went to anyone in the university and asked them to help, they would help me tomorrow...you know, whereas we don't meet up and network and pass on people's details and stuff like that just because I don't think it's relevant to do that

I: And are there any barriers to being socially responsible? Are there any barriers?

P1: Sometimes money, time, oh my gosh.

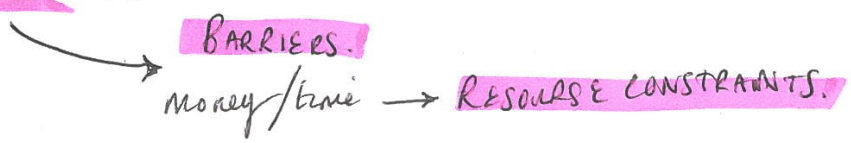




\section{Participant P4:}

Step 3/Stage 1

I: Do you call it anything?

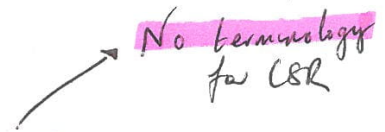

P4: No we don't have a defining name for it, no.

I: But you do understand CSR and what it means?

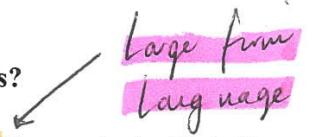

P: Yeah, yeah you know some of the bigger clients I've worked with in the past they've used those terms and they've done particular things that would definitely come under that category. rThey"

P4: Yes, exactly that.

I: Is your business socially responsible in any other way than the activities you've described, such as recycling...? Speciru, CSk pelated to

P4: I guess actually security, there is a social responsibility there that often our products we are using, you know customers are entering their credit card details or whatever and you know there is a responsibility there to that for the public the service you're

I: What motivated you? Do you think that in any cases you get any reciprocation?

VAlues. P4: I do believe in recycling and looking after the planet and I'll do what I can within and what I'm allowed, but it is purely my point of view I suppose. maral deliberatwa betweed

I: Do you think that by being responsible in Sri Lanka, your company gets anything busions from the donations etc? MOTIVATION: ORGANISATIONAL DRIVERS?

P4: It's just brand recognition yeah, so the company over there is associated with doing

Eubeddedres of good, perhaps if some time down the line somebody is applying for a job and they see ralues un

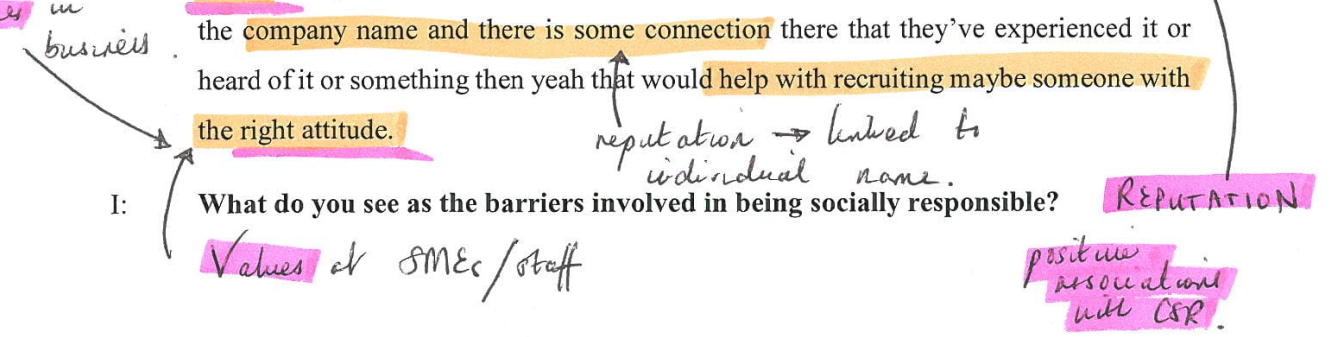




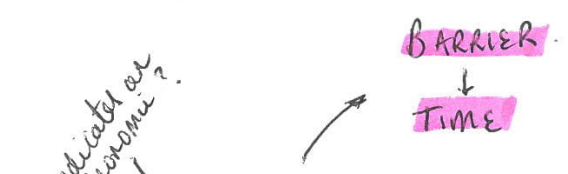

P4: Time is a massive constraint and at the end of the day I've got to make sure there is enough money to run the business and cover my salary so they are always my number one priority. I mean I could give up more of my own time and I think that is probably

CSR - ensuré enough money

to rue business first? what I'm going to have to do to make those connections and make my presence in the community known locally, so I'm just going to have to dofit [laughter].

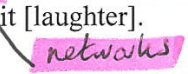

$\triangle$ VISIBILIT.

What do you see as the barriers to continual engagement in these responsibilities?

P4: No, I don't have a problem doing it with my personal skills I think I can talk to most people and happy to get involved. I don't think there are any other barriers involved that I can think of.

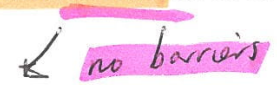

I: Do you have any idea of the amount of money you spend on these activities? $C S R$ engagement

P4: I wouldn't say I'm directly aware of it, I mean I know when I'm discounting rates you Discounts.

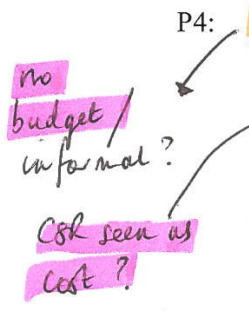
know that is massively eating into the profit margin, so you know I can see that in my reports you know I have quite good monthly financial reports so I can see that if I've charged one rate we would have been a bit more financially better off but you know I don't have an exact figure and I don't worry about it too much, I'm aware of it, but I don't really pay much attention, as long as I'm meeting the other requirements of the business, if it got to the point where it was too much one way then I'd have to rethink business, if it got to the point where it was too much one way then I'd have to rethink
what I'm charging, but at the moment it's a balance yeah. bolave bet ween cor

I: Are we aware of any regulations or systems of certification for social responsibilities in small businesses?

P4: No, not at all. I didn't know there was. No formalisation

I: If there was any certification, do you think it would be useful or inhibiting to small businesses?

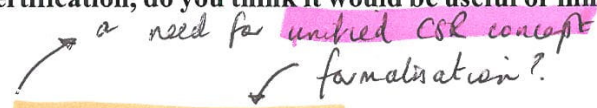

P4: I think that if it was widely known and accepted and that you could sort of badge your

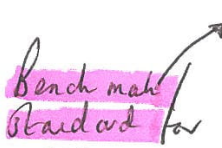
business that you know you are responsible and if everyone was following the same and it was kind of a standardised thing that people recognized and actually went ah I CSR can see they're a good business because they do that and they've got the certification fantastic.

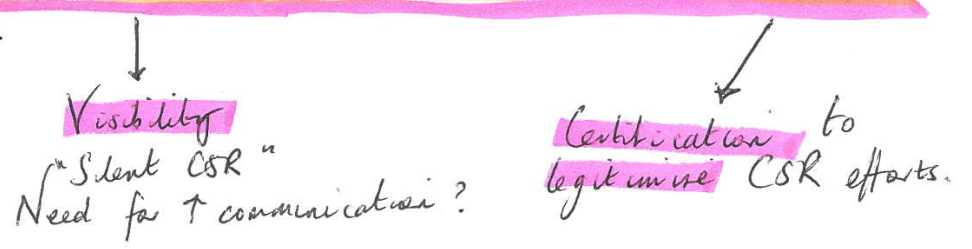




\section{Participant P10:}

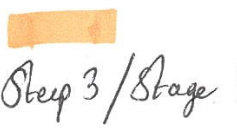

I: So do you believe that your business makes a contribution to the economy and society and in what way?

P10: Well hopefully we do.

I: Obviously.

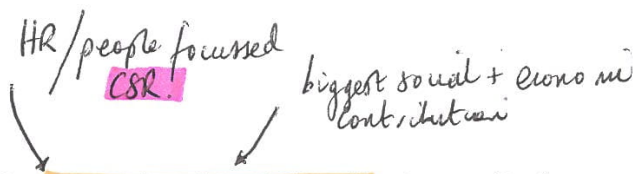

P10: Exactly, yes. Yes I mean now the fact we employ about 105 staff and naturally the older I become I think the more I realise that naturally the business ... the success of

Stof focused the business is so important to keep that number of people employed. You know, the sine. contribution it makes to their families too really. We've got a couple of husband and wife ... well a few husband and wife teams as well and therefore -

I: Oh and their families.

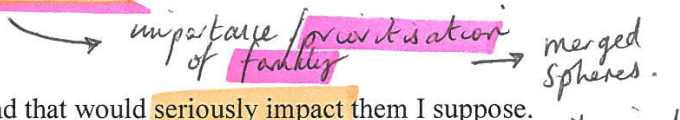

P10: - you know, if we weren't around that would seriously impact them I suppose.

I: What about society?

No P10: Yes, you know we try to operate in a very ethical and responsible way I think and you affir mat we know, we want to be here for the very long-term, as long as we can possibly be, so. I ilains think, you know, we try to preserve our reputation that way and behave in a

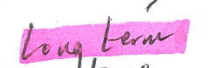

nut we smes. responsible and ethical way and a fair way. We just want to ... we do take it very personally. If we think ... if we've treated people badly we naturally want to put it right and to the best of our ability and we're very happy to put our hands up and acknowledge that things haven't gone too well. I mean hopefully that's not too often, TRANSPARENCY 7 but that's I think hopefully part ... that's part of our ... you know, responsible trading HONESTY pattern as well really.

I: Okay. What would you say are the main responsibilities of your business? Embe dded?

P10: The main responsibilities of our business? Psych promith

I: Yeah it could be anything. It could be like making profit, it could be ... oh, it could be anything. Merged
Spheres
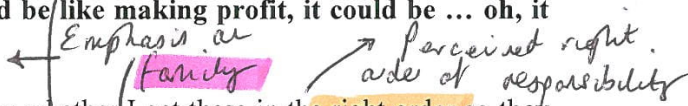

P10: I think it's ... you know, I don't know whether I get these in the right order so they might be sort of ... they might come out urrong, but I think the sort of ... you know, survival really. I mean I'm ... you know, it's a family business, it has been around for 125 years so I'm quite conscious that, you know, I would like it to be around after my days. You know, it's rather nice that, my daughter, has joined the business. She's been here three years. So again I feel we don't ... we haven't got any view to sell it, we would like to be ... you know, just to ... it's very enjoyable running a business so it's ... we want to keep it going. So I really think. Jyou know, survival is the first thing. I think we do try and look at the business in quife a long-term and not short-term.

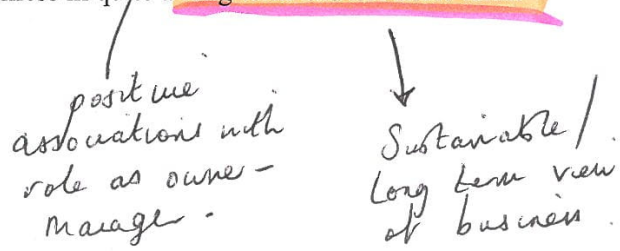




\section{Appendix 4.14: Example of Raw Analysis: Table of Open Codes and Sub-Codes}

\begin{tabular}{|c|c|}
\hline CSR terminology & \\
\hline Open codes & Higher level codes \\
\hline CSR & Derivatives of CSR \\
\hline Social CSR & \\
\hline Social responsibility & \\
\hline Community & Community \\
\hline Community CSR & \\
\hline Local community & \\
\hline Community responsibility & \\
\hline Community benefit & \\
\hline Community interaction & \\
\hline Community partnership & \\
\hline Community collaboration & \\
\hline Partnerships & Partnerships \\
\hline Sustainability & Sustainability \\
\hline Sustainable development & \\
\hline Ethical business & Ethics \\
\hline Humanity & Human values \\
\hline Wellbeing & \\
\hline Love & \\
\hline Happiness & \\
\hline No common language & No common language \\
\hline No name & \\
\hline CSR as not appropriate for SMEs & CSR as excluding \\
\hline 'Corporate' of CSR is excluding for SMEs & \\
\hline
\end{tabular}


Appendix 4.15: Focus Group Coding: Data Visualisation

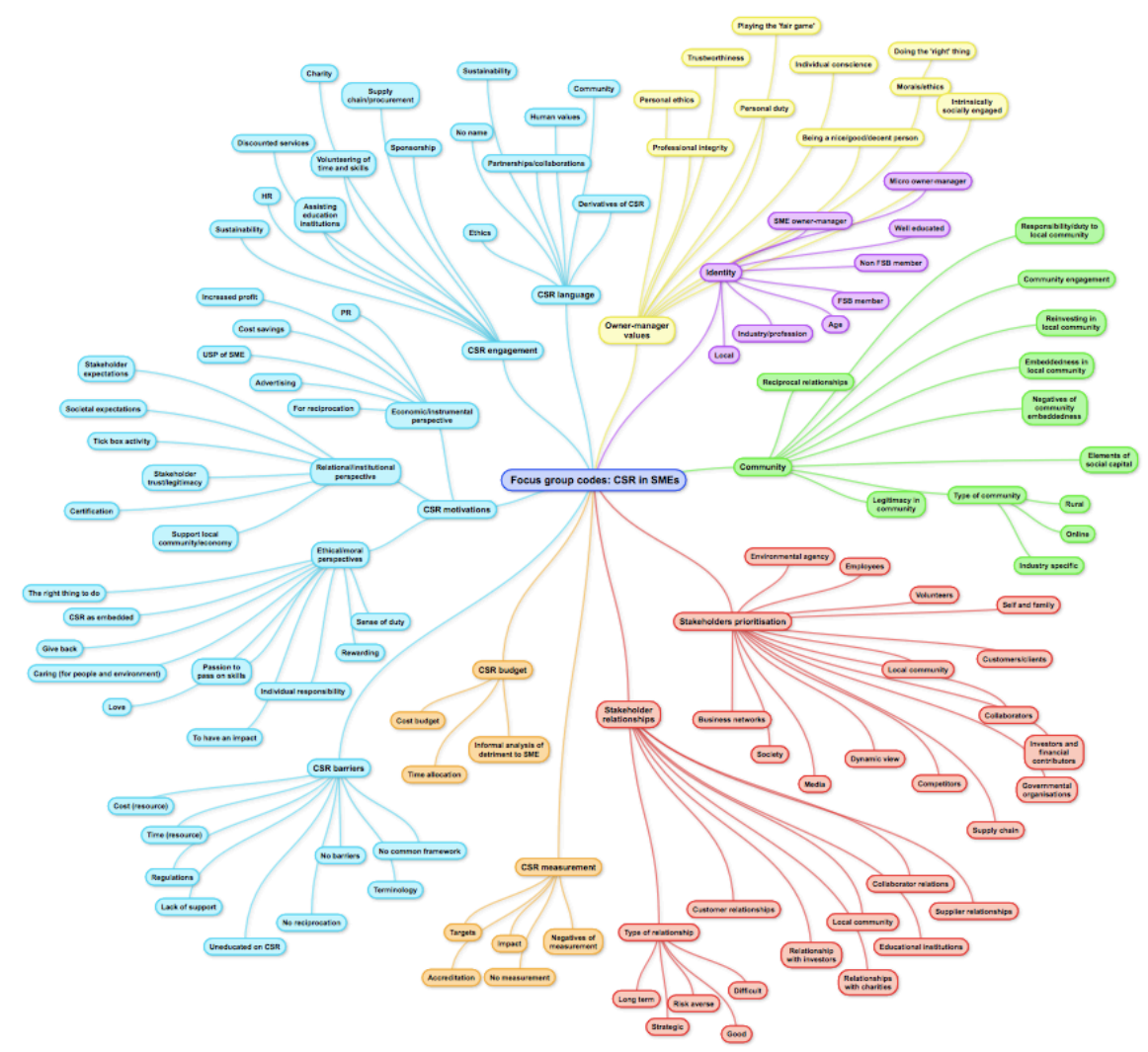


Appendix 5.1: Focus Groups: Summary of Identified Super-Ordinate and SubThemes (derived from open and higher level codes)

Super-Ordinate Themes

CSR Terminology Matters: The Rejection of CSR

Social Identification: "More Socially

Responsible as People"

Owner-Manager Values: An "Embedded Principle"

Drivers and Motivations: "The Right Thing"

Community embeddedness: The "Double Edged Sword"

Stakeholder Relationships: "An Element of Mutual Care"

Stakeholder Prioritisation: "Whoever is Banging at the Table Loudest"

\section{Sub-Themes}

Exclusion and disassociation: "Oh, that's not me then"

CSR language spectrum

Discursive ambiguity

The preservation of identity

The misalignment of large business motives

Ethical superiority

CSR: an embedded principle

Business as an extension of self

Personal social responsibility

Community connections

Power in your pocket

"The right thing"

Giving back

Self-fulfilment

Moral obligation

Addressing a need

Adhering to expectations

Reputation and legitimacy

Economic drivers

Community embeddedness

Reciprocal relationships

Reputation and legitimisation

SME contribution and value

Positive characteristics of closeness

Negative characteristics of closeness

An element of care

Personal connections

Long-term relationships

Stakeholder networks

Synergistic benefits

Friends and family

Staff: the people within

Customers

Community

Contextual

Dynamic 


\begin{tabular}{ll}
\hline & Moral deliberations \\
& Resource constraints \\
"TIME" & Loss of key CSR contact \\
Barriers and Challenges: "A Big Change of & Escalation of requests \\
Mindset" & Increasing demands \\
& Unfair and challenging legislation \\
& Numbers and targets \\
& \\
"Blurred Boundaries" & Political support: positive associations \\
& Political support: negative associations \\
& Inhibiting economic conditions \\
& CSR as a solution \\
& Educating and accommodating \\
& \\
CSR Awareness: Disseminate and Educate & Dissemination of knowledge \\
& SMEs: a vital resource \\
& Silent CSR \\
& Lack of recognition \\
& \\
& Sustainable CSR \\
& Long term value \\
& The wellbeing of future generations \\
\hline & \\
& \\
& \\
& \\
&
\end{tabular}


Appendix 6.1: Interviews: Summary of Identified Super-Ordinate and SubThemes (informed by focus groups, theory and open and higher level codes from interviews)

\begin{tabular}{|c|c|c|}
\hline Super-Ordinate Themes & Sub-Themes & $\begin{array}{l}\text { To establish the relevance } \\
\text { of: }\end{array}$ \\
\hline $\begin{array}{l}\text { The Role of the Owner- } \\
\text { Manager }\end{array}$ & $\begin{array}{l}\text { Owner-manager morals, } \\
\text { characteristics and values }\end{array}$ & $\begin{array}{c}\text { Spence's (2016) SBSR } \\
\text { Pyramid }\end{array}$ \\
\hline \multirow[t]{2}{*}{ Cognitive Awareness of CSR } & CSR terminology & $\begin{array}{c}\text { Spence's (2016) SBSR } \\
\text { Pyramid }\end{array}$ \\
\hline & CSR and conceptual nuances & \\
\hline \multirow[t]{3}{*}{$\begin{array}{l}\text { Individual Conceptualisation } \\
\text { of SME Responsibilities }\end{array}$} & $\begin{array}{l}\text { Survival, economic } \\
\text { contributions and sustainable } \\
\text { business }\end{array}$ & $\begin{array}{c}\text { Spence's (2016) SBSR } \\
\text { Pyramid }\end{array}$ \\
\hline & $\begin{array}{l}\text { Legal compliance } \\
\text { Ethical standards, norms and } \\
\text { expectations }\end{array}$ & \\
\hline & Philanthropic responsibilities & \\
\hline \multirow[t]{3}{*}{$\begin{array}{l}\text { Motivations and Drivers of } \\
\text { CSR }\end{array}$} & $\begin{array}{l}\text { Intrinsic motivations and moral } \\
\text { obligations }\end{array}$ & $\begin{array}{c}\text { Spence's (2016) SBSR } \\
\text { Pyramid }\end{array}$ \\
\hline & $\begin{array}{l}\text { Organisational motives and } \\
\text { relational drivers }\end{array}$ & \\
\hline & $\begin{array}{l}\text { Instrumental drivers and } \\
\text { tangible outcomes }\end{array}$ & \\
\hline \multirow[t]{6}{*}{$\begin{array}{l}\text { Characterisation of } \\
\text { Stakeholder Relationships }\end{array}$} & $\begin{array}{l}\text { Family, friendship and long- } \\
\text { term commitment }\end{array}$ & $\begin{array}{c}\text { Spence's (2016) redrawn } \\
\text { Stakeholder theory }\end{array}$ \\
\hline & $\begin{array}{l}\text { Inextricable personal and } \\
\text { business connections }\end{array}$ & \\
\hline & $\begin{array}{l}\text { Community embeddedness, } \\
\text { reputation and word of mouth }\end{array}$ & \\
\hline & Networks and collaborations & \\
\hline & The dynamic nature of SMEs & \\
\hline & $\begin{array}{l}\text { Stakeholder relationships and } \\
\text { the balance of power }\end{array}$ & \\
\hline
\end{tabular}


Appendix 8.1: System Orientated View of SME Stakeholder Theory

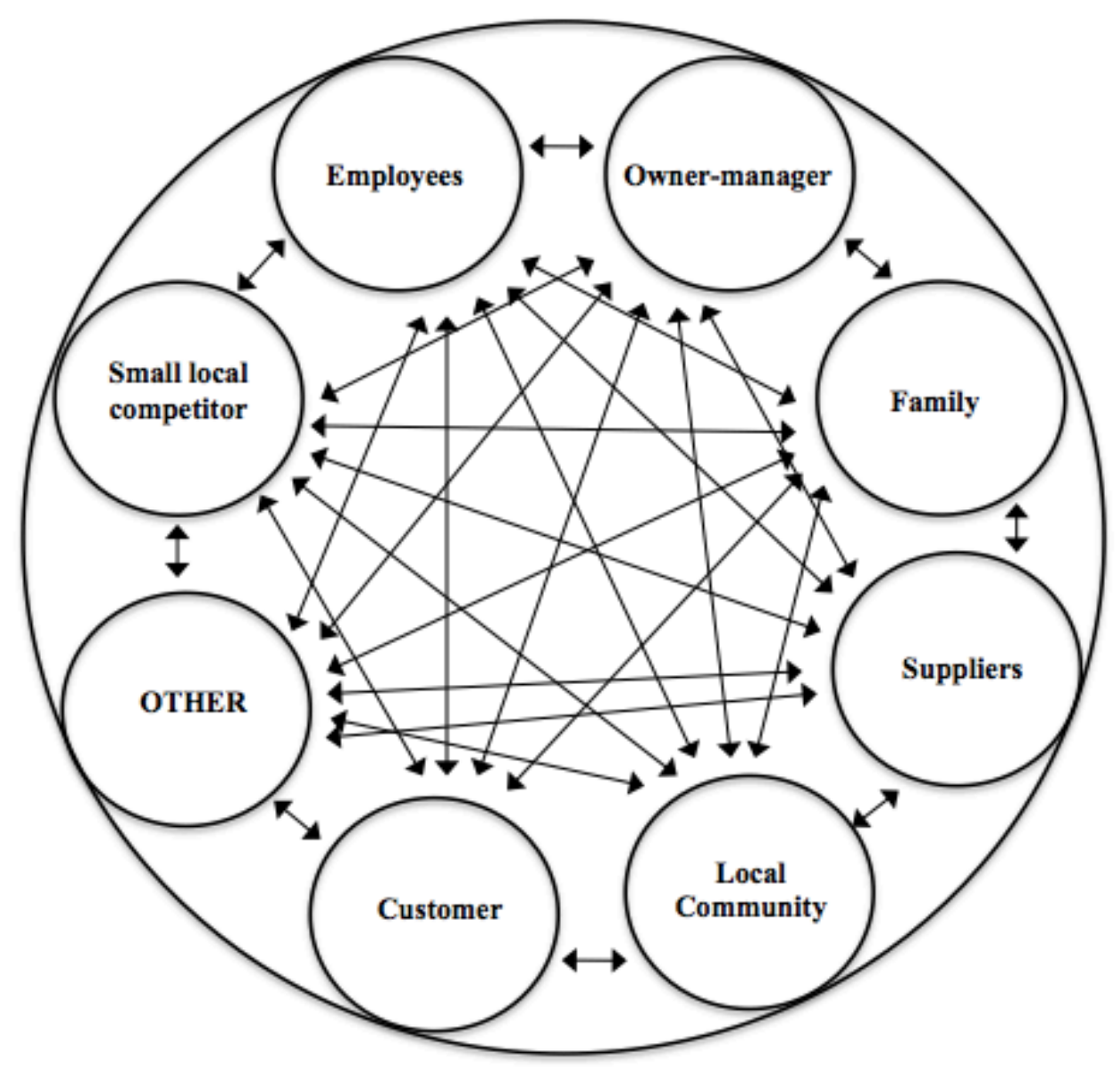

